\title{
Supporting Information \\ Rhodium-Catalyzed Synthesis of Amides from Functionalized Blocked Isocyanates
}

\author{
Joshua S. Derasp, André M. Beauchemin* \\ Centre for Catalysis Research and Innovation, Department of Chemistry and Biomolecular Sciences \\ University of Ottawa, Ottawa, Ontario, Canada, K1N 6N5 \\ *andre.beauchemin@uottawa.ca
}

\section{Supporting Information}

\section{Contents}

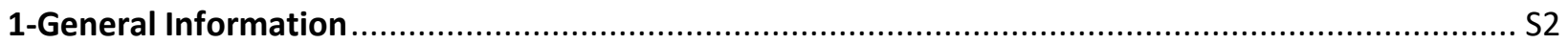

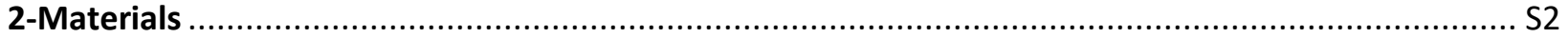

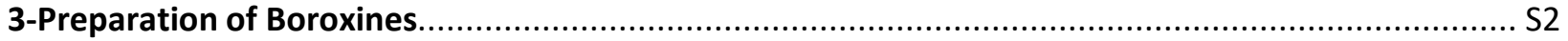

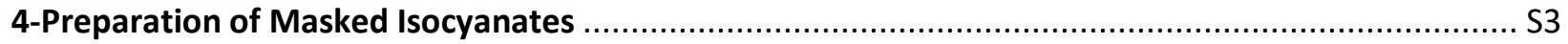

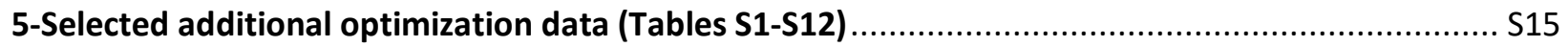

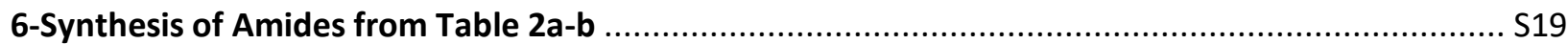

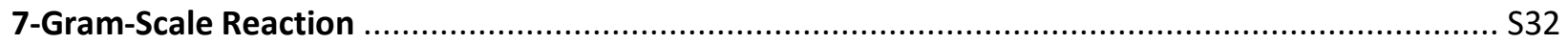

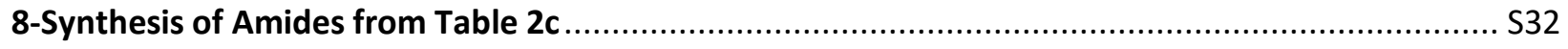

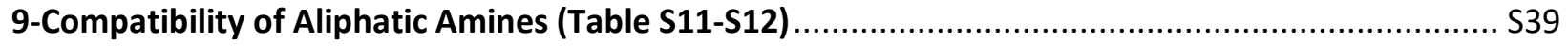

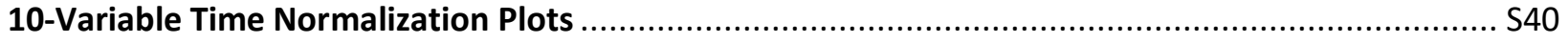

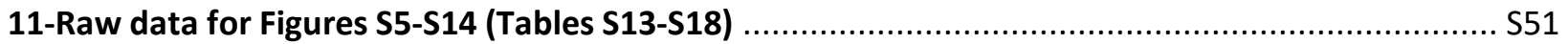

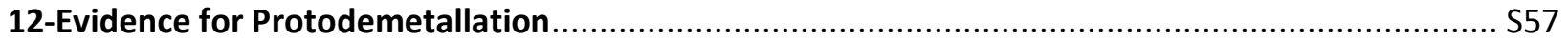

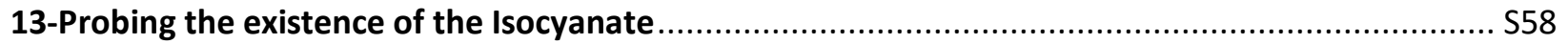

14-Probing reversibility - formation of tryptophan derived dimer (3vva) ...................................... S58

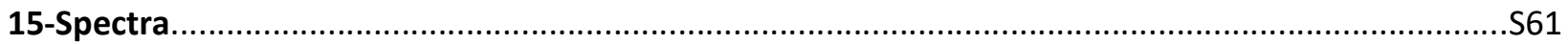




\section{1-General Information}

Purification of reaction products was carried out by flash column chromatography using SiliCycle silica gel (40-63 $\mu \mathrm{m})$, unless otherwise noted. Analytical thin layer chromatography (TLC) was performed on aluminum, cut to size. Visualization was accomplished with UV light followed by staining with a potassium permanganate solution and heating.

${ }^{1} \mathrm{H}$ and ${ }^{13} \mathrm{C}$ NMR spectra were recorded on Bruker AVANCE $300 \mathrm{MHz}$ and $400 \mathrm{MHz}$ spectrometers at ambient temperature, unless otherwise indicated. Spectral data was reported in ppm using solvent as the reference $\left(\mathrm{CDCl}_{3}\right.$ at $7.26 \mathrm{ppm}$ or DMSO- $d_{6}$ at $2.50 \mathrm{ppm}$ for ${ }^{1} \mathrm{H} \mathrm{NMR}$ and $\mathrm{CDCl}_{3}$ at $77.0 \mathrm{ppm}$ or DMSO- $d_{6}$ at 39.43 for ${ }^{13} \mathrm{C}$ NMR). ${ }^{1} \mathrm{H}$ NMR data was reported as: multiplicity (br = broad, $\mathrm{s}=$ singlet, $\mathrm{d}$ = doublet, $\mathrm{t}$ = triplet, $\mathrm{q}$ = quartet, quint. = quintet, sext. = sextuplet, sept = septuplet, $\mathrm{m}$ = multiplet), integration and coupling constant(s) in Hz. Carbon resonances were assigned using dept 135 spectra. Infrared (IR) spectra were obtained on neat samples using an Attenuated Total Reflectance Fourier transform infrared spectrometer (ATR-FTIR). High-resolution mass spectroscopy (HRMS) was performed on a Kratos Concept-11A mass spectrometer with an electron beam of $70 \mathrm{eV}$ (EI) or Micromass Q-TOF I- Time of Flight Electrospray Ionization mass spectrometer (ESI) at the OttawaCarleton Mass Spectrometry Centre. Enantiomeric excess was determined using Agilent technologies 1200 series HPLC. Optical rotations were measured using Anton Paar mcp500 polarimeter.

\section{2-Materials}

Unless otherwise noted, all commercially available materials were purchased from commercial sources and used without further purification.

\section{3-Preparation of Boroxines}

-Arylboroxines were readily prepared using literature procedures. ${ }^{1}$ Arylboronic acids were heated to 110 ${ }^{\circ} \mathrm{C}$ overnight under reduced pressure and the corresponding boroxines were stored in a desiccator. Recrystallization of the boronic acid in water before dehydration resulted in a profound increase in reaction efficiency in some cases (3ag). This allowed the catalyst loading to diminish from $2.5 \mathrm{~mol} \%[\mathrm{Rh}]_{2}$ before without prior recrystallization (61\% yield) to $0.5 \mathrm{~mol} \%[\mathrm{Rh}]_{2}$ ( $71 \%$ yield). The generality of this observation was not established. 


\section{4-Preparation of Masked Isocyanates}

General procedure A: $\mathrm{NaHCO}_{3}(3.30 \mathrm{mmol}, 1.10$ equiv.) was added to a round bottom flask containing THF:water (10.0 mL, 3:1) followed by the addition of the amine ( $3.00 \mathrm{mmol}, 1.00$ equiv.). The temperature of the solution was brought to $0{ }^{\circ} \mathrm{C}$ upon which the phenyl chloroformate $(3.15 \mathrm{mmol}, 1.05$ equiv.) was added dropwise. The solution was allowed to warm to room temperature and subsequently monitored for the disappearance of starting material (generally 0.5 to 2 hours). Upon completion, the reaction was diluted with EtOAc $(40 \mathrm{~mL})$, then extracted with water $(1 \times 30 \mathrm{~mL})$ and brine $(1 \times 30 \mathrm{~mL})$. The organic layer was collected, dried over $\mathrm{Na}_{2} \mathrm{SO}_{4}$, and concentrated under reduced pressure. The crude extract was purified by silica gel chromatography to give the corresponding products.

General procedure B: An amine (3.00 mmol, 1.00 equiv.), $\mathrm{CH}_{2} \mathrm{Cl}_{2}(0.3 \mathrm{M})$, and $\mathrm{Et}_{3} \mathrm{~N}$ (3.30 mmol, 1.10 equiv.) were added to a dry round bottom flask. The temperature of the solution was brought to $0{ }^{\circ} \mathrm{C}$ upon which phenyl chloroformate $(3.15 \mathrm{mmol}, 1.05$ equiv.) was added dropwise. The solution was warmed to room temperature and subsequently monitored for the disappearance of starting material. The reaction was concentrated under reduced pressure, resuspended in EtOAc $(40 \mathrm{~mL})$ and extracted with water $(1 \times 30 \mathrm{~mL})$ and brine $(1 \times 30 \mathrm{~mL})$. The organic layer was collected, dried over sodium sulfate, and concentrated under reduced pressure. The crude extract was purified by silica gel chromatography to give the corresponding products.

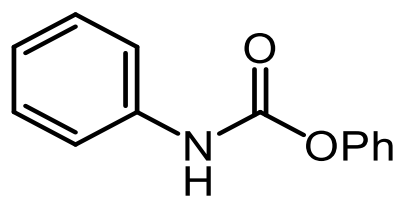

phenyl phenylcarbamate (1a): Synthesized according to general procedure A using aniline $(0.913 \mathrm{~mL}$, $10.0 \mathrm{mmol})$, phenyl chloroformate $(1.32 \mathrm{~mL}, 10.5 \mathrm{mmol})$, and $\mathrm{NaHCO}_{3}(0.924 \mathrm{~g}, 11.0 \mathrm{mmol})$. The reaction reached completion within 0.5 hours (note: Degradation to urea byproduct detected when left for 2 hours). The desired product was extracted pure from the reaction mixture yielding an amorphous white solid (1.91 g, 90\% yield). TLC $\mathrm{R}_{\mathrm{F}}=0.53$ in $60 \% \mathrm{CH}_{2} \mathrm{Cl}_{2} /$ hexanes. ${ }^{1} \mathrm{H}-\mathrm{NMR}$ (DMSO- $\left.d_{6}\right) \delta 7.01(1 \mathrm{H}, \mathrm{t}$, $J=7.4 \mathrm{~Hz}), 7.17-7.24(3 \mathrm{H}, \mathrm{m}), 7.29(2 \mathrm{H}, \mathrm{t}, J=8.0 \mathrm{~Hz}), 7.39(2 \mathrm{H}, \mathrm{t}, J=5.3 \mathrm{~Hz}), 7.48(1 \mathrm{H}, \mathrm{d}, J=7.7 \mathrm{~Hz}), 10.19(1 \mathrm{H}$, s). ${ }^{13} \mathrm{C}-\mathrm{NMR}\left(100 \mathrm{MHz}, \mathrm{CDCl}_{3}\right): \delta 118.9(\mathrm{CH}), 121.8(\mathrm{CH}), 123.9(\mathrm{CH}), 125.8(\mathrm{CH}), 129.2(\mathrm{CH}), 129.5(\mathrm{CH})$, 137.5 (C), 150.6 (C), 151.9 (C). Data is consistent with literature. ${ }^{2}$ 
<smiles>COc1ccc(NC(=O)c2ccccc2)cc1OC</smiles>

phenyl (3-phenyl (3,4-dimethoxyphenyl)carbamate (1b): Synthesized according to general procedure $\mathbf{A}$ using 3,4-dimethoxyaniline $(0.766 \mathrm{~g}, 5.00 \mathrm{mmol})$, phenyl chloroformate $(0.569 \mathrm{~mL}, 5.25 \mathrm{mmol})$, and $\mathrm{NaHCO}_{3}(0.462 \mathrm{~g}, 5.50 \mathrm{mmol})$. The reaction reached completion within 0.5 hours. The desired product was extracted pure from the reaction mixture yielding an amorphous purple/brown solid $(1.17 \mathrm{~g}, 86 \%$ yield). TLC $\mathrm{RF}_{\mathrm{F}}=0.26$ in $\mathrm{CH}_{2} \mathrm{Cl}_{2} .{ }^{1} \mathrm{H}-\mathrm{NMR}\left(300 \mathrm{MHz}, \mathrm{CDCl}_{3}\right): \delta 3.82(6 \mathrm{H}, \mathrm{s}), 6.78(2 \mathrm{H}, \mathrm{m}), 7.02(1 \mathrm{H}, \mathrm{s}), 7.14-$ $7.25(4 \mathrm{H}, \mathrm{m}), 7.36(2 \mathrm{H}, \mathrm{t}, \mathrm{J}=6.9 \mathrm{~Hz}) .{ }^{13} \mathrm{C}-\mathrm{NMR}\left(75 \mathrm{MHz}\right.$, Acetone- $\left.d_{6}\right): \delta 55.1\left(\mathrm{CH}_{3}\right), 55.6\left(\mathrm{CH}_{3}\right), 104.2(\mathrm{CH})$, $110.6(\mathrm{CH}), 112.6(\mathrm{CH}), 121.8(\mathrm{CH}), 125.1(\mathrm{CH}), 129.1(\mathrm{CH}), 132.4(\mathrm{C}), 145.7(\mathrm{C}), 149.7(\mathrm{C}), 151.2(\mathrm{C}), 151.8$ (C). IR (film): 3339, 1741, $1191 \mathrm{~cm}^{-1}$. HRMS (EI): Exact mass calcd for $\mathrm{C}_{15} \mathrm{H}_{15} \mathrm{NO}_{4}$ [M] ${ }^{+}: 273.1001$. Found: 273.1011.<smiles>COC(=O)c1cc(NC(=O)Oc2ccccc2)cc(C(=O)OC)c1</smiles>

dimethyl 5-((phenoxycarbonyl)amino)isophthalate (1c): Synthesized according to general procedure A using dimethyl 5 -aminoisophthalate $(0.628 \mathrm{~g}, 3.00 \mathrm{mmol})$, phenyl chloroformate $(0.395 \mathrm{~mL}, 3.15 \mathrm{mmol})$, and $\mathrm{NaHCO}_{3}(0.277 \mathrm{~g}, 0.330 \mathrm{mmol})$. The reaction reached completion within 2 hours. The desired product was dry loaded onto silica gel $(\mathrm{MeOH})$ and purified by column chromatography $(80 \%$ $\mathrm{CH}_{2} \mathrm{Cl}_{2}$ /hexanes) yielding an amorphous white solid ( $0.750 \mathrm{~g}$, 76\% yield). TLC $\mathrm{R}_{\mathrm{F}}=0.24$ in $80 \%$ $\mathrm{CH}_{2} \mathrm{Cl}_{2}$ /hexanes. ${ }^{1} \mathrm{H}-\mathrm{NMR}\left(300 \mathrm{MHz}\right.$, DMSO- $\left.d_{6}\right): \delta 3.82(6 \mathrm{H}, \mathrm{s}), 7.21-7.25(3 \mathrm{H}, \mathrm{m}), 7.40(2 \mathrm{H}, \mathrm{t}, \mathrm{J}=7.9 \mathrm{~Hz})$, $8.01(1 \mathrm{H}, \mathrm{s}), 8.29(2 \mathrm{H}, \mathrm{s}), 10.58(1 \mathrm{H}, \mathrm{s}) .{ }^{13} \mathrm{C} \mathrm{NMR}\left(100 \mathrm{MHz}, \mathrm{DMSO}-d_{6}\right): 52.8\left(\mathrm{CH}_{3}\right), 122.3(\mathrm{CH}), 123.1(\mathrm{CH})$, 124.2 (CH), $126.1(\mathrm{CH}), 129.9(\mathrm{CH}), 131.1$ (C), 140.1 (C), 150.8 (C), 152.1 (C), 165.6 (C). IR (film): 3268, 1702, $1217 \mathrm{~cm}^{-1}$. HRMS (EI): Exact mass calcd for $\mathrm{C}_{17} \mathrm{H}_{15} \mathrm{NO}_{6}[\mathrm{M}]^{+}: 329.0899$. Found: 329.0916. 
<smiles>CC(=O)c1cccc(NC(=O)Oc2ccccc2)c1</smiles>

phenyl (3-acetylphenyl)carbamate (1d): Synthesized according to general procedure A using 1-(3aminophenyl)ethanone $(0.676 \mathrm{~g}, 5.00 \mathrm{mmol})$, phenyl chloroformate $(0.569 \mathrm{~mL}, 5.25 \mathrm{mmol})$, and $\mathrm{NaHCO}_{3}$ $(0.462 \mathrm{~g}, 5.50 \mathrm{mmol})$. The reaction reached completion within 0.5 hours. The desired product was extracted pure from the reaction mixture yielding an amorphous white solid $\left(0.948 \mathrm{~g}, 74 \%\right.$ yield). TLC $\mathrm{R}_{\mathrm{F}}$ $=0.18$ in $5 \% \mathrm{CH}_{2} \mathrm{Cl}_{2} /$ EtOAc. ${ }^{1} \mathrm{H}-\mathrm{NMR}\left(400 \mathrm{MHz}, \mathrm{CDCl}_{3}\right): \delta 2.59(3 \mathrm{H}, \mathrm{s}), 7.12-7.19(3 \mathrm{H}, \mathrm{m}), 7.24(1 \mathrm{H}, \mathrm{m})$, 7.37-7.44 (3H, m), 7.66-7.73 (2H, m), 8.03 (1H, s). ${ }^{13} \mathrm{C}-\mathrm{NMR}\left(100 \mathrm{MHz}, \mathrm{CDCl}_{3}\right): \delta 26.7\left(\mathrm{CH}_{3}\right), 118.6(\mathrm{CH})$, $121.7(\mathrm{CH}), 123.4(\mathrm{CH}), 123.7(\mathrm{CH}), 125.9(\mathrm{CH}), 129.4(\mathrm{CH}), 129.5(\mathrm{CH}), 137.8(\mathrm{C}), 138.2(\mathrm{C}), 150.4(\mathrm{C})$, 152.0 (C), 198.3 (C). IR (film): $3311,1735,1670,1189 \mathrm{~cm}^{-1}$. HRMS (EI): Exact mass calcd for $\mathrm{C}_{15} \mathrm{H}_{13} \mathrm{NO}_{3}$ $[\mathrm{M}]^{+}:$255.0895. Found: 255.0873.<smiles>O=C(Nc1cccc(Cl)c1)Oc1ccccc1</smiles>

phenyl (3-chlorophenyl)carbamate (1e): Synthesized according to general procedure $\mathbf{A}$ using 3chloroaniline $(0.317 \mathrm{~mL}, 3.00 \mathrm{mmol})$, phenyl chloroformate $(0.395 \mathrm{~mL}, 3.15 \mathrm{mmol})$, and $\mathrm{NaHCO}_{3}(0.277$ $\mathrm{g}, 0.330 \mathrm{mmol}$ ). The reaction reached completion within 2 hours. The desired product was dry loaded onto silica gel ( $\mathrm{MeOH})$ and purified by column chromatography $\left(50 \% \mathrm{CH}_{2} \mathrm{Cl}_{2} /\right.$ hexanes) yielding an amorphous white solid $\left(0.533 \mathrm{~g}, 72 \%\right.$ yield) TLC $\mathrm{R}_{\mathrm{F}}=0.45$ in $50 \% \mathrm{CH}_{2} \mathrm{Cl}_{2} /$ hexanes. ${ }^{1} \mathrm{H}-\mathrm{NMR}(400 \mathrm{MHz}$, $\left.\mathrm{CDCl}_{3}\right): \delta 7.05(1 \mathrm{H}, \mathrm{d}, J=7.1 \mathrm{~Hz}), 7.14-7.26(6 \mathrm{H}, \mathrm{m}), 7.37(2 \mathrm{H}, \mathrm{t}, J=8.4 \mathrm{~Hz}), 7.49(1 \mathrm{H}, \mathrm{s}) .{ }^{13} \mathrm{C}-\mathrm{NMR}(100 \mathrm{MHz}$, $\left.\mathrm{CDCl}_{3}\right): \delta 117.0(\mathrm{CH}), 119.0(\mathrm{CH}), 121.7(\mathrm{CH}), 124.0(\mathrm{CH}), 126.0(\mathrm{CH}), 129.5(\mathrm{CH}), 130.1(\mathrm{CH}), 134.8(\mathrm{C})$, 138.7 (C), 150.4 (C), 151.8 (C). IR (film): 3389, 1758, 1192, $784 \mathrm{~cm}^{-1}$. HRMS (EI): Exact mass calcd for $\mathrm{C}_{13} \mathrm{H}_{10} \mathrm{ClNO}_{2}[\mathrm{M}]^{+}: 247.0400$. Found: 247.0406.<smiles>O=C(Nc1ccc(Br)cc1)Oc1ccccc1</smiles>

phenyl (4-bromophenyl)carbamate (1f): Synthesized according to general procedure $\mathbf{A}$ using 4bromoaniline $(0.516 \mathrm{~g}, 3.00 \mathrm{mmol})$, phenyl chloroformate $(0.395 \mathrm{~mL}, 3.15 \mathrm{mmol})$, and $\mathrm{NaHCO}_{3}(0.277 \mathrm{~g}$, 
$0.330 \mathrm{mmol}$ ). The reaction reached completion within 2 hours. The desired product was dry loaded onto silica gel $(\mathrm{MeOH})$ and purified by column chromatography $\left(50 \% \mathrm{CH}_{2} \mathrm{Cl}_{2} /\right.$ hexanes) yielding an amorphous white solid $\left(0.707 \mathrm{~g}\right.$, 81\% yield). $\mathrm{TLC} \mathrm{R} F=0.45$ in $50 \% \mathrm{CH}_{2} \mathrm{Cl}_{2} /$ hexanes. ${ }^{1} \mathrm{H}-\mathrm{NMR}\left(400 \mathrm{MHz}, \mathrm{CDCl}_{3}\right): \delta 7.12-$ 7.16 $(3 \mathrm{H}, \mathrm{m}), 7.21-7.30(3 \mathrm{H}, \mathrm{m}), 7.35-7.41(6 \mathrm{H}, \mathrm{m}) .{ }^{13} \mathrm{C}-\mathrm{NMR}\left(100 \mathrm{MHz}, \mathrm{CDCl}_{3}\right): \delta 116.5(\mathrm{C}), 120.4(\mathrm{CH})$, $121.7(\mathrm{CH}), 125.9(\mathrm{CH}), 129.5(\mathrm{CH}), 132.1(\mathrm{CH}), 136.5(\mathrm{C}), 150.4(\mathrm{C}), 151.7$ (C). IR (film): 3304, 1711, 1218, $687 \mathrm{~cm}^{-1}$. HRMS (EI): Exact mass calcd for $\mathrm{C}_{13} \mathrm{H}_{10} \mathrm{NO}_{2} \mathrm{Br}[\mathrm{M}]^{+}: 290.9895$. Found: 290.9895.<smiles>Cc1cc(C)c(NC(=O)c2ccccc2)c(C)c1</smiles>

phenyl mesitylcarbamate (19): Synthesized according to general procedure B using 2,4,6trimethylaniline $(0.421 \mathrm{~mL}, 3.00 \mathrm{mmol})$, phenyl chloroformate $(0.395 \mathrm{~mL}, 3.15 \mathrm{mmol})$, and $\mathrm{Et}_{3} \mathrm{~N}(0.460$ $\mathrm{mL}, 3.30 \mathrm{mmol}$ ). The reaction reached completion within $16 \mathrm{~h}$. The crude mixture was purified using column chromatography ( $80 \% \mathrm{CH}_{2} \mathrm{Cl}_{2}$ /hexanes) providing the pure product as an amorphous white solid ( $0.688 \mathrm{~g}, 90 \%$ yield). TLC RF $=0.36$ in $80 \% \mathrm{CH}_{2} \mathrm{Cl}_{2} /$ hexanes ${ }^{1} \mathrm{H}-\mathrm{NMR}\left(400 \mathrm{MHz}, \mathrm{CDCl}_{3}\right): \delta 2.30(7.1 \mathrm{H}, \mathrm{s})$, $2.37(1.9 \mathrm{H}, \mathrm{s}), 6.48(1 \mathrm{H}, \mathrm{s}, \mathrm{br}), 6.92-6.96(2 \mathrm{H}, \mathrm{m}, \mathrm{br}), 7.09(0.6 \mathrm{H}, \mathrm{m}, \mathrm{br}), 7.20-7.24(2.4 \mathrm{H}, \mathrm{m}, \mathrm{br}), 7.32-7.41$ $(2 \mathrm{H}, \mathrm{m})$ (note: rotamers present). ${ }^{13} \mathrm{C}-\mathrm{NMR}(100 \mathrm{MHz}, \mathrm{CDCl}): \delta 18.4\left(\mathrm{CH}_{3}\right), 21.0\left(\mathrm{CH}_{3}\right), 121.5(\mathrm{CH}), 121.7$ $(\mathrm{CH}), 125.3(\mathrm{CH}), 125.5(\mathrm{CH}), 129.1(\mathrm{CH}), 129.4(\mathrm{CH}), 130.6(\mathrm{C}), 131.4(\mathrm{CH}), 135.6(\mathrm{C}), 135.9(\mathrm{C}), 137.4(\mathrm{C})$, 137.1 (C), 151.0 (C), 151.2 (C), 152.7 (C), 154.6 (C) (note: rotamers present). IR (film): 3275, 1704, 1238 $\mathrm{cm}^{-1}$. HRMS (EI): Exact mass calcd for $\mathrm{C}_{16} \mathrm{H}_{17} \mathrm{NO}_{2}[\mathrm{M}]^{+}:$: 255.1259. Found: 255.1273.<smiles>O=C(Nc1c([In])cccc1[Te])Oc1ccccc1</smiles>

phenyl (2,6-diisopropylphenyl)carbamate (1h): Synthesized according to general procedure B using 2,6diisopropylaniline $(0.566 \mathrm{~mL}, 3.00 \mathrm{mmol})$, phenyl chloroformate $(0.395 \mathrm{~mL}, 3.15 \mathrm{mmol})$, and $\mathrm{Et}_{3} \mathrm{~N}(0.459$ $\mathrm{mL}, 3.30 \mathrm{mmol})$. The reaction time was for $16 \mathrm{~h}$. The crude mixture was purified using column chromatography $\left(50 \% \mathrm{CH}_{2} \mathrm{Cl}_{2}\right.$ /hexanes) providing the pure product as an amorphous white solid ( 0.325 g, 36\% yield). TLC $R_{F}=0.23$ in $50 \% \mathrm{CH}_{2} \mathrm{Cl}_{2} /$ hexanes. ${ }^{1} \mathrm{H}$ NMR $\left(300 \mathrm{MHz}, \mathrm{CDCl}_{3}\right) \delta 1.21-1.33(12 \mathrm{H}, \mathrm{m}), 3.25$ $(1 \mathrm{H}, \mathrm{m}, \mathrm{J}=6.9 \mathrm{~Hz}), 3.38(1 \mathrm{H}, \mathrm{m}, \mathrm{J}=6.9 \mathrm{~Hz}), 6.08(0.4 \mathrm{H}, \mathrm{s}, \mathrm{br}), 6.27(0.5 \mathrm{H}, \mathrm{s}, \mathrm{br}), 7.01(1 \mathrm{H}, \mathrm{d}, \mathrm{J}=7.8 \mathrm{~Hz}), 7.16-$ 7.22 (4H, m), 7.34-7.39 (1H, m). ${ }^{13} \mathrm{C}-\mathrm{NMR}(100 \mathrm{MHz}, \mathrm{CDCl}): \delta 23.5\left(\mathrm{CH}_{3}\right), 23.7\left(\mathrm{CH}_{3}\right), 24.0\left(\mathrm{CH}_{3}\right), 28.7$ (CH), $28.8(\mathrm{CH}), 121.4(\mathrm{CH}), 121.6(\mathrm{CH}), 123.7(\mathrm{CH}), 125.3(\mathrm{CH}), 125.6(\mathrm{CH}), 128.6(\mathrm{CH}), 128.8(\mathrm{CH}), 129.3$ 
(CH), 129.4 (CH),130.3 (C), 130.8 (C), 146.8 (CH), 151.0 (C), 151.2(C), 153.4 (C), 154.8 (C). IR (film): 1801, $1173 \mathrm{~cm}^{-1}$. HRMS (EI): Exact mass calcd for $\mathrm{C}_{19} \mathrm{H}_{23} \mathrm{NO}_{2}[\mathrm{M}]^{+}:$297.1729. Found: 297.1754.<smiles>CC1(C)OB(c2cccc(NC(=O)Oc3ccccc3)c2)OC1(C)C</smiles>

phenyl (3-(4,4,5,5-tetramethyl-1,3,2-dioxaborolan-2-yl)phenyl)carbamate (1i): Synthesized according to general procedure $\mathbf{A}$ using 3-aminophenylboronic acid pinacolate $(0.657 \mathrm{~g}, 3.00 \mathrm{mmol})$, phenyl chloroformate $(0.395 \mathrm{~mL}, 3.15 \mathrm{mmol})$, and $\mathrm{NaHCO}_{3}(0.277 \mathrm{~g}, 0.330 \mathrm{mmol})$. The reaction reached completion within 1 hour. The desired product was dry loaded on to silica gel ( $\mathrm{MeOH}: \mathrm{CH}_{2} \mathrm{Cl}_{2}$ ) and purified by column chromatography $\left(20 \% \mathrm{EtOAc} / \mathrm{CH}_{2} \mathrm{Cl}_{2}\right)$ yielding an amorphous white solid $(0.628 \mathrm{~g}$, $62 \%$ yield). TLC RF $=0.53$ in $20 \% \mathrm{EtOAC} / \mathrm{CH}_{2} \mathrm{Cl}_{2} .{ }^{1} \mathrm{H}-\mathrm{NMR}\left(400 \mathrm{MHz}, \mathrm{CDCl}_{3}\right): \delta 1.32(12 \mathrm{H}, \mathrm{s}), 6.91(1 \mathrm{H}, \mathrm{s}, \mathrm{br})$, 7.15-7.24 (3H, m), 7.32-7.40 (3H, m), 7.53 (1H, d, J=7.3 Hz), 7.69-7.71 (2H, m, br). ${ }^{13} \mathrm{C}-\mathrm{NMR}(75 \mathrm{MHz}$, DMSO- $\left.d_{6}\right): \delta 25.1\left(\mathrm{CH}_{3}\right), 84.1(\mathrm{C}), 122.0(\mathrm{CH}), 122.4(\mathrm{CH}), 124.8(\mathrm{CH}), 125.9(\mathrm{CH}), 128.9(\mathrm{CH}), 129.5(\mathrm{C})$, 129.8 (CH), 138.6 (C), 150.9 (C), 152.2 (C). IR (film): 3276, 1715, $1195 \mathrm{~cm}^{-1}$. HRMS (EI): Exact mass calcd for $\mathrm{C}_{19} \mathrm{H}_{22} \mathrm{BNO}_{4}[\mathrm{M}]^{+}:$339.1642. Found: 339.1658 .<smiles>O=C(NCc1ccccc1)Oc1ccccc1</smiles>

phenyl benzylcarbamate (1j): Synthesized according to general procedure A benzylamine $(0.328 \mathrm{~mL}$, $3.00 \mathrm{mmol})$, phenyl chloroformate $(0.395 \mathrm{~mL}, 3.15 \mathrm{mmol})$, and $\mathrm{NaHCO}_{3}(0.277 \mathrm{~g}, 0.330 \mathrm{mmol})$. The reaction reached completion within 0.3 hours. The desired product was dry loaded onto silica gel $(\mathrm{MeOH})$ and purified by column chromatography $\left(60 \% \mathrm{CH}_{2} \mathrm{Cl}_{2}\right.$ /hexanes) yielding an amorphous white solid $\left(0.565 \mathrm{~g}\right.$, 83\% yield). TLC $\mathrm{R}_{\mathrm{F}}=0.21$ in $60 \% \mathrm{CH}_{2} \mathrm{Cl}_{2} /$ hexanes. ${ }^{1} \mathrm{H}-\mathrm{NMR}\left(400 \mathrm{MHz}, \mathrm{CDCl}_{3}\right): \delta 4.42(1.8 \mathrm{H}$, $\mathrm{d}, \mathrm{J}=6.0 \mathrm{~Hz}), 4.47(0.20 \mathrm{H}, \mathrm{s}, \mathrm{br}), 5.26(0.11 \mathrm{H}, \mathrm{s}, \mathrm{br}), 5.53(0.85 \mathrm{H}, \mathrm{s}, \mathrm{br}), 7.08-7.24(3 \mathrm{H}, \mathrm{m}), 7.28-7.40(7 \mathrm{H}$, m). ${ }^{13} \mathrm{C}-\mathrm{NMR}\left(100 \mathrm{MHz}, \mathrm{CDCl}_{3}\right): \delta 45.3\left(\mathrm{CH}_{2}\right), 121.7(\mathrm{CH}), 125.4(\mathrm{CH}), 127.7(\mathrm{CH}), 127.8(\mathrm{CH}), 128.8(\mathrm{CH})$, $129.4(\mathrm{CH}), 138.2(\mathrm{C}), 151.1(\mathrm{C}), 154.8(\mathrm{C})$. Data is consistent with literature. ${ }^{3}$ 
<smiles>CNC(=O)Oc1ccccc1</smiles>

phenyl methylcarbamate (1k): Synthesized following a previously reported literature procedure. Data is consistent with literature. ${ }^{4}$<smiles>COC(=O)[C@H](NC(=O)Oc1ccccc1)c1ccccc1</smiles>

(S)-methyl 2-((phenoxycarbonyl)amino)-2-phenylacetate (11): Synthesized according to general procedure A (S)-2-phenylglycine methyl ester hydrochloride $(0.605 \mathrm{~g}, 3.00 \mathrm{mmol})$, phenyl chloroformate $(0.395 \mathrm{~mL}, 3.15 \mathrm{mmol})$, and $\mathrm{NaHCO}_{3}(0.554 \mathrm{~g}, 0.660 \mathrm{mmol})$. The reaction reached completion within 0.3 hours. The desired product was dry loaded onto silica gel (MeOH) and purified by column chromatography ( $60 \% \mathrm{CH}_{2} \mathrm{Cl}_{2}$ /hexanes) yielding an amorphous white solid $(0.659 \mathrm{~g}, 77 \%$ yield $) . \mathrm{TLC} \mathrm{R}_{\mathrm{F}}=$ 0.33 in $60 \% \mathrm{CH}_{2} \mathrm{Cl}_{2} /$ hexanes. ${ }^{1} \mathrm{H}-\mathrm{NMR}\left(300 \mathrm{MHz}, \mathrm{CDCl}_{3}\right): \delta 3.72(3 \mathrm{H}, \mathrm{s}), 5.48(1 \mathrm{H}, \mathrm{d}, J=7.5 \mathrm{~Hz}), 6.37(1 \mathrm{H}, \mathrm{d}$, $J=7.4 \mathrm{~Hz}), 7.11-7.20(3 \mathrm{H}, \mathrm{m}), 7.29-7.46(7 \mathrm{H}, \mathrm{m}) .{ }^{13} \mathrm{C}-\mathrm{NMR}\left(75 \mathrm{MHz}, \mathrm{CDCl}_{3}\right): \delta 52.9\left(\mathrm{CH}_{3}\right), 58.1(\mathrm{CH}), 121.6$ (CH), $125.5(\mathrm{CH}), 127.3(\mathrm{CH}), 128.7(\mathrm{CH}), 129.1(\mathrm{CH}), 129.3(\mathrm{CH}), 136.4(\mathrm{C}), 150.9(\mathrm{C}), 153.8(\mathrm{C}), 171.2(\mathrm{C})$. IR (film): 3330, 1737, 1705, $1211 \mathrm{~cm}^{-1}$. HRMS (EI): Exact mass calcd for $\mathrm{C}_{16} \mathrm{H}_{15} \mathrm{NO}_{4}[\mathrm{M}]^{+}: 285.1001$. Found: 285.1066. $[\alpha]^{20}-104^{\circ} \quad(c 0.87, \mathrm{MeCN})$.<smiles>COC(CNC(=O)Oc1ccccc1)OC</smiles>

phenyl (2,2-dimethoxyethyl)carbamate (1m): Synthesized according to general procedure $\mathbf{A}$ using aminoacetaldehyde dimethyl acetal $(0.327 \mathrm{~mL}, 3.00 \mathrm{mmol})$, phenyl chloroformate $(0.395 \mathrm{~mL}, 3.15$ $\mathrm{mmol})$, and $\mathrm{NaHCO}_{3}(0.277 \mathrm{~g}, 3.30 \mathrm{mmol})$. The reaction reached completion within 0.3 hours. The desired product was purified by column chromatography yielding a colorless oil $(0.503 \mathrm{~g}$, $74 \%$ yield). TLC $\mathrm{R}_{\mathrm{F}}=0.36$ in $10 \% \mathrm{EtOAc} / \mathrm{CH}_{2} \mathrm{Cl}_{2} .{ }^{1} \mathrm{H}-\mathrm{NMR}\left(400 \mathrm{MHz}, \mathrm{CDCl}_{3}\right): \delta 3.33-3.41(8 \mathrm{H}, \mathrm{m}), 4.42(1 \mathrm{H}, \mathrm{t}, \mathrm{J}=5.3 \mathrm{~Hz}), 5.56$ 
$(1 \mathrm{H}, \mathrm{s}, \mathrm{br}), 7.07-7.19(3 \mathrm{H}, \mathrm{m}), 7.31(2 \mathrm{H}, \mathrm{t}, \mathrm{J}=7.8 \mathrm{~Hz}) .{ }^{13} \mathrm{C}-\mathrm{NMR}\left(100 \mathrm{MHz}, \mathrm{CDCl}_{3}\right): \delta 42.6(1 \mathrm{C}, \mathrm{s}), 54.3(1 \mathrm{C}, \mathrm{s})$, 102.6 (1C, s), 121.6 (1C, s), 125.3 (1C, s), 129.2 (1C, s), 151.0 (1C, s), 154.8 (1C, s). IR (film): 3318, 1719, $1198 \mathrm{~cm}^{-1}$. HRMS (ESI): Exact mass calcd for $\mathrm{C}_{11} \mathrm{H}_{15} \mathrm{NO}_{4} \mathrm{Na}[\mathrm{M}+\mathrm{Na}]^{+}: 248.0899$. Found: 248.0907 .<smiles>O=C(Nc1ccncc1)Oc1ccccc1</smiles>

phenyl pyridin-4-ylcarbamate (1n): Synthesized according to general procedure B using 4-

aminopyridine $(0.188 \mathrm{~g}, 2.00 \mathrm{mmol})$, phenyl chloroformate $(0.276 \mathrm{~mL}, 2.20 \mathrm{mmol})$, and $\mathrm{Et}_{3} \mathrm{~N}(0.334 \mathrm{~mL}$, $2.40 \mathrm{mmol})$. The reaction was stirred for 16 hours. The crude extract was dry loaded onto silica gel using $\mathrm{MeOH}$ and purified by column chromatography (EtOAc) yielding an amorphous white solid $(0.300 \mathrm{~g}, 70$ $\%$ yield). TLC $R_{F}=0.29$ in EtOAc. ${ }^{1} \mathrm{H}-N M R\left(300 \mathrm{MHz}\right.$, methanol- $\left.d_{4}\right): \delta 8.32(2 \mathrm{H}, \mathrm{d}, J=6.5 \mathrm{~Hz}), 7.52(2 \mathrm{H}, \mathrm{d}$, $J=6.5 \mathrm{~Hz}), 7.36(2 \mathrm{H}, \mathrm{m}), 7.19(3 \mathrm{H}, \mathrm{m}) .{ }^{13} \mathrm{C}-\mathrm{NMR}\left(400 \mathrm{MHz}\right.$, Acetone- $\left.d_{6}\right): \delta 113.7(\mathrm{CH}), 122.8(\mathrm{CH}), 126.7$ (CH), $130.4(\mathrm{CH}), 147.0$ (C), $151.5(\mathrm{CH}), 151.8$ (C), 152.5 (C). IR (film): 1751, $1188 \mathrm{~cm}^{-1}$. HRMS (EI): Exact mass calcd for $\mathrm{C}_{12} \mathrm{H}_{11} \mathrm{~N}_{2} \mathrm{O}_{2}[\mathrm{M}+\mathrm{H}]^{+}:$215.0821. Found: 215.0826 .<smiles>O=C(Nc1ccc(Cl)nc1)Oc1ccccc1</smiles>

phenyl (6-chloropyridin-3-yl)carbamate (10): Synthesized according to general procedure B using 6chloro-pyridin-3-ylamine $(0.385 \mathrm{~g}, 3.00 \mathrm{mmol})$, phenyl chloroformate $(0.395 \mathrm{~mL}, 3.15 \mathrm{mmol})$, and $\mathrm{Et}_{3} \mathrm{~N}$ $(0.460 \mathrm{~mL}, 3.30 \mathrm{mmol})$. The reaction reached completion within 2 hours. The crude mixture was dry loaded onto silica gel (acetone) and purified using column chromatography ( $5 \% \mathrm{EtOAc} / \mathrm{CH}_{2} \mathrm{Cl}_{2}$ ) yielding an amorphous white solid ( $0.297 \mathrm{~g}, 40 \%$ yield). TLC $\mathrm{R}_{\mathrm{F}}=0.33$ in $5 \% \mathrm{EtOAC} / \mathrm{CH}_{2} \mathrm{Cl}_{2} .{ }^{1} \mathrm{H}-\mathrm{NMR}(400 \mathrm{MHz}$, Acetone- $\left.d_{6}\right)$ : $\delta 7.21-7.28(3 \mathrm{H}, \mathrm{m}), 7.39-7.44(3 \mathrm{H}, \mathrm{m}), 8.10(1 \mathrm{H}, \mathrm{dd}, J=2.8 \mathrm{~Hz}, 8.7 \mathrm{~Hz}), 8.59(1 \mathrm{H}, \mathrm{d}, J=2.7 \mathrm{~Hz})$, $9.52(1 \mathrm{H}, \mathrm{s}) .{ }^{13} \mathrm{C}-\mathrm{NMR}\left(100 \mathrm{MHz}\right.$, Acetone- $\left.d_{6}\right): \delta 121.7(\mathrm{CH}), 124.1(\mathrm{CH}), 125.6(\mathrm{CH}), 128.9(\mathrm{CH}), 129.3$ (CH), 135.2 (C), 140.0 (CH), 144.5 (C), 150.9 (C), 151.9 (C). IR (film): 1741, $1193 \mathrm{~cm}^{-1}$. HRMS (EI): Exact mass calcd for $\mathrm{C}_{12} \mathrm{H}_{9} \mathrm{~N}_{2} \mathrm{O}_{2} \mathrm{Cl}[\mathrm{M}]^{+}:$248.0353. Found: 248.0334 . 


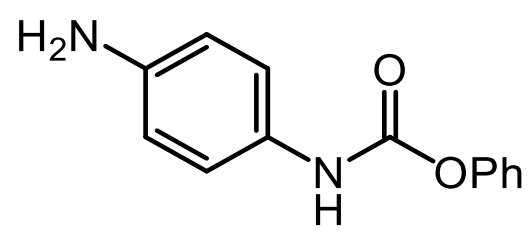

phenyl (4-aminophenyl)carbamate (1p): Synthesized according to general procedure B using with pphenylenediamine $(1.85 \mathrm{~g}, 11.0 \mathrm{mmol})$, phenyl chloroformate $(1.25 \mathrm{~mL}, 10.0 \mathrm{mmol})$, and $\mathrm{Et}_{3} \mathrm{~N}(1.53 \mathrm{~mL}$, $11.0 \mathrm{mmol})$ and $\mathrm{CH}_{2} \mathrm{Cl}_{2}(100 \mathrm{~mL})$. The reaction time was for 16 hours. The crude reaction mixture was dry loaded onto silica ( $\mathrm{MeOH}$ ) and purified by column chromatography $\left(80 \% \mathrm{CH}_{2} \mathrm{Cl}_{2} / \mathrm{EtOAc}\right)$ yielding a pure white/yellow amorphous solid (1.27 g, $56 \%$ yield). TLC $\mathrm{R}_{\mathrm{F}}=0.53$ in $80 \% \mathrm{CH}_{2} \mathrm{Cl}_{2} / \mathrm{EtOAc}$. $1 \mathrm{H}-\mathrm{NMR}$ (400 MHz, Acetone- $\left.d_{6}\right): \delta 3.58(1 \mathrm{H}, \mathrm{s}), 6.62(1 \mathrm{H}, \mathrm{q}, J=2.9 \mathrm{~Hz}), 6.86(1 \mathrm{H}, \mathrm{s}), 7.19(1 \mathrm{H}, \mathrm{m}, J=5.4 \mathrm{~Hz}), 7.36$ $(1 \mathrm{H}, \mathrm{t}, J=7.9 \mathrm{~Hz}) \cdot{ }^{13} \mathrm{C}-\mathrm{NMR}\left(100 \mathrm{MHz}\right.$, Acetone- $\left.d_{6}\right): \delta 114.5(\mathrm{CH}), 120.5(\mathrm{CH}), 121.8(\mathrm{CH}), 124.9(\mathrm{CH}), 128.4$ (C) 129.1 (CH), 144.6 (C), 151.4 (C), 151.9 (C). IR (film): 3450, 3367, 3308, 1706, $1196 \mathrm{~cm}^{-1}$. HRMS (EI): Exact mass calcd for $\mathrm{C}_{13} \mathrm{H}_{12} \mathrm{~N}_{2} \mathrm{O}_{2}[\mathrm{M}]^{+}: 228.0898$. Found: 228.0901 .<smiles>Nc1cccc(NC(=O)Oc2ccccc2)c1</smiles>

phenyl (3-aminophenyl)carbamate (1q): Synthesized according to general procedure B using with mphenylenediamine $(0.324 \mathrm{~g}, 3.00 \mathrm{mmol})$, phenyl chloroformate $(0.376 \mathrm{~g}, 3.00 \mathrm{mmol})$, and $\mathrm{Et}_{3} \mathrm{~N}(0.460$ $\mathrm{mL}, 3.30 \mathrm{mmol})$. The reaction was complete within 16 hours. The crude mixture was dry loaded onto silica gel $(\mathrm{MeOH})$ and purified by column chromatography $\left(10 \% \mathrm{EtOAc} / \mathrm{CH}_{2} \mathrm{Cl}_{2}\right)$ yielding an amorphous yellow/white solid (0.393 g, 57\% yield). TLC RF $=0.25$ in $10 \% \mathrm{EtOAc} / \mathrm{CH}_{2} \mathrm{Cl}_{2} .{ }^{1} \mathrm{H}-\mathrm{NMR}$ (400 MHz, Acetone$\left.d_{6}\right): \delta 4.66(1 \mathrm{H}, \mathrm{s}, \mathrm{br}), 6.38(1 \mathrm{H}, \mathrm{d}, J=7.1 \mathrm{~Hz}), 6.79(1 \mathrm{H}, \mathrm{d}, J=8.0 \mathrm{~Hz}), 6.96-7.01(2 \mathrm{H}, \mathrm{m}), 7.16-7.23(3 \mathrm{H}, \mathrm{m})$, $7.39(2, \mathrm{t}, J=2.6 \mathrm{~Hz}), 8.95(1 \mathrm{H}, \mathrm{s}, \mathrm{br}) .{ }^{13} \mathrm{C}-\mathrm{NMR}\left(100 \mathrm{MHz}\right.$, Acetone- $\left.d_{6}\right): \delta 104.5(\mathrm{CH}), 107.2(\mathrm{CH}), 109.5$ (CH), $121.8(\mathrm{CH}), 125.1(\mathrm{CH}), 129.1(\mathrm{CH}), 129.2(\mathrm{CH}), 139.5(\mathrm{C}), 149.1(\mathrm{C}), 151.2(\mathrm{C}), 151.5(\mathrm{C}) . \mathrm{IR}$ (film): $3403,3314,1724,1196 \mathrm{~cm}^{-1}$. HRMS (El): Exact mass calcd for $\mathrm{C}_{13} \mathrm{H}_{12} \mathrm{~N}_{2} \mathrm{O}_{2}$ [M] ${ }^{+}: 228.0899$. Found: 228.0898 .<smiles>O=C(Nc1ccc(Nc2ccccc2)cc1)Oc1ccccc1</smiles> 
phenyl (4-(phenylamino)phenyl)carbamate (1r): Synthesized according to general procedure B using with $N$-phenyl-p-phenylenediamine $(0.368 \mathrm{~g}, 2.00 \mathrm{mmol})$, phenyl chloroformate $(0.260 \mathrm{~mL}, 2.10 \mathrm{mmol})$, and $\mathrm{Et}_{3} \mathrm{~N}(0.306 \mathrm{~mL}, 2.20 \mathrm{mmol})$. The reaction was stirred for 16 hours. The crude mixture was dried loaded onto silica $\left(\mathrm{CH}_{2} \mathrm{Cl}_{2} / \mathrm{MeOH}\right)$ and purified by column chromatography $\left(100 \% \mathrm{CH}_{2} \mathrm{Cl}_{2}\right)$ yielding an amorphous yellow solid ( $0.447 \mathrm{~g}, 73 \%$ yield). $\mathrm{TLC} \mathrm{R}_{\mathrm{F}}=0.39$ in $\mathrm{CH}_{2} \mathrm{Cl}_{2} \cdot{ }^{1} \mathrm{H}-\mathrm{NMR}\left(400 \mathrm{MHz}, \mathrm{CDCl}_{3}\right): \delta 5.64$ $(1 \mathrm{H}, \mathrm{s}, \mathrm{br}), 6.87-6.91(2 \mathrm{H}, \mathrm{m}), 6.98-7.04(4 \mathrm{H}, \mathrm{m}), 7.17-7.26(6 \mathrm{H}, \mathrm{m}), 7.29-7.34(2 \mathrm{H}, \mathrm{m}), 7.36-7.41(2 \mathrm{H}, \mathrm{t}$, J=7.4 Hz). ${ }^{13} \mathrm{C}-\mathrm{NMR}\left(400 \mathrm{MHz}, \mathrm{CDCl}_{3}\right): \delta 117.2(\mathrm{CH}), 119.4(\mathrm{CH}), 120.6(\mathrm{CH}), 120.7(\mathrm{CH}), 121.7(\mathrm{CH}), 125.7$ (CH), $129.4(\mathrm{CH}), 129.4(\mathrm{CH}), 131.1$ (C), 139.4 (C), 143.7 (C), 150.7 (C), 152.0 (C). IR (film): 3410, 3315, 1726, $1193 \mathrm{~cm}^{-1}$. HRMS (EI): Exact mass calcd for $\mathrm{C}_{19} \mathrm{H}_{16} \mathrm{~N}_{2} \mathrm{O}_{2}[\mathrm{M}]^{+}: 304.1212$. Found: 304.1188.

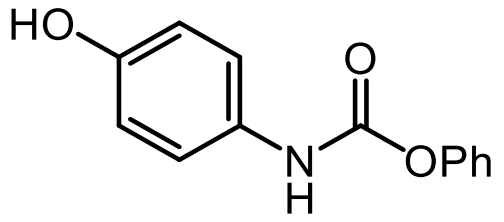

phenyl (4-hydroxyphenyl)carbamate (1s): Synthesized according to general procedure A using 4-aminophenol (0.327 g, $3.00 \mathrm{mmol})$, phenyl chloroformate $(0.395 \mathrm{~mL}, 3.15 \mathrm{mmol})$, and $\mathrm{NaHCO}_{3}(0.277 \mathrm{~mL}, 3.30$ $\mathrm{mmol})$. The reaction reached completion within 0.5 hours. The crude mixture was purified using column chromatography ( $10 \% \mathrm{EtOAC} / \mathrm{CH}_{2} \mathrm{Cl}_{2}$ ) yielding an amorphous white solid $\left(0.579 \mathrm{~g}, 84 \%\right.$ yield). $\mathrm{TLC} \mathrm{R}_{\mathrm{F}}=$ 0.34 in $10 \%$ EtOAc/ $\mathrm{CH}_{2} \mathrm{Cl}_{2} .{ }^{1} \mathrm{H}-\mathrm{NMR}\left(300 \mathrm{MHz}\right.$, Acetone- $\left.d_{6}\right): \delta 6.82(2 \mathrm{H}, \mathrm{d}, J=8.9 \mathrm{~Hz}), 7.16-7.21(3 \mathrm{H}, \mathrm{m})$, 7.33-7.43 $(4 \mathrm{H}, \mathrm{m}), 8.19(1 \mathrm{H}, \mathrm{s}), 8.87(1 \mathrm{H}, \mathrm{s}) .{ }^{13} \mathrm{C}-\mathrm{NMR}\left(100 \mathrm{MHz}\right.$, Acetone- $\left.d_{6}\right): \delta 115.7(\mathrm{CH}), 120.9(\mathrm{CH})$, 122.1 (CH), 125.4 (CH), 129.5 (CH), 131.0 (C), 151.5 (C), 152.3 (C), 153.8 (C). IR (film): 3350, 1719, 1196 $\mathrm{cm}^{-1}$. HRMS (EI): Exact mass calcd for $\mathrm{C}_{13} \mathrm{H}_{11} \mathrm{NO}_{3}[\mathrm{M}]^{+}: 229.0739$. Found: 229.0743.

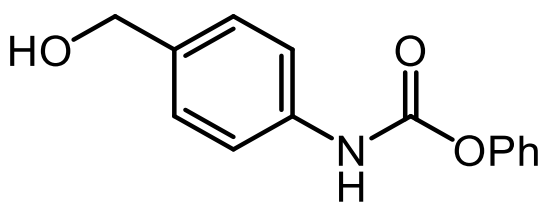

phenyl (4-(hydroxymethyl)phenyl)carbamate (1t): Synthesized according to general procedure A using 4-aminobenzenemethanol $(0.369 \mathrm{~g}, 3.00 \mathrm{mmol})$, phenyl chloroformate $(0.395 \mathrm{~mL}, 3.15 \mathrm{mmol})$, and $\mathrm{NaHCO}_{3}(0.277 \mathrm{~g}, 3.30 \mathrm{mmol})$. The reaction reached completion within 0.3 hours. The product was recrystallized twice (50\% EtOAc/ hexanes) yielding a white solid $\left(0.547 \mathrm{~g}, 75 \%\right.$ yield). TLC $R_{F}=0.63$ in $50 \% \mathrm{CH}_{2} \mathrm{Cl}_{2}$ /EtOAc. ${ }^{1} \mathrm{H}-\mathrm{NMR}(400 \mathrm{MHz}, \mathrm{CDCl}): \delta 1.79(1 \mathrm{H}, \mathrm{s}, \mathrm{br}), 4.62(2 \mathrm{H}, \mathrm{d}, \mathrm{J}=4.4 \mathrm{~Hz}), 7.06(1 \mathrm{H}, \mathrm{s}, \mathrm{br})$, $7.17(2 \mathrm{H}, \mathrm{d}, J=7.6 \mathrm{~Hz}), 7.22(1 \mathrm{H}, \mathrm{t}, J=7.3 \mathrm{~Hz}), 7.30(2 \mathrm{H}, \mathrm{d}, J=8.5 \mathrm{~Hz}), 7.35-7.42(4 \mathrm{H}, \mathrm{m}) .{ }^{13} \mathrm{C}-\mathrm{NMR}(400 \mathrm{MHz}$, $\left.\mathrm{CDCl}_{3}\right): \delta 64.8\left(\mathrm{CH}_{2}\right), 118.9(\mathrm{CH}), 121.7(\mathrm{CH}), 125.8(\mathrm{CH}), 128.0(\mathrm{CH}), 129.4(\mathrm{CH}), 136.5(\mathrm{C}), 136.9(\mathrm{C})$, 150.6 (C), 151.7 (C). IR (film): 3364, 1725, $1195 \mathrm{~cm}^{-1}$. HRMS (EI): Exact mass calcd for $\mathrm{C}_{14} \mathrm{H}_{13} \mathrm{NO}_{3}[\mathrm{M}]^{+}$: 243.0895. Found: 243.0912. 


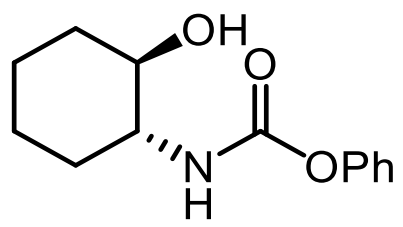

phenyl ((1R,2R)-2-hydroxycyclohexyl)carbamate (1u): Synthesized according to general procedure A using (1S,2S)-trans-2-aminocyclohexanol $(0.346 \mathrm{~g}, 3.00 \mathrm{mmol})$, phenyl chloroformate $(0.395 \mathrm{~mL}, 3.15$ $\mathrm{mmol})$, and $\mathrm{NaHCO}_{3}(0.277 \mathrm{~g}, 3.3 \mathrm{mmol})$. The reaction reached completion within 0.3 hours. The reaction was diluted with EtOAc $(50 \mathrm{~mL})$ and extracted with water $(1 \times 30 \mathrm{~mL})$ and brine $(1 \times 30 \mathrm{~mL})$. The organic layer was dried over sodium sulfate, concentrated under reduced pressure. The crude mixture was purified by column chromatography $\left(25 \% \mathrm{EtOAc} / \mathrm{CH}_{2} \mathrm{Cl}_{2}\right)$ yielding an amorphous white solid ( 0.523 g, 74\% yield). TLC RF $=0.31$ in $25 \%$ EtOAc $/ \mathrm{CH}_{2} \mathrm{Cl}_{2} .{ }^{1} \mathrm{H}-\mathrm{NMR}\left(400 \mathrm{MHz}, \mathrm{CDCl}_{3}\right): \delta 1.13-1.36(4 \mathrm{H}, \mathrm{m}), 1.66-$ $1.73(1 \mathrm{H}, \mathrm{m}), 2.01-2.08(1 \mathrm{H}, \mathrm{m}), 2.80(1 \mathrm{H}, \mathrm{s}), 3.30-3.45(2 \mathrm{H}, \mathrm{m}), 5.18(1 \mathrm{H}, \mathrm{d}, J=4.6 \mathrm{~Hz}), 7.11(2 \mathrm{H}, \mathrm{d}, J=7.5$ $\mathrm{Hz}), 7.17(1 \mathrm{H}, \mathrm{t}, J=7.4 \mathrm{~Hz}), 7.33(1 \mathrm{H}, \mathrm{t}, \mathrm{J}=8.3 \mathrm{~Hz}) .{ }^{13} \mathrm{C}-\mathrm{NMR}\left(400 \mathrm{MHz}, \mathrm{CDCl}_{3}\right): \delta 24.1\left(\mathrm{CH}_{2}\right), 24.6\left(\mathrm{CH}_{2}\right), 31.6$ $\left(\mathrm{CH}_{2}\right), 34.3\left(\mathrm{CH}_{2}\right), 57.3(\mathrm{CH}), 74.7(\mathrm{CH}), 121.6(\mathrm{CH}), 125.4(\mathrm{CH}), 129.3(\mathrm{CH}), 150.9(\mathrm{C}), 155.6(\mathrm{C})$. IR (film): $3425,3243,1700,1198 \mathrm{~cm}^{-1}$. HRMS (El): Exact mass calcd for $\mathrm{C}_{13} \mathrm{H}_{17} \mathrm{NO}_{3}[\mathrm{M}]^{+}:$235.1208. Found: 235.1193. $[\alpha]^{20} \mathrm{D}+16.2^{\circ}(c 1.51, \mathrm{MeOH})$.

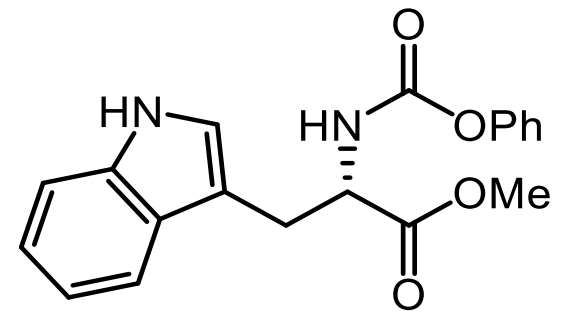

(S)-methyl 3-(1H-indol-3-yl)-2-((phenoxycarbonyl)amino)propanoate (1v): Synthesized according to general procedure $\mathbf{A}$ using (L)-tryptophan methyl ester hydrochloride $(0.764 \mathrm{~mL}, 3.00 \mathrm{mmol})$, phenyl chloroformate $(0.395 \mathrm{~mL}, 3.15 \mathrm{mmol})$, and $\mathrm{NaHCO}_{3}(0.529 \mathrm{~g}, 6.3 \mathrm{mmol})$. The reaction reached completion within 0.5 hours. The desired product was purified by column chromatography (30\% EtOAc) hexanes) yielding an amorphous white solid $(0.783 \mathrm{~g}, 77 \%)$. TLC $\mathrm{R}_{\mathrm{F}}=0.23$ in $30 \% \mathrm{EtOAc} /$ hexanes. ${ }^{1} \mathrm{H}-$ NMR $\left(400 \mathrm{MHz}\right.$, Acetone- $\left.d_{6}\right): \delta$ 3.25-3.45 $(2 \mathrm{H}, \mathrm{m}), 3.70(3 \mathrm{H}, \mathrm{s}), 4.62-4.69(1 \mathrm{H}, \mathrm{m}), 6.99(1 \mathrm{H}, \mathrm{d}, J=8.1 \mathrm{~Hz})$, 7.05-7.19 (5H, m), 7.27-7.35 (3H, m), 7.41 (1H, d, J=8.0 Hz), 7.65 (1H, d, J=7.8 Hz), $10.08(1 \mathrm{H}, \mathrm{s}) .{ }^{13} \mathrm{C}-\mathrm{NMR}$ (100 MHz, Acetone- $\left.d_{6}\right): \delta 27.5\left(\mathrm{CH}_{2}\right), 51.7\left(\mathrm{CH}_{3}\right), 55.3(\mathrm{CH}), 109.9(\mathrm{C}), 111.5(\mathrm{CH}), 118.3(\mathrm{CH}), 119.0(\mathrm{CH})$, 121.5 (CH), 121.7 (CH), 123.8 (CH), $125.1(\mathrm{CH}), 127.7(\mathrm{C}), 129.2(\mathrm{CH}), 136.8(\mathrm{C}), 151.5(\mathrm{C}), 154.3(\mathrm{C}), 172.3$ (C). IR (film): 3405, 3328, 1734, 1709, 1214, $1182 \mathrm{~cm}^{-1}$. HRMS (EI): Exact mass calcd for $\mathrm{C}_{19} \mathrm{H}_{18} \mathrm{~N}_{2} \mathrm{O}_{4}[\mathrm{M}]^{+}$: 338.1267. Found: 338.1258 . $[\alpha]^{20}{ }_{D}+18.9^{\circ}$ (c 1.20, MeCN). Chiral HPLC: ChiralPak column AD-H $i-$ $\mathrm{PrOH} / \mathrm{Hexane}=15: 85,1.0 \mathrm{~mL} / \mathrm{min}, 230 \mathrm{~nm}, t=20.2 \mathrm{~min}$. 


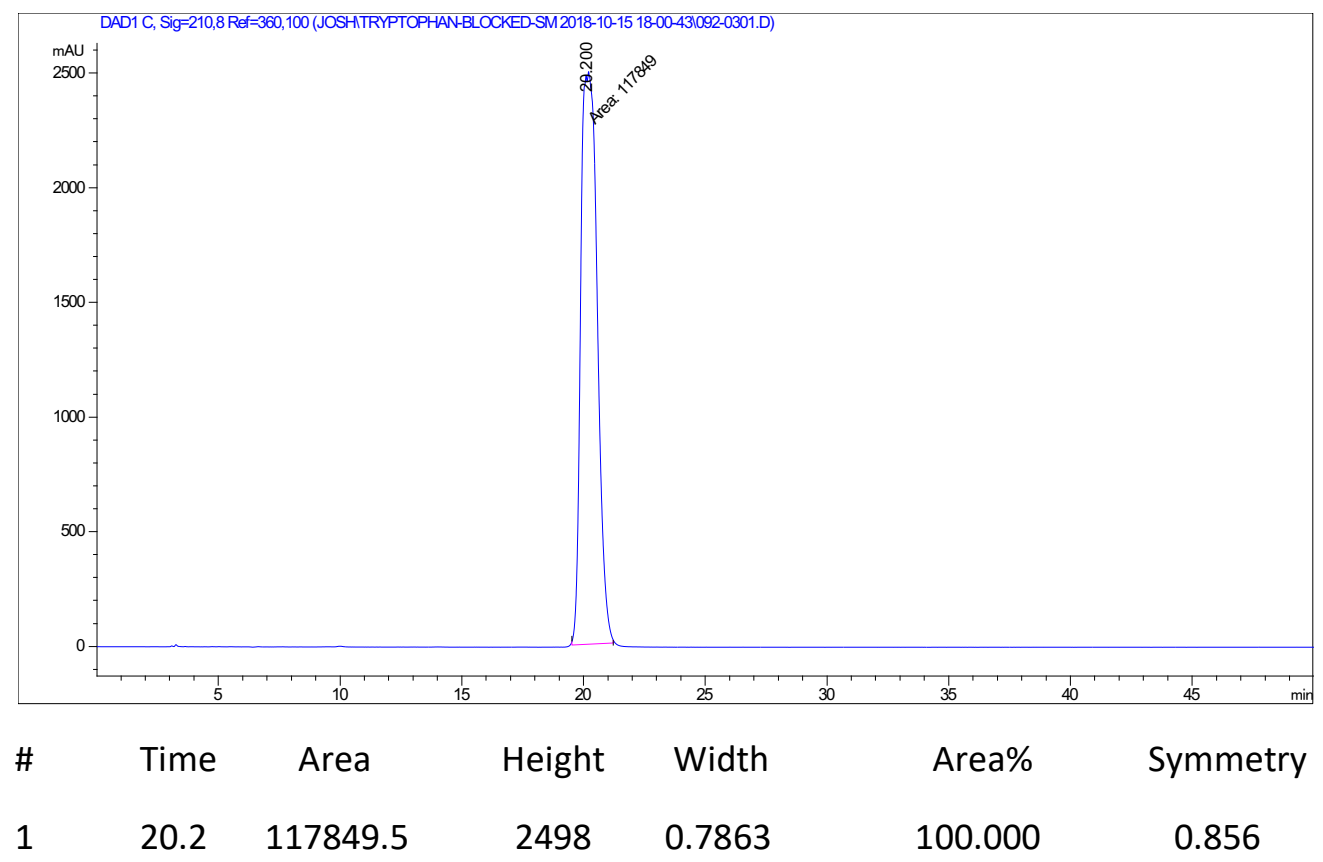

Figure S1. HPLC trace and data of $\mathbf{1 v}$ showing presence of single enantiomer.

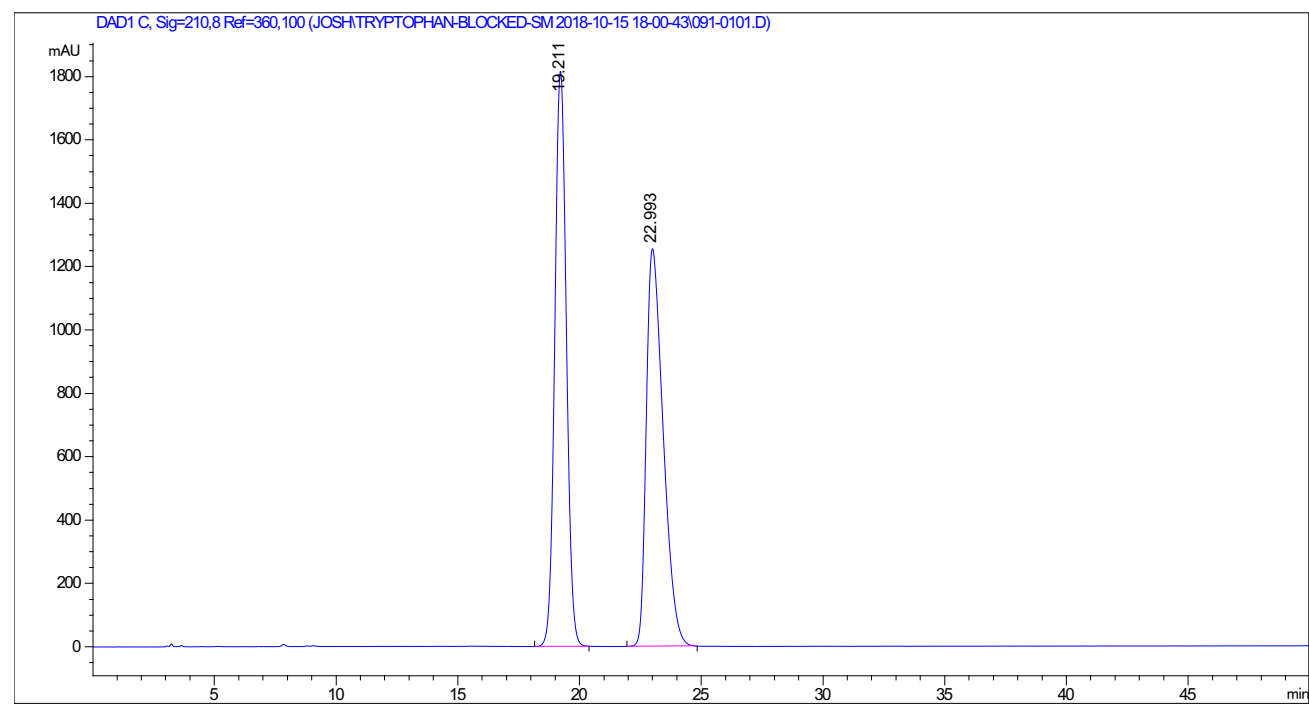

\# $\quad$ Time Area Height Width Area\% Symmetry

$\begin{array}{lllllll}1 & 19.211 & 59954.9 & 1815.3 & 0.5153 & 49.743 & 0.924\end{array}$

$\begin{array}{lllllll}2 & 22.993 & 60575.4 & 1255.1 & 0.7131 & 50.257 & 0.556\end{array}$

Figure S2. HPLC trace and data of racemic $\mathbf{1 v}$. 


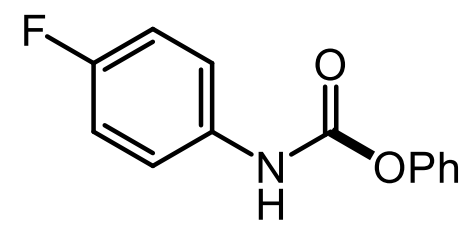

phenyl (4-fluorophenyl)carbamate (1w): Synthesized according to general procedure $\mathbf{A}$ using 4fluoroaniline $(0.947 \mathrm{~mL}, 10.0 \mathrm{mmol})$, phenyl chloroformate $(1.32 \mathrm{~mL}, 10.5 \mathrm{mmol})$, and $\mathrm{NaHCO}_{3}(0.924 \mathrm{~g}$, $10.5 \mathrm{mmol}$ ). The reaction reached completion within 1 hour. The desired product was recrystallized (20\% EtOAC/ hexanes) 3 times yielding a white solid ( $2.02 \mathrm{~g}, 87 \%$ yield). TLC $\mathrm{R}_{\mathrm{F}}=0.73$ in $\mathrm{CH}_{2} \mathrm{Cl}_{2} .{ }^{1} \mathrm{H}-\mathrm{NMR}$ (400 MHz, Acetone- $\left.d_{6}\right): \delta 7.11(2 \mathrm{H}, \mathrm{m}, J=8.8 \mathrm{~Hz}), 7.18-7.25(3 \mathrm{H}, \mathrm{m}), 7.40(2 \mathrm{H}, \mathrm{t}, J=7.5), 7.60-7.64(2 \mathrm{H}, \mathrm{m})$, $9.22(1 \mathrm{H}, \mathrm{s}, \mathrm{br}) .{ }^{13} \mathrm{C}-\mathrm{NMR}\left(100 \mathrm{MHz}\right.$, Acetone- $\left.d_{6}\right): \delta 115.4(\mathrm{CH}, \mathrm{d}, J=22.6 \mathrm{~Hz}), 120.3(\mathrm{CH}), 121.8(\mathrm{CH}), 125.3$ (CH), $129.3(\mathrm{CH}), 135.2(\mathrm{C}), 151.5(\mathrm{C}, \mathrm{d}, \mathrm{J}=80.6 \mathrm{~Hz}), 157.5$ (C), 159.9 (C). Data is consistent with literature. ${ }^{5}$

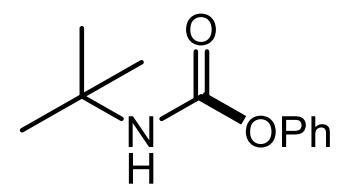

phenyl tert-butylcarbamate (1x): An oven-dried microwave vial equipped with a magnetic stir bar and fitted with a microwave cap was charged with diphenyl carbonate $(3.21 \mathrm{~g}, 15.0 \mathrm{mmol})$ and heated to 70 ${ }^{\circ} \mathrm{C}$ until the diphenyl carbonate had turned into a liquid. Subsequently, the corresponding amine (1.05 $\mathrm{mL}, 10.0 \mathrm{mmol}$ ) was added dropwise to the vial and stirred for an additional 10 minutes at $70{ }^{\circ} \mathrm{C}$. The reaction was run for $16 \mathrm{~h}$ and then cooled to room temperature, diluted with EtOAc $(15 \mathrm{~mL})$ and extracted $1 \mathrm{M} \mathrm{NaOH}(2 \times 20 \mathrm{~mL})$, saturated $\mathrm{NaHCO}_{3}(1 \times 20 \mathrm{~mL})$, and brine $(1 \times 20 \mathrm{~mL})$. The organic layer was collected, dried over $\mathrm{Na}_{2} \mathrm{SO}_{4}$, and concentrated under reduced pressure. The crude mixture was purified by silica gel chromatography (10\% EtOAc/hexanes) yielding the desired product as a crystalline white solid (0.809 g, 42\%). Rf 0.81 (20\% EtOAc/hexanes). $\left.{ }^{1} \mathrm{H} \mathrm{NMR} \mathrm{(500} \mathrm{MHz,} \mathrm{CDCl}_{3}\right) \delta 7.38-7.31(\mathrm{~m}, 2 \mathrm{H})$, $7.21-7.14(\mathrm{~m}, 1 \mathrm{H}), 7.12(\mathrm{dd}, J=8.5,1.2 \mathrm{~Hz}, 2 \mathrm{H}), 5.06-4.91(\mathrm{br} \mathrm{s}, 1 \mathrm{H}), 1.39(\mathrm{~s}, 9 \mathrm{H}) .{ }^{13} \mathrm{C} \mathrm{NMR}(126 \mathrm{MHz}$, $\left.\mathrm{CDCl}_{3}\right) \delta 152.9(\mathrm{C}), 151.1(\mathrm{C}), 129.4(\mathrm{CH}), 125.2(\mathrm{CH}), 121.9(\mathrm{CH}), 51.0(\mathrm{C}), 29.0\left(\mathrm{CH}_{3}\right)$. Data is consistent with literature. ${ }^{6}$<smiles>O=C(Nc1ccccc1)N1CCOCC1</smiles><smiles>O=C(Nc1ccccc1)N(Cc1ccccc1)Cc1ccccc1</smiles><smiles>CC(C)(C)C(=O)NC(=O)Nc1ccccc1</smiles><smiles>O=C(Nc1ccccc1)Nc1ccccc1</smiles><smiles>CCCCC(C)(C)N(C(=O)Nc1ccccc1)c1ccccc1</smiles>

Ureas $5 \mathbf{a a}^{7}, \mathbf{5} \mathbf{a b}^{8}, \mathbf{5} \mathbf{a c}^{9}, \mathbf{5} \mathbf{a d}^{10}$, and $\mathbf{5} \mathbf{a e}^{11}$ were synthesized from literature procedures. 


\section{5-Selected additional optimization data (Tables S1-S12)}

Optimizations were conducted following general procedures $C$ and $D$ (see section below for detailed procedures) on $0.200 \mathrm{mmol}$ scale. Organoboron reagent $2 \mathrm{a}$ was used as received from the commercial supplier as a mixture of boronic acid:boroxine 2.1:1. ${ }^{12}$ Yields were determined by ${ }^{1} \mathrm{H}-\mathrm{NMR}$ of the crude reactions using 1,3,5-trimethoxybenzene as an internal standard.

Table S1. Effect of temperature under base free conditions for the reaction of substrate $\mathbf{1 a}$<smiles>O=C(Nc1ccccc1)Oc1ccccc1</smiles>

1

2

3

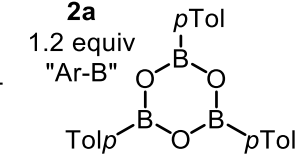

Temperature

$80^{\circ} \mathrm{C}$

$100^{\circ} \mathrm{C}$

$120^{\circ} \mathrm{C}$

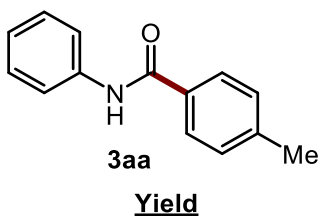

$11 \%$

$24 \%$

$80 \%$

Table S2. Effect of concentration on the reaction of substrate 1a

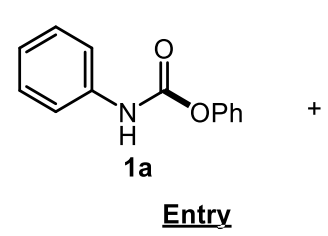

1

2

3

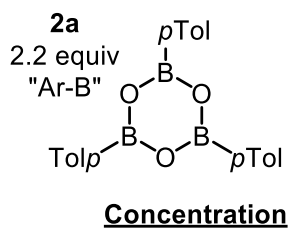

$0.05 \mathrm{M}$

$0.1 \mathrm{M}$

$0.5 \mathrm{M}$
$[\mathrm{Rh}(\mathrm{OH})(\mathrm{cod})]_{2}(2.5 \mathrm{~mol} \%)$

$$
\underset{50^{\circ} \mathrm{C}, \mathrm{THF}}{\stackrel{\mathrm{Et}_{3} \mathrm{~N}(1 \text { equiv.) }}{\longrightarrow}}
$$

$30 \mathrm{~h}$

$20 \mathrm{~h}$

$5 \mathrm{~h}$

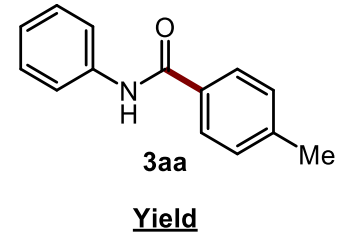

$28 \%^{a}$

$94 \%$

$96 \%$

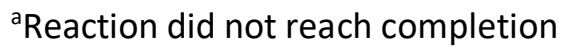


Table S3. Effect of solvent (0.5 M) on the reaction of substrate 1a

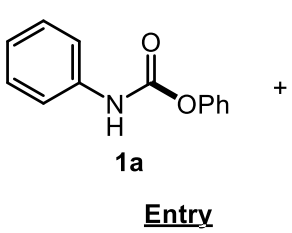

1

2

3

4

5

6

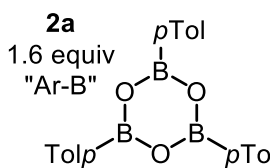

Solvent

$\mathrm{PhCF}_{3}$

$\mathrm{CH}_{2} \mathrm{Cl}_{2}$

DMSO

$\mathrm{MeOH}$

MeCN

THF

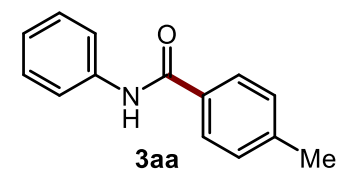

Yield

$16 \%$

$15 \%$

$8 \%$

$10 \%^{a}$

$43 \%$

$89 \%$

${ }^{\mathrm{a}} 90 \%$ of the methanol addition adduct

Table S4. Effect of catalyst loadings on the reaction of substrate $\mathbf{1 a}$

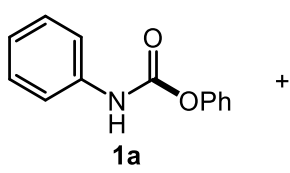

Entry

1

2

3

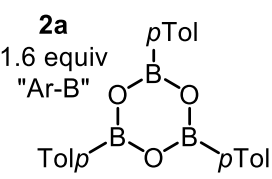

Loading

$2.5 \mathrm{~mol} \%$

$1 \mathrm{~mol} \%$

$0.5 \mathrm{~mol} \%$
$[\mathrm{Rh}(\mathrm{OH})(\mathrm{cod})]_{2}$

$\underset{\text { THF } 0.5 \mathrm{M}, 50^{\circ} \mathrm{C}}{\stackrel{\mathrm{Et}_{3} \mathrm{~N}(1 \text { equiv.) }}{\longrightarrow}}$

Iime

$5 \mathrm{~h}$

$5 \mathrm{~h}$

$24 \mathrm{~h}$

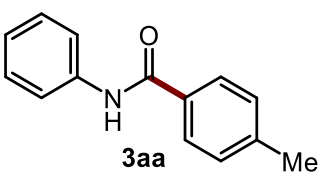

Yield

$95 \%$

$89 \%$

$69 \%^{a}$

${ }^{\text {a }}$ The reaction did not reach completion 
Table S5. Effect of base on substrate for the reaction of substrate 1a

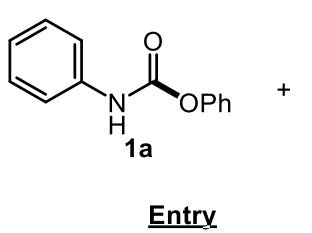

1

2

3

4

5

6

7

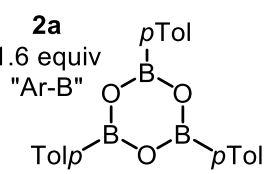

Base

DBU

$\mathrm{Cs}_{2} \mathrm{CO}_{3}$

$\mathrm{N}$-methyl morpholine

DIPEA

$\mathrm{K}_{2} \mathrm{CO}_{3}$

$\mathrm{Et}_{3} \mathrm{~N}$ (0.2 equiv.)

$\mathrm{Et}_{3} \mathrm{~N}$

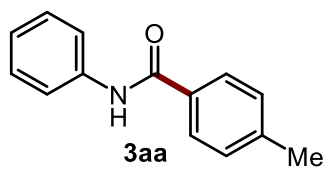

Yield

$0 \%$

$24 \%$

$0 \%$

$26 \%$

$0 \%$

$<5 \%$

$89 \%$

Table S6. Ligand screen for the reaction of substrate $\mathbf{1 n}$

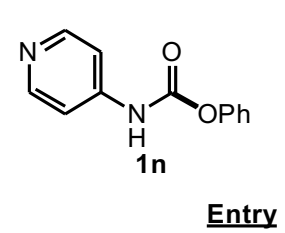

1

2

3

4

5

6

7

8

9

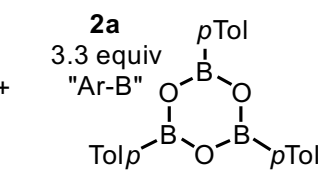

$\underline{\text { Ligand }}^{\mathrm{a}}$

None

Binap

DPPE

$\mathrm{P}(\mathrm{OEt})_{3}$

$\mathrm{PPh}_{3}$

DPPF

Binap ${ }^{b}$

Binapc

None ${ }^{c}$

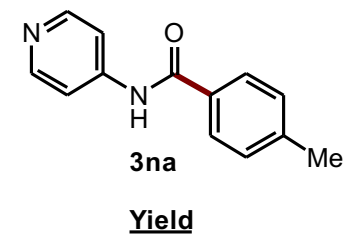

$3 \%$

$8 \%$

$4 \%$

$0 \%$

$0 \%$

$0 \%$

$4 \%$

$15 \%$

$51 \%$

${ }^{\mathrm{a}} 2: 1$ ligand:Rh ratio for monodentate ligand. 1.1:1 ligand:Rh ratio for bidentate ratio. ${ }^{\mathrm{b}} 5: 1$ Ligand:Rh. ${ }^{\mathrm{c}}$ No base, $120^{\circ} \mathrm{C}, 3 \mathrm{~h}$. 
Table S7. Effect of temperature on the reaction of substrate 1p

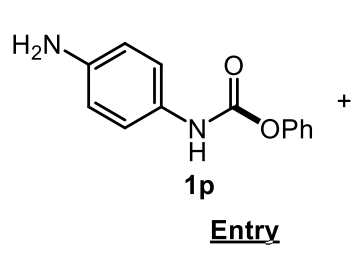

1

2

3

4

5

6

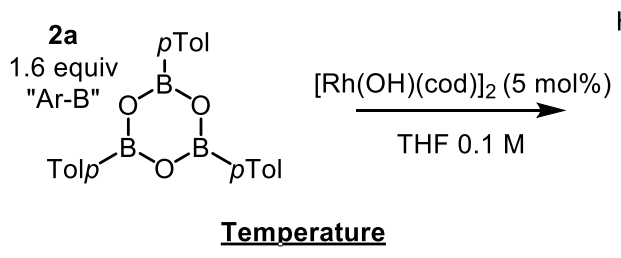

$80^{\circ} \mathrm{C}$

$100^{\circ} \mathrm{C}$

$120^{\circ} \mathrm{C}$

$100^{\circ} \mathrm{C}$

$50^{\circ} \mathrm{C}$

$50^{\circ} \mathrm{C}$

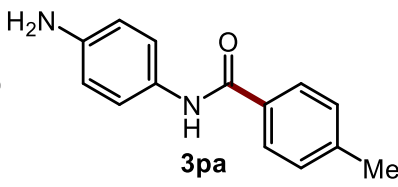

Yield

$34 \%$

$75 \%$

$25 \%$

$41 \%{ }^{a}$

$29 \%^{b}$

$85 \%{ }^{c}$

a $2.5 \mathrm{~mol} \%[\mathrm{Rh}(\mathrm{OH})(\mathrm{cod})]_{2} \cdot{ }^{\mathrm{b}} 1$ equiv of $\mathrm{Et}{ }_{3} \mathrm{~N}$ and $0.5 \mathrm{M}$ in THF. ${ }^{\mathrm{c}} 1$ equiv of $\mathrm{Et} \mathrm{t}_{3} \mathrm{~N}$ and 3.3 equiv "Ar-B".

Table S8. Background reaction for substrate 1p<smiles></smiles>

Entry

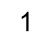

2

$3^{c}$

4

5

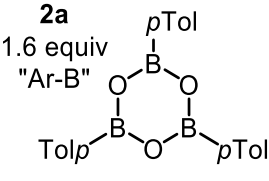

Catalyst

None

None

None

$[\mathrm{Rh}(\mathrm{OH})(\mathrm{cod})]_{2}{ }^{\mathrm{d}}$

$[\mathrm{Rh}(\mathrm{Cl})(\operatorname{cod})]_{2}{ }^{d}$
Orqanoboron

None

Present

Present

None

None

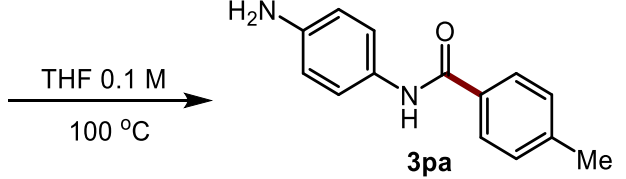

$\underline{\text { SM }}$ Product $^{\mathrm{a}}$

$30 \mathrm{~h}$

Present $^{b} \quad$ N.D.

Present $^{\mathrm{b}} \quad$ N.D.

Present $^{b} \quad$ N.D.

$0 \% \quad$ N.D.

$0 \% \quad$ N.D

${ }^{a} \mathrm{~N} . \mathrm{D} .=$ Not detected. ${ }^{\mathrm{b}} \mathrm{NMR}$ did not allow for quantitative analysis due to peak overlap. ${ }^{\mathrm{c}}$ Reaction run at $50{ }^{\circ} \mathrm{C}$ with 1 equiv of $\mathrm{Et}_{3} \mathrm{~N} .{ }^{\mathrm{d}} 5 \mathrm{~mol} \%[\mathrm{Rh}]_{2}$ 
Table S9. Effect of catalyst/organoboron loadings for the reaction of substrate 1p<smiles>Nc1ccc(NC(=O)c2ccccc2)cc1</smiles>

1

2

3

4

5

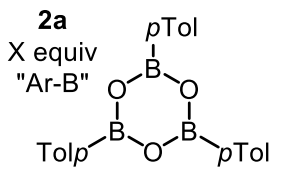

Catalyst

$5 \mathrm{~mol} \%$

$2.5 \mathrm{~mol} \%$

$1 \mathrm{~mol} \%$

$5 \mathrm{~mol} \%$

$2.5 \mathrm{~mol} \%$

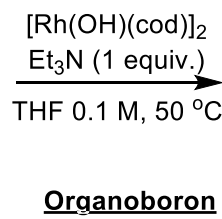

3.3 equiv.

3.3 equiv.

3.3 equiv.

1.6 equiv.

2.4 equiv.<smiles>Cc1ccc(C(=O)Nc2ccc(N)cc2)cc1</smiles>

Product

$92 \%$

$85 \%$

$35 \%^{\mathrm{a}}$

$22 \%^{a}$

$36 \%^{\mathrm{a}}$

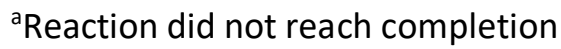

Table S10. Investigation of alternative organoboron reagents

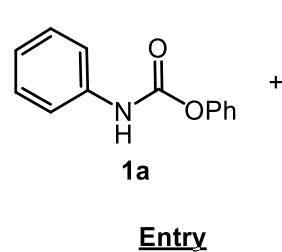

1

2

3<smiles>[R]OB([R9])c1ccccc1</smiles>

1.5 equiv.

Orqanoboron<smiles>C[Ge]1OC(C)(C)C(C)(C)O1</smiles><smiles></smiles>

$\mathrm{KF}_{3} \mathrm{~B}$ -
$[\mathrm{Rh}(\mathrm{OH})(\mathrm{cod})]_{2}$
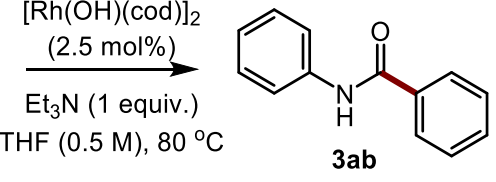

Yield

$<5 \%$

$53 \%$

$<5 \%$

\section{6-Synthesis of Amides from Table 2a-b}

General procedure $\mathbf{C}$ (low [Rh], low [RBO $]_{3}$, high [THF]): An oven-dried microwave vial equipped with a stir bar was cooled under a stream of argon followed by the addition of $[\mathrm{Rh}(\mathrm{OH})(\mathrm{cod})]_{2}\left(6.00 \times 10^{3} \mathrm{mmol}\right.$, 0.0100 equiv.), organoboron reagent (1.6 equiv "Ar-B"), and THF ( $1.20 \mathrm{~mL})$. Argon was bubbled through the solution for 2 minutes upon which the blocked isocyanate $\left(0.600 \mathrm{mmol}, 1.00\right.$ equiv.) and $\mathrm{Et}_{3} \mathrm{~N}(0.600$ mmol, 1.00 equiv.) were added. The microwave vial was sealed, purged with argon, and placed in a $50{ }^{\circ} \mathrm{C}$ oil bath. The reaction was monitored for the disappearance of starting material. Upon completion, the reaction was concentrated under reduced pressure, diluted with EtOAc $(40 \mathrm{~mL})$ and extracted $1 \mathrm{M} \mathrm{NaOH}$ $(1 \times 30 \mathrm{~mL})$, saturated $\mathrm{NaHCO}_{3}(1 \times 30 \mathrm{~mL})$, and brine $(1 \times 30 \mathrm{~mL})$. The organic layer was collected, dried 
over $\mathrm{Na}_{2} \mathrm{SO}_{4}$, and concentrated under reduced pressure. The crude mixture was purified by silica gel column chromatography providing the desired product.

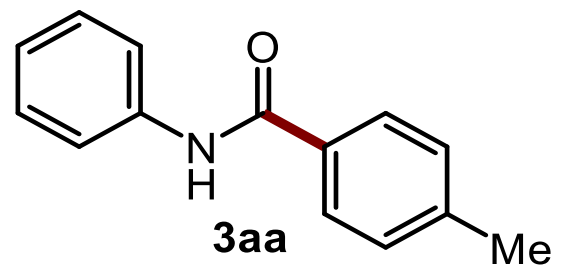

4-methyl- $\boldsymbol{N}$-phenylbenzamide (3aa): Synthesized according to general procedure $\mathbf{C}$ using masked isocyanate $1 \mathrm{a}(0.128 \mathrm{~g}, 0.600 \mathrm{mmol})$, organoboron reagent ${ }^{12} 2 \mathrm{a}(0.122 \mathrm{~g}, 0.976 \mathrm{mmol}, 1.6$ equiv "Ar-B"), $\mathrm{Et}_{3} \mathrm{~N}(0.084 \mathrm{~mL}, 0.600 \mathrm{mmol})$, and $[\mathrm{Rh}(\mathrm{OH})(\mathrm{cod})]_{2}\left(2.73 \mathrm{mg}, 6.00 \times 10^{3} \mathrm{mmol}\right)$. The reaction reached completion within 8 hours. The desired product was purified by column chromatography $(99 \%$ $\mathrm{CH}_{2} \mathrm{Cl}_{2} / \mathrm{Et}_{3} \mathrm{~N}$ ) yielding an amorphous white solid (0.109 g, $86 \%$ yield). $\mathrm{TLC} \mathrm{R}_{\mathrm{F}}=0.42$ in $99 \% \mathrm{CH}_{2} \mathrm{Cl}_{2} / \mathrm{Et}_{3} \mathrm{~N}$. ${ }^{1} \mathrm{H}-\mathrm{NMR}\left(400 \mathrm{MHz}, \mathrm{DMSO}-d_{6}\right): \delta 2.34(3 \mathrm{H}, \mathrm{s}), 7.06(1 \mathrm{H}, \mathrm{t}, J=7.4 \mathrm{~Hz}), 7.27-7.34(3 \mathrm{H}, \mathrm{m}), 7.79(1 \mathrm{H}, \mathrm{q}, J=7.5$ $\mathrm{Hz}), 7.87(1 \mathrm{H}, \mathrm{d}, J=8.2 \mathrm{~Hz}), 10.15(1 \mathrm{H}, \mathrm{s}) .{ }^{13} \mathrm{C}-\mathrm{NMR}\left(100 \mathrm{MHz}\right.$, DMSO- $\left.d_{6}\right): \delta 21.4\left(\mathrm{CH}_{3}\right), 120.8(\mathrm{CH}), 123.9$ $(\mathrm{CH}), 128.1(\mathrm{CH}), 129.0(\mathrm{CH}), 129.3(\mathrm{CH}), 132.6(\mathrm{C}), 139.7(\mathrm{C}), 142.0(\mathrm{C}), 165.8(\mathrm{C})$. Data is consistent with literature. ${ }^{13}$

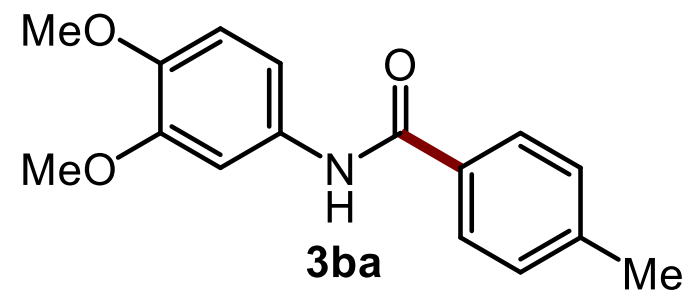

$\mathbf{N}$-(3,4-dimethoxyphenyl)-4-methylbenzamide (3ba): Synthesized according to general procedure $\mathbf{C}$ using masked isocyanate $1 \mathrm{~b}(0.164 \mathrm{~g}, 0.600 \mathrm{mmol})$, organoboron reagent $2 \mathrm{a}(0.122 \mathrm{~g}, 0.976 \mathrm{mmol}, 1.6$ equiv “Ar-B"), $E t_{3} \mathrm{~N}(0.084 \mathrm{~mL}, 0.600 \mathrm{mmol})$, and $[\mathrm{Rh}(\mathrm{OH})(\mathrm{cod})]_{2}\left(2.73 \mathrm{mg}, 6.00 \times 10^{3} \mathrm{mmol}\right)$. The reaction reached completion within 16 hours. The desired product was purified by column chromatography $195 \%$ $\mathrm{CH}_{2} \mathrm{Cl}_{2}$ /EtOAc) yielding an amorphous white solid (0.135 g, $83 \%$ yield). TLC $\mathrm{R}_{\mathrm{F}}=0.13$ in $\mathrm{CH}_{2} \mathrm{Cl}_{2} .{ }^{1} \mathrm{H}-\mathrm{NMR}$ $\left(400 \mathrm{MHz}, \mathrm{DMSO}-d_{6}\right): \delta 2.34(3 \mathrm{H}, \mathrm{s}), 3.70(3 \mathrm{H}, \mathrm{s}), 3.71(3 \mathrm{H}, \mathrm{s}), 6.88(2 \mathrm{H}, \mathrm{d}, \mathrm{J}=8.8 \mathrm{~Hz}), 7.27-7.31(3 \mathrm{H}, \mathrm{m})$, $7.44(1 \mathrm{H}, \mathrm{d}, J=2.4 \mathrm{~Hz}), 7.83(2 \mathrm{H}, \mathrm{d}, J=8.2 \mathrm{~Hz}), 9.98(1 \mathrm{H}, \mathrm{s}) .{ }^{13} \mathrm{C}-\mathrm{NMR}\left(100 \mathrm{MHz}, \mathrm{DMSO}-d_{6}\right): \delta 21.5\left(\mathrm{CH}_{3}\right)$, $55.8\left(\mathrm{CH}_{3}\right), 56.2\left(\mathrm{CH}_{3}\right), 105.9(\mathrm{CH}), 112.3(\mathrm{CH}), 112.7(\mathrm{CH}), 128.0(\mathrm{CH}), 129.3(\mathrm{CH}), 132.6(\mathrm{C}), 133.3(\mathrm{C})$, 141.8 (C), 145.5 (C), 148.9 (C), 165.4 (C). IR (film): 3307, 1642, $1231 \mathrm{~cm}^{-1}$. HRMS (EI): Exact mass calcd for $\mathrm{C}_{16} \mathrm{H}_{17} \mathrm{NO}_{3}[\mathrm{M}]^{+}:$271.1208. Found: 271.1189. 
<smiles>COC(=O)c1cc(NC(=O)c2ccc(C)cc2)cc(C(=O)OC)c1</smiles>

dimethyl 5-(4-methylbenzamido)isophthalate (3ca): Synthesized according to general procedure $\mathbf{C}$ using masked isocyanate $1 \mathrm{c}(0.198 \mathrm{~g}, 0.600 \mathrm{mmol})$, organoboron reagent $2 \mathrm{a}(0.122 \mathrm{~g}, 0.976 \mathrm{mmol}, 1.6$ equiv “Ar-B"), $\mathrm{Et}_{3} \mathrm{~N}(0.084 \mathrm{~mL}, 0.600 \mathrm{mmol})$, and $[\mathrm{Rh}(\mathrm{OH})(\mathrm{cod})]_{2}\left(2.73 \mathrm{mg}, 6.00 \times 10^{3} \mathrm{mmol}\right)$. The reaction reached completion within 16 hours. The desired product was purified by column chromatography $\left(\mathrm{CH}_{2} \mathrm{Cl}_{2}\right)$ yielding an amorphous white solid $\left(0.157 \mathrm{~g}, 80 \%\right.$ yield). TLC $\mathrm{R}_{\mathrm{F}}=0.30$ in $\mathrm{CH}_{2} \mathrm{Cl}_{2} .{ }^{1} \mathrm{H}-\mathrm{NMR}$ (400 $\left.\mathrm{MHz}, \mathrm{CDCl}_{3}\right): \delta 2.35(3 \mathrm{H}, \mathrm{s}), 3.84(6 \mathrm{H}, \mathrm{s}), 7.20(2 \mathrm{H}, \mathrm{d}, J=7.9 \mathrm{~Hz}), 7.77(2 \mathrm{H}, \mathrm{d}, \mathrm{J}=8.2 \mathrm{~Hz}), 8.35(1 \mathrm{H}, \mathrm{t}, J=1.5$ $\mathrm{Hz}), 8.50(2 \mathrm{H}, \mathrm{d}, J=1.5 \mathrm{~Hz}), 8.55(1 \mathrm{H}, \mathrm{s}, \mathrm{br}) .{ }^{13} \mathrm{C}-\mathrm{NMR}\left(100 \mathrm{MHz}\right.$, Acetone- $\left.d_{6}\right): \delta 20.6\left(\mathrm{CH}_{3}\right), 51.9\left(\mathrm{CH}_{3}\right)$, 124.6 (CH), 124.7 (CH), 124.9 (CH), 127.7 (CH), $129.1(\mathrm{CH}), 131.2(\mathrm{C}), 140.4(\mathrm{C}), 142.4(\mathrm{C}), 165.5(\mathrm{C}), 165.6$ (C). IR (film): $3275,1720,1236 \mathrm{~cm}^{-1}$. HRMS (EI): Exact mass calcd for $\mathrm{C}_{18} \mathrm{H}_{17} \mathrm{NO}_{5}[\mathrm{M}]^{+}:$327.1107. Found: 327.1113 .<smiles>CC(=O)c1cccc(NC(=O)c2ccc(C)cc2)c1</smiles>

$\mathbf{N}$-(3-acetylphenyl)-4-methylbenzamide (3da): Synthesized according to general procedure $\mathbf{C}$ using masked isocyanate $1 \mathbf{d}(0.153 \mathrm{~g}, 0.600 \mathrm{mmol})$, organoboron reagent $2 \mathrm{a}(0.122 \mathrm{~g}, 0.976 \mathrm{mmol}, 1.6$ equiv "Ar-B"), Et 3 N (0.084 mL, $0.600 \mathrm{mmol})$, and $[\mathrm{Rh}(\mathrm{OH})(\mathrm{cod})]_{2}(6.84 \mathrm{mg}, 0.0150 \mathrm{mmol})$. The reaction reached completion within 6 hours (note: yield was significantly reduced when reaction was left overnight). The desired product was purified by column chromatography $\left(90 \% \mathrm{CH}_{2} \mathrm{Cl}_{2} / \mathrm{EtOAC}\right)$ yielding an amorphous white solid ( $0.126 \mathrm{~g}, 80 \%$ yield). TLC $\mathrm{R}_{\mathrm{F}}=0.18 \mathrm{in} 90 \% \mathrm{CH}_{2} \mathrm{Cl}_{2} / \mathrm{EtOAc} .{ }^{1} \mathrm{H}-\mathrm{NMR}$ (400 MHz, DMSO- $\left.d_{6}\right): \delta$ $2.37(3 \mathrm{H}, \mathrm{s}), 2.57(3 \mathrm{H}, \mathrm{s}), 7.33(2 \mathrm{H}, \mathrm{d}, J=7.9 \mathrm{~Hz}), 7.49(1 \mathrm{H}, \mathrm{t}, J=7.9 \mathrm{~Hz}), 7.69(1 \mathrm{H}, \mathrm{d}, J=7.5 \mathrm{~Hz}), 7.90(2 \mathrm{H}, \mathrm{d}$, $J=7.9 \mathrm{~Hz}), 8.08(1 \mathrm{H}, \mathrm{d}, J=7.9 \mathrm{~Hz}), 8.38(1 \mathrm{H}, \mathrm{s}), 10.35(1 \mathrm{H}, \mathrm{s}) .{ }^{13} \mathrm{C}-\mathrm{NMR}\left(100 \mathrm{MHz}, \mathrm{DMSO}-d_{6}\right): \delta 21.5\left(\mathrm{CH}_{3}\right)$, $26.6\left(\mathrm{CH}_{3}\right), 120.0(\mathrm{CH}), 124.2(\mathrm{CH}), 125.2(\mathrm{CH}), 127.4(\mathrm{CH}), 129.3(\mathrm{CH}), 129.3(\mathrm{CH}), 131.7(\mathrm{C}), 137.6(\mathrm{C})$, 139.0 (C), 142.5 (C), 166.4 (C), 198.5 (C). IR (film): 3333, 1667, $1304 \mathrm{~cm}^{-1}$. HRMS (EI): Exact mass calcd for $\mathrm{C}_{16} \mathrm{H}_{15} \mathrm{NO}_{2}[\mathrm{M}]^{+}:$253.1103. Found: 253.1119. 


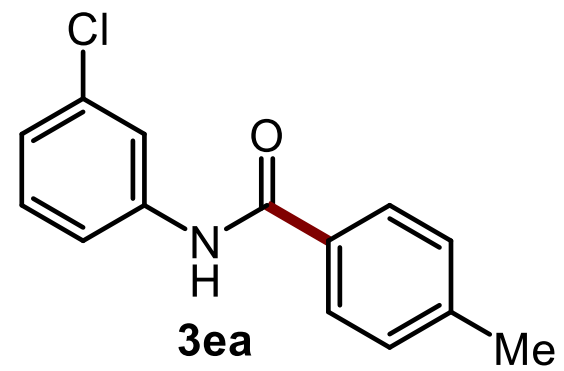

$\mathbf{N}$-(3-chlorophenyl)-4-methylbenzamide (3ea): Synthesized according to general procedure $\mathbf{C}$ using masked isocyanate $1 \mathrm{e}(0.149 \mathrm{~g}, 0.600 \mathrm{mmol})$, organoboron reagent $2 \mathrm{a}(0.122 \mathrm{~g}, 0.976 \mathrm{mmol}, 1.6$ equiv "Ar-B"), $\mathrm{Et}_{3} \mathrm{~N}(0.0836 \mathrm{~mL}, 0.600 \mathrm{mmol})$, and $[\mathrm{Rh}(\mathrm{OH})(\mathrm{cod})]_{2}(6.84 \mathrm{mg}, 0.0150 \mathrm{mmol})$. The reaction reached completion within 13 hours. The desired product was purified by column chromatography (75\% $\mathrm{CH}_{2} \mathrm{Cl}_{2}$ / hexanes) yielding an amorphous white solid ( $0.125 \mathrm{~g}$, 85\% yield). TLC $\mathrm{R}_{\mathrm{F}}=0.69 \mathrm{CH}_{2} \mathrm{Cl}_{2} .{ }^{1} \mathrm{H}-\mathrm{NMR}$ $\left(400 \mathrm{MHz}, \mathrm{DMSO}-d_{6}\right): \delta 2.35(3 \mathrm{H}, \mathrm{s}), 7.09-7.12(1 \mathrm{H}, \mathrm{m}), 7.29-7.35(3 \mathrm{H}, \mathrm{m}), 7.67-7.70(1 \mathrm{H}, \mathrm{m}), 7.84(2 \mathrm{H}, \mathrm{d}$, $J=8.2 \mathrm{~Hz}), 7.95(1 \mathrm{H}, \mathrm{t}, J=2.0 \mathrm{~Hz}), 10.28(1 \mathrm{H}, \mathrm{s}) .{ }^{13} \mathrm{C}-\mathrm{NMR}\left(100 \mathrm{MHz}, \mathrm{DMSO}-d_{6}\right): \delta 21.5\left(\mathrm{CH}_{3}\right), 119.1(\mathrm{CH})$, $120.2(\mathrm{CH}), 123.7(\mathrm{CH}), 128.2(\mathrm{CH}), 129.4(\mathrm{CH}), 130.7(\mathrm{CH}), 132.1(\mathrm{C}), 133.4(\mathrm{C}), 141.2(\mathrm{C}), 142.4(\mathrm{C}), 166.1$ (C). Data is consistent with literature. ${ }^{14}$<smiles>Cc1ccc(C(=O)Nc2ccc(Br)cc2)cc1</smiles>

$\mathbf{N}$-(4-bromophenyl)-4-methylbenzamide (3fa): Synthesized according to general procedure $\mathbf{C}$ using masked isocyanate $1 \mathrm{f}(0.175 \mathrm{~g}, 0.600 \mathrm{mmol})$, organoboron reagent $2 \mathrm{a}(0.122 \mathrm{~g}, 0.976 \mathrm{mmol}, 1.6$ equiv "Ar-B"), $E t_{3} \mathrm{~N}(0.0836 \mathrm{~mL}, 0.600 \mathrm{mmol})$, and $[\mathrm{Rh}(\mathrm{OH})(\mathrm{cod})]_{2}(6.84 \mathrm{mg}, 0.0150 \mathrm{mmol})$. The reaction reached completion within 13 hours. The desired product was purified by column chromatography (70\% $\mathrm{CH}_{2} \mathrm{Cl}_{2}$ / hexanes) yielding an amorphous white solid (0.124 g, $71 \%$ yield). $\mathrm{TLC} \mathrm{R}_{\mathrm{F}}=0.69$ in $\mathrm{CH}_{2} \mathrm{Cl}_{2} .{ }^{1} \mathrm{H}$ NMR $\left(400 \mathrm{MHz}, \mathrm{DMSO}-d_{6}\right): \delta 2.34(3 \mathrm{H}, \mathrm{s}), 7.30(2 \mathrm{H}, \mathrm{d}, J=8.0 \mathrm{~Hz}), 7.49(2 \mathrm{H}, \mathrm{d}, J=8.9 \mathrm{~Hz}), 7.74(2 \mathrm{H}, \mathrm{d}, J=8.9$ $\mathrm{Hz}), 7.83(2 \mathrm{H}, \mathrm{d}, J=8.2 \mathrm{~Hz}), 10.24(1 \mathrm{H}, \mathrm{s}) .{ }^{13} \mathrm{C}-\mathrm{NMR}\left(100 \mathrm{MHz}, \mathrm{DMSO}-d_{6}\right): \delta 21.5\left(\mathrm{CH}_{3}\right), 115.7(\mathrm{C}), 122.7$ $(\mathrm{CH}), 128.2(\mathrm{CH}), 129.4(\mathrm{CH}), 131.9(\mathrm{CH}), 132.3(\mathrm{C}), 139.1(\mathrm{C}), 142.2(\mathrm{C}), 165.9(\mathrm{C})$. Data is consistent with literature. ${ }^{13}$<smiles>Cc1ccc(C(=O)Nc2c(C)cc(C)cc2C)cc1</smiles>

$\mathbf{N}$-mesityl-4-methylbenzamide (3ga): Synthesized according to general procedure $\mathbf{C}$ using masked isocyanate $1 \mathrm{~g}(0.153 \mathrm{~g}, 0.600 \mathrm{mmol})$, organoboron reagent $2 \mathrm{a}(0.122 \mathrm{~g}, 0.976 \mathrm{mmol}, 1.6$ equiv “Ar-B"), 
$\mathrm{Et}_{3} \mathrm{~N}(0.0836 \mathrm{~mL}, 0.600 \mathrm{mmol})$, and $[\mathrm{Rh}(\mathrm{OH})(\mathrm{cod})]_{2}(6.84 \mathrm{mg}, 0.0150 \mathrm{mmol})$. The reaction reached completion within 16 hours. The desired product was purified by column chromatography $\left(\mathrm{CH}_{2} \mathrm{Cl}_{2}\right.$ to $5 \%$ EtOAC $/ \mathrm{CH}_{2} \mathrm{Cl}_{2}$ ) yielding an amorphous white solid (125.7 g, 83\% yield). TLC $\mathrm{R}_{\mathrm{F}}=0.16$ in $\mathrm{CH}_{2} \mathrm{Cl}_{2} .{ }^{1} \mathrm{H}-\mathrm{NMR}$ $\left(300 \mathrm{MHz}, \mathrm{DMSO}-d_{6}\right): \delta 2.13(6 \mathrm{H}, \mathrm{s}), 2.25(3 \mathrm{H}, \mathrm{s}), 2.38(3 \mathrm{H}, \mathrm{s}), 6.92(2 \mathrm{H}, \mathrm{s}), 7.31(2 \mathrm{H}, \mathrm{d}, J=8.0 \mathrm{~Hz}), 7.91$ $(2 \mathrm{H}, \mathrm{d}, \mathrm{J}=8.2 \mathrm{~Hz}), 9.59(1 \mathrm{H}, \mathrm{s}) .{ }^{13} \mathrm{C}-\mathrm{NMR}\left(75 \mathrm{MHz}, \mathrm{DMSO}-d_{6}\right): \delta 18.4\left(\mathrm{CH}_{3}\right), 21.0\left(\mathrm{CH}_{3}\right), 21.4\left(\mathrm{CH}_{3}\right), 127.9$ (CH), $128.7(\mathrm{CH}), 129.3(\mathrm{CH}), 132.1$ (C), 133.2 (C), 135.7 (C), 135.9 (C), 141.7 (C), 165.4 (C). IR (film): 3278, $3252,1652,1321 \mathrm{~cm}^{-1}$. HRMS (EI): Exact mass calcd for $\mathrm{C}_{17} \mathrm{H}_{19} \mathrm{NO}[\mathrm{M}]^{+}: 253.1467$. Found: 253.1446 .

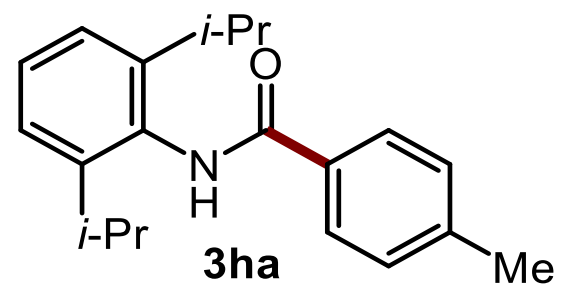

$\mathbf{N}$-(2,6-diisopropylphenyl)-4-methylbenzamide (3ha): Synthesized according to general procedure $\mathbf{C}$ using masked isocyanate $1 \mathrm{~h}(0.178 \mathrm{~g}, 0.600 \mathrm{mmol})$, organoboron reagent $2 \mathrm{a}(0.122 \mathrm{~g}, 0.976 \mathrm{mmol}, 1.6$ equiv "Ar-B"), $\mathrm{Et}_{3} \mathrm{~N}(0.0836 \mathrm{~mL}, 0.600 \mathrm{mmol})$, and $[\mathrm{Rh}(\mathrm{OH})(\mathrm{cod})]_{2}(6.84 \mathrm{mg}, 0.0150 \mathrm{mmol})$. The reaction reached completion within 16 hours. The desired product was purified by column chromatography (20\% EtOAc/ hexanes) yielding an amorphous white solid (0.104 g, 59\% yield). TLC $R_{F}=0.27$ in $15 \%$ EtOAc/ hexanes. ${ }^{1} \mathrm{H}-\mathrm{NMR}\left(300 \mathrm{MHz}, \mathrm{CDCl}_{3}\right): \delta 1.18(12 \mathrm{H}, \mathrm{d}, \mathrm{J}=6.9 \mathrm{~Hz}), 2.42(3 \mathrm{H}, \mathrm{s}), 3.13(2 \mathrm{H}, \mathrm{m}, \mathrm{J}=6.9 \mathrm{~Hz}), 7.21$ $(2 \mathrm{H}, \mathrm{d}, J=7.7 \mathrm{~Hz}), 7.34(1 \mathrm{H}, \mathrm{t}, J=7.7 \mathrm{~Hz}), 7.58(1 \mathrm{H}, \mathrm{s}), 7.77(2 \mathrm{H}, \mathrm{d}, J=8.2 \mathrm{~Hz}) .{ }^{13} \mathrm{C}-\mathrm{NMR}\left(100 \mathrm{MHz}, \mathrm{CDCl}_{3}\right): \delta$ $21.5\left(\mathrm{CH}_{3}\right), 23.7\left(\mathrm{CH}_{3}\right), 28.9(\mathrm{CH}), 123.5(\mathrm{CH}), 127.3(\mathrm{CH}), 128.4(\mathrm{CH}), 129.4(\mathrm{CH}), 131.5(\mathrm{C}), 131.6$ (C), 142.1 (C), 146.5 (C), 166.9 (C). Data is consistent with literature. ${ }^{15}$

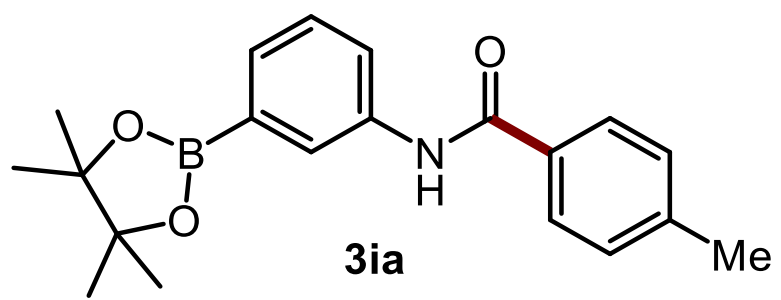

4-methyl- $N$-(3-(4,4,5,5-tetramethyl-1,3,2-dioxaborolan-2-yl)phenyl)benzamide (3ia): Synthesized according to general procedure $\mathbf{C}$ using masked isocyanate $1 \mathbf{i}(0.204 \mathrm{~g}, 0.600 \mathrm{mmol})$, organoboron reagent $2 \mathrm{a}\left(0.122 \mathrm{~g}, 0.976 \mathrm{mmol}, 1.6\right.$ equiv “Ar- $\left.\mathrm{B}^{\prime \prime}\right), \mathrm{Et}_{3} \mathrm{~N}(0.0836 \mathrm{~mL}, 0.600 \mathrm{mmol})$, and $[\mathrm{Rh}(\mathrm{OH})(\mathrm{cod})]_{2}$ $(6.84 \mathrm{mg}, 0.0150 \mathrm{mmol})$. The reaction reached completion within 6 hours. The desired product was extracted pure from the reaction mixture yielding an amorphous white solid $(0.0808 \mathrm{~g}, 40 \%$ yield). TLC $\mathrm{R}_{\mathrm{F}}=0.27$ in 20\% EtOAc/ hexanes. ${ }^{1} \mathrm{H}-\mathrm{NMR}\left(300 \mathrm{MHz}\right.$, DMSO- $\left.d_{6}\right): \delta 1.31(12 \mathrm{H}, \mathrm{s}), 2.36(3, \mathrm{~s}), 7.20(2 \mathrm{H}, \mathrm{d}$, $J=8.0 \mathrm{~Hz}), 7.34(1 \mathrm{H}, \mathrm{t}, J=7.7 \mathrm{~Hz}), 7.55(1 \mathrm{H}, \mathrm{d}, J=7.3 \mathrm{~Hz}), 7.72(2 \mathrm{H}, \mathrm{d}, J=8.1 \mathrm{~Hz}), 7.78(1 \mathrm{H}, \mathrm{s}), 8.01(2 \mathrm{H}, \mathrm{m})$. ${ }^{13} \mathrm{C}-\mathrm{NMR}\left(100 \mathrm{MHz}, \mathrm{CDCl}_{3}\right): \delta 21.5\left(\mathrm{CH}_{3}\right), 24.9\left(\mathrm{CH}_{3}\right), 83.9(\mathrm{C}), 123.4(\mathrm{CH}), 126.2(\mathrm{CH}), 127.1(\mathrm{CH}), 128.6$ (CH), 129.4 (CH), 130.7 (CH), 132.1 (C), 137.7 (C), 142.3 (C), 165.7 (C). IR (film): 3230, 1646, $1352 \mathrm{~cm}^{-1}$. HRMS (EI): Exact mass calcd for $\mathrm{C}_{20} \mathrm{H}_{24} \mathrm{BNO}_{3}[\mathrm{M}]^{+}$: 337.1849. Found: 337.1863. 


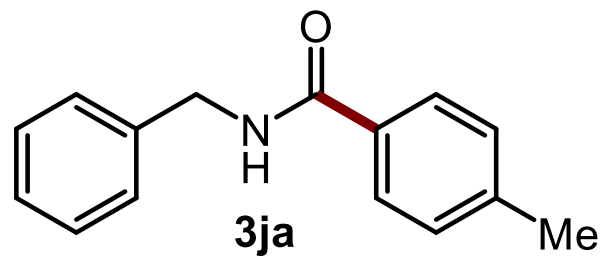

$\mathbf{N}$-benzyl-4-methylbenzamide (3ja): Synthesized according to general procedure $\mathbf{C}$ using masked isocyanate $1 \mathrm{j}$ ( $0.136 \mathrm{~g}, 0.600 \mathrm{mmol})$, organoboron reagent $2 \mathrm{a}(0.122 \mathrm{~g}, 0.976 \mathrm{mmol}, 1.6$ equiv "Ar-B"), $\mathrm{Et}_{3} \mathrm{~N}(0.0836 \mathrm{~mL}, 0.600 \mathrm{mmol})$, and $[\mathrm{Rh}(\mathrm{OH})(\mathrm{cod})]_{2}\left(2.73 \mathrm{mg}, 6.00 \times 10^{-3} \mathrm{mmol}\right)$. The reaction reached completion within 16 hours. The desired product was purified by column chromatography $\left(\mathrm{CH}_{2} \mathrm{Cl}_{2}\right)$ yielding an amorphous white solid $\left(0.107 \mathrm{~g}\right.$, 79\% yield). TLC $\mathrm{R}_{\mathrm{F}}=0.17$ in $\mathrm{CH}_{2} \mathrm{Cl}_{2} .{ }^{1} \mathrm{H}-\mathrm{NMR}(400 \mathrm{MHz}$, $\left.\mathrm{CDCl}_{3}\right): \delta 2.36(3 \mathrm{H}, \mathrm{s}), 4.59(2 \mathrm{H}, \mathrm{d}, J=5.7 \mathrm{~Hz}), 6.63(1 \mathrm{H}, \mathrm{s}, \mathrm{br}), 7.18(2 \mathrm{H}, \mathrm{d}, J=7.9 \mathrm{~Hz}), 7.24-7.34(5 \mathrm{H}, \mathrm{m})$, $7.67(2 \mathrm{H}, \mathrm{t}, J=4.1 \mathrm{~Hz}) .{ }^{13} \mathrm{C}-\mathrm{NMR}\left(100 \mathrm{MHz}, \mathrm{CDCl}_{3}\right): \delta 21.5\left(\mathrm{CH}_{3}\right), 44.0\left(\mathrm{CH}_{2}\right), 127.0(\mathrm{CH}), 127.5(\mathrm{CH}), 127.9$ $(\mathrm{CH}), 128.7(\mathrm{CH}), 129.2(\mathrm{CH}), 131.5(\mathrm{C}), 138.4(\mathrm{C}), 141.9(\mathrm{C}), 167.4(\mathrm{C})$. Data is consistent with literature. ${ }^{16}$<smiles>CNC(=O)c1ccc(C)cc1</smiles>

$\mathbf{N}$,4-dimethylbenzamide (3ka): Synthesized according to general procedure $\mathbf{C}$ using masked isocyanate $1 \mathrm{k}(0.0906 \mathrm{~g}, 0.600 \mathrm{mmol})$, organoboron reagent $2 \mathrm{a}\left(0.122 \mathrm{~g}, 0.976 \mathrm{mmol}, 1.6\right.$ equiv "Ar-B"), $\mathrm{Et}_{3} \mathrm{~N}$ $(0.0836 \mathrm{~mL}, 0.600 \mathrm{mmol})$, and $[\mathrm{Rh}(\mathrm{OH})(\mathrm{cod})]_{2}(6.84 \mathrm{mg}, 0.0150 \mathrm{mmol})$. The reaction reached completion within 14 hours. The desired product was purified by column chromatography (25\% EtOAc/ hexanes) yielding an amorphous white solid $\left(0.0651 \mathrm{~g}, 73 \%\right.$ yield). TLC $\mathrm{R}_{\mathrm{F}}=0.26$ in $25 \%$ EtOAc/ hexanes. ${ }^{1} \mathrm{H}-\mathrm{NMR}$ $\left(400 \mathrm{MHz}, \mathrm{CDCl}_{3}\right): \delta 2.32(3 \mathrm{H}, \mathrm{s}), 2.91(3 \mathrm{H}, \mathrm{d}, \mathrm{J}=4.7 \mathrm{~Hz}), 6.73(1 \mathrm{H}, \mathrm{s}, \mathrm{br}), 7.13(2 \mathrm{H}, \mathrm{d}, J=7.9 \mathrm{~Hz}), 7.64(2 \mathrm{H}, \mathrm{d}$, $J=8.0 \mathrm{~Hz}$ ). ${ }^{13} \mathrm{C}-\mathrm{NMR}\left(100 \mathrm{MHz}, \mathrm{CDCl}_{3}\right): \delta 21.4\left(\mathrm{CH}_{3}\right), 26.7\left(\mathrm{CH}_{3}\right), 126.9(\mathrm{CH}), 129.1(\mathrm{CH}), 131.7(\mathrm{C}), 141.6$ (C), 168.4 (C). Data is consistent with literature. ${ }^{17}$<smiles>COC(=O)C(NC(=O)c1ccc(C)cc1)c1ccccc1</smiles>

methyl 2-(4-methylbenzamido)-2-phenylacetate (3la): Synthesized according to general procedure C using masked isocyanate $1 \mathrm{l}(0.171 \mathrm{~g}, 0.600 \mathrm{mmol})$, organoboron reagent $2 \mathrm{a}(0.122 \mathrm{~g}, 0.976 \mathrm{mmol}, 1.6$ equiv "Ar-B"), $\mathrm{Et}_{3} \mathrm{~N}(0.0836 \mathrm{~mL}, 0.600 \mathrm{mmol})$, and $[\mathrm{Rh}(\mathrm{OH})(\mathrm{cod})]_{2}\left(2.73 \mathrm{mg}, 6.00 \times 10^{-3} \mathrm{mmol}\right)$. The reaction reached completion within 16 hours. The desired product was purified by column chromatography $\left(\mathrm{CH}_{2} \mathrm{Cl}_{2}\right)$ yielding an amorphous white solid $\left(0.131 \mathrm{~g}, 81 \%\right.$ yield). TLC $\mathrm{R}_{\mathrm{F}}=0.33$ in $\mathrm{CH}_{2} \mathrm{Cl}_{2}$. ${ }^{1} \mathrm{H}-\mathrm{NMR}(400 \mathrm{MHz}, \mathrm{CDCl})$ ): $\delta 2.35(3 \mathrm{H}, \mathrm{s}), 3.71(3 \mathrm{H}, \mathrm{s}), 5.76(1 \mathrm{H}, \mathrm{d}, J=7.1 \mathrm{~Hz}), 7.18(2 \mathrm{H}, \mathrm{d}, J=7.9 \mathrm{~Hz}), 7.24-$ $7.36(4 \mathrm{H}, \mathrm{m}), 7.40-7.44(2 \mathrm{H}, \mathrm{m}), 7.70(2 \mathrm{H}, \mathrm{d}, J=8.2 \mathrm{~Hz}) .{ }^{13} \mathrm{C}-\mathrm{NMR}\left(100 \mathrm{MHz}, \mathrm{CDCl}_{3}\right): \delta 21.5\left(\mathrm{CH}_{3}\right), 52.8$ 
$\left(\mathrm{CH}_{3}\right), 56.8(\mathrm{CH}), 127.2(\mathrm{CH}), 127.3(\mathrm{CH}), 128.6(\mathrm{CH}), 129.0(\mathrm{CH}), 129.2(\mathrm{CH}), 130.8(\mathrm{C}), 136.7(\mathrm{C}), 142.3$ (C), 166.6 (C), 171.6 (C). IR (film): 3348, 1738, 1637, 1322, $1175 \mathrm{~cm}^{-1}$. HRMS (EI): Exact mass calcd for $\mathrm{C}_{17} \mathrm{H}_{17} \mathrm{NO}_{3}[\mathrm{M}]^{+}:$283.1208. Found: 283.1223. Chiral HPLC: ChiralPak OD-H, $i-\mathrm{PrOH} /$ Hexane $=10: 90,1.0$ $\mathrm{mL} / \mathrm{min}, 230 \mathrm{~nm}, t=9.54 \mathrm{~min}$ and $12.89 \mathrm{~min}$

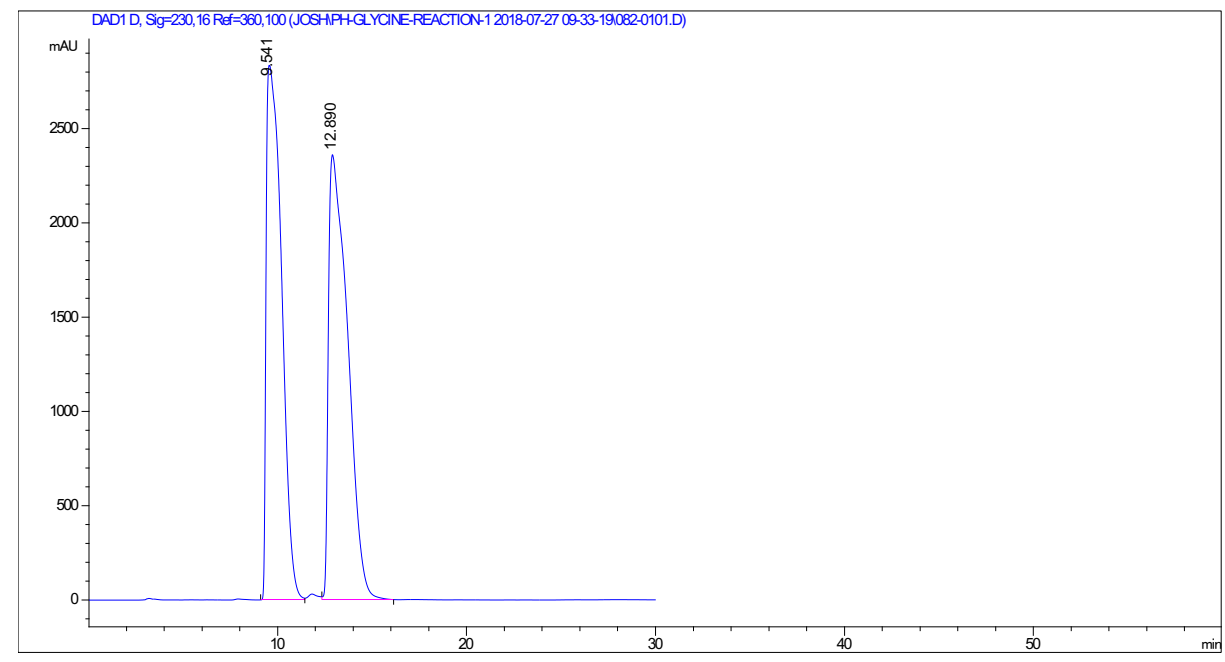

\# Time Area Height Width Area\% Symmetry

$\begin{array}{lllllll}1 & 9.541 & 160126.1 & 2836.6 & 0.7206 & 49.043 & 0.229\end{array}$

$\begin{array}{lllllll}2 & 12.89 & 166375.2 & 2361.9 & 0.9378 & 50.957 & 0.25\end{array}$

Figure S3. HPLC trace and data of 3la showing complete racemization

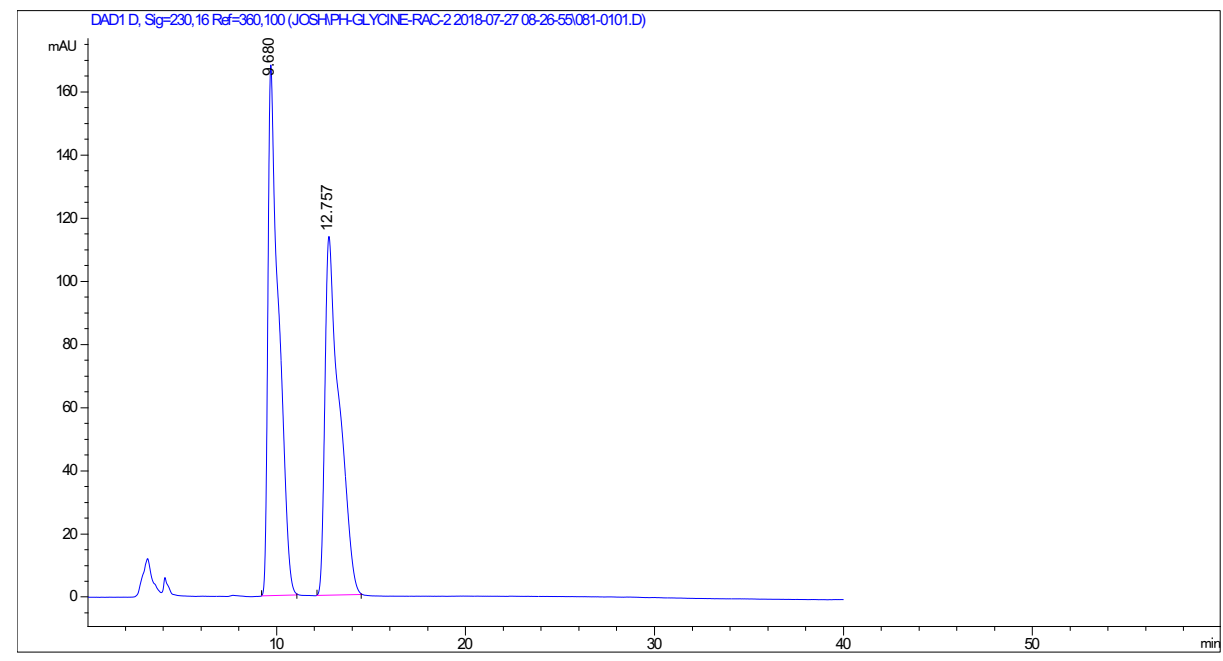

\# Time Area Height Width Area\% Symmetry

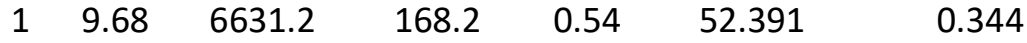

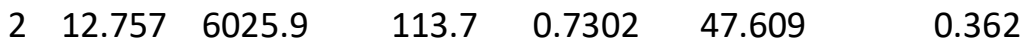

Figure S4. HPLC trace and data of racemic 3la 


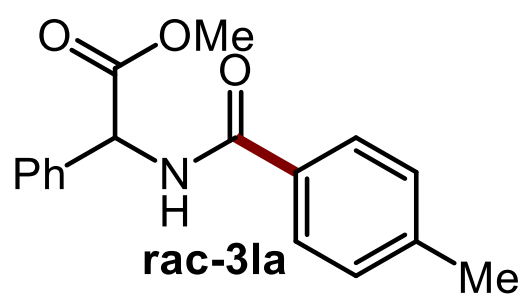

methyl 2-(4-methylbenzamido)-2-phenylacetate (rac-3la): Synthesized according to general procedure B using 2-phenylglycine methyl ester hydrochloride $(0.101 \mathrm{~g}, 0.500 \mathrm{mmol}), \mathrm{Et}_{3} \mathrm{~N}(0.168 \mathrm{~mL}, 1.20 \mathrm{mmol})$, and p-toluoyl chloride $(0.0730 \mathrm{~mL}, 0.550 \mathrm{mmol})$, and $\mathrm{CH}_{2} \mathrm{Cl}_{2}(1.60 \mathrm{~mL})$. The reaction reached completion within 1 hour. The desired product was purified by column chromatography $\left(100 \% \mathrm{CH}_{2} \mathrm{Cl}_{2}\right)$ yielding an amorphous white solid $(0.0831 \mathrm{~g}, 59 \%$ yield). Chiral HPLC: ChiralPak column OD-H $i-\mathrm{PrOH} / \mathrm{Hexane}=$ $10: 90,1.0 \mathrm{~mL} / \mathrm{min}, 230 \mathrm{~nm}, t=9.5 \mathrm{~min}, 12.9 \mathrm{~min}$.<smiles>COC(CNC(=O)c1ccc(C)cc1)OC</smiles>

$\mathbf{N}$-(2,2-dimethoxyethyl)-4-methylbenzamide (3ma): Synthesized according to general procedure $\mathbf{C}$ using masked isocyanate $1 \mathrm{~m}(0.135 \mathrm{~g}, 0.600 \mathrm{mmol})$, organoboron reagent $2 \mathrm{a}(0.122 \mathrm{~g}, 0.976 \mathrm{mmol}, 1.6$ equiv "Ar-B"), Et $\mathrm{H}_{3} \mathrm{~N}(0.0836 \mathrm{~mL}, 0.600 \mathrm{mmol})$, and $[\mathrm{Rh}(\mathrm{OH})(\mathrm{cod})]_{2}(6.84 \mathrm{mg}, 0.0150 \mathrm{mmol})$. The reaction reached completion within 5 hours. The desired product was purified by column chromatography ( $45 \%$ EtOAc/ hexanes) yielding an amorphous white solid ( $0.0977 \mathrm{~g}, 73 \%$ yield). TLC $\mathrm{R}_{\mathrm{F}}=0.31$ in $45 \% \mathrm{EtOAc} /$ hexanes. ${ }^{1} \mathrm{H}-\mathrm{NMR}\left(400 \mathrm{MHz}, \mathrm{CDCl}_{3}\right): \delta 2.32(3 \mathrm{H}, \mathrm{s}), 3.36(6 \mathrm{H}, \mathrm{s}), 3.53(2 \mathrm{H}, \mathrm{t}, J=5.6 \mathrm{~Hz}), 4.44(1 \mathrm{H}, \mathrm{t}, J=5.3$ $\mathrm{Hz}), 6.51(1 \mathrm{H}, \mathrm{s}, \mathrm{br}), 7.15(2 \mathrm{H}, \mathrm{d}, J=8.0 \mathrm{~Hz}), 7.63(2 \mathrm{H}, \mathrm{d}, J=8.1 \mathrm{~Hz}) .{ }^{13} \mathrm{C}-\mathrm{NMR}\left(100 \mathrm{MHz}, \mathrm{CDCl}_{3}\right): \delta 21.4\left(\mathrm{CH}_{3}\right)$, $41.5\left(\mathrm{CH}_{2}\right), 54.5\left(\mathrm{CH}_{3}\right), 102.8(\mathrm{CH}), 127.0(\mathrm{CH}), 129.1(\mathrm{CH}), 131.5(\mathrm{C}), 141.9$ (C), 167.5 (C). IR (film): 3264 , 1631, $1111 \mathrm{~cm}^{-1}$. HRMS (EI): Exact mass calcd for $\mathrm{C}_{12} \mathrm{H}_{17} \mathrm{NO}_{3} \mathrm{Na}[\mathrm{M}+\mathrm{Na}]^{+}: 246.1106$. Found: 246.1114 .

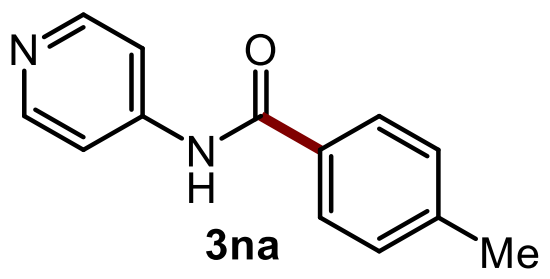

4-methyl-N-(pyridin-4-yl)benzamide (3na): Synthesized according to general procedure $\mathbf{D}$ using masked isocyanate $1 \mathrm{n}(0.128 \mathrm{~g}, 0.600 \mathrm{mmol})$, organoboron reagent $2 \mathrm{a}(0.408 \mathrm{~g}, 3.25 \mathrm{mmol}, 5.4$ equiv “Ar-B"), $\mathrm{Et}_{3} \mathrm{~N}(0.0836 \mathrm{~mL}, 0.600 \mathrm{mmol})$, and $[\mathrm{Rh}(\mathrm{OH})(\mathrm{cod})]_{2}(6.84 \mathrm{mg}, 0.0150 \mathrm{mmol})$ at $120^{\circ} \mathrm{C}$. The reaction reached completion within 2 hours. The desired product was purified by column chromatography ( $5 \%$ $\mathrm{MeOH} / \mathrm{EtOAC}$ ) yielding an amorphous white solid (0.0840 g, 66\% yield). TLC $\mathrm{R}_{\mathrm{F}}=0.19$ in $5 \%$ $\mathrm{MeOH} /$ EtOAc. ${ }^{1} \mathrm{H}-\mathrm{NMR}\left(300 \mathrm{MHz}, \mathrm{DMSO}-\mathrm{d}_{6}\right): \delta 2.38(3 \mathrm{H}, \mathrm{s}), 7.35(2 \mathrm{H}, \mathrm{d}, \mathrm{J}=8.0 \mathrm{~Hz}), 7.79(2 \mathrm{H}, \mathrm{q}, \mathrm{J}=6.3 \mathrm{~Hz})$, $7.89(2 \mathrm{H}, \mathrm{d}, J=8.1 \mathrm{~Hz}), 8.47(2 \mathrm{H}, \mathrm{d}, J=6.1 \mathrm{~Hz}), 10.50(1 \mathrm{H}, \mathrm{s}) .{ }^{13} \mathrm{C}-\mathrm{NMR}\left(75 \mathrm{MHz}, \mathrm{DMSO}-d_{6}\right): \delta 21.5\left(\mathrm{CH}_{3}\right)$, 
114.4 (CH), $128.3(\mathrm{CH}), 129.4(\mathrm{CH}), 131.8$ (C), 142.7 (C), 146.4 (C), 150.7 (CH), 166.7 (C). IR (film): 3337, 1651, $1332 \mathrm{~cm}^{-1}$. HRMS (EI): Exact mass calcd for $\mathrm{C}_{13} \mathrm{H}_{12} \mathrm{~N}_{2} \mathrm{O}[\mathrm{M}]^{+}:$212.0950. Found: 212.0957.

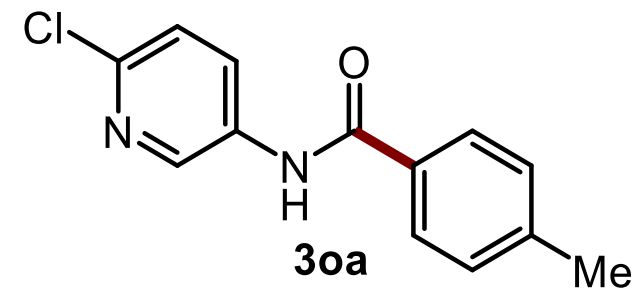

$\mathrm{N}$-(6-chloropyridin-3-yl)-4-methylbenzamide (30a): Synthesized according to general procedure D using masked isocyanate $10(0.149 \mathrm{~g}, 0.600 \mathrm{mmol})$, organoboron reagent $2 \mathrm{a}(0.245 \mathrm{~g}, 1.95 \mathrm{mmol}, 3.3$ equiv “Ar-B"), $\mathrm{Et}_{3} \mathrm{~N}(0.0836 \mathrm{~mL}, 0.600 \mathrm{mmol})$, and $[\mathrm{Rh}(\mathrm{OH})(\mathrm{cod})]_{2}(6.84 \mathrm{mg}, 0.0150 \mathrm{mmol})$. The reaction reached completion within 20 hours. The crude mixture was dry loaded onto silica gel $(\mathrm{MeOH})$ and purified by column chromatography ( $5 \%$ to $10 \% \mathrm{EtOAc} / \mathrm{CH}_{2} \mathrm{Cl}_{2}$ ) yielding and amorphous white solid (0.113 g, 76\% yield). TLC RF $=0.17$ in $5 \%$ EtOAc/ $\mathrm{CH}_{2} \mathrm{Cl}_{2} .{ }^{1} \mathrm{H}-\mathrm{NMR}\left(300 \mathrm{MHz}, \mathrm{DMSO}-d_{6}\right): \delta 2.38(3 \mathrm{H}, \mathrm{s}), 7.35$ $(2 \mathrm{H}, \mathrm{d}, J=8.0 \mathrm{~Hz}), 7.50(1 \mathrm{H}, \mathrm{d}, J=8.7 \mathrm{~Hz}), 7.88(2 \mathrm{H}, \mathrm{d}, J=8.1 \mathrm{~Hz}), 8.25(1 \mathrm{H}, \mathrm{dd}, J=2.8 \mathrm{~Hz}, 8.7 \mathrm{~Hz}), 8.79(1 \mathrm{H}, \mathrm{d}$, $J=2.5 \mathrm{~Hz}$ ), $10.48(1 \mathrm{H}, \mathrm{s}) \cdot{ }^{13} \mathrm{C}-\mathrm{NMR}\left(75 \mathrm{MHz}\right.$, DMSO- $\left.d_{6}\right): \delta 21.5\left(\mathrm{CH}_{3}\right), 124.5(\mathrm{CH}), 128.2(\mathrm{CH}), 129.4(\mathrm{CH})$, $131.23(\mathrm{CH}), 131.6(\mathrm{C}), 136.0$ (C), $141.9(\mathrm{CH}), 142.6$ (C), 144.3 (C), 166.1 (C). IR (film): 3332, 1652, 1289, $825 \mathrm{~cm}^{-1}$. HRMS (EI): Exact mass calcd for $\mathrm{C}_{13} \mathrm{H}_{11} \mathrm{~N}_{2} \mathrm{OCl}[\mathrm{M}]^{+}: 246.0560$. Found: 246.0564 .

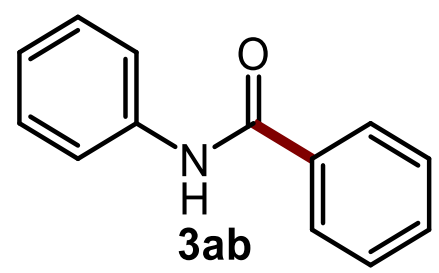

$\mathbf{N}$-phenylbenzamide (3ab): Synthesized according to general procedure $\mathbf{C}$ using masked isocyanate $\mathbf{1 a}$ $(0.128 \mathrm{~g}, 0.600 \mathrm{mmol})$, organoboron reagent $\mathbf{2 b}(0.110 \mathrm{~g}, 0.900 \mathrm{mmol}), \mathrm{Et}_{3} \mathrm{~N}(0.0836 \mathrm{~mL}, 0.600 \mathrm{mmol})$, and $[\mathrm{Rh}(\mathrm{OH})(\mathrm{cod})]_{2}\left(2.73 \mathrm{mg}, 6.00 \times 10^{-3} \mathrm{mmol}\right)$. The reaction reached completion within 16 hours. The desired product was purified by column chromatography $\left(\mathrm{CH}_{2} \mathrm{Cl}_{2}\right)$ yielding an amorphous white solid (0.0929 g, 81\% yield). TLC $R_{F}=0.40$ in $\mathrm{CH}_{2} \mathrm{Cl}_{2} .{ }^{1} \mathrm{H}-\mathrm{NMR}\left(400 \mathrm{MHz}, \mathrm{DMSO}-d_{6}\right): \delta 7.10(1 \mathrm{H}, \mathrm{t}, J=7.4 \mathrm{~Hz}), 7.35$ $(2 \mathrm{H}, \mathrm{t}, J=7.5 \mathrm{~Hz}), 7.50-7.60(3 \mathrm{H}, \mathrm{m}), 7.80(2 \mathrm{H}, \mathrm{d}, J=8.7 \mathrm{~Hz}), 7.95-7.98(2 \mathrm{H}, \mathrm{m}), 10.26(1 \mathrm{H}, \mathrm{s}) .{ }^{13} \mathrm{C}-\mathrm{NMR}(100$ MHz, DMSO- $\left.d_{6}\right): \delta 120.8(\mathrm{CH}), 124.1(\mathrm{CH}), 128.1(\mathrm{CH}), 128.8(\mathrm{CH}), 129.1(\mathrm{CH}), 132.0(\mathrm{CH}), 135.5(\mathrm{C}), 139.7$ (C), 166.0 (C). Data is consistent with literature. ${ }^{18}$<smiles>Cc1ccccc1C(=O)Nc1ccccc1</smiles> 
2-methyl- $\boldsymbol{N}$-phenylbenzamide (3ac): Synthesized according to general procedure $\mathbf{C}$ using masked isocyanate $1 \mathrm{a}(0.128 \mathrm{~g}, 0.600 \mathrm{mmol})$, boroxine $2 \mathrm{c}(0.107 \mathrm{~g}, 0.300 \mathrm{mmol}), \mathrm{Et}_{3} \mathrm{~N}(0.0836 \mathrm{~mL}, 0.600 \mathrm{mmol})$, and $[\mathrm{Rh}(\mathrm{OH})(\mathrm{cod})]_{2}(6.84 \mathrm{mg}, 0.0150 \mathrm{mmol})$. The reaction reached completion within 16 hours. The desired product was purified by column chromatography $\left(75 \% \mathrm{CH}_{2} \mathrm{Cl}_{2}\right.$ to $\left.100 \% \mathrm{CH}_{2} \mathrm{Cl}_{2}\right)$ yielding an amorphous white solid (0.109 g, 86\% yield). TLC $\mathrm{R}_{\mathrm{F}}=0.47$ in $\mathrm{CH}_{2} \mathrm{Cl}_{2} \cdot{ }^{1} \mathrm{H}-\mathrm{NMR}$ (400 MHz, DMSO- $\left.d_{6}\right): \delta 2.40$ $(3 \mathrm{H}, \mathrm{s}), 7.10(1 \mathrm{H}, \mathrm{t}, J=7.4 \mathrm{~Hz}), 7.28-7.42(5 \mathrm{H}, \mathrm{m}), 7.47(1 \mathrm{H}, \mathrm{d}, J=7.4 \mathrm{~Hz}), 7.78(2 \mathrm{H}, \mathrm{d}, J=7.8 \mathrm{~Hz}), 10.31(1 \mathrm{H}$, s). ${ }^{13} \mathrm{C}-\mathrm{NMR}\left(100 \mathrm{MHz}\right.$, DMSO-d $\left.d_{6}\right): \delta 19.8\left(\mathrm{CH}_{3}\right), 120.1(\mathrm{CH}), 124.0(\mathrm{CH}), 126.1(\mathrm{CH}), 127.7(\mathrm{CH}), 129.1$ $(\mathrm{CH}), 130.0(\mathrm{CH}), 131.0(\mathrm{CH}), 135.6(\mathrm{C}), 137.8(\mathrm{C}), 139.8(\mathrm{C}), 168.3(\mathrm{C})$. Data is consistent with literature. ${ }^{19}$

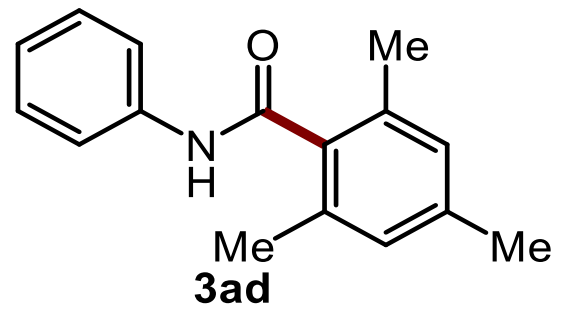

2,4,6-trimethyl- $\mathbf{N}$-phenylbenzamide (3ad): Synthesized according to general procedure $\mathbf{C}$ using masked isocyanate $1 \mathrm{a}(0.128 \mathrm{~g}, 0.600 \mathrm{mmol})$, organoboron reagent $2 \mathrm{~d}(0.263 \mathrm{~g}, 0.600 \mathrm{mmol}), \mathrm{Et}_{3} \mathrm{~N}(0.0836 \mathrm{~mL}$, $0.600 \mathrm{mmol})$, and $[\mathrm{Rh}(\mathrm{OH})(\mathrm{cod})]_{2}(6.84 \mathrm{mg}, 0.0150 \mathrm{mmol})$. The reaction reached completion within 20 hours. The desired product was purified by column chromatography $\left(\mathrm{CH}_{2} \mathrm{Cl}_{2}\right)$ yielding an amorphous white solid $\left(0.0960 \mathrm{~g}, 67 \%\right.$ yield). TLC $\mathrm{R}_{\mathrm{F}}=0.43$ in $\mathrm{CH}_{2} \mathrm{Cl}_{2} .{ }^{1} \mathrm{H}-\mathrm{NMR}\left(400 \mathrm{MHz}\right.$, DMSO- $\left.d_{6}\right)$ : $\delta$ 2.24-2.27 (9H, m), $6.92(2 \mathrm{H}, \mathrm{s}), 7.08(1 \mathrm{H}, \mathrm{t}, \mathrm{J}=7.4 \mathrm{~Hz}), 7.33(2 \mathrm{H}, \mathrm{t}, \mathrm{J}=8.3 \mathrm{~Hz}), 7.74(2 \mathrm{H}, \mathrm{d}, \mathrm{J}=7.6 \mathrm{~Hz}), 10.29(1 \mathrm{H}, \mathrm{s}) .{ }^{13} \mathrm{C}-\mathrm{NMR}$ (100 MHz, DMSO-d $\left.d_{6}\right): \delta 19.3\left(\mathrm{CH}_{3}\right), 21.1\left(\mathrm{CH}_{3}\right), 120.0(\mathrm{CH}), 123.9(\mathrm{CH}), 128.3(\mathrm{CH}), 129.2(\mathrm{CH}), 134.1(\mathrm{C})$, 136.3 (C), 138.1 (C), 139.7 (C), 168.6 (C). Data is consistent with literature. ${ }^{20}$

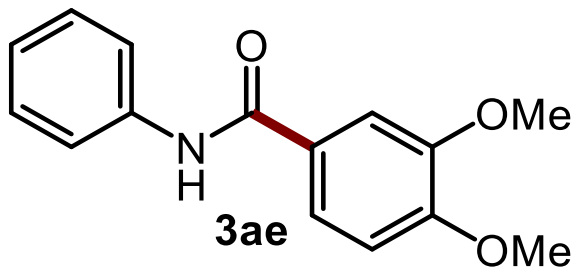

3,4-dimethoxy- $\boldsymbol{N}$-phenylbenzamide (3ae): Synthesized according to general procedure $\mathbf{C}$ using masked isocyanate 1a $(0.128 \mathrm{~g}, 0.600 \mathrm{mmol})$, boroxine $2 \mathrm{e}(0.148 \mathrm{~g}, 0.300 \mathrm{mmol}), \mathrm{Et}_{3} \mathrm{~N}(0.0836 \mathrm{~mL}, 0.600 \mathrm{mmol})$, and $[\mathrm{Rh}(\mathrm{OH})(\mathrm{cod})]_{2}\left(2.73 \mathrm{mg}, 6.00 \times 10^{-3} \mathrm{mmol}\right)$. The reaction reached completion within 8 hours. The desired product was purified by column chromatography $\left(95 \% \mathrm{CH}_{2} \mathrm{Cl}_{2} /\right.$ EtOAc) yielding an amorphous white solid (0.114 g, 74\% yield). TLC RF $=0.33$ in 95\% $\mathrm{CH}_{2} \mathrm{Cl}_{2} / \mathrm{EtOAc}^{1}{ }^{1} \mathrm{H}-\mathrm{NMR}$ (400 MHz, DMSO- $d_{6}$ ): $\delta$ 3.80-3.82 $(6 \mathrm{H}, \mathrm{m}), 7.03-7.07(2 \mathrm{H}, \mathrm{m}), 7.29-7.33(2 \mathrm{H}, \mathrm{m}), 7.51(1 \mathrm{H}, \mathrm{d}, J=2.1 \mathrm{~Hz}), 7.60(1 \mathrm{H}, \mathrm{dd}, J=2.1,8.4$ $\mathrm{Hz}), 7.71-7.74(2 \mathrm{H}, \mathrm{m}), 10.04(1 \mathrm{H}, \mathrm{s}) .{ }^{13} \mathrm{C}-\mathrm{NMR}\left(100 \mathrm{MHz}, \mathrm{DMSO}-d_{6}\right): \delta 56.1\left(\mathrm{CH}_{3}\right), 56.1\left(\mathrm{CH}_{3}\right), 111.4(\mathrm{CH})$, $111.5(\mathrm{CH}), 121.0(\mathrm{CH}), 121.5(\mathrm{CH}), 123.9(\mathrm{CH}), 127.5(\mathrm{C}), 129.0(\mathrm{CH}), 139.7(\mathrm{C}), 148.8(\mathrm{C}), 152.1(\mathrm{C}), 165.4$ (C). Data is consistent with literature. ${ }^{21}$ 


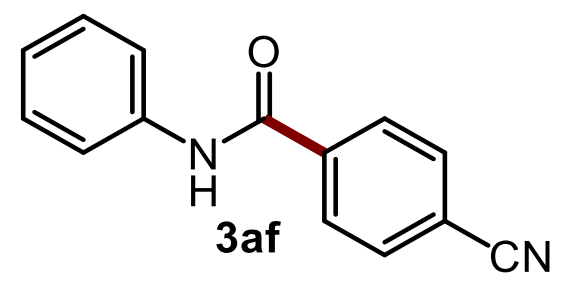

4-cyano- $\mathbf{N}$-phenylbenzamide (3af): Synthesized according to general procedure $\mathbf{C}$ using masked isocyanate $1 \mathrm{a}(0.128 \mathrm{~g}, 0.600 \mathrm{mmol})$, boroxine $2 \mathrm{f}(0.116 \mathrm{~g}, 0.300 \mathrm{mmol}), \mathrm{Et}_{3} \mathrm{~N}(0.0836 \mathrm{~mL}, 0.600 \mathrm{mmol})$, and $[\mathrm{Rh}(\mathrm{OH})(\mathrm{cod})]_{2}\left(2.73 \mathrm{mg}, 6.00 \times 10^{-3} \mathrm{mmol}\right)$. The reaction reached completion within 12 hours. The desired product was purified by column chromatography $\left(\mathrm{CH}_{2} \mathrm{Cl}_{2}\right.$ to $\left.96 \% \mathrm{CH}_{2} \mathrm{Cl}_{2} / \mathrm{EtOAc}\right)$ yielding an amorphous white solid (0.0925 g, 69\% yield). TLC $\mathrm{R}_{\mathrm{F}}=0.17$ in $\mathrm{CH}_{2} \mathrm{Cl}_{2} .{ }^{1} \mathrm{H}-\mathrm{NMR}\left(400 \mathrm{MHz}, \mathrm{DMSO}-d_{6}\right): \delta$ $7.14(1 \mathrm{H}, \mathrm{t}, J=7.4 \mathrm{~Hz}), 7.38(2 \mathrm{H}, \mathrm{t}, \mathrm{J}=8.5 \mathrm{~Hz}), 7.78(2 \mathrm{H}, \mathrm{d}, \mathrm{J}=8.7 \mathrm{~Hz}), 8.01-8.04(2 \mathrm{H}, \mathrm{m}), 8.10-8.12(2 \mathrm{H}, \mathrm{m})$, $10.48(1 \mathrm{H}, \mathrm{s}) .{ }^{13} \mathrm{C}-\mathrm{NMR}\left(100 \mathrm{MHz}\right.$, DMSO- $\left.d_{6}\right): \delta 114.3(\mathrm{C}), 118.8(\mathrm{C}), 120.9(\mathrm{CH}), 124.6(\mathrm{CH}), 129.0(\mathrm{CH})$, 129.2 (CH), 132.9 (CH), 139.2 (C), 139.5 (C), 164.6 (C). IR (film): 3353, 2232, $1653 \mathrm{~cm}^{-1}$. HRMS (EI): Exact mass calcd for $\mathrm{C}_{14} \mathrm{H}_{10} \mathrm{~N}_{2} \mathrm{O}[\mathrm{M}]^{+}:$222.0793. Found: 222.0799 .

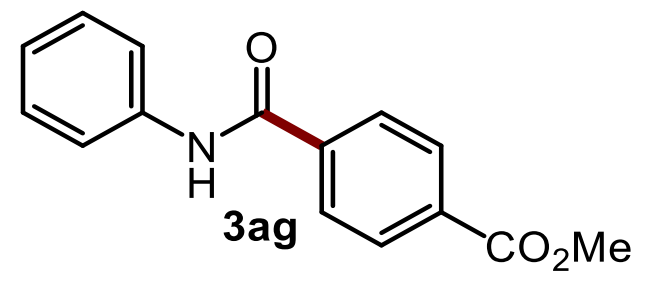

methyl 4-(phenylcarbamoyl)benzoate (3ag): Synthesized according to general procedure $\mathbf{C}$ using masked isocyanate $1 \mathrm{a}(0.128 \mathrm{~g}, 0.600 \mathrm{mmol})$, boroxine $2 \mathrm{~g}(0.146 \mathrm{~g}, 0.300 \mathrm{mmol}), \mathrm{Et}_{3} \mathrm{~N}(0.0836 \mathrm{~mL}, 0.600$ $\mathrm{mmol})$, and $[\mathrm{Rh}(\mathrm{OH})(\mathrm{cod})]_{2}(6.84 \mathrm{mg}, 0.0150 \mathrm{mmol})$. The reaction reached completion within 5 hours. The desired product was purified by column chromatography (25\% EtOAc/ hexanes) yielding an amorphous white solid (0.0933 g, 61\% yield). TLC RF $=0.22$ in $25 \%$ EtOAc/EtOAc. ${ }^{1} \mathrm{H}-\mathrm{NMR}(400 \mathrm{MHz}$, DMSO- $\left.d_{6}\right): \delta 3.89(3 \mathrm{H}, \mathrm{s}), 7.12(1 \mathrm{H}, \mathrm{t}, J=6.5 \mathrm{~Hz}), 7.36(2 \mathrm{H}, \mathrm{t}, J=7.1 \mathrm{~Hz}), 7.79(2 \mathrm{H}, \mathrm{d}, J=7.4 \mathrm{~Hz}), 8.08(4 \mathrm{H}, \mathrm{s})$, $10.44(1 \mathrm{H}, \mathrm{s}) .{ }^{13} \mathrm{C}-\mathrm{NMR}\left(100 \mathrm{MHz}\right.$, DMSO-d $\left.d_{6}\right): \delta 52.9\left(\mathrm{CH}_{3}\right), 120.9(\mathrm{CH}), 124.4(\mathrm{CH}), 128.5(\mathrm{CH}), 129.1(\mathrm{CH})$, 129.6 (CH), 132.5 (C), 139.4 (C), 139.5 (C), 165.2 (C), 166.2 (C). IR (film): 3366, 1714, 1657, $1276 \mathrm{~cm}^{-1}$. HRMS (EI): Exact mass calcd for $\mathrm{C}_{15} \mathrm{H}_{13} \mathrm{NO}_{3}[\mathrm{M}]^{+}:$255.0895. Found: 255.0891.

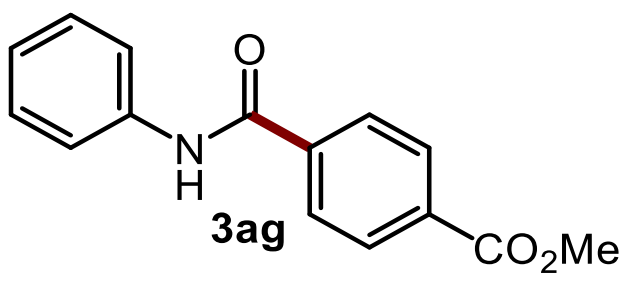

methyl 4-(phenylcarbamoyl)benzoate (3ag): Synthesized according to general procedure $\mathbf{C}$ using masked isocyanate $1 \mathrm{a}(0.128 \mathrm{~g}, 0.600 \mathrm{mmol})$, the organoboron reagent was recrystalized in water before being dehydrated to the boroxine $2 \mathrm{~g}(0.146 \mathrm{~g}, 0.300 \mathrm{mmol}), \mathrm{Et}_{3} \mathrm{~N}(0.0836 \mathrm{~mL}, 0.600 \mathrm{mmol})$, and $[\mathrm{Rh}(\mathrm{OH})(\mathrm{cod})]_{2}\left(1.37 \mathrm{mg}, 3.00 \times 10^{-3} \mathrm{mmol}\right)$. The reaction reached completion within 20 hours. The desired product was extracted from the crude reaction mixture and recrystallized twice ( $50 \%$ Acetone/ 
hexanes) providing a crystalline white solid $\left(0.109 \mathrm{~g}, 71 \%\right.$ yield). ${ }^{1} \mathrm{H}-\mathrm{NMR}\left(400 \mathrm{MHz}, \mathrm{DMSO}-d_{6}\right): \delta 3.89$ $(3 \mathrm{H}, \mathrm{s}), 7.12(1 \mathrm{H}, \mathrm{t}, \mathrm{J}=6.5 \mathrm{~Hz}), 7.36(2 \mathrm{H}, \mathrm{t}, \mathrm{J}=7.1 \mathrm{~Hz}), 7.79(2 \mathrm{H}, \mathrm{d}, J=7.4 \mathrm{~Hz}), 8.08(4 \mathrm{H}, \mathrm{s}), 10.44(1 \mathrm{H}, \mathrm{s}) .{ }^{13} \mathrm{C}-$ NMR $\left(100 \mathrm{MHz}\right.$, DMSO- $\left.d_{6}\right): \delta 52.9\left(\mathrm{CH}_{3}\right), 120.9(\mathrm{CH}), 124.4(\mathrm{CH}), 128.5(\mathrm{CH}), 129.1(\mathrm{CH}), 129.6(\mathrm{CH}), 132.5$ (C), 139.4 (C), 139.5 (C), 165.2 (C), 166.2 (C). IR (film): 3366, 1714, 1657, $1276 \mathrm{~cm}^{-1}$. HRMS (EI): Exact mass calcd for $\mathrm{C}_{15} \mathrm{H}_{13} \mathrm{NO}_{3}[\mathrm{M}]^{+}: 255.0895$. Found: 255.0891 .<smiles>O=C(Nc1ccccc1)c1cccc(F)c1</smiles>

3-fluoro- $\mathbf{N}$-phenylbenzamide (3ah): Synthesized according to general procedure $\mathbf{C}$ using masked isocyanate 1a $(0.128 \mathrm{~g}, 0.600 \mathrm{mmol})$, boroxine $2 \mathrm{~h}(0.110 \mathrm{~g}, 0.300 \mathrm{mmol}), \mathrm{Et}_{3} \mathrm{~N}(0.0836 \mathrm{~mL}, 0.600 \mathrm{mmol})$, and $[\mathrm{Rh}(\mathrm{OH})(\mathrm{cod})]_{2}\left(2.73 \mathrm{mg}, 6.00 \times 10^{-3} \mathrm{mmol}\right)$. The reaction reached completion within 13 hours. The desired product was purified by column chromatography $\left(75 \% \mathrm{CH}_{2} \mathrm{Cl}_{2} /\right.$ hexanes) yielding an amorphous white solid ( $0.101 \mathrm{~g}$, 78\% yield). $\mathrm{TLC} \mathrm{R}_{\mathrm{F}}=0.22$ in $60 \% \mathrm{CH}_{2} \mathrm{Cl}_{2} /$ hexanes. ${ }^{1} \mathrm{H}-\mathrm{NMR}\left(400 \mathrm{MHz}, \mathrm{DMSO}-d_{6}\right): \delta$ $7.11(1 \mathrm{H}, \mathrm{t}, J=7.4 \mathrm{~Hz}), 7.35(2 \mathrm{H}, \mathrm{t}, J=7.5 \mathrm{~Hz}), 7.43(1 \mathrm{H}, \mathrm{dd}, J=2.6,8.3 \mathrm{~Hz}), 7.54-7.60(1 \mathrm{H}, \mathrm{m}), 7.75-7.82(4 \mathrm{H}$, $\mathrm{m}), 10.31(1 \mathrm{H}, \mathrm{s}) .{ }^{13} \mathrm{C}-\mathrm{NMR}\left(100 \mathrm{MHz}\right.$, DMSO-d $\left.d_{6}\right): \delta 115.0(\mathrm{CH}, \mathrm{d}, J=22.86 \mathrm{~Hz}), 118.9(\mathrm{CH}, \mathrm{d}, J=21.13 \mathrm{~Hz})$, $120.9(\mathrm{CH}), 124.3(\mathrm{CH}), 124.4(\mathrm{CH}), 129.1(\mathrm{CH}), 131.0(\mathrm{CH}, \mathrm{d}, \mathrm{J}=8.03 \mathrm{~Hz}), 137.7$ (C, d, J=6.83 Hz), 139.4 (C), $162.4(C, d, J=244.38 \mathrm{~Hz}), 164.6(C, d, J=2.51 \mathrm{~Hz})$. Data is consistent with literature. ${ }^{13}$

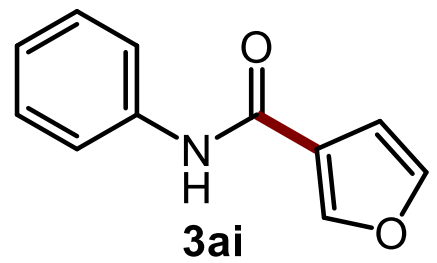

$\mathbf{N}$-phenylfuran-3-carboxamide (3ai): Synthesized according to general procedure $\mathbf{C}$ using masked isocyanate 1a $(0.128 \mathrm{~g}, 0.600 \mathrm{mmol})$, boroxine $2 \mathrm{i}(0.0844 \mathrm{~g}, 0.300 \mathrm{mmol}), \mathrm{Et}_{3} \mathrm{~N}(0.0836 \mathrm{~mL}, 0.600 \mathrm{mmol})$, and $[\mathrm{Rh}(\mathrm{OH})(\mathrm{cod})]_{2}(6.84 \mathrm{mg}, 0.0150 \mathrm{mmol})$. The reaction reached completion within 5 hours. The desired product was purified by column chromatography (98:1:1 $\left.\mathrm{CH}_{2} \mathrm{Cl}_{2} / \mathrm{EtOAc} / \mathrm{AcOH}\right)$ yielding an amorphous white solid (0.0898 g, 80\% yield). TLC $\mathrm{R}_{\mathrm{F}}=27$ in 60\% 98:1:1 $\mathrm{CH}_{2} \mathrm{Cl}_{2} / \mathrm{EtOAc} / \mathrm{AcOH} .{ }^{1} \mathrm{H}-\mathrm{NMR}$ (400 MHz, DMSO- $\left.d_{6}\right): \delta 7.00(1 \mathrm{H}, \mathrm{s}), 7.08(1 \mathrm{H}, \mathrm{t}, J=7.4 \mathrm{~Hz}), 7.34(2 \mathrm{H}, \mathrm{t}, J=7.9 \mathrm{~Hz}), 7.71(2 \mathrm{H}, \mathrm{d}, J=7.8 \mathrm{~Hz})$, $7.79(1 \mathrm{H}, \mathrm{d}, J=1.4 \mathrm{~Hz}), 8.38(1 \mathrm{H}, \mathrm{s}), 9.90(1 \mathrm{H}, \mathrm{s}) .{ }^{13} \mathrm{C}-\mathrm{NMR}\left(100 \mathrm{MHz}, \mathrm{DMSO}-d_{6}\right): \delta 109.7(\mathrm{CH}), 120.7(\mathrm{CH})$, 123.5 (C), $124.1(\mathrm{CH}), 129.1(\mathrm{CH}), 139.3$ (C), $144.7(\mathrm{CH}), 146.3(\mathrm{CH}), 160.9$ (C). IR (film): 3329, $1655 \mathrm{~cm}^{-1}$. HRMS (EI): Exact mass calcd for $\mathrm{C}_{11} \mathrm{H}_{9} \mathrm{NO}_{2}[\mathrm{M}]^{+}:$187.0633. Found: 187.0611 . 


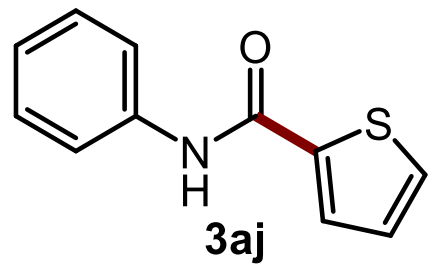

$\mathbf{N}$-phenylthiophene-2-carboxamide (3aj): Synthesized according to general procedure $\mathbf{C}$ using masked isocyanate $1 \mathrm{a}(0.128 \mathrm{~g}, 0.600 \mathrm{mmol})$, boroxine $2 \mathrm{j}(0.0989 \mathrm{~g}, 0.300 \mathrm{mmol}), \mathrm{Et}_{3} \mathrm{~N}(0.0836 \mathrm{~mL}, 0.600 \mathrm{mmol})$, and $[\mathrm{Rh}(\mathrm{OH})(\mathrm{cod})]_{2}(6.84 \mathrm{mg}, 0.0150 \mathrm{mmol})$. The reaction reached completion within 16 hours. The desired product was purified by column chromatography $\left(\mathrm{CH}_{2} \mathrm{Cl}_{2}\right.$ to $\left.99 \% \mathrm{CH}_{2} \mathrm{Cl}_{2} / \mathrm{EtOAc}\right)$ yielding an amorphous white solid (0.0920 g, 75\% yield). TLC $\mathrm{R}_{\mathrm{F}}=0.18$ in $\mathrm{CH}_{2} \mathrm{Cl}_{2} .{ }^{1} \mathrm{H}-\mathrm{NMR}\left(400 \mathrm{MHz}, \mathrm{DMSO}-d_{6}\right): \delta$ $7.08(1 \mathrm{H}, \mathrm{t}, J=7.2 \mathrm{~Hz}), 7.34(2 \mathrm{H}, \mathrm{t}, J=7.7 \mathrm{~Hz}), 7.64(2 \mathrm{H}, \mathrm{s}), 7.76(2 \mathrm{H}, \mathrm{d}, J=7.9 \mathrm{~Hz}), 8.35(1 \mathrm{H}, \mathrm{s}), 10.05(1 \mathrm{H}, \mathrm{s})$. ${ }^{13} \mathrm{C}-N M R\left(100 \mathrm{MHz}\right.$, DMSO- $\left.d_{6}\right): \delta 120.8(\mathrm{CH}), 124.0(\mathrm{CH}), 127.3(\mathrm{CH}), 127.7(\mathrm{CH}), 129.1(\mathrm{CH}), 130.1(\mathrm{CH})$, 138.3 (C), 139.5 (C), 161.4 (C). Data is consistent with literature. ${ }^{21}$<smiles>O=C(Nc1ccccc1)c1ccc(F)nc1</smiles>

6-fluoro- $\boldsymbol{N}$-phenylnicotinamide (3ak): Synthesized according to general procedure $\mathbf{D}$ using masked isocyanate $1 \mathrm{a}(0.128 \mathrm{~g}, 0.600 \mathrm{mmol})$, boroxine $2 \mathrm{k}(0.221 \mathrm{~g}, 0.600 \mathrm{mmol}), \mathrm{Et}_{3} \mathrm{~N}(0.0836 \mathrm{~mL}, 0.600 \mathrm{mmol})$, and $[\mathrm{Rh}(\mathrm{OH})(\mathrm{cod})]_{2}(6.84 \mathrm{mg}, 0.0150 \mathrm{mmol})$. The reaction reached completion within 10 hours. The desired product was purified by column chromatography (95\% $\mathrm{CH}_{2} \mathrm{Cl}_{2} / \mathrm{EtOAc}$ to $\left.90 \% \mathrm{CH}_{2} \mathrm{Cl}_{2} / \mathrm{EtOAc}\right)$ yielding an amorphous white solid $\left(0.103 \mathrm{~g}\right.$, 79\% yield). TLC $\mathrm{R}_{\mathrm{F}}=0.16$ in $95 \% \mathrm{CH}_{2} \mathrm{Cl}_{2} / \mathrm{EtOAc}$. ${ }^{1} \mathrm{H}-\mathrm{NMR}(400$ MHz, DMSO- $\left.d_{6}\right): \delta 7.13(3 \mathrm{H}, \mathrm{t}, J=7.4 \mathrm{~Hz}), 7.33-7.38(3 \mathrm{H}, \mathrm{m}), 7.76(2 \mathrm{H}, \mathrm{d}, J=7.7 \mathrm{~Hz}), 8.50(1 \mathrm{H}, \mathrm{dt}, J=2.5 \mathrm{~Hz}$, $8.2 \mathrm{~Hz}), 8.82(1 \mathrm{H}, \mathrm{d}, J=2.3 \mathrm{~Hz}), 10.44(1 \mathrm{H}, \mathrm{s}) .{ }^{13} \mathrm{C}-\mathrm{NMR}\left(100 \mathrm{MHz}, \mathrm{DMSO}-d_{6}\right): \delta 109.9(\mathrm{CH}, \mathrm{d}, J=37.7 \mathrm{~Hz})$, $120.9(\mathrm{CH}), 124.5(\mathrm{CH}), 129.2(\mathrm{CH}), 129.8(\mathrm{C}, \mathrm{d}, \mathrm{J}=4.3 \mathrm{~Hz}), 139.2(\mathrm{C}), 142.4(\mathrm{CH}, \mathrm{d}, J=9.1 \mathrm{~Hz}), 148.2(\mathrm{CH}, \mathrm{d}$, $J=16.3 \mathrm{~Hz}$ ), 163.3 (C), 164.8 (C, d, J=240.2 Hz). IR (film): 3346, 1651, $1254 \mathrm{~cm}^{-1}$. HRMS (EI): Exact mass calcd for $\mathrm{C}_{12} \mathrm{H}_{9} \mathrm{~N}_{2} \mathrm{OF}[\mathrm{M}]^{+}: 216.0699$. Found: 216.0700 . 


\section{7-Gram-Scale Reaction}

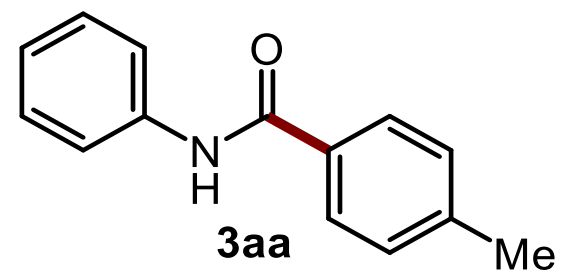

4-methyl- $\boldsymbol{N}$-phenylbenzamide (3aa): An oven-dried $50 \mathrm{~mL}$ round-bottom flask equipped with a stir bar was cooled under a stream of argon followed by the addition of $[\mathrm{Rh}(\mathrm{OH})(\mathrm{cod})]_{2}(21.4 \mathrm{mg}, 0.0469 \mathrm{mmol})$, boroxine 2a $(0.829 \mathrm{~g}, 7.03 \mathrm{mmol})$, and THF $(9.40 \mathrm{~mL})$. Argon was bubbled through the solution for 2 minutes upon which compound $1 \mathrm{a}(1.00 \mathrm{~g}, 4.69 \mathrm{mmol})$ and $\mathrm{Et}_{3} \mathrm{~N}(0.653 \mathrm{~mL}, 4.69 \mathrm{mmol})$ were added. The flask was sealed with a septum, purged with argon, and placed in a $50{ }^{\circ} \mathrm{C}$ oil bath. The reaction was monitored for the disappearance of starting material. The reaction was deemed complete in 24 hours. The reaction was concentrated under reduced pressure, diluted with EtOAc $(200 \mathrm{~mL})$ and extracted $1 \mathrm{M}$ $\mathrm{NaOH}(1 \times 100 \mathrm{~mL})$, saturated $\mathrm{NaHCO}_{3}(1 \times 100 \mathrm{~mL})$, and brine $(1 \times 100 \mathrm{~mL})$. The organic layer was collected, dried over $\mathrm{Na}_{2} \mathrm{SO}_{4}$, and concentrated under reduced pressure. The desired product was purified by column chromatography $\left(99 \% \mathrm{CH}_{2} \mathrm{Cl}_{2} / \mathrm{Et}_{3} \mathrm{~N}\right)$ yielding an amorphous white solid $(0.788 \mathrm{~g}, 66 \%$ yield). TLC $\mathrm{R}_{\mathrm{F}}=0.42$ in $99 \% \mathrm{CH}_{2} \mathrm{Cl}_{2} / \mathrm{Et}_{3} \mathrm{~N}$. ${ }^{1} \mathrm{H}-\mathrm{NMR}\left(400 \mathrm{MHz}\right.$, DMSO-d $\left.d_{6}\right): \delta 2.34(3 \mathrm{H}, \mathrm{s}), 7.06(1 \mathrm{H}, \mathrm{t}, J=7.4$ $\mathrm{Hz}), 7.27-7.34(3 \mathrm{H}, \mathrm{m}), 7.79(1 \mathrm{H}, \mathrm{q}, \mathrm{J}=7.5 \mathrm{~Hz}), 7.87(1 \mathrm{H}, \mathrm{d}, \mathrm{J}=8.2 \mathrm{~Hz}), 10.15(1 \mathrm{H}, \mathrm{s}) .{ }^{13} \mathrm{C}-\mathrm{NMR}(100 \mathrm{MHz}$, DMSO- $\left.d_{6}\right): \delta 21.4\left(\mathrm{CH}_{3}\right), 120.8(\mathrm{CH}), 123.9(\mathrm{CH}), 128.1(\mathrm{CH}), 129.0(\mathrm{CH}), 129.3(\mathrm{CH}), 132.6(\mathrm{C}), 139.7(\mathrm{C})$, 142.0 (C), 165.8 (C). Data is consistent with literature.

\section{8-Synthesis of Amides from Table 2c}

General procedure D (high [Rh], high [RBO $]_{3}$, low [THF]): An oven-dried microwave vial equipped with a stir bar was cooled under a stream of argon followed by the addition of $[\mathrm{Rh}(\mathrm{OH})(\mathrm{cod})]_{2}(0.0150 \mathrm{mmol}$, 0.0250 equiv.), organoboron reagent $(0.600 \mathrm{mmol}, 1.00$ equiv.), and THF (6.00 mL). Argon was bubbled through the solution for 2 minutes upon which the blocked isocyanate $(0.600 \mathrm{mmol}, 1.00$ equiv.) and $\mathrm{Et}_{3} \mathrm{~N}(0.600 \mathrm{mmol}, 1.00$ equiv.) were added. The microwave vial was sealed, purged with argon, and placed in a $50{ }^{\circ} \mathrm{C}$ oil bath. The reaction was monitored for the disappearance of starting material. Upon completion, the reaction was concentrated under reduced pressure, diluted with EtOAc $(40 \mathrm{~mL})$ and extracted $1 \mathrm{M} \mathrm{NaOH}(1 \times 30 \mathrm{~mL})$, saturated $\mathrm{NaHCO}_{3}(1 \times 30 \mathrm{~mL})$, and brine $(1 \times 30 \mathrm{~mL})$. The organic layer was collected, dried over $\mathrm{Na}_{2} \mathrm{SO}_{4}$, and concentrated under reduced pressure. The crude mixture was purified by silica gel column chromatography providing the desired product. 


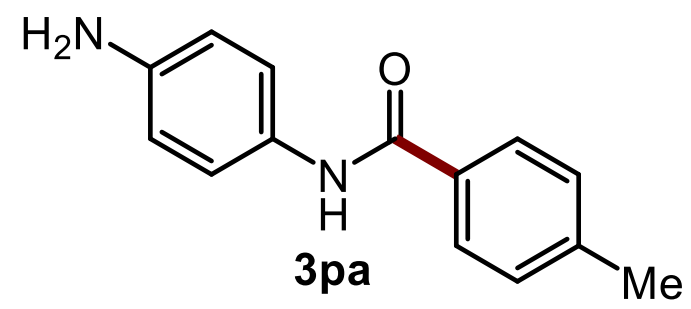

$\mathbf{N}$-(4-aminophenyl)-4-methylbenzamide (3pa): Synthesized according to general procedure $\mathbf{D}$ using masked isocyanate $1 \mathrm{p}(0.137 \mathrm{~g}, 0.600 \mathrm{mmol})$, organoboron reagent $2 \mathrm{a}(0.244 \mathrm{~g}, 1.95 \mathrm{mmol}, 3.3$ equiv "Ar-B"), $E t_{3} \mathrm{~N}(0.0836 \mathrm{~mL}, 0.600 \mathrm{mmol})$, and $[\mathrm{Rh}(\mathrm{OH})(\mathrm{cod})]_{2}(6.84 \mathrm{mg}, 0.0150 \mathrm{mmol})$. The reaction reached completion within 12 hours. The desired product was purified by column chromatography (75\% $\mathrm{CH}_{2} \mathrm{Cl}_{2}$ /EtOAC) yielding an amorphous white solid ( $0.114 \mathrm{~g}$, 84\% yield). TLC $\mathrm{R}_{\mathrm{F}}=0.36$ in $75 \%$ $\mathrm{CH}_{2} \mathrm{Cl}_{2}$ /EtOAc. ${ }^{1} \mathrm{H}-\mathrm{NMR}\left(400 \mathrm{MHz}\right.$, DMSO- $\left.d_{6}\right): \delta 2.33(3 \mathrm{H}, \mathrm{s}), 4.88(2 \mathrm{H}, \mathrm{s}), 6.51(1 \mathrm{H}, \mathrm{d}, J=8.7 \mathrm{~Hz}), 7.26(1 \mathrm{H}$, d, $J=8.0 \mathrm{~Hz}), 7.34(1 \mathrm{H}, \mathrm{d}, J=8.7 \mathrm{~Hz}), 7.80(1 \mathrm{H}, \mathrm{d}, J=8.1 \mathrm{~Hz}), 9.75(1 \mathrm{H}, \mathrm{s}) \cdot{ }^{13} \mathrm{C}-\mathrm{NMR}\left(100 \mathrm{MHz}, \mathrm{DMSO}-d_{6}\right): \delta$ $169.7(\mathrm{C}), 150.3\left(\mathrm{CH}_{2}\right), 146.2\left(\mathrm{CH}_{2}\right), 137.7\left(\mathrm{CH}_{2}\right), 134.0(\mathrm{CH}), 133.4\left(\mathrm{CH}_{2}\right), 132.7(\mathrm{CH}), 127.5(\mathrm{CH}), 118,9$ $(\mathrm{CH}), 26.2\left(\mathrm{CH}_{3}\right)$. Data is consistent with literature. ${ }^{22}$<smiles>Cc1ccc(C(=O)Nc2cccc(N)c2)cc1</smiles>

$\mathbf{N}$-(3-aminophenyl)-4-methylbenzamide (3qa): Synthesized according to general procedure $\mathbf{D}$ using masked isocyanate $1 \mathrm{q}(0.137 \mathrm{~g}, 0.600 \mathrm{mmol})$, organoboron reagent $\mathbf{2 a}(0.244 \mathrm{~g}, 1.95 \mathrm{mmol}, 3.3$ equiv "Ar-B"), $\mathrm{Et}_{3} \mathrm{~N}(0.0836 \mathrm{~mL}, 0.600 \mathrm{mmol})$, and $[\mathrm{Rh}(\mathrm{OH})(\mathrm{cod})]_{2}(6.84 \mathrm{mg}, 0.0150 \mathrm{mmol})$. The reaction reached completion within 12 hours. The desired product was purified by column chromatography (75\% $\mathrm{CH}_{2} \mathrm{Cl}_{2}$ /EtOAc) yielding an amorphous white solid ( $0.113 \mathrm{~g}$, 83\% yield). TLC $\mathrm{R}_{\mathrm{F}}=0.29$ in $75 \%$ $\mathrm{CH}_{2} \mathrm{Cl}_{2}$ /EtOAc. ${ }^{1} \mathrm{H}-\mathrm{NMR}\left(400 \mathrm{MHz}\right.$, Acetone- $\left.d_{6}\right): \delta 2.35(3 \mathrm{H}, \mathrm{s}), 4.63(2 \mathrm{H}, \mathrm{s}, \mathrm{br}), 6.42-6.45(1 \mathrm{H}, \mathrm{m}), 6.98-$ $7.04(2 \mathrm{H}, \mathrm{d}), 7.25(2 \mathrm{H}, \mathrm{d}, J=8.0 \mathrm{~Hz}), 7.30(1 \mathrm{H}, \mathrm{s}), 7.86(2 \mathrm{H}, \mathrm{d}, J=8.2 \mathrm{~Hz}), 9.29(1 \mathrm{H}, \mathrm{s}) .{ }^{13} \mathrm{C}-\mathrm{NMR}(100 \mathrm{MHz}$, Acetone- $\left.d_{6}\right)$ : $\delta 20.6\left(\mathrm{CH}_{3}\right), 106.4(\mathrm{CH}), 109.0(\mathrm{CH}), 110.2(\mathrm{CH}), 127.5(\mathrm{CH}), 129.0(\mathrm{CH}), 129.1(\mathrm{CH}), 132.9$ (C), 140.2 (C), 141.7 (C), 148.9 (C), 165.4 (C). Data is consistent with literature. ${ }^{23}$

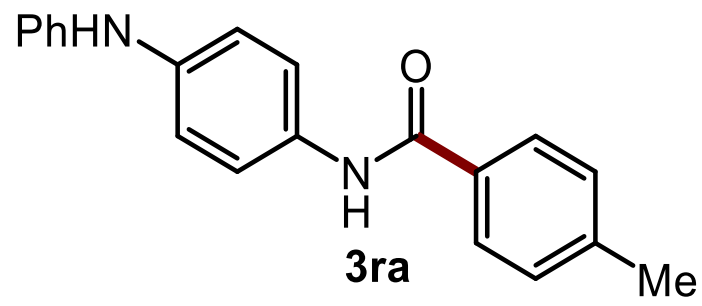

4-methyl- $\boldsymbol{N}$-(4-(phenylamino)phenyl)benzamide (3ra): Synthesized according to general procedure D using masked isocyanate $1 \mathrm{r}(0.183 \mathrm{~g}, 0.600 \mathrm{mmol})$, organoboron reagent $2 \mathrm{a}(0.244 \mathrm{~g}, 1.95 \mathrm{mmol}, 3.3$ equiv “Ar-B"), $\mathrm{Et}_{3} \mathrm{~N}(0.0836 \mathrm{~mL}, 0.600 \mathrm{mmol})$, and $[\mathrm{Rh}(\mathrm{OH})(\mathrm{cod})]_{2}(6.84 \mathrm{mg}, 0.0150 \mathrm{mmol})$. The reaction reached completion within 16 hours. The desired product was purified by column chromatography (30\% 
EtOAC/ hexanes) yielding an amorphous white solid ( $0.153 \mathrm{~g}$, 85\% yield). TLC $R_{F}=0.47$ in $40 \%$ EtOAc/hexanes. ${ }^{1} \mathrm{H}-\mathrm{NMR}\left(400 \mathrm{MHz}, \mathrm{DMSO}-d_{6}\right): \delta 2.37(3 \mathrm{H}, \mathrm{s}), 6.77(1 \mathrm{H}, \mathrm{t}, J=7.3 \mathrm{~Hz}), 7.02-7.09(4 \mathrm{H}, \mathrm{m})$, $7.20(2 \mathrm{H}, \mathrm{t}, \mathrm{J}=7.6 \mathrm{~Hz}), 7.31(2 \mathrm{H}, \mathrm{d}, \mathrm{J}=8.0 \mathrm{~Hz}), 7.65(2 \mathrm{H}, \mathrm{d}, \mathrm{J}=8.9 \mathrm{~Hz}), 7.87(2 \mathrm{H}, \mathrm{d}, \mathrm{J}=8.2 \mathrm{~Hz}), 8.08(1 \mathrm{H}, \mathrm{s})$, $10.03(1 \mathrm{H}, \mathrm{s}) .{ }^{13} \mathrm{C}-\mathrm{NMR}\left(100 \mathrm{MHz}, \mathrm{DMSO}-d_{6}\right): \delta 21.5(\mathrm{CH}), 116.4(\mathrm{CH}), 118.0(\mathrm{CH}), 119.5(\mathrm{CH}), 122.2(\mathrm{CH})$, $128.0(\mathrm{CH}), 129.3(\mathrm{CH}), 129.6(\mathrm{CH}), 132.4$ (C), 132.7 (C), 139.6 (C), 141.7 (C), 144.5 (C), 165.3 (C). IR (film): $3403,3324,1641,1304 \mathrm{~cm}^{-1}$. HRMS (EI): Exact mass calcd for $\mathrm{C}_{20} \mathrm{H}_{18} \mathrm{~N}_{2} \mathrm{O}[\mathrm{M}]^{+}$: 302.1419. Found: 302.1426 .

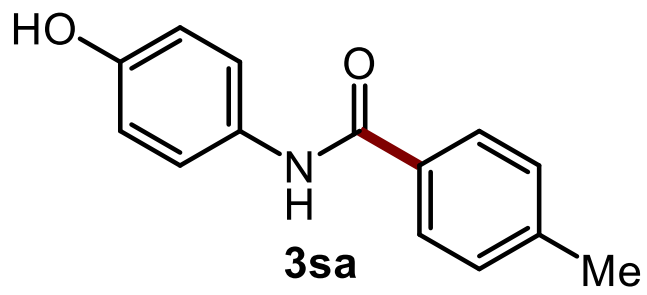

$\mathbf{N}$-(4-hydroxyphenyl)-4-methylbenzamide (3sa): Synthesized according to general procedure $\mathbf{D}$ using masked isocyanate $1 \mathrm{~s}(0.138 \mathrm{~g}, 0.600 \mathrm{mmol})$, organoboron reagent $2 \mathrm{a}(0.244 \mathrm{~g}, 1.95 \mathrm{mmol}, 3.3$ equiv "Ar-B"), $E t_{3} \mathrm{~N}(0.0836 \mathrm{~mL}, 0.600 \mathrm{mmol})$, and $[\mathrm{Rh}(\mathrm{OH})(\mathrm{cod})]_{2}(6.84 \mathrm{mg}, 0.0150 \mathrm{mmol})$. The reaction reached completion within 18 hours. The reaction was concentrated under reduced pressure, resuspended in EtOAc $(40 \mathrm{~mL})$ and extracted with $1 \mathrm{M} \mathrm{NaOH}(1 \times 40 \mathrm{~mL})$. The aqueous phase was quenched with a saturated $\mathrm{NaHCO}_{3}(40 \mathrm{~mL})$ and subsequently extracted with EtOAc $(3 \times 40 \mathrm{~mL})$. The organic phases were combined, dried over $\mathrm{Na}_{2} \mathrm{SO}_{4}$, and concentrated under reduced pressure. The crude extract was purified by column chromatography $\left(20 \% \mathrm{EtOAc} / \mathrm{CH}_{2} \mathrm{Cl}_{2}\right)$ yielding an amorphous white solid (0.121 g, 89\% yield). TLC $\mathrm{R}_{\mathrm{F}}=0.11$ in $10 \% \mathrm{EtOAc} / \mathrm{CH}_{2} \mathrm{Cl}_{2} .{ }^{1} \mathrm{H}-\mathrm{NMR}\left(400 \mathrm{MHz}\right.$, DMSO- $\left.d_{6}\right): \delta 2.37(3 \mathrm{H}$, s), 6.72-6.74 (2H, m), $7.31(2 \mathrm{H}, \mathrm{d}, J=8.0 \mathrm{~Hz}), 7.52(2 \mathrm{H}, \mathrm{d}, J=8.9 \mathrm{~Hz}), 7.85(2 \mathrm{H}, \mathrm{d}, J=8.13 \mathrm{~Hz}), 9.23(1 \mathrm{H}, \mathrm{s})$, $9.93(1 \mathrm{H}, \mathrm{s}) .{ }^{13} \mathrm{C}-\mathrm{NMR}\left(100 \mathrm{MHz}\right.$, DMSO- $\left.d_{6}\right): \delta 21.5(\mathrm{CH}), 115.4(\mathrm{CH}), 122.7(\mathrm{CH}), 128.0(\mathrm{CH}), 129.3(\mathrm{CH})$, 131.22 (C), 132.7 (C), 141.7 (C), 154.1 (C), 165.2 (C). IR (film): 3387, 3326, 1648, $1225 \mathrm{~cm}^{-1}$. HRMS (EI): Exact mass calcd for $\mathrm{C}_{14} \mathrm{H}_{13} \mathrm{NO}_{2}[\mathrm{M}]^{+}: 227.0946$. Found: 227.0943.

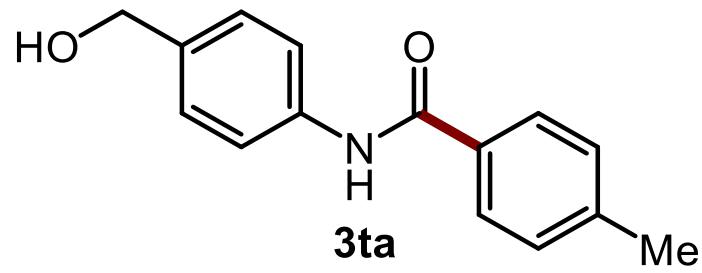

$\mathbf{N}$-(4-(hydroxymethyl)phenyl)-4-methylbenzamide (3ta): Synthesized according to general procedure D using masked isocyanate $1 \mathrm{t}(0.146 \mathrm{~g}, 0.600 \mathrm{mmol})$, organoboron reagent $2 \mathrm{a}(0.244 \mathrm{~g}, 1.95 \mathrm{mmol}, 3.3$ equiv “Ar-B"), $E t_{3} \mathrm{~N}(0.0836 \mathrm{~mL}, 0.600 \mathrm{mmol})$, and $[\mathrm{Rh}(\mathrm{OH})(\mathrm{cod})]_{2}(6.84 \mathrm{mg}, 0.0150 \mathrm{mmol})$. The reaction reached completion within 10 hours. The crude extract was dry loaded onto silica gel $(\mathrm{MeOH})$ and purified by column chromatography $\left(40 \% \mathrm{EtOAc} / \mathrm{CH}_{2} \mathrm{Cl}_{2}\right.$ to $50 \% \mathrm{EtOAc} / \mathrm{CH}_{2} \mathrm{Cl}_{2}$ ) yielding an amorphous white solid $\left(0.134 \mathrm{~g}\right.$, 93\% yield). TLC RF $=0.45$ in $50 \% \mathrm{EtOAC} / \mathrm{CH}_{2} \mathrm{Cl}_{2} .{ }^{1} \mathrm{H}-\mathrm{NMR}$ (400 MHz, DMSO- $\left.d_{6}\right): \delta 2.38$ $(3 \mathrm{H}, \mathrm{s}), 4.49(2 \mathrm{H}, \mathrm{d}, J=5.6 \mathrm{~Hz}), 5.15(1 \mathrm{H}, \mathrm{t}, J=5.7 \mathrm{~Hz}), 7.31(4 \mathrm{H}, \mathrm{t}, J=8.8 \mathrm{~Hz}), 7.75(2 \mathrm{H}, \mathrm{d}, J=8.5 \mathrm{~Hz}), 7.89(2 \mathrm{H}$, d, J=8.1 Hz), $10.14(1 \mathrm{H}, \mathrm{s}) .{ }^{13} \mathrm{C}-\mathrm{NMR}\left(100 \mathrm{MHz}, \mathrm{DMSO}-\mathrm{d}_{6}\right): \delta 21.5(\mathrm{CH}), 63.2\left(\mathrm{CH}_{2}\right), 120.6(\mathrm{CH}), 127.3(\mathrm{CH})$, 
128.1 (CH), 129.3 (CH), 132.6 (C), 138.2 (C), 138.3 (C), 141.9 (C), 165.7 (C). IR (film): 3325, 1649, $1002 \mathrm{~cm}^{-}$

1. HRMS (EI): Exact mass calcd for $\mathrm{C}_{15} \mathrm{H}_{15} \mathrm{NO}_{2}[\mathrm{M}]^{+}:$: 241.1103. Found: 241.1111 .

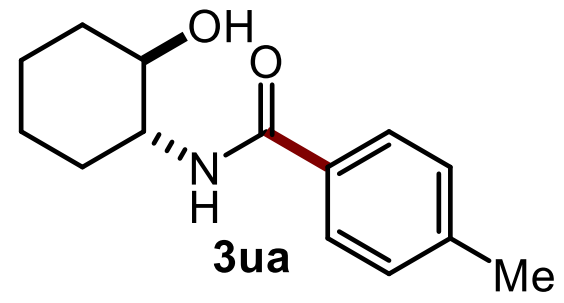

$\mathbf{N}$-((1R,2R)-2-hydroxycyclohexyl)-4-methylbenzamide (3ua): Synthesized according to general procedure $\mathbf{D}$ using masked isocyanate $1 \mathbf{u}(0.141 \mathrm{~g}, 0.600 \mathrm{mmol})$, organoboron reagent $2 \mathrm{a}(0.244 \mathrm{~g}, 1.95$ mmol, 3.3 equiv "Ar- $\left.\mathrm{B}^{\prime}\right)$, $\mathrm{Et} \mathrm{H}_{3} \mathrm{~N}(0.0836 \mathrm{~mL}, 0.600 \mathrm{mmol})$, and $[\mathrm{Rh}(\mathrm{OH})(\mathrm{cod})]_{2}(6.84 \mathrm{mg}, 0.0150 \mathrm{mmol})$. The reaction reached completion within 18 hours. The desired product was purified by column chromatography $\left(25 \% \mathrm{EtOAC} / \mathrm{CH}_{2} \mathrm{Cl}_{2}\right)$ yielding an amorphous white solid $(0.115 \mathrm{~g}, 82 \%$ yield $) . \mathrm{TLC} \mathrm{R}_{\mathrm{F}}=$ 0.16 in $25 \%$ EtOAc/ $\mathrm{CH}_{2} \mathrm{Cl}_{2} .{ }^{1} \mathrm{H}-\mathrm{NMR}\left(400 \mathrm{MHz}\right.$, DMSO- $\left.d_{6}\right): \delta 1.20-1.28(4 \mathrm{H}, \mathrm{m}), 1.61-1.66(2 \mathrm{H}, \mathrm{m}), 1.82-$ $1.91(2 \mathrm{H}, \mathrm{m}), 2.35(3 \mathrm{H}, \mathrm{s}), 3.37-3.43(1 \mathrm{H}, \mathrm{m}), 3.56-3.64(1 \mathrm{H}, \mathrm{m}), 4.58(1 \mathrm{H}, \mathrm{d}, J=5.2 \mathrm{~Hz}), 7.25(2 \mathrm{H}, \mathrm{d}, \mathrm{J}=8.0$ $\mathrm{Hz}), 7.76(2 \mathrm{H}, \mathrm{d}, J=8.1 \mathrm{~Hz}), 7.99(1 \mathrm{H}, \mathrm{d}, J=7.9 \mathrm{~Hz}) .{ }^{13} \mathrm{C}-\mathrm{NMR}\left(300 \mathrm{MHz}, \mathrm{DMSO}-d_{6}\right): \delta 21.4\left(\mathrm{CH}_{3}\right), 24.6\left(\mathrm{CH}_{2}\right)$, $24.9\left(\mathrm{CH}_{2}\right), 31.8\left(\mathrm{CH}_{2}\right), 35.0\left(\mathrm{CH}_{2}\right), 55.7(\mathrm{CH}), 71.6(\mathrm{CH}), 127.8(\mathrm{CH}), 129.0(\mathrm{CH}), 132.7(\mathrm{C}), 141.1(\mathrm{C}), 166.4$ (C). IR (film): 3470, 3272, 1748, 1614, 1331, $1046 \mathrm{~cm}^{-1}$. HRMS (EI): Exact mass calcd for $\mathrm{C}_{14} \mathrm{H}_{19} \mathrm{NO}_{2}[\mathrm{M}]^{+}$: 233.1416. Found: $233.1424 .[\alpha]^{20} \mathrm{D}+39.4^{\circ}(c 0.80, \mathrm{MeOH})$.

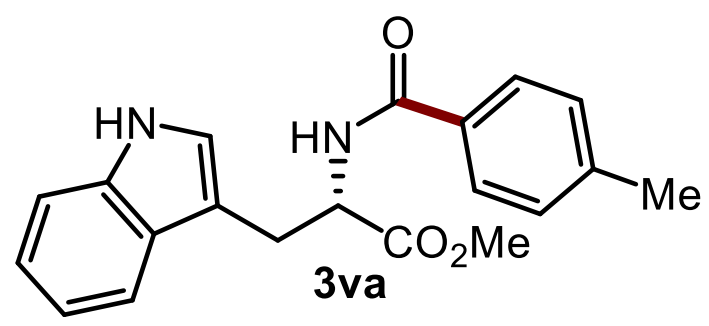

(S)-methyl 3-(1H-indol-3-yl)-2-(4-methylbenzamido)propanoate (3va): Synthesized according to general procedure $\mathbf{D}$ using masked isocyanate $1 \mathbf{v}(0.203 \mathrm{~g}, 0.600 \mathrm{mmol})$, organoboron reagent $2 \mathrm{a}(0.244 \mathrm{~g} 1.95$ mmol, 3.3 equiv "Ar-B"), $E t_{3} \mathrm{~N}(0.0836 \mathrm{~mL}, 0.600 \mathrm{mmol})$, and $[\mathrm{Rh}(\mathrm{OH})(\mathrm{cod})]_{2}(6.84 \mathrm{mg}, 0.0150 \mathrm{mmol})$ at $80^{\circ} \mathrm{C}$. The reaction reached completion within 20 hours. The desired product was purified by column chromatography ( $5 \% \mathrm{EtOAC} / \mathrm{CH}_{2} \mathrm{Cl}_{2}$ ) yielding a white foam $\left(0.145 \mathrm{~g}, 72 \%\right.$ yield). TLC $\mathrm{R}_{\mathrm{F}}=0.24$ in $5 \%$ EtOAc/ $\mathrm{CH}_{2} \mathrm{Cl}_{2} .{ }^{1} \mathrm{H}-\mathrm{NMR}\left(400 \mathrm{MHz}, \mathrm{CDCl}_{3}\right): \delta 2.34(3 \mathrm{H}, \mathrm{s}), 3.40-3.45(2 \mathrm{H}, \mathrm{m}), 3.67(3 \mathrm{H}, \mathrm{s}), 5.13(1 \mathrm{H}, \mathrm{dt}, J=$ 5.3, 7.7 Hz), $6.75(1 \mathrm{H}, \mathrm{d}, J=7.7 \mathrm{~Hz}), 6.93(1 \mathrm{H}, \mathrm{d}, J=2.4 \mathrm{~Hz}), 7.07(1 \mathrm{H}, \mathrm{t}, J=7.0 \mathrm{~Hz}), 7.11-7.17(3 \mathrm{H}, \mathrm{m}), 7.30$ $(1 \mathrm{H}, \mathrm{d}, J=8.1 \mathrm{~Hz}), 7.53-7.58(3 \mathrm{H}, \mathrm{m}), 8.71(1 \mathrm{H}, \mathrm{s}) .{ }^{13} \mathrm{C}-\mathrm{NMR}\left(100 \mathrm{MHz}, \mathrm{CDCl}_{3}\right): \delta 21.5\left(\mathrm{CH}_{3}\right), 27.7\left(\mathrm{CH}_{2}\right), 52.4$ $(\mathrm{CH}), 53.5\left(\mathrm{CH}_{3}\right), 109.9(\mathrm{C}), 111.4(\mathrm{CH}), 118.6(\mathrm{CH}), 119.7(\mathrm{CH}), 122.2(\mathrm{CH}), 123.0(\mathrm{CH}), 127.1(\mathrm{CH}), 127.7$ (C), 129.2 (CH), 131.0 (C), 136.2 (C), 142.2 (C), 167.0 (C), 172.5 (C). IR (film): 3393, 3294, 1732, 1639, $1209 \mathrm{~cm}^{-1}$. HRMS (ESI): Exact mass calcd for $\mathrm{C}_{20} \mathrm{H}_{20} \mathrm{~N}_{2} \mathrm{O}_{3} \mathrm{Na}[\mathrm{M}+\mathrm{Na}]^{+}: 359.1372$. Found: 359.1375 . [ $\left.\alpha\right]^{20}{ }_{D}-$ $26.9^{\circ}$ (c 1.30, MeCN). Chiral HPLC: ChiralPak column AD-H i-PrOH/Hexane $=15: 85,1.0 \mathrm{~mL} / \mathrm{min}, 230$ $\mathrm{nm}, t=17.7 \mathrm{~min}, 43.1 \mathrm{~min}$. 


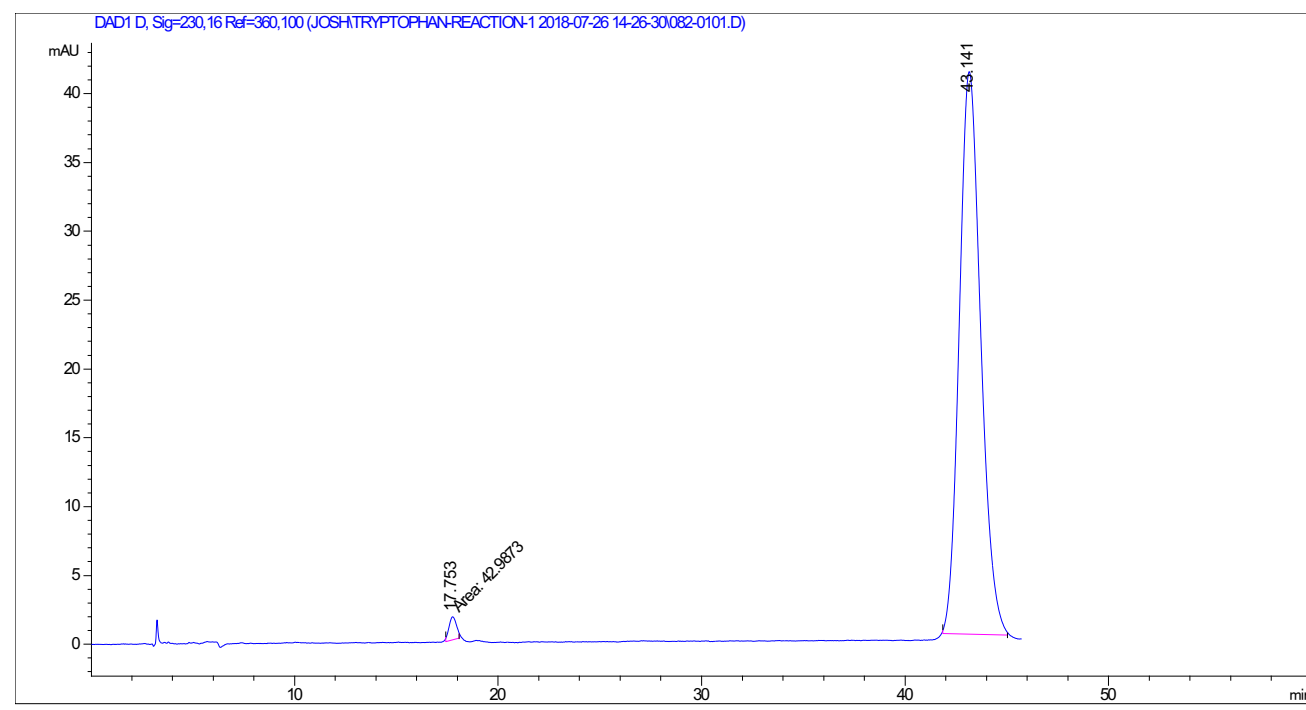

\# Time Area Height Width Area\% Symmetry

$\begin{array}{lllllll}1 & 17.753 & 43 & 1.7 & 0.4224 & 1.415 & 0.938\end{array}$

$\begin{array}{lllllll}2 & 43.141 & 2994.6 & 40.9 & 1.0928 & 98.585 & 0.813\end{array}$

Figure S5. HPLC trace and data of 3va showing $97 \%$ ee

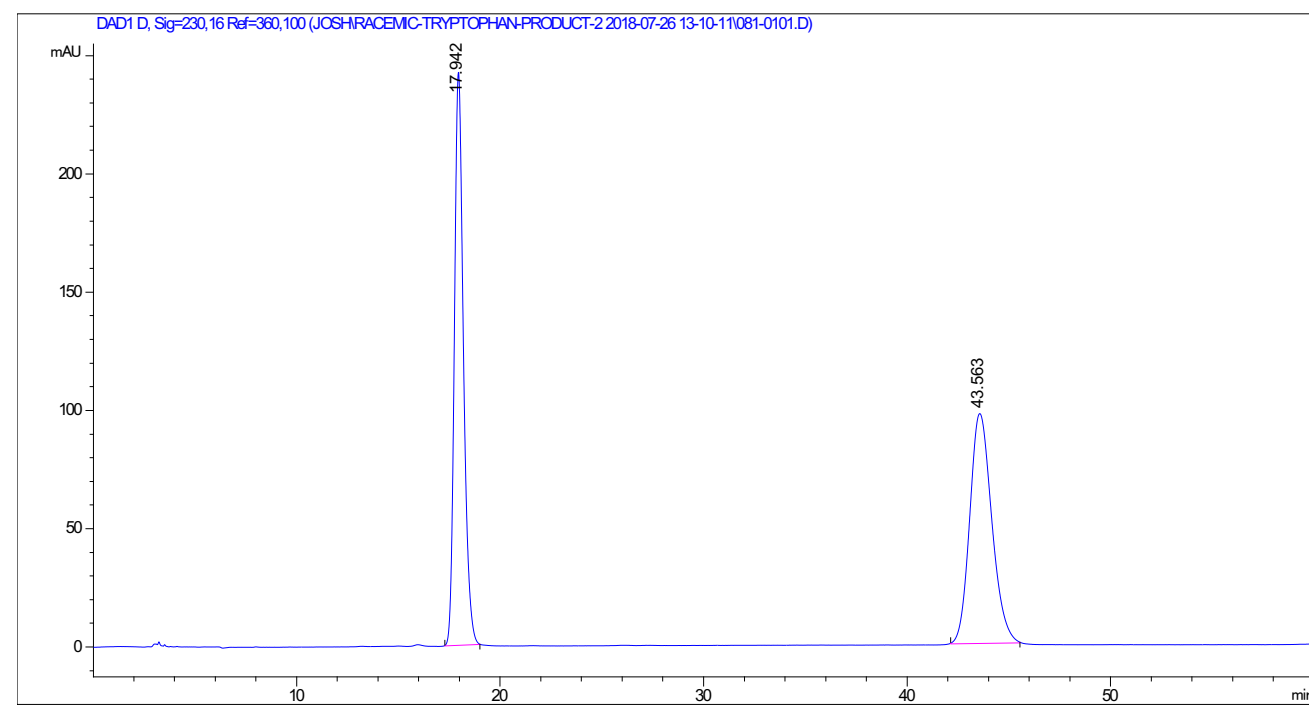

\# $\quad$ Time Area Height Width Area\% Symmetry

$\begin{array}{lllllll}1 & 17.942 & 7515.7 & 242.3 & 0.4718 & 50.616 & 0.764\end{array}$

$\begin{array}{lllllll}2 & 43.563 & 7332.9 & 97.2 & 1.1477 & 49.384 & 0.795\end{array}$

Figure S6. HPLC trace and data of racemic 3va 


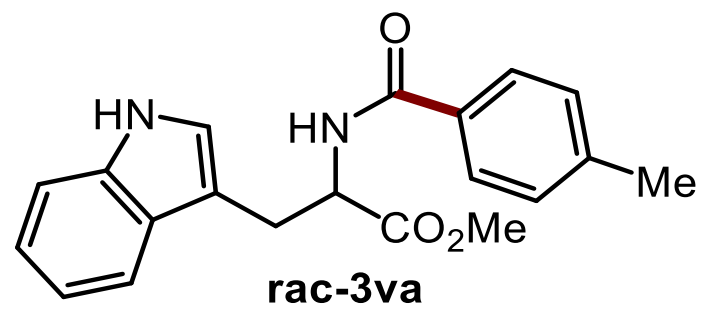

Compound name (rac-3va): Synthesized according to general procedure $\mathbf{B}$ using racemic tryptophan methyl ester hydrochloride $(0.127 \mathrm{~g}, 0.500 \mathrm{mmol}), \mathrm{Et}_{3} \mathrm{~N}(0.168 \mathrm{~mL}, 1.20 \mathrm{mmol})$, and $p$-toluoyl chloride $(0.0730 \mathrm{~mL}, 0.550 \mathrm{mmol})$, and $\mathrm{CH}_{2} \mathrm{Cl}_{2}(1.6 \mathrm{~mL})$. The reaction reached completion within 1 hour. The desired product was purified by column chromatography $\left(90 \% \mathrm{CH}_{2} \mathrm{Cl}_{2} / \mathrm{EtOAc}\right)$ yielding an amorphous white solid ( $0.106 \mathrm{~g}, 63 \%$ yield).<smiles>COC(=O)[C@H](Cc1c[nH]c2ccccc12)NC(=O)n1cc(C[C@H](NC(=O)c2ccc(C)cc2)C(C)=O)c2ccccc21</smiles>

methyl 3-(1-((3-(1H-indol-3-yl)-1-methoxy-1-oxopropan-2-yl)carbamoyl)-1H-indol-3-yl)-2-(4methylbenzamido)propanoate (3vva): Synthesized according to general procedure $\mathbf{D}$ using masked isocyanate $1 \mathbf{v}(0.203 \mathrm{~g}, 0.600 \mathrm{mmol})$, organoboron reagent $2 \mathrm{a}\left(0.244 \mathrm{~g}, 1.80 \mathrm{mmol}^{2}, \mathrm{Et}_{3} \mathrm{~N}(0.0836 \mathrm{~mL}\right.$, $0.600 \mathrm{mmol})$, and $[\mathrm{Rh}(\mathrm{OH})(\mathrm{cod})]_{2}(6.84 \mathrm{mg}, 0.0150 \mathrm{mmol})$. The reaction reached completion within 6 hours. The desired product was purified by column chromatography $\left(20 \% \mathrm{EtOAc} / \mathrm{CH}_{2} \mathrm{Cl}_{2}\right)$ yielding an offwhite foam ( $0.0416 \mathrm{~g}, 24 \%$ yield). TLC RF $=0.39$ in $20 \% \mathrm{EtOAC} / \mathrm{CH}_{2} \mathrm{Cl}_{2} .{ }^{1} \mathrm{H}-\mathrm{NMR}\left(300 \mathrm{MHz}, \mathrm{CDCl}_{3}\right): \delta 2.34$ $(3 \mathrm{H}, \mathrm{s}), 3.25-3.45(4 \mathrm{H}, \mathrm{m}), 3.61(3 \mathrm{H}, \mathrm{s}), 3.71(3 \mathrm{H}, \mathrm{s}), 4.93-5.00(1 \mathrm{H}, \mathrm{m}), 5.05-5.11(1 \mathrm{H}, \mathrm{m}), 6.09(1 \mathrm{H}, \mathrm{d}$, $J=7.5 \mathrm{~Hz}), 6.69(1 \mathrm{H}, \mathrm{d}, J=7.7 \mathrm{~Hz}), 6.97(1 \mathrm{H}, \mathrm{d}, J=2.4 \mathrm{~Hz}), 7.03-7.31(8 \mathrm{H}, \mathrm{m}), 7.44-7.58(4 \mathrm{H}, \mathrm{m}), 7.89(1 \mathrm{H}, \mathrm{d}$, $J=7.9 \mathrm{~Hz}), 8.31(1 \mathrm{H}, \mathrm{s}, \mathrm{br}) .{ }^{13} \mathrm{C}-\mathrm{NMR}(100 \mathrm{MHz}, \mathrm{CDCl}): \delta 21.5\left(\mathrm{CH}_{3}\right), 27.5\left(\mathrm{CH}_{2}\right), 52.5\left(\mathrm{CH}_{3}\right), 52.6\left(\mathrm{CH}_{3}\right), 53.0$ $(\mathrm{CH}), 54.1(\mathrm{CH}), 109.4(\mathrm{C}), 111.5(\mathrm{CH}), 114.4(\mathrm{CH}), 115.2(\mathrm{CH}), 118.4(\mathrm{C}), 119.1(\mathrm{CH}), 119.8(\mathrm{CH}), 122.3$ $(\mathrm{CH}), 122.3(\mathrm{CH}), 122.4(\mathrm{CH}), 123.1(\mathrm{CH}), 124.7(\mathrm{CH}), 127.1(\mathrm{CH}), 127.4(\mathrm{C}), 129.3(\mathrm{CH}), 130.1(\mathrm{C}), 130.8$ (C), 135.3 (C), 136.3 (C), 142.4 (C), 151.3 (C), 167.2 (C), 172.3 (C), 172.4 (C). IR (film): 3326, 1735, 1690, $1643 \mathrm{~cm}^{-1}$. HRMS (ESI): Exact mass calcd for $\mathrm{C}_{33} \mathrm{H}_{32} \mathrm{~N}_{4} \mathrm{O}_{6} \mathrm{Na}[\mathrm{M}+\mathrm{Na}]^{+}: 603.2219$. Found: 603.2200. [ $\left.\alpha\right]^{2{ }^{20}{ }^{-}}$ $36.3^{\circ}$ (c 1.34, $\left.\mathrm{MeCN}\right)$. 


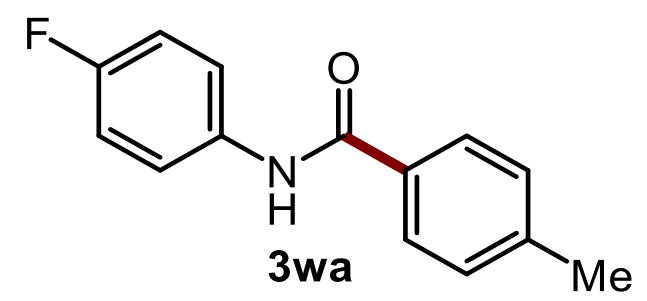

$\mathbf{N}$-(4-fluorophenyl)-4-methylbenzamide (3va): Synthesized according to general procedure $\mathbf{C}$ using masked isocyanate $1 \mathbf{w}(0.138 \mathrm{~g}, 0.600 \mathrm{mmol})$, boroxine $2 \mathrm{a}(0.106 \mathrm{~g}, 0.300 \mathrm{mmol}), \mathrm{Et}_{3} \mathrm{~N}(0.0836 \mathrm{~mL}$, $0.600 \mathrm{mmol})$, and $[\mathrm{Rh}(\mathrm{OH})(\mathrm{cod})]_{2}\left(2.73 \mathrm{mg}, 6.00 \times 10^{-3} \mathrm{mmol}\right)$. The reaction reached completion within 16 hours. The crude mixture was dry loaded onto silica gel (acetone) and purified by column chromatography ( $75 \% \mathrm{CH}_{2} \mathrm{Cl}_{2}$ /hexanes) white solid ( $0.107 \mathrm{~g}$, $78 \%$ yield). $\mathrm{TLC} \mathrm{R}_{\mathrm{F}}=0.18$ in $75 \% \mathrm{CH}_{2} \mathrm{Cl}_{2}$ / hexanes. ${ }^{1} \mathrm{H}-\mathrm{NMR}\left(300 \mathrm{MHz}, \mathrm{DMSO}-d_{6}\right): \delta 2.37(3 \mathrm{H}, \mathrm{s}), 7.17(2 \mathrm{H}, \mathrm{t}, J=8.9 \mathrm{~Hz}), 7.32(2 \mathrm{H}, \mathrm{d}, J=8.0 \mathrm{~Hz}), 7.76-$ $7.82(1 \mathrm{H}, \mathrm{m}), 7.87(2 \mathrm{H}, \mathrm{d}, \mathrm{J}=8.2 \mathrm{~Hz}), 10.22(1 \mathrm{H}, \mathrm{s}) .{ }^{13} \mathrm{C}-\mathrm{NMR}\left(75 \mathrm{MHz}, \mathrm{DMSO}-d_{6}\right): \delta 21.4\left(\mathrm{CH}_{3}\right), 115.5(\mathrm{CH}$, d, J=22.2 Hz), $122.6(\mathrm{CH}, \mathrm{d}, \mathrm{J}=7.82 \mathrm{~Hz}), 128.1(\mathrm{CH}), 129.3(\mathrm{CH}), 132.3(\mathrm{C}), 136.0(\mathrm{C}, \mathrm{d}, J=2.57 \mathrm{~Hz}), 142.0$ (C), 158.7 (C, d, J=240.0 Hz), 165.7 (C). Data consistent with literature. ${ }^{12}$

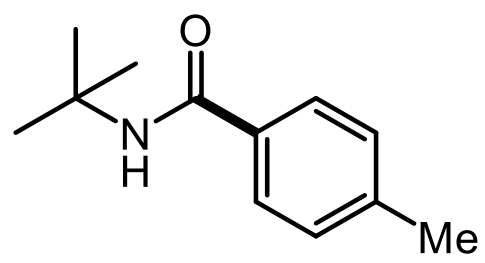

$\mathbf{N}$-(4-fluorophenyl)-4-methylbenzamide (3xa): Synthesized according to general procedure $\mathbf{C}$ using masked isocyanate $1 \times 1 \times(0.116 \mathrm{~g}, 0.600 \mathrm{mmol})$, boroxine $2 \mathrm{a}(0.109 \mathrm{~g}, 0.300 \mathrm{mmol}), \mathrm{Et}_{3} \mathrm{~N}(0.0836 \mathrm{~mL}, 0.600$ $\mathrm{mmol})$, and $[\mathrm{Rh}(\mathrm{OH})(\mathrm{cod})]_{2}\left(2.73 \mathrm{mg}, 6.00 \times 10^{-3} \mathrm{mmol}\right)$ at $100{ }^{\circ} \mathrm{C}$. The reaction reached completion within 18 hours. The desired product was purified by column chromatography (10\% EtOAc/Hexanes) yielding an amorphous white solid $\left(0.0971 \mathrm{~g}, 84 \%\right.$ yield). TLC RF $=0.47$ in $20 \%$ EtOAc/Hexanes. ${ }^{1} \mathrm{H}-\mathrm{NMR}(500 \mathrm{MHz}$, $\left.\mathrm{CDCl}_{3}\right) \delta 7.63-7.58(\mathrm{~m}, 2 \mathrm{H}), 7.23-7.18(\mathrm{~m}, 2 \mathrm{H}), 5.95-5.83 .(\mathrm{s}, 1 \mathrm{H}), 2.38(\mathrm{~s}, 3 \mathrm{H}), 1.46(\mathrm{~s}, 9 \mathrm{H}) .{ }^{13} \mathrm{C}-\mathrm{NMR}$ $\left(126 \mathrm{MHz}, \mathrm{CDCl}_{3}\right) \delta 167.0(\mathrm{C}), 141.5(\mathrm{C}), 133.2(\mathrm{C}), 129.2(\mathrm{CH}), 126.8(\mathrm{CH}), 51.6(\mathrm{C}), 29.1\left(\mathrm{CH}_{3}\right), 21.5\left(\mathrm{CH}_{3}\right)$. Data consistent with literature. ${ }^{24}$ 


\section{9-Compatibility of Aliphatic Amines (Table S11-S12)}

The compatibility of aliphatic amines, known to add to isocyanates at a rate 200-300 times greater than anilines, was tested through the addition of morpholine (1.1 equiv.) to the mixture described general procedure D. A variety of conditions using phenol as a blocking group were tested resulting in limited success (table S11). The morpholine urea (5aa) adduct was consistently formed as the dominant product.

Table S11. Synthesis of substrate 3aa in the presence of morpholine

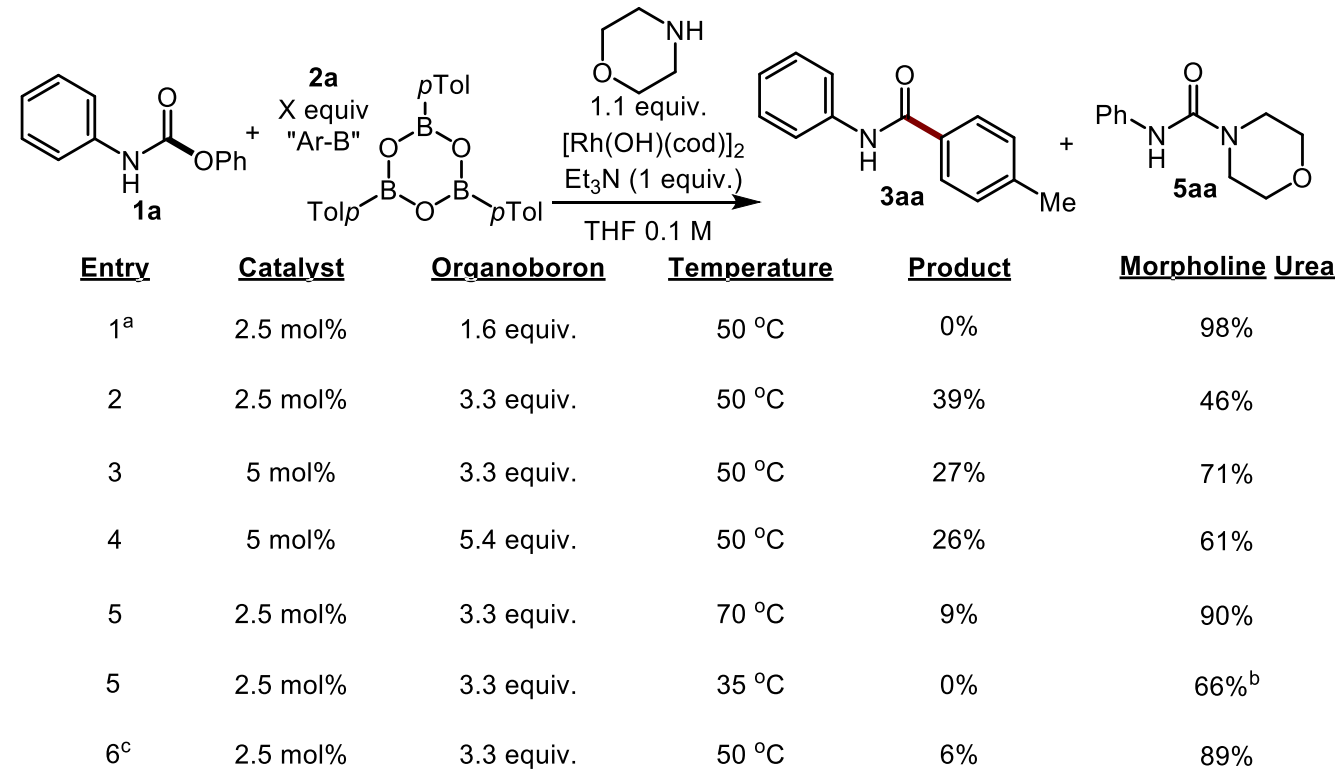

${ }^{\mathrm{a}} 0.5 \mathrm{M}$ THF. ${ }^{\mathrm{b}}$ Reaction did not reach completion. ${ }^{\mathrm{c}} 0.2$ equiv. of $\mathrm{Et}_{3} \mathrm{~N}$

We hypothesized using a different blocking group could help tune the system resulting in slower formation of 5aa. Thus efforts continued using diisopropylamine blocked isocyanate $\mathbf{4 a}$, which generates the requisite isocyanates by steric decompression. ${ }^{25}$ Pleasingly, a slight change of the reaction conditions with blocked isocyanate 4a provided the desired product with minimal urea by-product (Scheme S1). The desired product was also obtained in the presence of dibenzylamine and benzylamine.

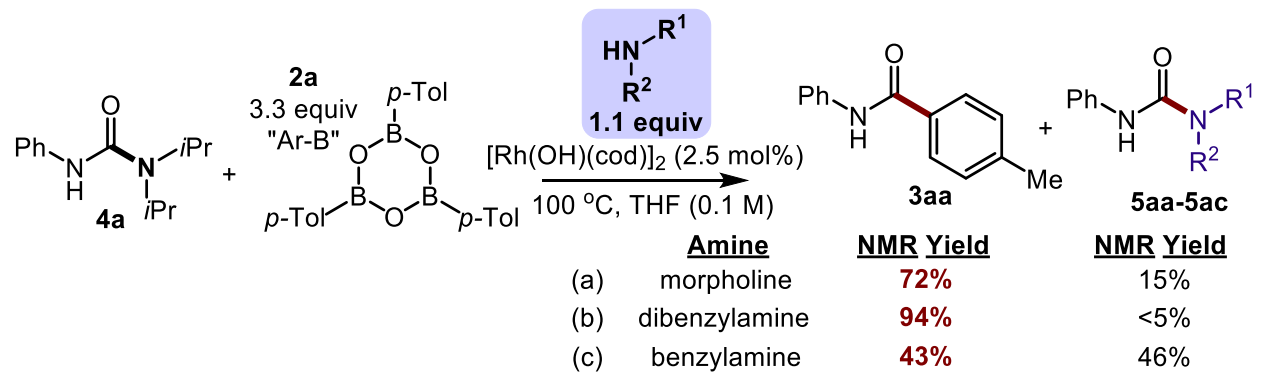

Scheme S1. Aliphatic amine compatibility experiments 
Interestingly, control reactions probing the reversibility of the urea formation showed the potential for the morpholine urea (5aa) adduct to act as blocking group, providing the desired product in high yield when used as the starting material. In contrast, all other ureas tested, including both dibenzyl amine (5ab) and benzylamine (5ac) derived urea, showed minimal reversibility. This suggests the formation of product 3aa in the presence of such nucleophiles is in fact a kinetic phenomenon. Overall, this achievement highlights the versatility of the blocked isocyanate strategy: under appropriate conditions the catalytically generated arylrhodium intermediate B (Figure 1C) can outcompete excellent nucleophiles present in stoichiometric amounts. The vast array of blocking groups, which span several different deblocking methods and can be fine-tuned, may serve to accelerate the implementation of a blocked isocyanate strategy in other metal-catalyzed transformations.

Table S12. Investigation of ureas 5aa-5ae as isocyanate precursors

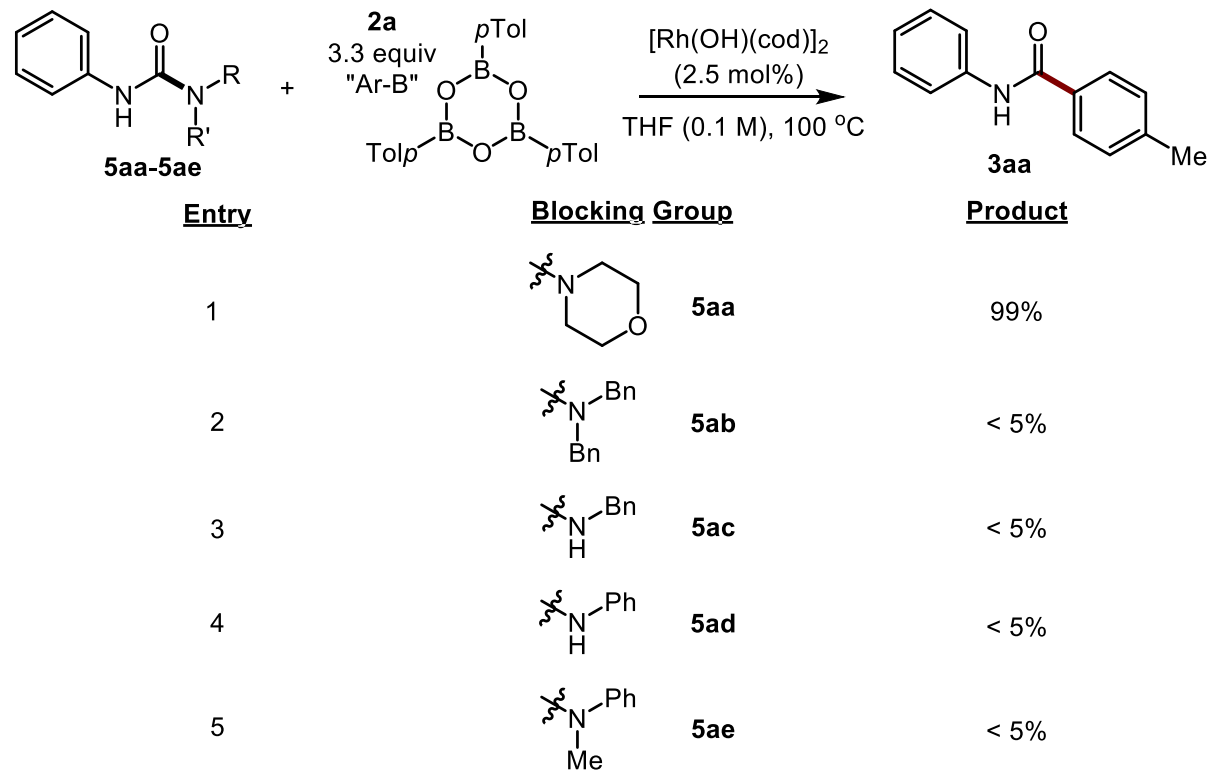

\section{0-Variable Time Normalization Plots}

The graphical method developed by Burés ${ }^{26}$ was used to determine the reaction orders from the concentration profiles. This method uses a variable normalization of the time scale to enable the visual comparison of entire concentration profiles, allowing for the order in each component of the reaction to be determined.<smiles>CCN(CC)C(=O)OCc1ccc(NC(=O)c2ccc(C)cc2)cc1</smiles>

General procedure $C$ and $D$ were followed using an NMR tube in place of a microwave vial on 0.300 $\mathrm{mmol}$ scale and $0.0600 \mathrm{mmol}$ scale respectively. Organoboron reagent $2 \mathrm{a}$ was fully dehydrated to the 
boroxine before use. $\mathrm{PhCF}_{3}(0.100 \mathrm{~mL}, 0.0814 \mathrm{mmol})$ was used as an internal standard and the reaction was monitored by fluorine NMR at specified time points. The raw data obtained, and used in this section, is presented in section 10 .

The graphical method developed by Burés was used to plot [Product] against $\mathrm{t}$ [cat $]^{\alpha}$ to determine the order in catalyst. When ${ }^{\alpha}$ is the correct order in catalyst the traces will overlay.
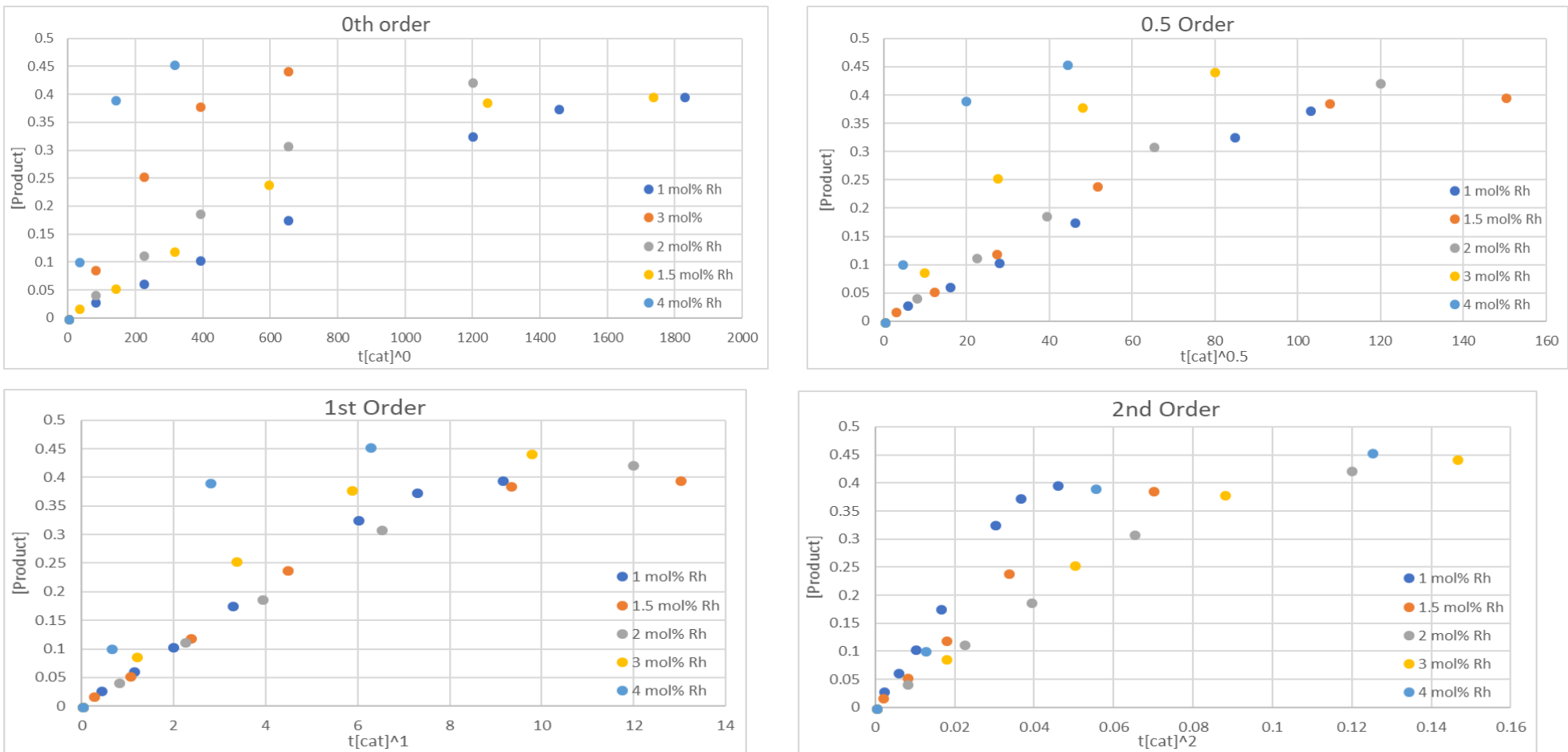

Figure S7. Variable time normalization plots for the determination of catalyst order under conditions described in general procedure $\mathrm{C}$ (low $[\mathrm{RBO}]_{3}$, high [THF])

The lack of overlap in any of the 4 graphs is Figure $\mathbf{S 7}$ suggests a possible change in rate determining step at different catalyst loadings under these conditions. This was explored by plotting the catalyst loadings 1-2 mol\% and 2-4 mol\% separately seen below (Figures S8-S9). 

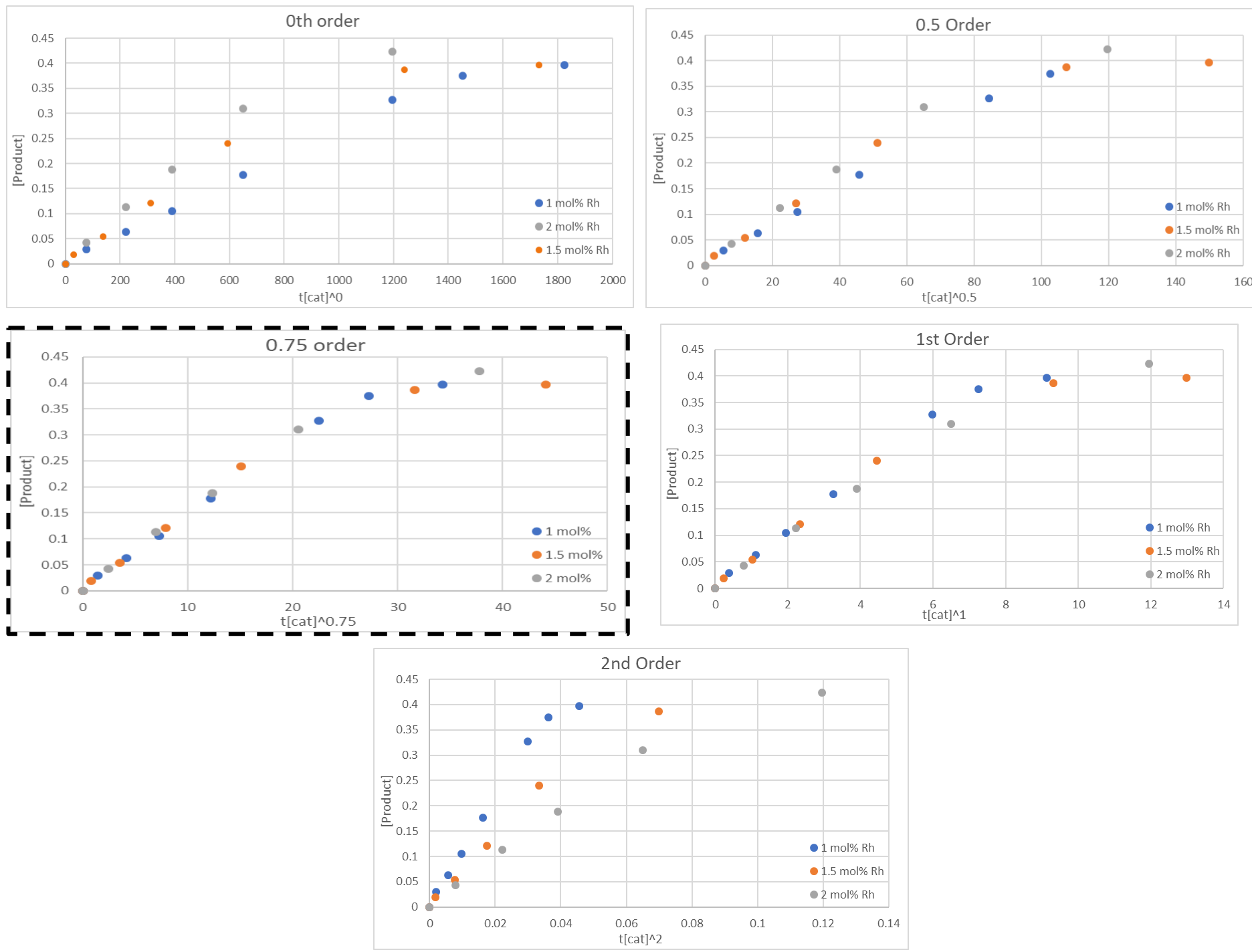

Figure S8. Variable time normalization plots for the determination of catalyst order with loadings of 1-2 mol\% under conditions described in general procedure $\mathrm{C}$ (low $[\mathrm{RBO}]_{3}$, high [THF])

The order in catalyst under these conditions was found to be 0.75 by analyzing plots in Figure S8. This suggest that the catalyst exist partially as a dimeric species under these conditions. 

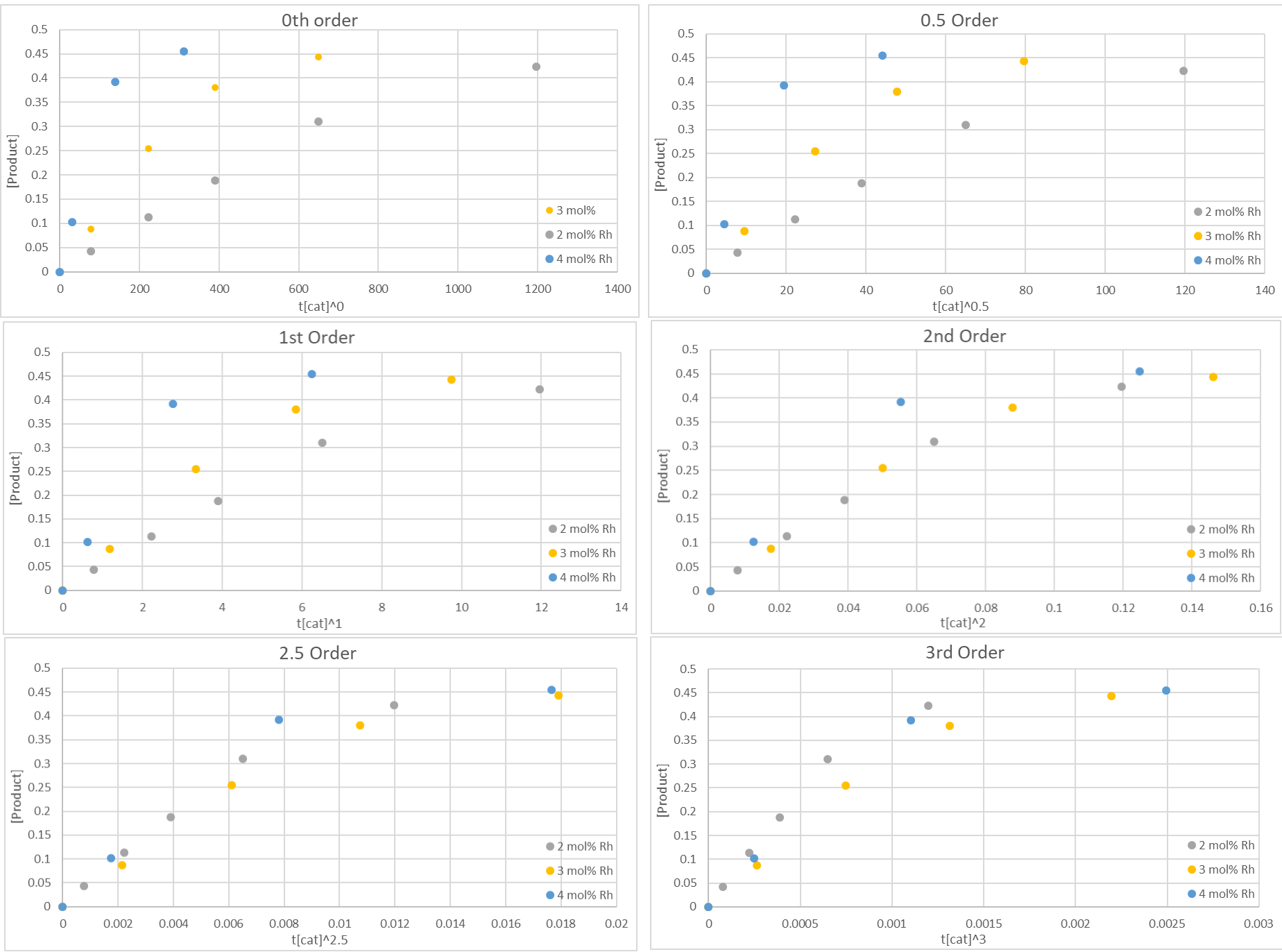

Figure S9. Variable time normalization plots for the determination of catalyst order with loadings of 2-4 mol\% under conditions described in general procedure $\mathrm{C}$ (low $[\mathrm{RBO}]_{3}$, high [THF])

The lack of an overlap on all the graphs in Figure $\mathbf{S 9}$ led us to graph 2-3/3-4 mol\% separately seen in Figure S10-11. 

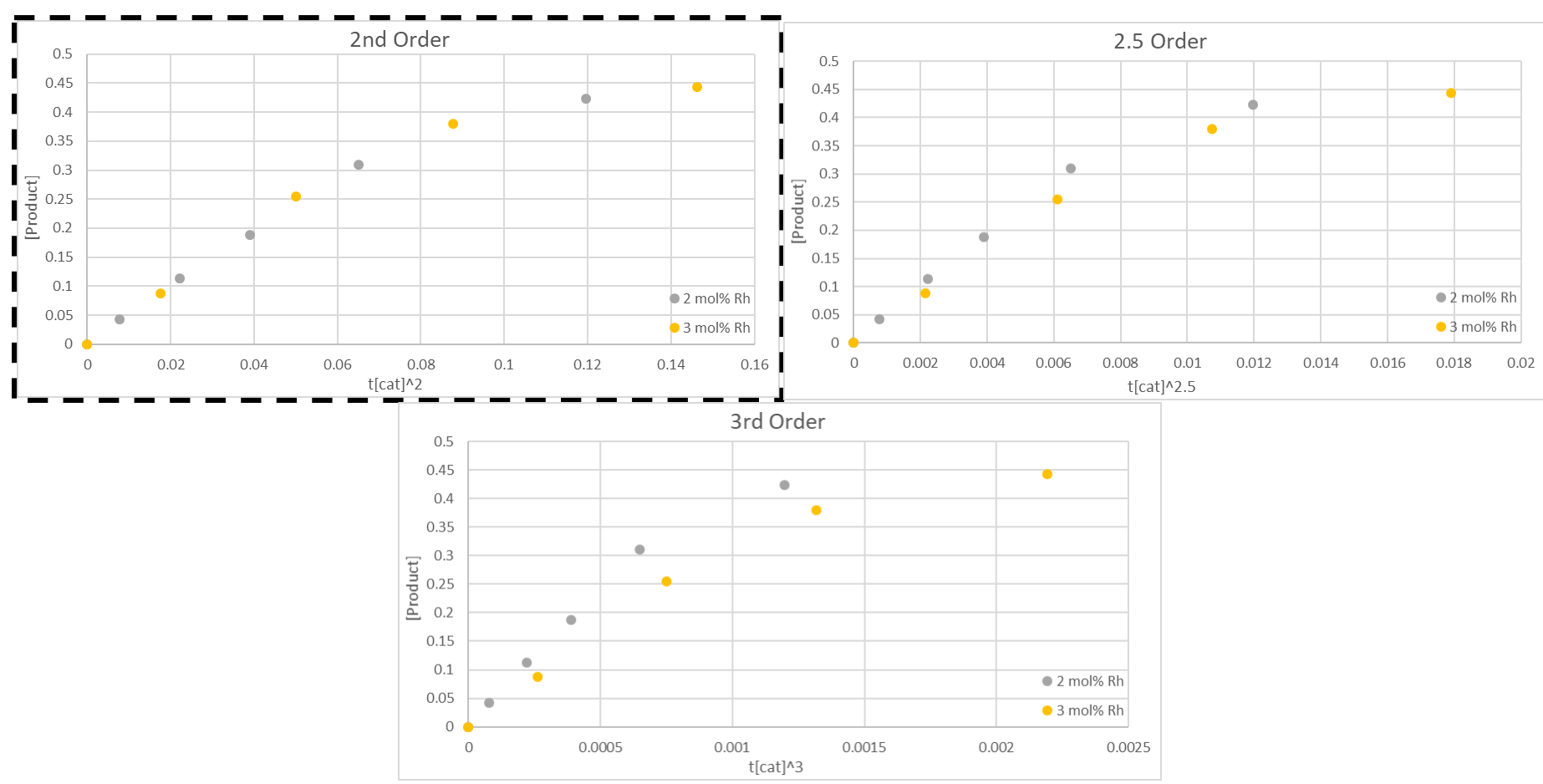

Figure S10. Variable time normalization plots for the determination of catalyst order with loadings of 2-3 mol\% under conditions described in general procedure $\mathrm{C}$ (low [RBO $]_{3}$, high [THF])

See below for discussion of the results seen above in Figure S10. 

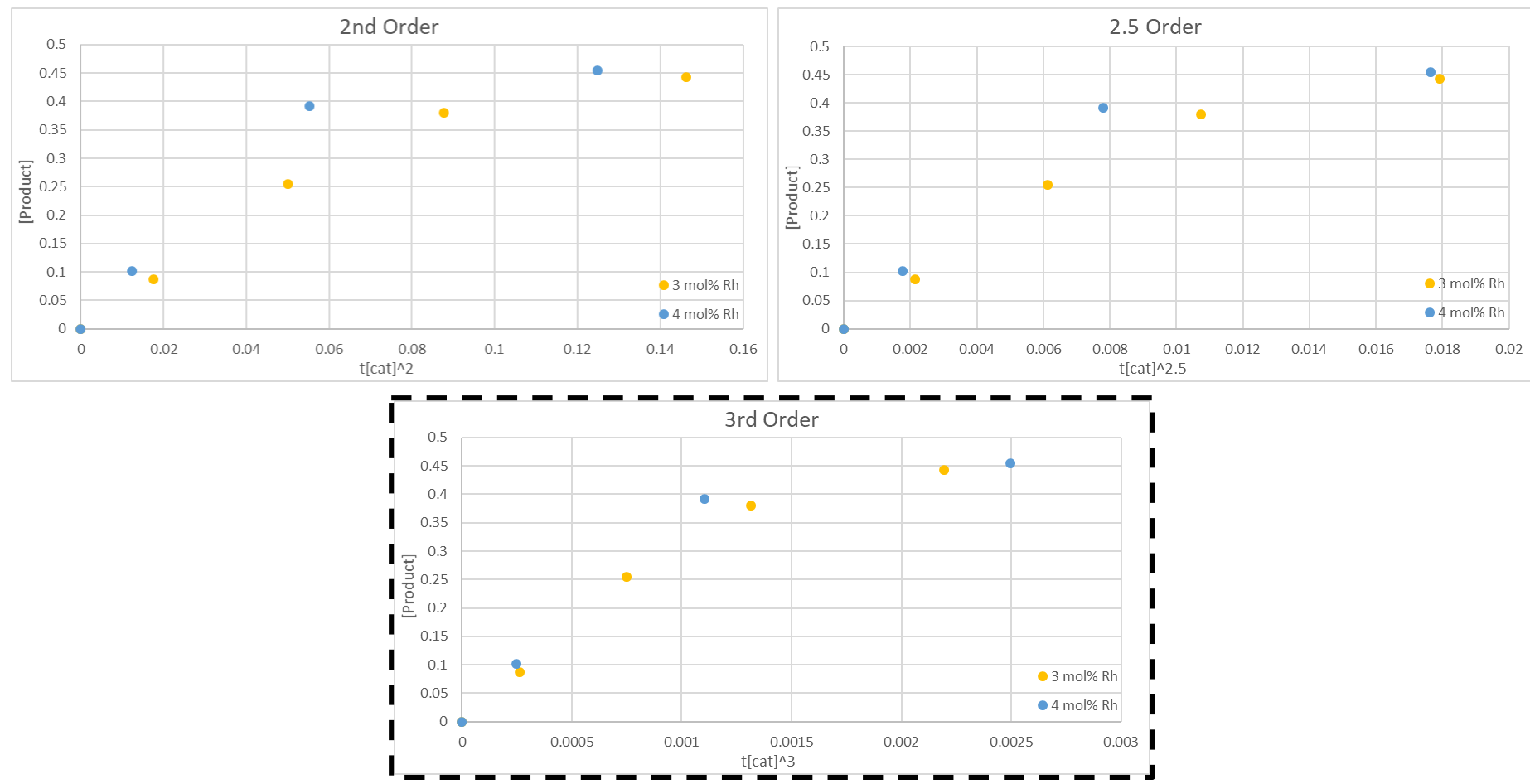

Figure S11. Variable time normalization plots for the determination of catalyst order with loadings of 3-4 mol\% under conditions described in general procedure $\mathrm{C}$ (low $[\mathrm{RBO}]_{3}$, high [THF])

The order in catalyst was observed to be $2^{\text {nd }}$ order between $2 / 3$ mol\% Rh (Figure S10), while a $3^{\text {rd }}$ order catalyst order is observed between $3 / 4$ mol\% Rh (Figure S11). The existence of trimeric rhodium species have been reported for the addition of boronic acids onto enone electrophile though not as the active catalytic species. ${ }^{27}$ Given this precedent, we postulate the $2^{\text {nd }}$ order reaction observed between $2 / 3$ mol\% Rh is in fact an artifact of taking measurements at catalyst loadings where both pathways (an active rhodium monomer and an active rhodium trimer) are operating. More concisely, below 2 mol\% $\mathrm{Rh}$, the active rhodium species is a monomer, while above $3 \mathrm{~mol} \%$, the active rhodium catalyst is a trimeric species. While this preliminary result is for higher catalyst concentrations than used in Tables 2 , and thus outside of the scope of the current study, it is currently under further investigation. 

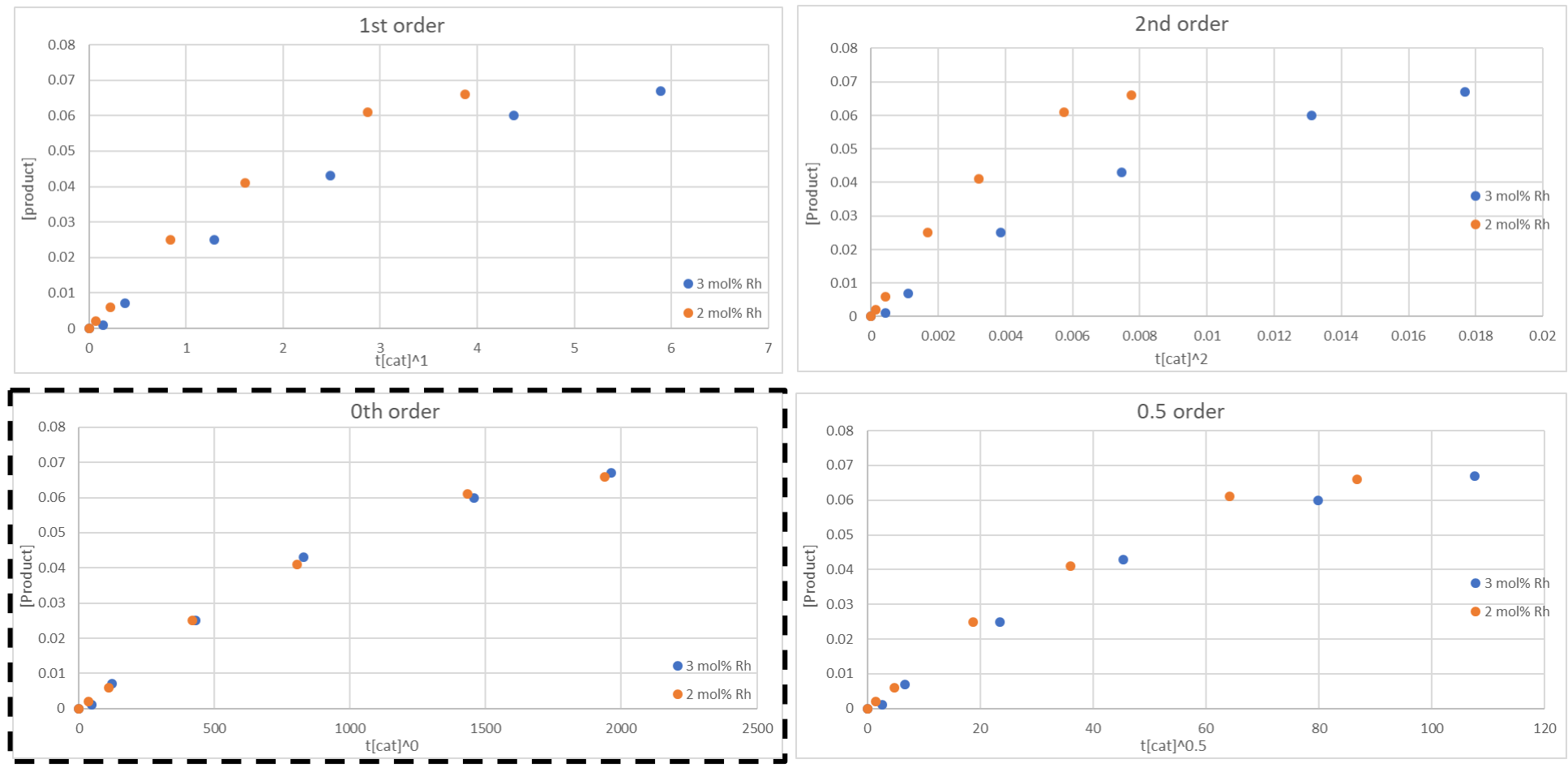

Figure S12. Variable time normalization plots for the determination of catalyst order under conditions described in general procedure $\mathrm{D}$ (high $[\mathrm{RBO}]_{3}$, low $[\mathrm{THF}]$ )

In contrast to the above cases, the order in catalyst under the conditions optimized for the amphoteric / ambident isocyanate precursors shown in Table $2 \mathrm{c}$ was found to be 0 by analyzing plots in Figure S12. This suggest that the catalyst is not involved in the rate determining step at lower concentrations. The lack of involvement of the catalyst in the rate determining step under these conditions supports the existence of an isocyanate intermediate as opposed to direct $\mathrm{C}-\mathrm{O}$ bond activation ${ }^{28}$ or direct addition onto the carbamate ${ }^{29}$ where catalyst involvement would be present in all steps of the transformation. 
The graphical method developed by Burés was used to plot [Product] against the variable time scale normalized in carbamate $\mathbf{1} \mathbf{w}\left(\Sigma[\text { carbamate }]^{\alpha} \Delta \mathrm{t}\right)$. When $\alpha$ is the correct order in $\mathbf{1} \mathbf{w}$ the traces will overlay.

$$
\int_{t=0}^{t=n}[\mathrm{~A}]^{\alpha} \mathrm{d} t=\sum_{i=1}^{n}\left(\frac{[\mathrm{A}]_{i}+[\mathrm{A}]_{i-1}}{2}\right)^{\alpha}\left(t_{i}-t_{i-1}\right)
$$
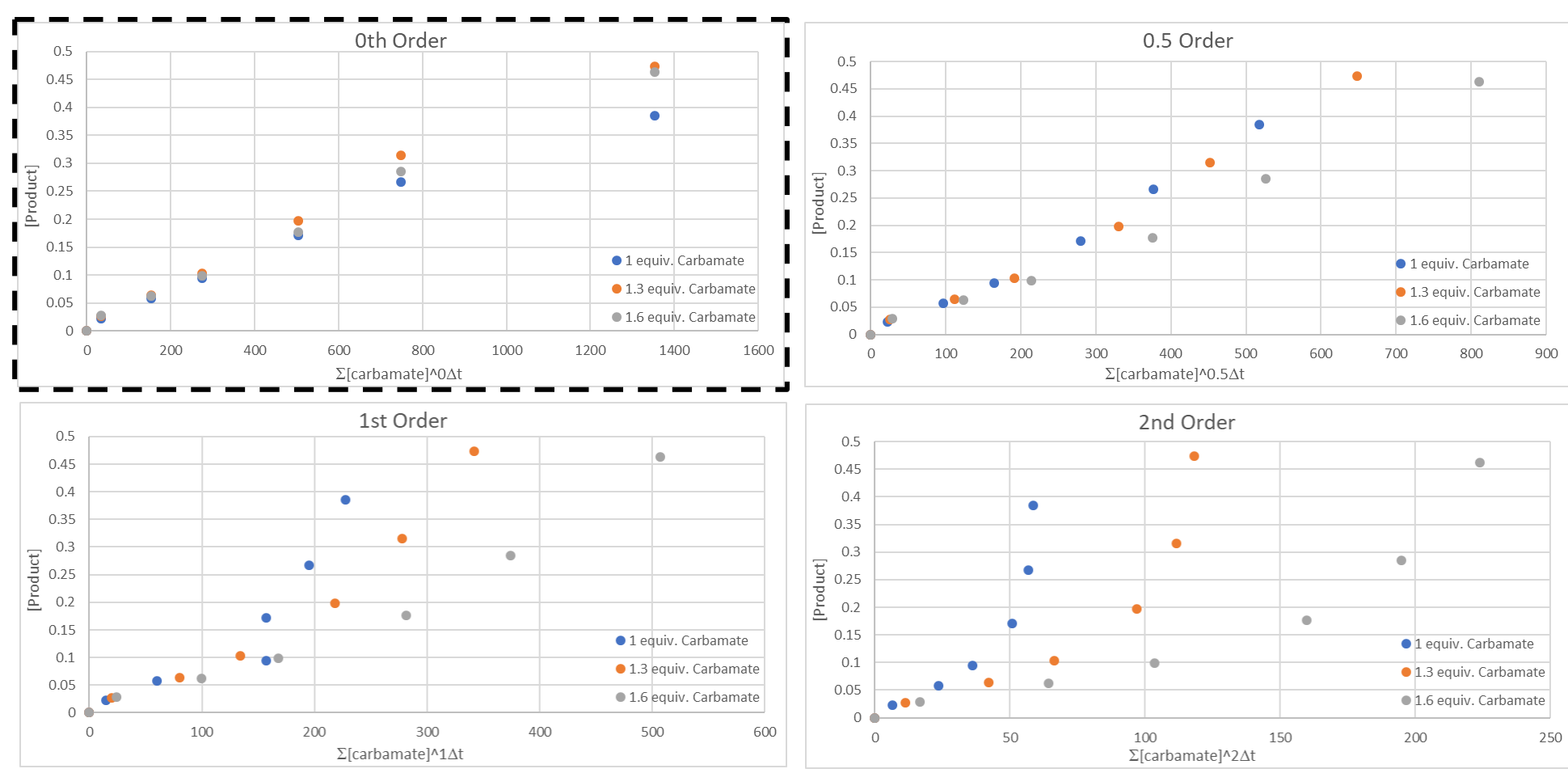

Figure S13: Variable time normalization plots for the determination of order in $\mathbf{1} \mathbf{w}$ under conditions described in general procedure $\mathrm{C}\left(\right.$ low $[\mathrm{RBO}]_{3}$, high $[\mathrm{THF}]$ ) with $1 \mathrm{~mol} \%[\mathrm{Rh}(\mathrm{OH})(\mathrm{cod})]_{2}$

The order in $\mathbf{1 w}$ under the conditions used in Tables $2 \mathrm{a}$ and $2 \mathrm{~b}$ was found to be 0 by analyzing plots in Figure S13. This result coupled with the 0.75 order in catalyst (Figure S8) suggests a rate determining transmetallation, with the existence of a dimeric rhodium specie under such conditions. 

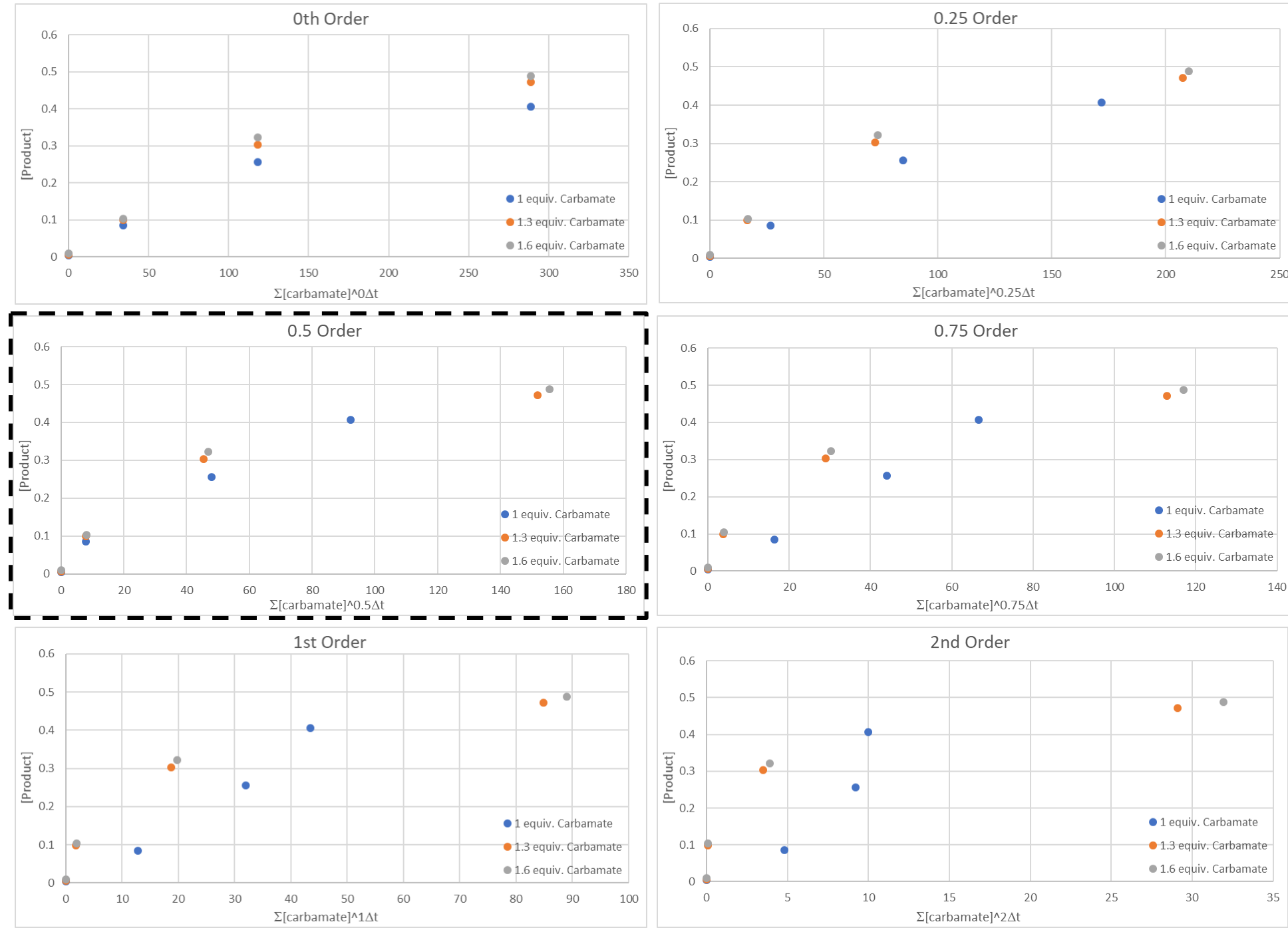

Figure S14: Variable time normalization plots for the determination of order in $\mathbf{1} \mathbf{w}$ under conditions described in general procedure $\mathrm{C}\left(\right.$ low $[\mathrm{RBO}]_{3}$, high $\left.[\mathrm{THF}]\right)$ with $3 \mathrm{~mol} \%[\mathrm{Rh}(\mathrm{OH})(\mathrm{cod})]_{2}$

The order in $\mathbf{1 w}$ under the conditions used in Table $2 \mathrm{a}$ and $\mathrm{b}$ at high catalyst loading was found to be 0.5 by analyzing plots in Figure S14. This result suggests the existence of $\mathbf{1 w}$ as a dimer, possibly due to hydrogen-bonding. ${ }^{30}$ This result coupled with the higher order in catalyst (figure S10/S11) suggests the possibility of a rate determining insertion. The exact mechanistic intricacies of this particular kinetic regime are not yet fully understood and currently under further investigation. 

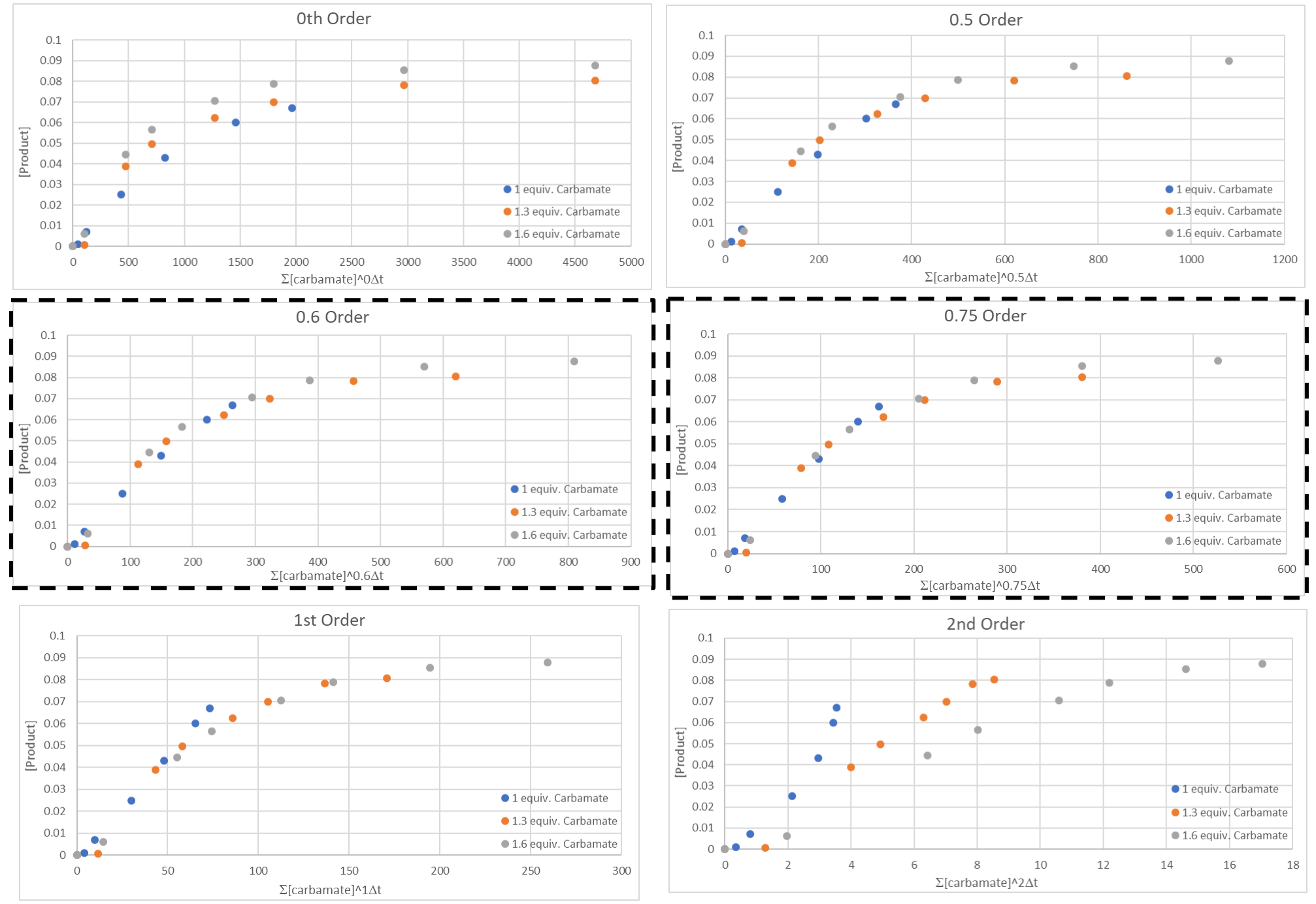

Figure S15: Variable time normalization plots for the determination of order in $\mathbf{1 w}$ under conditions described in general procedure $\mathrm{D}\left(\mathrm{high}[\mathrm{RBO}]_{3}\right.$, low $\left.[\mathrm{THF}]\right)$ with $3 \mathrm{~mol} \%[\mathrm{Rh}(\mathrm{OH})(\mathrm{cod})]_{2}$

The order of $1 \mathbf{w}$ under the conditions used in Table $2 c$ was found to be between $0.6-0.75$ order by analyzing plots in Figure S15. This suggest the existence of $\mathbf{1 w}$ as a dimeric species under these conditions. This coupled with the $0^{\text {th }}$ order in catalyst (Figure S12) further supports a rate determining isocyanate unmasking under the conditions described in general procedure $\mathrm{D}$. 
Similarly, the graphical method developed by Burés was used to plot [Product] against t[Base] ${ }^{\alpha}$ to determine the order in Base. When $\alpha$ is the correct order in base the traces will overlay.
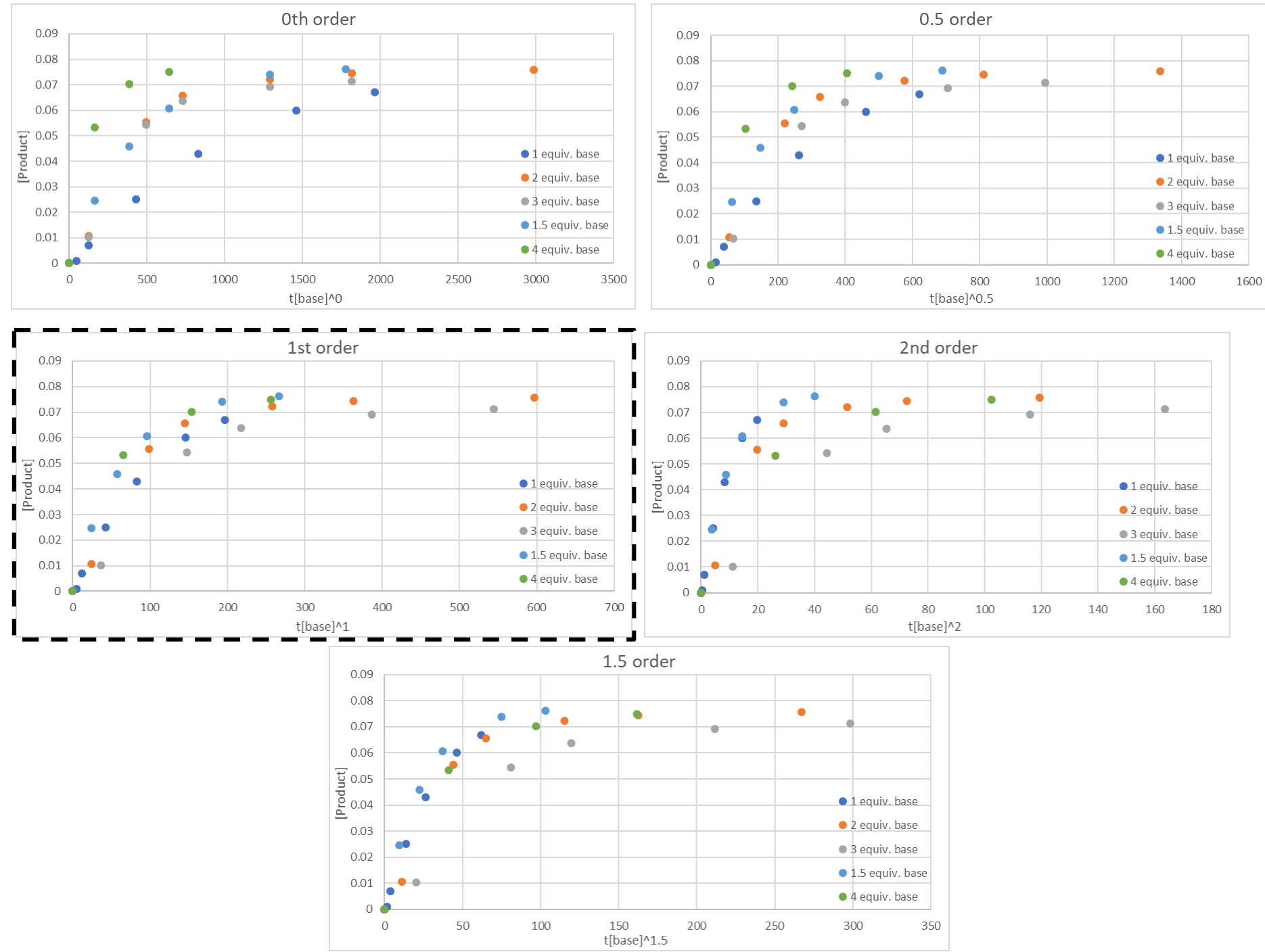

Figure S16: Variable time normalization plots for the determination of order in $\mathrm{Et}_{3} \mathrm{~N}$ under conditions described in general procedure $D\left(\right.$ high $[\mathrm{RBO}]_{3}$, low $\left.[\mathrm{THF}]\right)$ with $3 \mathrm{~mol} \%[\mathrm{Rh}(\mathrm{OH})(\mathrm{cod})]_{2}$

The order of $\mathrm{Et}_{3} \mathrm{~N}$ under the conditions used in Table $2 \mathrm{c}$ was found to be 1 by analyzing plots in Figure S16. This result further supports deblocking as the rate determining step under conditions used in Table $2 \mathrm{c}$, specifically a base mediated isocyanate unmasking mentioned in the communication. 


\section{1-Raw data for Figures S5-S14 (Tables S13-S18)}

Table S13. Raw data for the determination of order in catalyst under conditions of general procedure C. The reaction was run with $1 \mathrm{~mol} \%, 1.5 \mathrm{~mol} \%, 2 \mathrm{~mol} \%, 3 \mathrm{~mol} \%, 4 \mathrm{~mol} \%[\mathrm{Rh}(\mathrm{OH})(\mathrm{Cod})]_{2}$

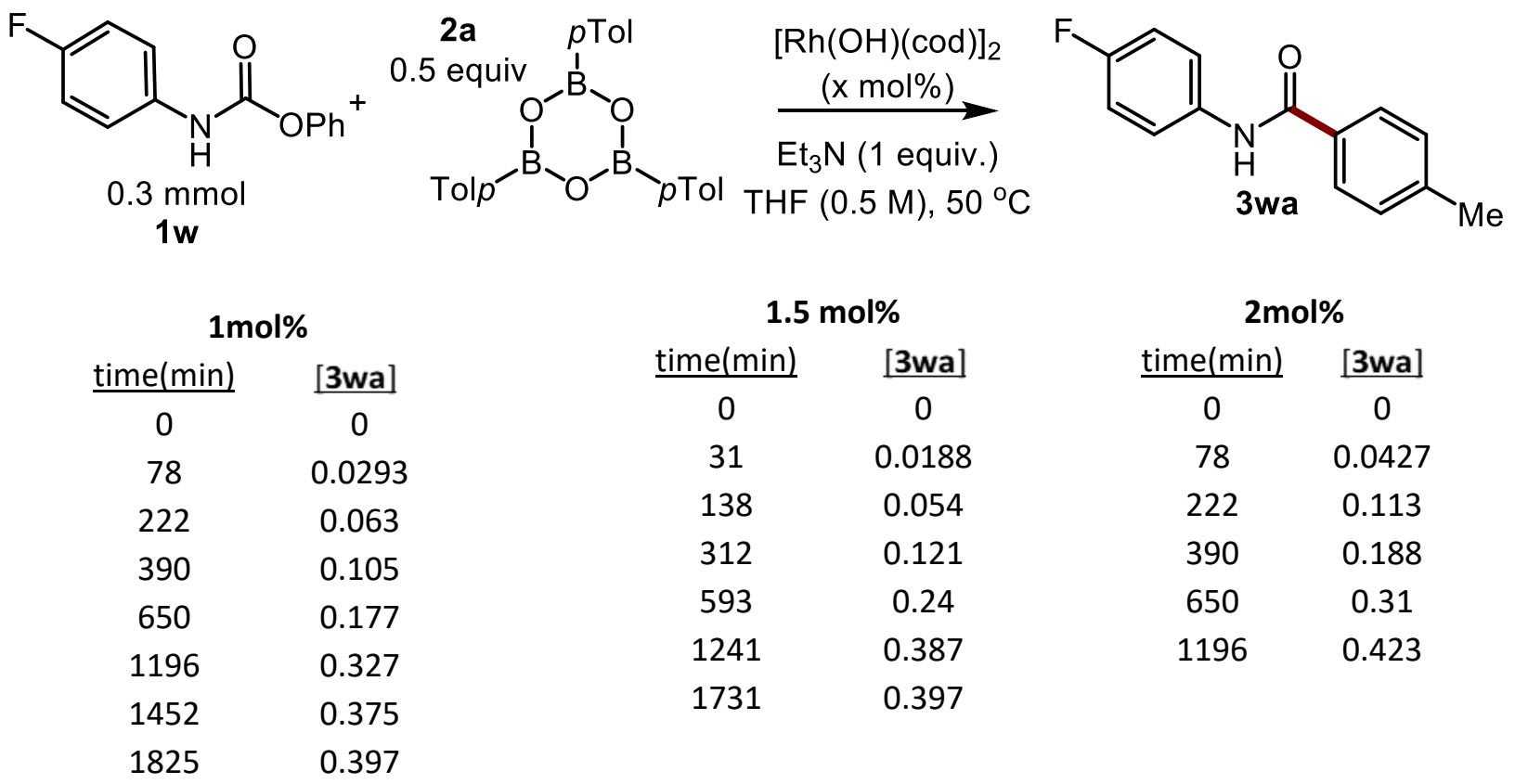

\begin{tabular}{ccccc}
\multicolumn{2}{c}{ 3mol\% } & \multicolumn{2}{c}{ 4mol\% } \\
time(min) & [3wa] & & time(min) & [3wa] \\
\cline { 2 - 4 } 0 & 0 & & 0 & 0 \\
78 & 0.0877 & & 31 & 0.102 \\
222 & 0.255 & & 138 & 0.392 \\
390 & 0.38 & 312 & 0.455 \\
650 & 0.443 & &
\end{tabular}


Table S14. Raw data for the determination of order in catalyst under conditions of general procedure D. The reaction was run with $2 \mathrm{~mol} \%$ and $3 \mathrm{~mol} \%[\mathrm{Rh}(\mathrm{OH})(\mathrm{cod})]_{2}$
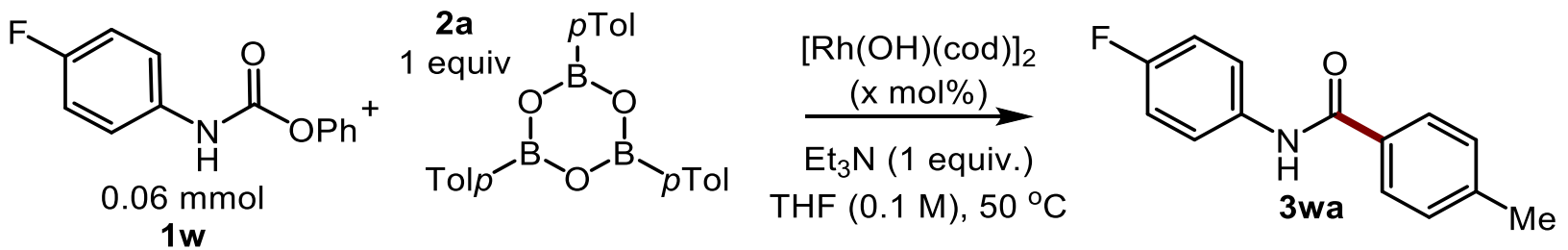

\begin{tabular}{cccc}
\multicolumn{2}{c}{ 2mol\% } & \multicolumn{2}{c}{ 3mol\% } \\
$\frac{\text { time }}{0}$ & {$[$ [3wa] } & time(min) & [3wa] \\
35 & 0 & 0 & 0 \\
109 & 0.002 & 48 & 0.001 \\
418 & 0.006 & 123 & 0.007 \\
804 & 0.041 & 430 & 0.025 \\
1434 & 0.061 & 828 & 0.043 \\
1939 & 0.066 & 1458 & 0.06 \\
& & 1963 & 0.067
\end{tabular}


Table S15. Raw data for the determination of order in $\mathbf{1} \mathbf{w}$ under conditions of general procedure $\mathrm{C}$ at low catalyst concentration. The reaction was run with $1,1.3$, and 1.6 equiv of $1 \mathbf{w}$ where 1 equiv $=0.3$ $\mathrm{mmol}$

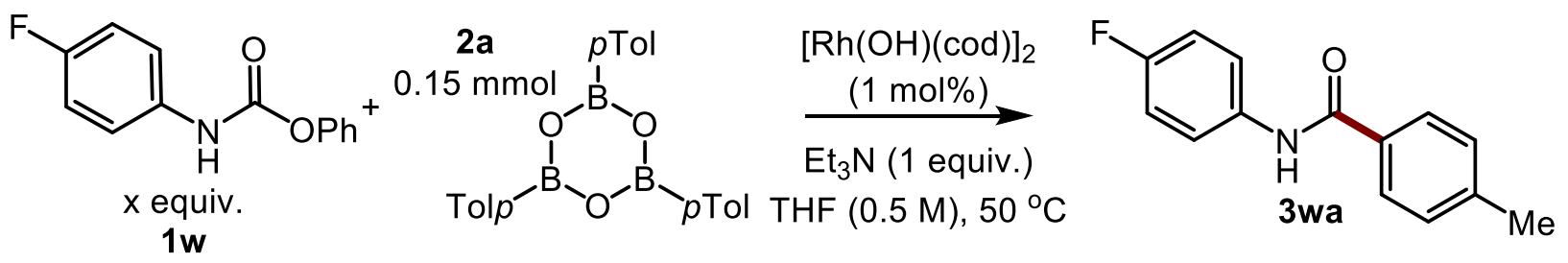

\begin{tabular}{ccc} 
time(min) & $\begin{array}{c}\text { 1 equiv. 1w } \\
{[\mathbf{1 w}]}\end{array}$ & {$[3 \mathbf{w a}]$} \\
\hline 0 & 0.4688 & 0 \\
34 & 0.4066 & 0.0228 \\
154 & 0.3485 & 0.0577 \\
274 & 0.2998 & 0.0944 \\
504 & 0.2036 & 0.1714 \\
749 & 0.1082 & 0.2666 \\
1354 & 0 & 0.385
\end{tabular}

\begin{tabular}{ccc}
\multicolumn{3}{c}{ 1.3 equiv. 1w } \\
\cline { 1 - 2 } time(min) & {$[\mathbf{1 w}]$} & {$[\mathbf{3 w a}]$} \\
0 & 0.6177 & 0 \\
34 & 0.5394 & 0.0271 \\
154 & 0.4747 & 0.0641 \\
274 & 0.4211 & 0.1035 \\
504 & 0.3084 & 0.1977 \\
749 & 0.1811 & 0.315 \\
1354 & 0.0292 & 0.4736
\end{tabular}

1.6 equiv. $1 w$

\begin{tabular}{|c|c|c|}
\hline time $(\min )$ & {$[1 w]$} & [3wa] \\
\hline 0 & 0.7391 & 0 \\
\hline 34 & 0.6629 & 0.0284 \\
\hline 154 & 0.5974 & 0.0627 \\
\hline 274 & 0.5445 & 0.0987 \\
\hline 504 & 0.444 & 0.1766 \\
\hline 749 & 0.3134 & 0.2853 \\
\hline 1354 & 0.1263 & 0.4627 \\
\hline
\end{tabular}


Table 16. Raw data for the determination of order in $\mathbf{1} \mathbf{w}$ under conditions of general procedure $\mathrm{C}$ at high catalyst concentration. The reaction was run with $1,1.3$, and 1.6 equiv of $1 \mathbf{w}$ where 1 equiv $=0.3 \mathrm{mmol}$
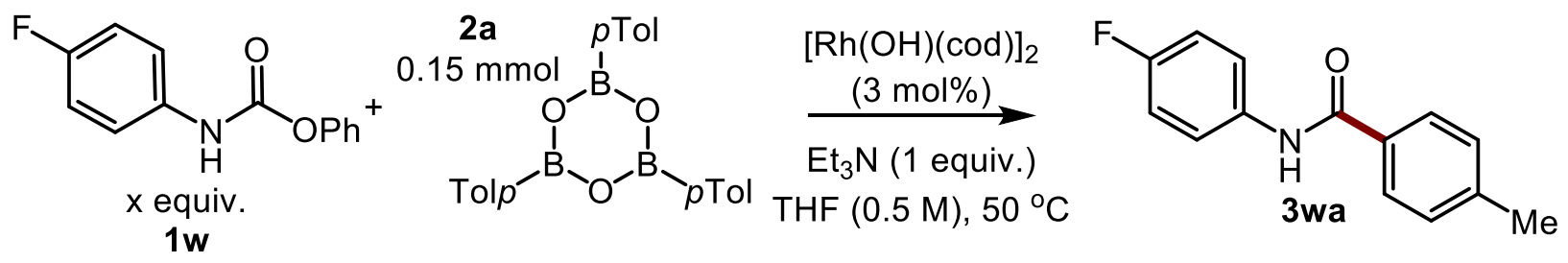

\begin{tabular}{ccc} 
& 1 equiv. 1w & \\
time $(\min )$ & {$[\mathbf{w}]$} & {$[\mathbf{3 w a}]$} \\
\hline 0 & 0.4317 & 0.004 \\
34 & 0.3219 & 0.0852 \\
118 & 0.1346 & 0.256 \\
289 & 0 & 0.4065
\end{tabular}

\begin{tabular}{ccc}
\multicolumn{3}{c}{ 1.3 equiv. 1w } \\
time(min) & $\underline{1 \mathbf{w}]}$ & {$[\mathbf{3 w a}]$} \\
\hline 0 & 0.5689 & 0.0065 \\
34 & 0.4332 & 0.0992 \\
118 & 0.2075 & 0.3026 \\
289 & 0.045 & 0.4715
\end{tabular}

1.6 equiv. $1 w$

$\begin{array}{cccc}\frac{\text { time }(\min )}{0} & & {[\mathbf{1 w}]} & \underline{[3 w a]} \\ 34 & & 0.6904 & 0.0092 \\ 118 & 0.5475 & 0.1038 \\ 289 & 0.2953 & 0.3222 \\ & 0.1164 & 0.4876\end{array}$


Table S17. Raw data for the determination of order in $\mathbf{1 w}$ under conditions of general procedure $D$ at 3 mol\% catalyst. The reaction was run with $1,1.3$, and 1.6 equiv of $1 \mathbf{w}$ where 1 equiv $=0.06 \mathrm{mmol}$
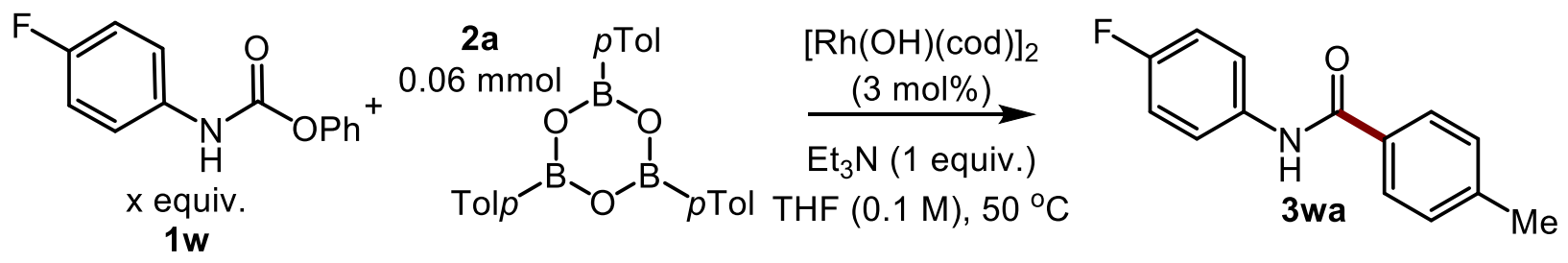

\begin{tabular}{|c|c|c|}
\hline time(min) & $\begin{array}{l}\text { equiv. } \\
\text { [3wa] }\end{array}$ & [1w] \\
\hline 0 & 0 & 0.084 \\
\hline 48 & 0.001 & 0.083 \\
\hline 123 & 0.007 & 0.076 \\
\hline 430 & 0.025 & 0.055 \\
\hline 828 & 0.043 & 0.036 \\
\hline 1458 & 0.06 & 0.019 \\
\hline 1963 & 0.067 & 0.012 \\
\hline
\end{tabular}

1.3 equiv. $1 \mathrm{w}$

$\begin{array}{cccc}\text { time }(\min ) & & {[\text { [wa }]} & {[\mathbf{1 w}]} \\ 0 & 0 & 0.114 \\ 107 & 0.000573 & 0.104 \\ 476 & 0.0388 & 0.068 \\ 711 & 0.0497 & 0.0573 \\ 1272 & 0.0623 & 0.0415 \\ 1800 & 0.07 & 0.0325 \\ 2970 & 0.0782 & 0.0212 \\ 4680 & 0.0805 & 0.0188\end{array}$

\begin{tabular}{|c|c|c|}
\hline \multicolumn{3}{|c|}{1.6 equiv. $1 \mathrm{w}$} \\
\hline time(min) & [3wa] & [1w] \\
\hline 0 & 0 & 0.14 \\
\hline 107 & 0.00617 & 0.131 \\
\hline 476 & 0.0445 & 0.089 \\
\hline 711 & 0.0565 & $0.075 \varepsilon$ \\
\hline 1272 & 0.0705 & 0.0595 \\
\hline 1800 & 0.0788 & 0.0505 \\
\hline 2970 & 0.0853 & 0.0405 \\
\hline 4680 & 0.0878 & 0.035 \\
\hline
\end{tabular}


Table S18. Raw data for the determination of order in $\mathrm{Et}_{3} \mathrm{~N}$ under conditions of general procedure $\mathrm{D}$ at 3 mol\% catalyst. The reaction was run with $1,1.5,2,3$, and 4 equiv of $\mathrm{Et}_{3} \mathrm{~N}$.<smiles>O=C(Nc1ccc(F)cc1)O[PH3+]</smiles>

1 equiv.

\begin{tabular}{|c|c|}
\hline time $(\min )$ & [3wa] \\
\hline 0 & 0 \\
\hline 48 & 0.001 \\
\hline 123 & 0.007 \\
\hline 430 & 0.025 \\
\hline 828 & 0.043 \\
\hline 1458 & 0.06 \\
\hline 1963 & 0.067 \\
\hline
\end{tabular}<smiles>[Te][Te][Te]</smiles>

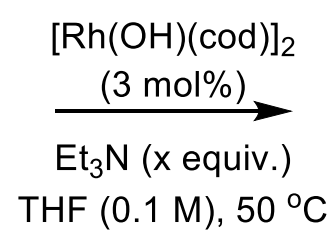

3 equiv.

\begin{tabular}{|c|c|}
\hline time(min) & [3wa] \\
\hline 0 & 0 \\
\hline 123 & 0.0102 \\
\hline 492 & 0.0543 \\
\hline 727 & 0.0637 \\
\hline 1288 & 0.0692 \\
\hline 1816 & 0.0713 \\
\hline
\end{tabular}

1.5 equiv.

$\begin{array}{ccc}\underline{\text { time }(\mathrm{min})} & & \text { [3wa] } \\ 0 & & 0 \\ 163 & 0.0247 \\ 384 & 0.0458 \\ 640 & 0.0607 \\ 1288 & 0.074 \\ 1778 & & 0.0762\end{array}$

2 equiv.

\begin{tabular}{|c|c|}
\hline time (min) & [3wa \\
\hline 0 & 0 \\
\hline 123 & 0.0107 \\
\hline 492 & 0.0555 \\
\hline 727 & 0.0657 \\
\hline 1288 & 0.0722 \\
\hline 1816 & 0.0745 \\
\hline 2986 & 0.075 \\
\hline
\end{tabular}

4 equiv.

\begin{tabular}{|c|c|}
\hline time(min) & [3wa] \\
\hline 0 & 0 \\
\hline 163 & 0.0533 \\
\hline 384 & 0.0702 \\
\hline 640 & 0.075 \\
\hline
\end{tabular}




\section{2-Evidence for Protodemetallation}

When we began to probe the functional group tolerance of different organoboron reagents on the transformation (Table $\mathbf{2 b}$ ), it was quickly realized that a variety of boronic acids were poor coupling partners with no clear electronic or steric trend. This led us to question the role of water in the reaction. Commercial samples of boronic acids exist as mixtures of oligomers, boroxine, and boronic acid. NMR analysis revealed that $2 \mathrm{a}$ exists predominately in the boroxine ( $>50 \%$ weight $\%$ ) form while most other boronic acids tested were predominately in the hydrated form. We hypothesized that oligomerization of boronic acids in situ (which is promoted by basic amines and low polarity solvents ${ }^{31}$ ) would result in the realease of water promoting a rapid protodemetallation pathway outcompeting the desired coupling. To test this hypothesis boronic acid $\mathbf{2} \mathbf{g}$ was recrystallized in water and subsequently submitted to the reaction conditions described in general procedure $C$. No boronic acid was detected by TLC 1 hour into the reaction. The boronic acid $\mathbf{2 g}$ was then recrystallized in $\mathrm{D}_{2} \mathrm{O}$ and submitted to the reaction conditions in general procedure $\mathrm{C}(0.45 \mathrm{mmol}$ of $\mathbf{2 g})$. The reaction was concentrated under reduced pressure at the 2 hour mark. The crude mixture was resuspended in EtOAc $(40 \mathrm{~mL})$ and extracted with $1 \mathrm{M} \mathrm{NaOH}(1 \times 30 \mathrm{~mL})$, saturated $\mathrm{NaHCO}_{3}(1 \times 30 \mathrm{~mL})$, and brine $(1 \times 30 \mathrm{~mL})$. The product was purified by column chromatography $\left(40 \% \mathrm{CH}_{2} \mathrm{Cl}_{2}\right.$ /hexanes). Product volatility made it difficult to obtain a yield thus an internal standard was added (1,3,5-trimethoxybenzene, $14.1 \mathrm{mg}$ ) resulting in a $19 \%$ isolated yield. The low yield can likely be attributed to product lost due to volatility. The ratio of deuterium incorporation was found to be $37 \%$. These results provide evidence for a kinetically fast protodemetallation which outcompetes isocyanate insertion. This side reaction seems to be exacerbated by the use of the blocked isocyanates given the stark contrast with prior work by Murakami on free isocyanates which suffered no such side reaction using boronic acids. ${ }^{18}$

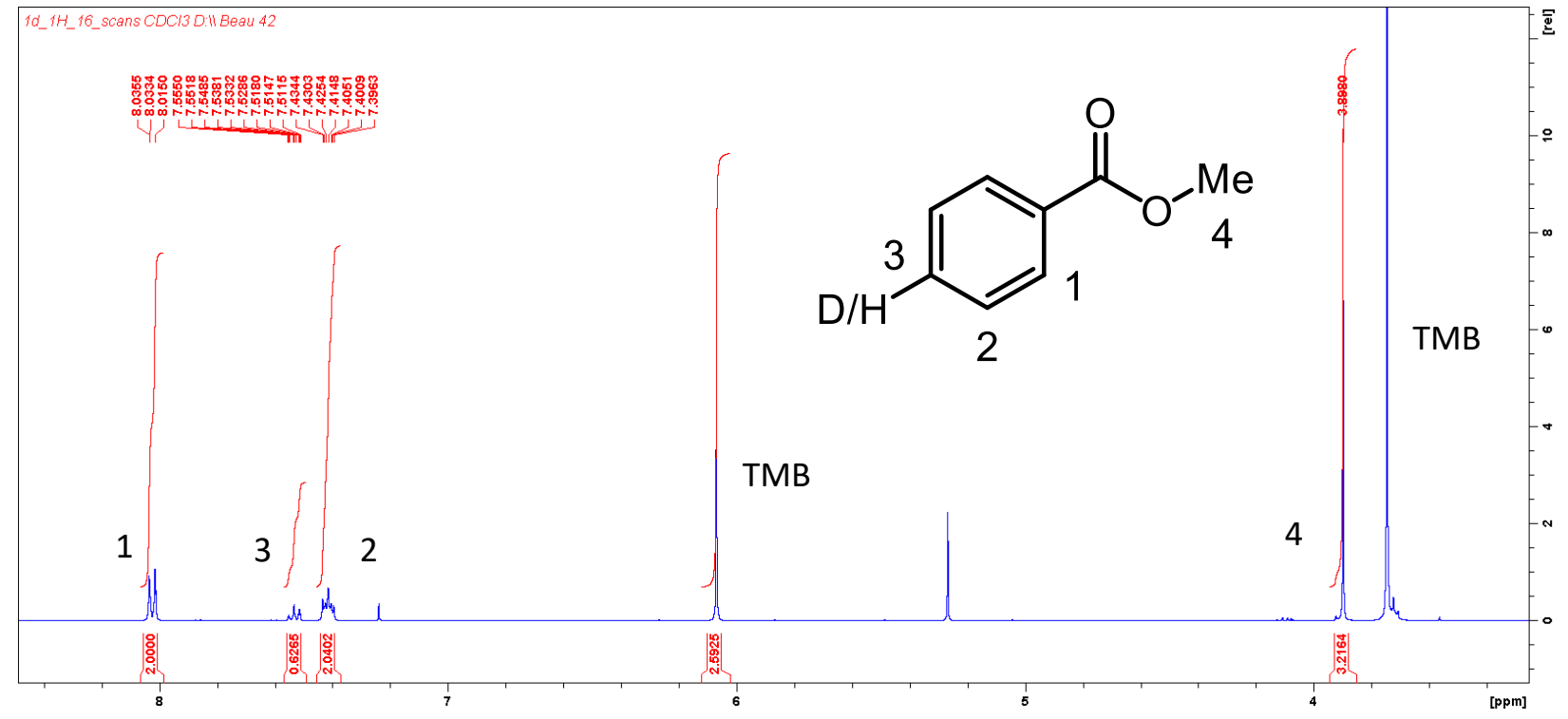




\section{3-Probing the existence of the Isocyanate}

The methylated derivative of blocked isocyanate 1a was prepared to probe the existence of the isocyanate intermediate due to its inability to act as a blocked isocyanate precursor. The carbamate was prepared using a literature procedure. ${ }^{2}$<smiles>CN(C(=O)Oc1ccccc1)c1ccccc1</smiles>

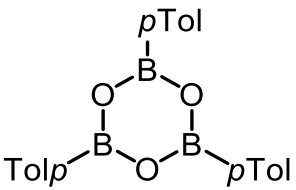

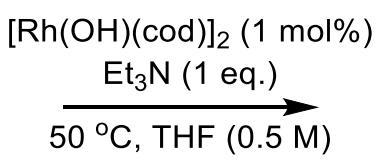<smiles>Cc1ccc(C(=O)N(C)c2ccccc2)cc1</smiles>

No product Detected

Under standard conditions, no product formation was detected. This result coupled with the absence of the involvement of the Rh catalyst in the rate determining step under some conditions is highly indicative of an isocyanate as a key reaction intermediate. ${ }^{28}$

\section{4-Probing reversibility - formation of tryptophan derived dimer (3vva)}

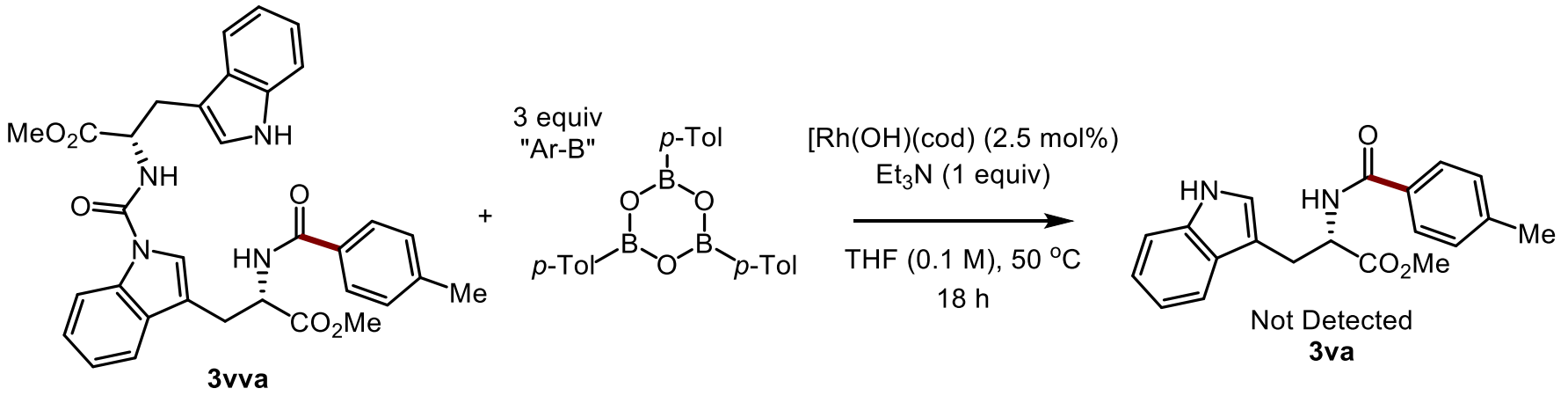

The dimer 3vva $(0.0288 \mathrm{~g}, 0.0496 \mathrm{mmol})$ was submitted to the reaction conditions described in general procedure $\mathbf{D}$ using boroxine $2 \mathrm{a}\left(0.0175 \mathrm{~g}, 0.0496 \mathrm{mmol}, 3\right.$ equiv “Ar-B"), $\mathrm{Et}_{3} \mathrm{~N}\left(6.9 \times 10^{-3} \mathrm{~mL}, 0.050 \mathrm{mmol}\right)$, and $[\mathrm{Rh}(\mathrm{OH})(\mathrm{cod})]_{2}\left(0.0056 \mathrm{~g}, 1.2 \times 10^{-3} \mathrm{mmol}\right)$ at $80^{\circ} \mathrm{C}$. The reaction was dried down after 18 hours with no sign of product formation or disappearance of starting material by TLC. The crude reaction mixture was analyzed by NMR and revealed no detectable product formed with $66 \%$ starting material remaining. These results suggest the formation of the dimer under the reaction conditions is not reversible.

\footnotetext{
1 Jung, H. H.; Buesking, A. W.; Ellman, J. A. Highly Functional Group Compatible Rh-Catalyzed Addition of Arylboroxines to Activated $\mathrm{N}$-tert-Butanesulfinyl Ketimines. Org. Lett. 2011, 13, 3912-3915.

2 Jacquemard, U.; Beneteau, V.; Lefoix, M.; Routier, S.; Merour, J.-Y.; Coudert, G. Mild and Selective Deprotection of Carbamates with $\mathrm{Bu}_{4} \mathrm{NF}$. Tetrahedron 2004, 60, 10039-10047.

${ }^{3}$ Martinez, R.; Ramon, D. J.; Yus, M. Unmodified Nano-Powder Magnetite Catalyzes a Four-Component Aza-Sakurai Reaction. Adv. Synth. Catal. 2008, 350, 1235-1240.
} 
${ }^{4}$ Gauthier, D. R. Jr.; Sherry, B. D.; Cao, Y.; Journet, M.; Humphrey, G.; Itoh, T.; Mangion, I.; Tschaen, D. M. Highly Efficient Synthesis of HIV NNRTI Doravirine. Org. Lett. 2015, 17, 1353-1356.

${ }^{5}$ Treventis Corporation. Carbamate Compounds and Methods of use in Diseases of the Nervous System. WO2014039526 A2, March 13 ${ }^{\text {th }}, 2014$.

${ }^{6}$ Ghodsinia, S. S. E.; Akhlaghinia, B. A High-Yielding, Expeditious, and Multicomponent Synthesis of Urea and Carbamate Derivatives by Using Triphenylphosphine/Trichloroisocyanuric Acid System. Phosphorus Sulfur 2015, 191, 104.

7 Gutschow, M. One-Pot Reactions of $N$-(Mesyloxy)phthalimides with Secondary Amines to 2-Ureidobenzamides, 2Ureidobenzoic Acids, Ethyl 2-Ureidobenzoates, or Isatoic Anhydrides. J. Org. Chem. 1999, 64, 5109-5115.

8 Doyle, M. P.; Pieters, R. J.; Taunton, J.; Pho, H. Q. Synthesis of Nitrogen-Containing Polycycles via Rhodium(II)Induced Cyclization-Cycloaddition and Insertion Reactions of $\mathrm{N}$-(Diazoacetoacetyl)amides. Conformational Control of Reaction Selectivity. J. Org. Chem. 1991, 56, 820-829.

${ }^{9}$ Synthesis: Figlus, M.; Tarruella, A. C.; Messer, A.; Sollis, S. L.; Hartley, R. C. Low Molecular Weight MPEG-Assisted Organic Synthesis. Chem. Commun. 2010, 46, 4405-4407. Characterization: Gately, D. A.; Norton, J. R.; Goodson, P. A. Insertion of Isocyanates, $\mathrm{CO}_{2}$, and Ethylene Carbonate into the $\mathrm{Zr}-\mathrm{C}$ and $\mathrm{Zr}-\mathrm{N}$ Bonds of Imine Complexes. Construction of Chiral Centers Like Those in $\alpha$-Amino Acids. J. Am. Chem. Soc. 1995, 117, 986-996.

${ }^{10}$ Busschaert, N.; Kirby, I. L.; Young, S.; Coles, S. J.; Horton, P. N.; Light, M. E.; Gale, P. A. Squaramides as Potent Transmembrane Anion Transporters. Angew. Chem. Int. Ed. 2012, 51, 4426-4430.

${ }_{11}$ Yamauchi, D.; Nishimura, T.; Yorimitsu, H. Hydroxoiridium-Catalyzed Hydroalkylation of Terminal Alkenes with Ureas by C(sp $\left.{ }^{3}\right)-H$ Bond Activation. Angew. Chem. Int. Ed. 2017, 56, 7200-7204.

${ }^{12}$ Organoboron compound $\mathbf{2 a}$ was used as received from the commercial supplier (Combi-blocks). It was determined that a 2.1:1 mixture of $\mathrm{RB}(\mathrm{OH})_{2}:[\mathrm{RBO}]_{3}$ was present. Equivalents based on the number of "Ar- $\mathrm{B}$ " presented in the communication to simplify comparisons. The mmol amount of $\mathbf{2 a}$ is reported with reference to the boroxine taking into account the presence of this mixture. It is noteworthy that fully dehydrating the parent mixture resulted in not change in yield with the optimized conditions reported in general procedure $C$.

${ }^{13}$ Sharma, R.; Vishwakarma, R. A.; Bharate, S. B. Ligand-Free Copper-Manganese Spinel Oxide-Catalyzed Tandem One-Pot C-H Amidation and N-Arylation of Benzylamines: A Facile Access to 2-Arylquinazolin-4(3H)-ones. Adv. Synth. Catal. 2016, 358, 3027-3033.

${ }^{14}$ Alapati, M. L. P. R.; Abburu, S. R.; Mutyala, K. R.; Mukkamala, S. B. Copper(I) lodide-Catalyzed Amidation of Phenylboronic Acids/Aryl Bromides Using 4-Dimethylaminopyridine as Ligand. Synth. Comm. 2016, 46, 1242-1248.

${ }^{15}$ Wu, Y.; Wang, S.; Zhang, L.; Yang, G.; Zhu, X.; Zhou, Z.; Zhu, H.; Wu, S. Cyclopentadienyl-Free Rare-Earth Metal Amides $\left.\left[\left\{\left(\mathrm{CH}_{2} \mathrm{SiMe}\right)_{2}\right)\left\{\left(2,6-i \mathrm{Pr}_{2} \mathrm{C}_{6} \mathrm{H}_{3}\right) \mathrm{N}\right\}_{2}\right\} \mathrm{Ln}\left\{\mathrm{N}\left(\mathrm{SiMe}_{3}\right)_{2}\right\}(\mathrm{THF})\right]$ as Highly Efficient Versatile Catalysts for C-C and C-N Bond Formation. Eur. J. Org. Chem. 2010, 326-332.

${ }^{16}$ Howard, E. L.; Guzzardi, Tsanova, V. G.; Stika, A.; Patel, B. Highly Efficient Copper-Catalyzed Amidation of Benzylic Hydrocarbons Under Neutral Conditions. Eur. J. Org. Chem. 2018, 794-797.

17 Jo, Y.; Ju, J.; Choe, J.; Song, K. W.; Lee, S. The Scope and Limitation of Nickel-Catalyzed Aminocarbonylation of Aryl Bromides from Formamide Derivatives. J. Org. Chem. 2009, 74, 6358-6361.

18 Rao, Y.; Li, X.; Danishefsky, S. J. Thio FCMA Intermediates as Strong Acyl Donors: A General Solution to the Formation of Complex Amide Bonds. J. Am. Chem. Soc. 2009, 131, 12924-12926.

${ }^{19}$ Miura, T.; Takahashi, Y.; Murakami, M. Rhodium-Catalyzed Addition Reaction of Aryl- and Alkenylboronic Acids to Isocyanates. Chem. Commun. 3577-3579.

${ }^{20}$ Nozawa-Kumada, K.; Kadokawa, J.; Kameyama, T.; Kondo, Y. Copper-Catalyzed sp ${ }^{3}$ C-H Aminative Cyclization of 2Alkyl- $\mathrm{N}$-arylbenzamides: An Approach for the Synthesis of $\mathrm{N}$-Aryl-isoindolinones. Org. Lett. 2015, 17, 4479-4481.

${ }^{21}$ Kumar, K. N.; Sreeramamurthy, K.; Palle, S.; Mukkanti, K.; Das, P. Dithiocarbamate and DBU-Promoted Amide Bond Formation Under Microwave Conditions. Tetrahedron Lett. 2010, 51, 899-902.

${ }^{22}$ Zhang, D.-X.; Xiang, S.-K.; Hu, H.; Tan, W.; Feng, C.; Wang, B.-Q.; Zhao, K.-Q.; Hu, P.; Yang, H. An Improved Protocol for Synthesis of $\mathrm{N}$-Arylamides and Benzoxazoles by the Copper-Catalyzed Reaction of Aryl Halides with Nitriles. Tetrahedron, 2013, 69, 10022-10029.

${ }^{23}$ Al-Nadaf, A.; Sheikha, G. A.; Taha, M. O. Elaborate Ligand-Based Pharmacophore Exploration and QSAR Analysis Guide the Synthesis of Novel Pyridinium-Based Potent $\beta$-Secretase Inhibitory Leads. Bioorg. Med. Chem. 2010, 18, 3088-3115. 
${ }^{24}$ Jiang, H.; Liu, B.; Li, Y.; Wang, A.; Huang, H. Synthesis of Amides via Palladium-Catalyzed Amidation of Aryl Halides. Org. Lett. 2011, 13, 1028.

25 a) Hutchby, M.; Houlden, C. E.; Ford, J. F.; Tyler, S. N. G.; Gagné, M. R.; Lloyd-Jones, G. C.; Booker-Millburn, K. I. Hindered Ureas as Masked Isocyanates: Facile Carbamoylation of Nucleophiles under Neutral Conditions. Angew. Chem. Int. Ed. 2009, 48, 8721-8724. b) Cai, K.; Ying, H.; Cheng, J. Dynamic Ureas with Fast and pH-Independent Hydrolytic Kinetics. Chem. Eur. J. 2018, 24, 7345-7348.

${ }^{26}$ a) Burés, J. A Simple Graphical Method to Determine the Order in Catalyst. Angew. Chem. Int. Ed. 2016, 55, 20282031. b) Burés, J. Variable Time Normalization Analysis: General Graphical Elucidation of Reaction Orders from Concentration Profiles. Angew. Chem. Int. Ed. 2016, 55, 16084-16087.

27 Duan, W.-L.; Iwamura, H.; Shintani, R.; Hayashi, T. Chiral Phosphine-Olefin Ligands in the Rhodium-Catalyzed Asymmetric 1,4-Addition Reaction. J. Am. Chem. Soc. 2007, 129, 2130-2138.

${ }^{28}$ a) Hie, L.; Nathel, N. F. F.;Hong, X.; Yang, Y.-F.; Houk, K. N.; Garg, N. K. Nickel-Catalyzed Activation of Acyl C-O Bonds of Methyl Esters. Angew. Chem. Int. Ed. 2016, 55, 2810-2814. b) Halima, T. B.; Vandavasi, J. K.; Shkoor, M.; Newman, S. G. A Cross-Coupling Approach to Amide Bond Formation from Esters. ACS Catal. 2017, 7, 2176-2180; c) Halima, T. B.; Masson-Makdissi, J.; Newman, S. G. Nickel-Catalyzed Amide Bond Formation from Methyl Esters. Angew. Chem. Int. Ed. 2018, 57, 12925-12929.

${ }^{29}$ Lim, D. S. W.; Lew, T. T. S.; Zhang, Y. Direct Amidations of N-Boc- and N-Cbz-Protected Amines via RhodiumCatalyzed Coupling of Arylboroxines and Carbamates. Org. Lett. 2015, 17, 6054-6057.

${ }^{30}$ Ghosh, A. K.; Brindisi, M. Organic Carbamates in Drug Design and Medicinal Chemistry. J. Med. Chem. 2015, 58, 2895-2940.

${ }^{31}$ (a) Korich, A. L.; lovine, P. M. Boroxine Chemistry and Applications: A Perspective. Dalt. Trans. 2010, 39, 14231431; (b) Tonkunaga, Y. Boroxine Chemistry: From Fundamental Studies to Applications in Supramolecular and Synthetic Organic Chemistry. Heterocycles 2013, 87, 991-1021. 

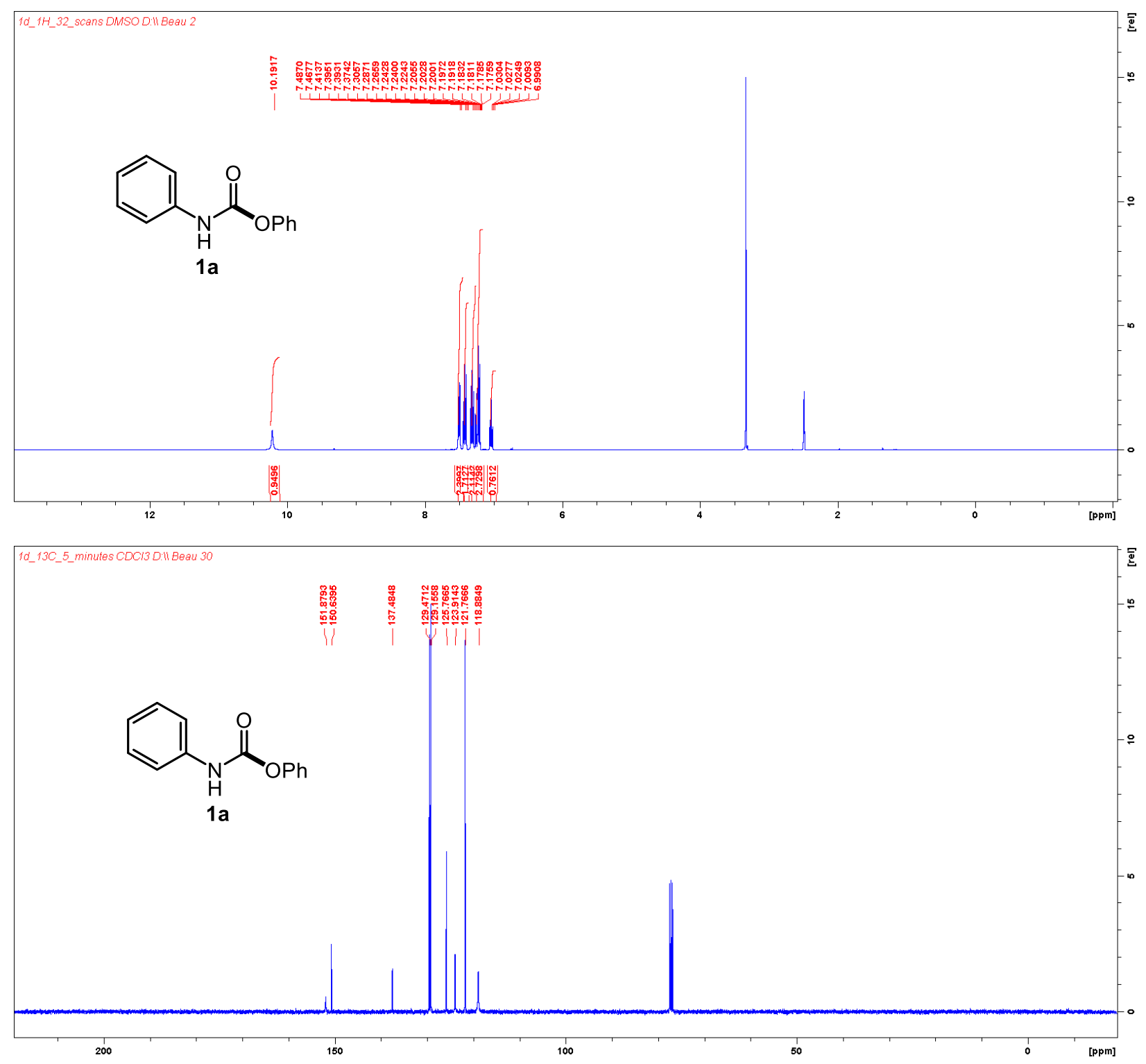

Proton: JD-9-11-1 Carbon: JD-13-1-SM 

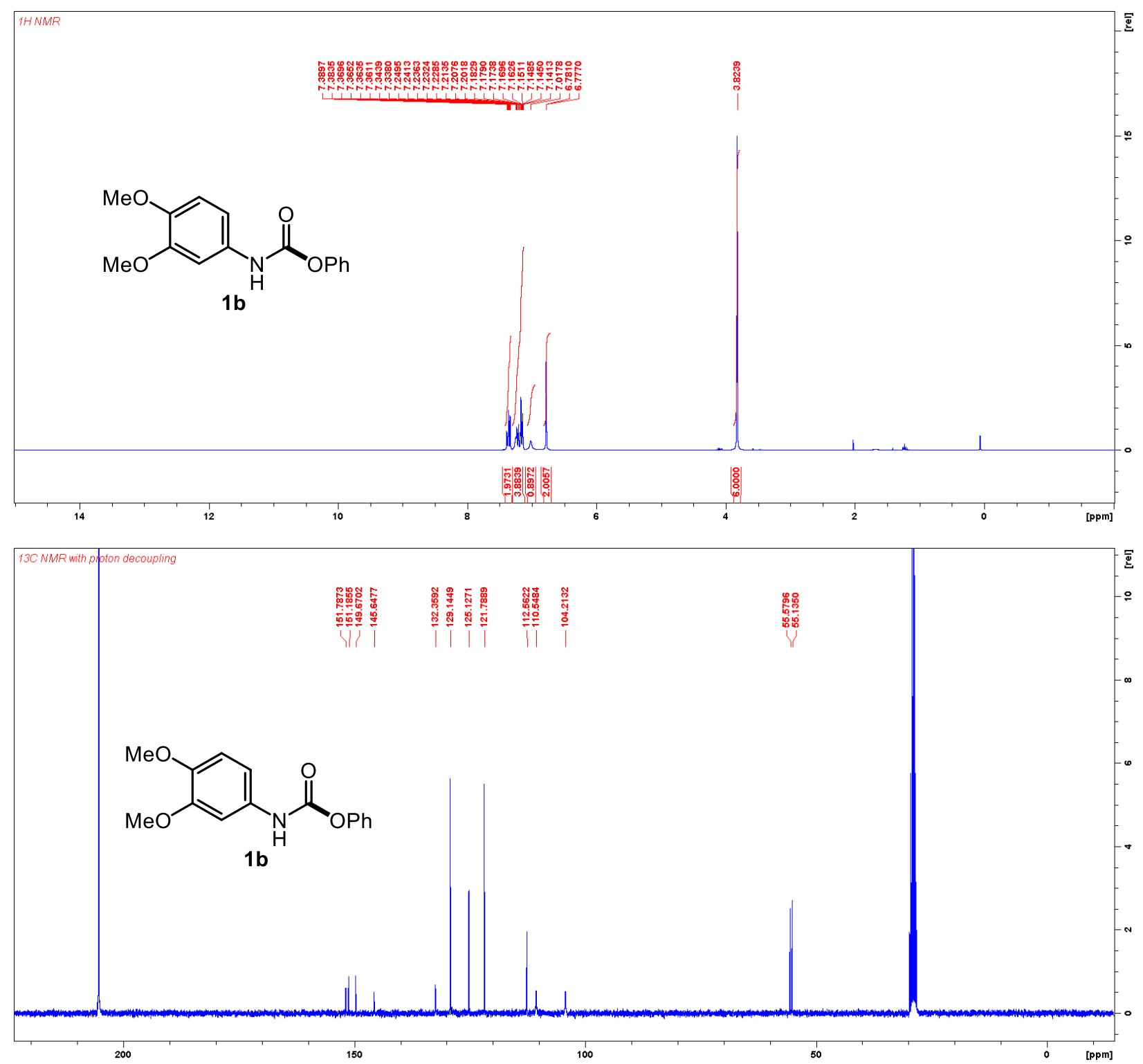

Proton: JD-8-64-1A 

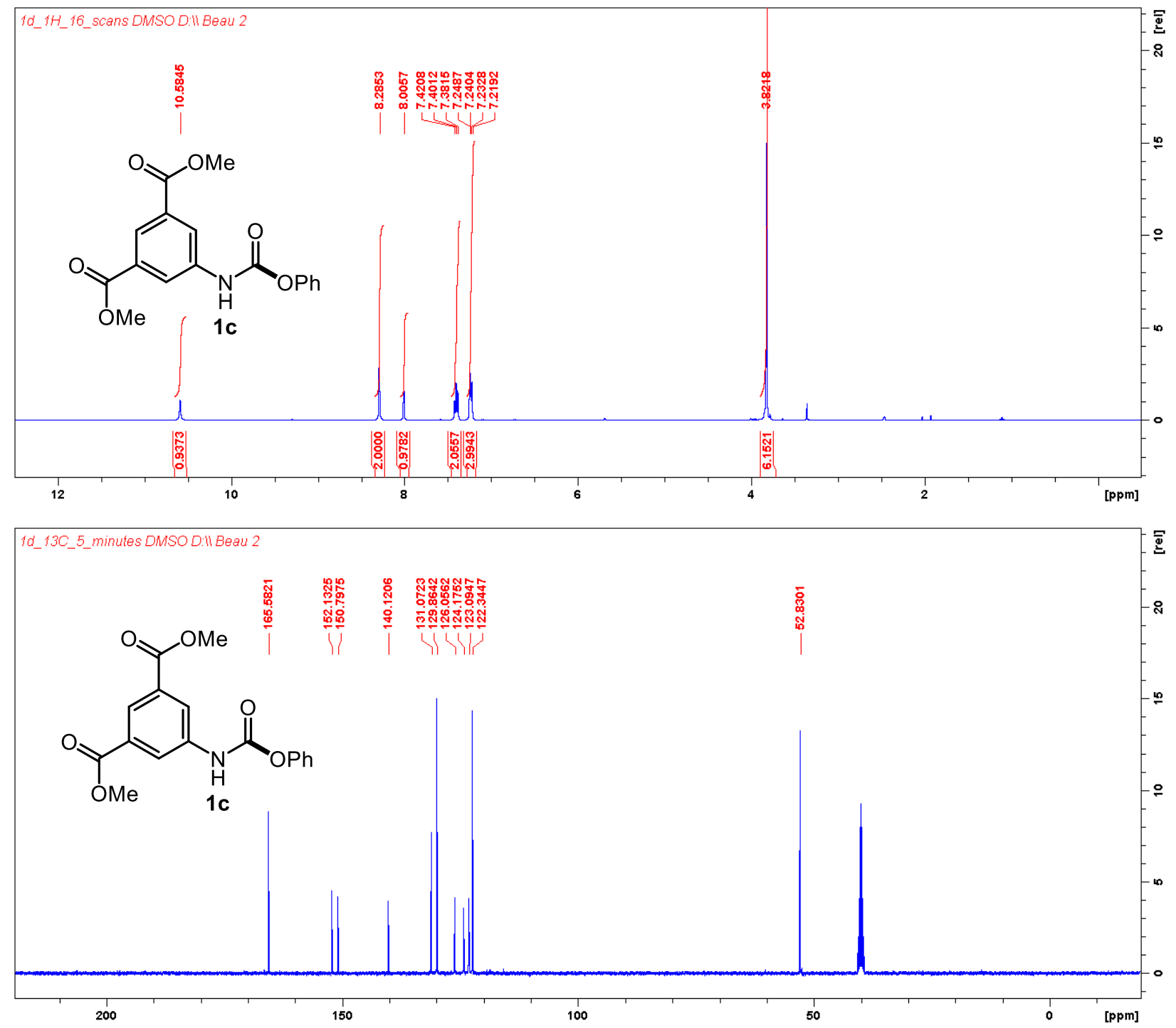

Proton: JD-13-1-2G Carbon: JD-13-1-2G 

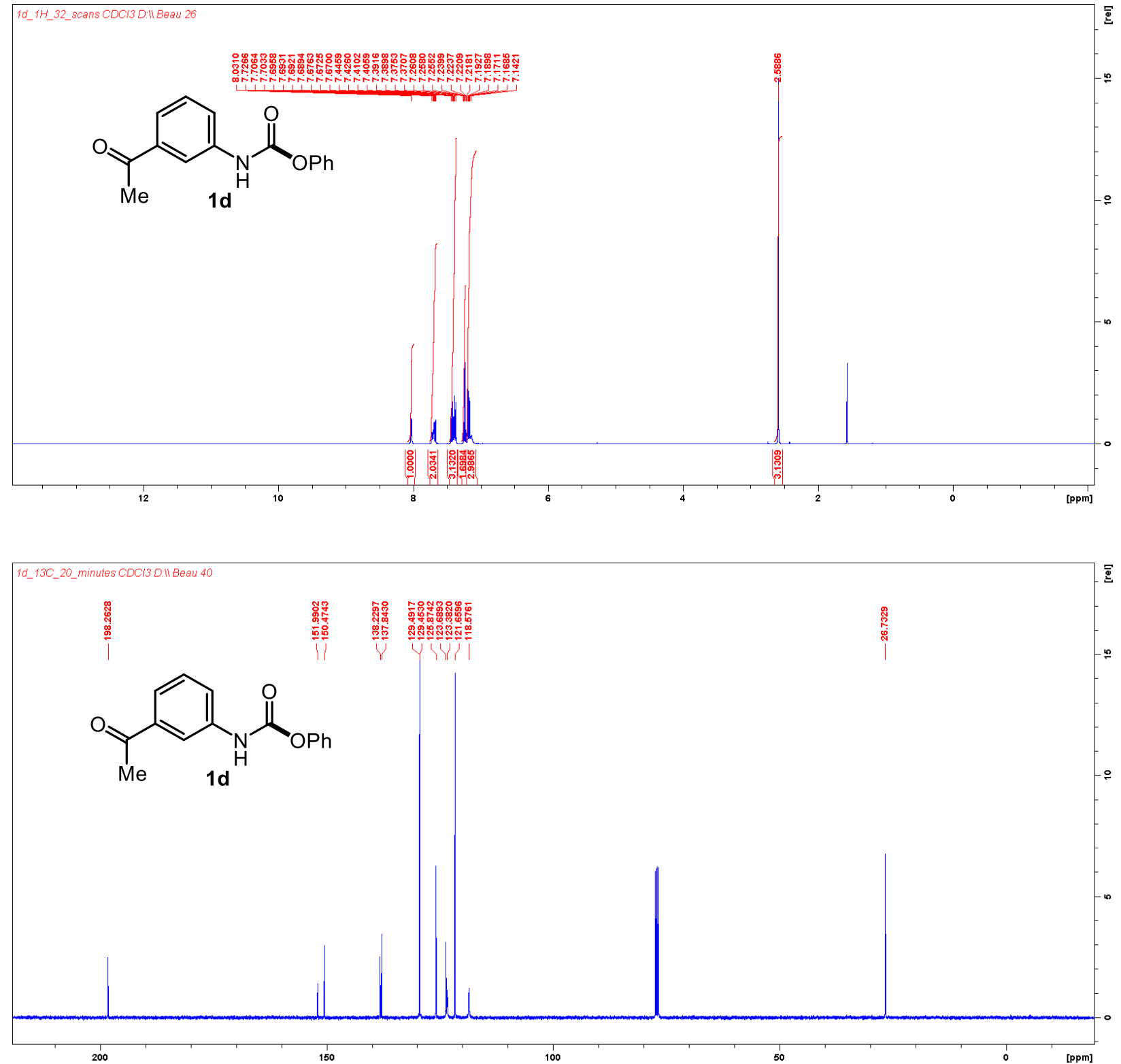

Proton: JD-10-17-6/JD-10-2-1 Carbon: JD-10-17-6 

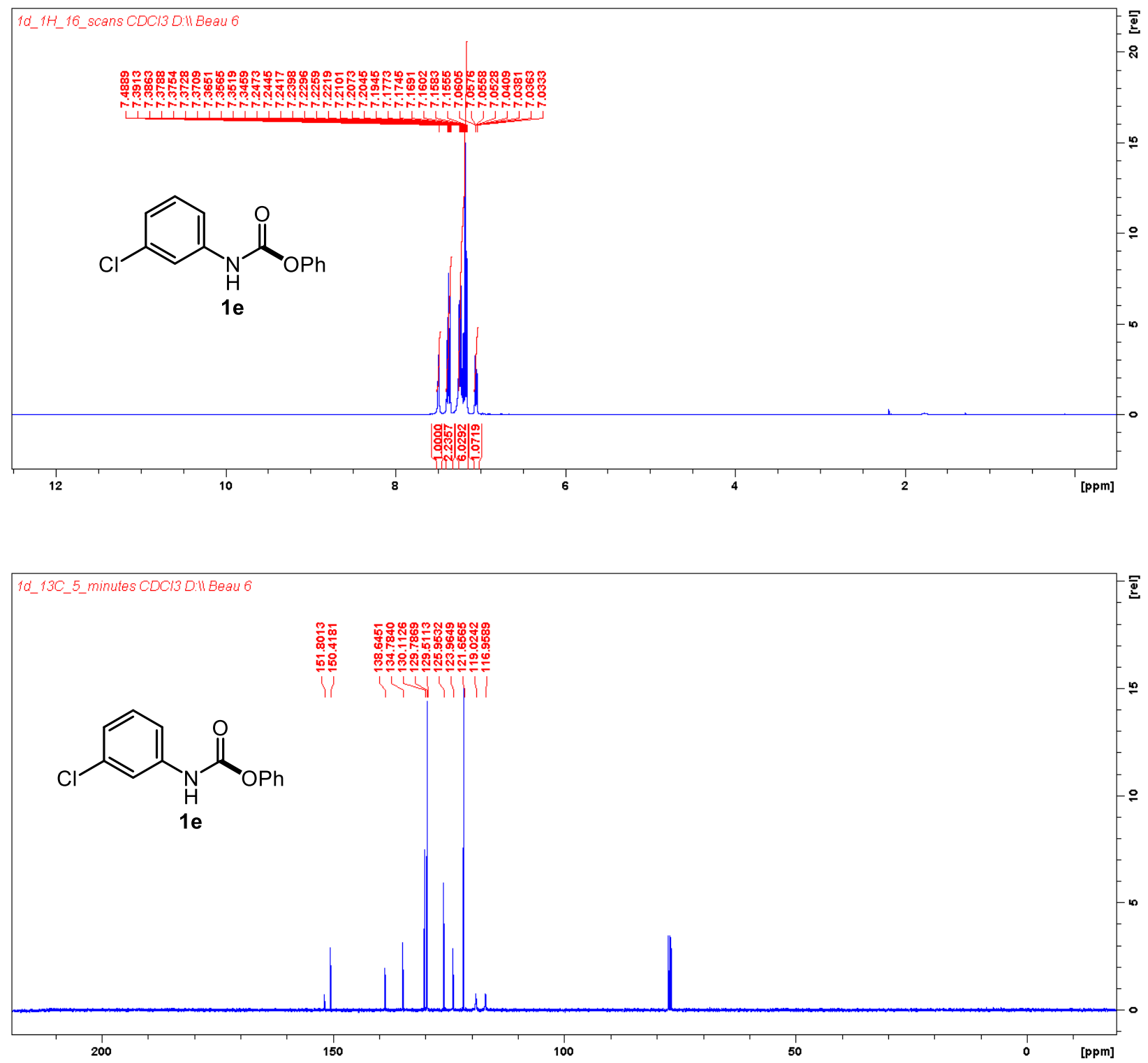

Proton: JD-13-1-1E Carbon: JD-13-1-1E 

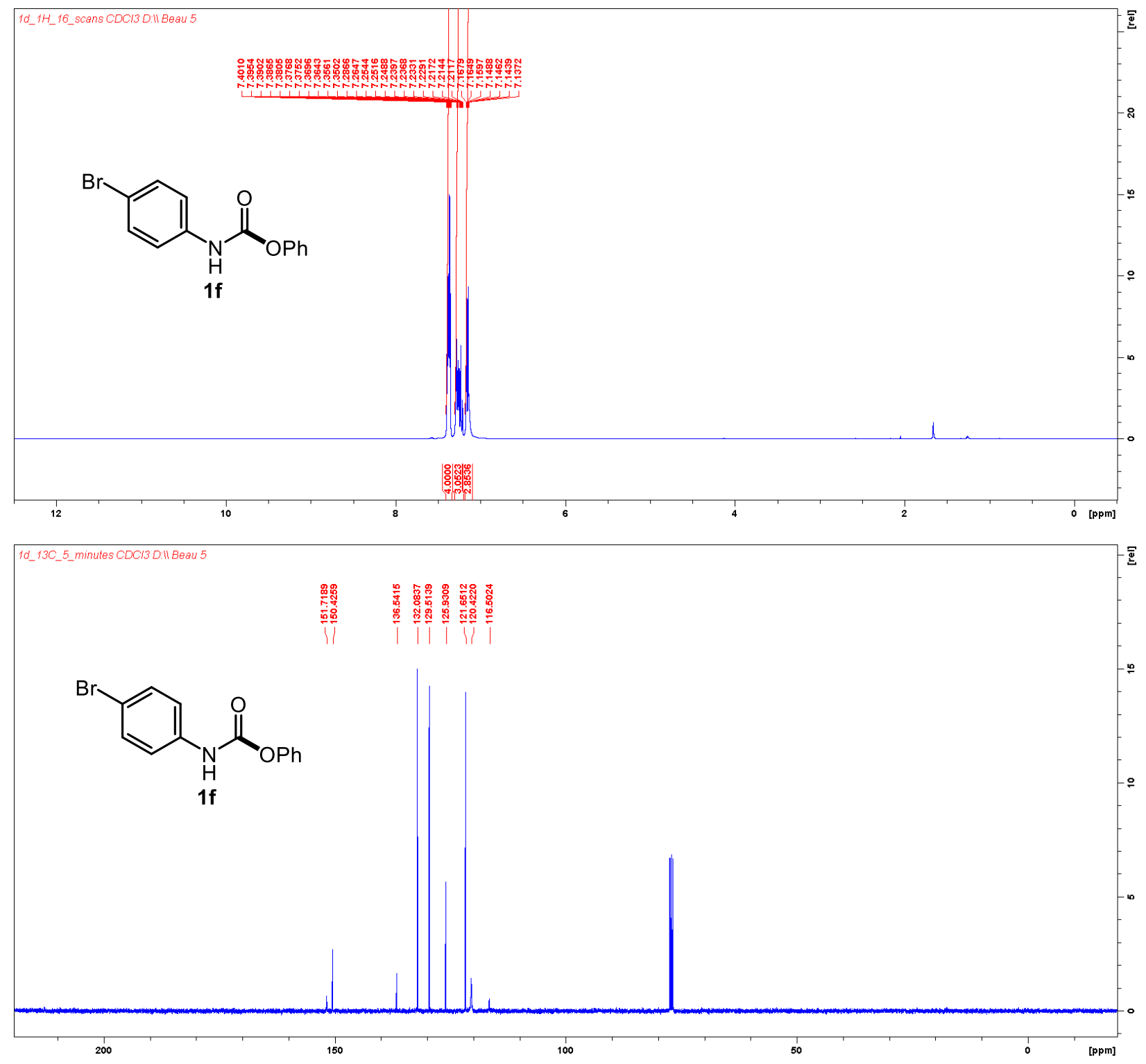

Proton: JD-13-1-1D Carbon: JD-13-1-1D 

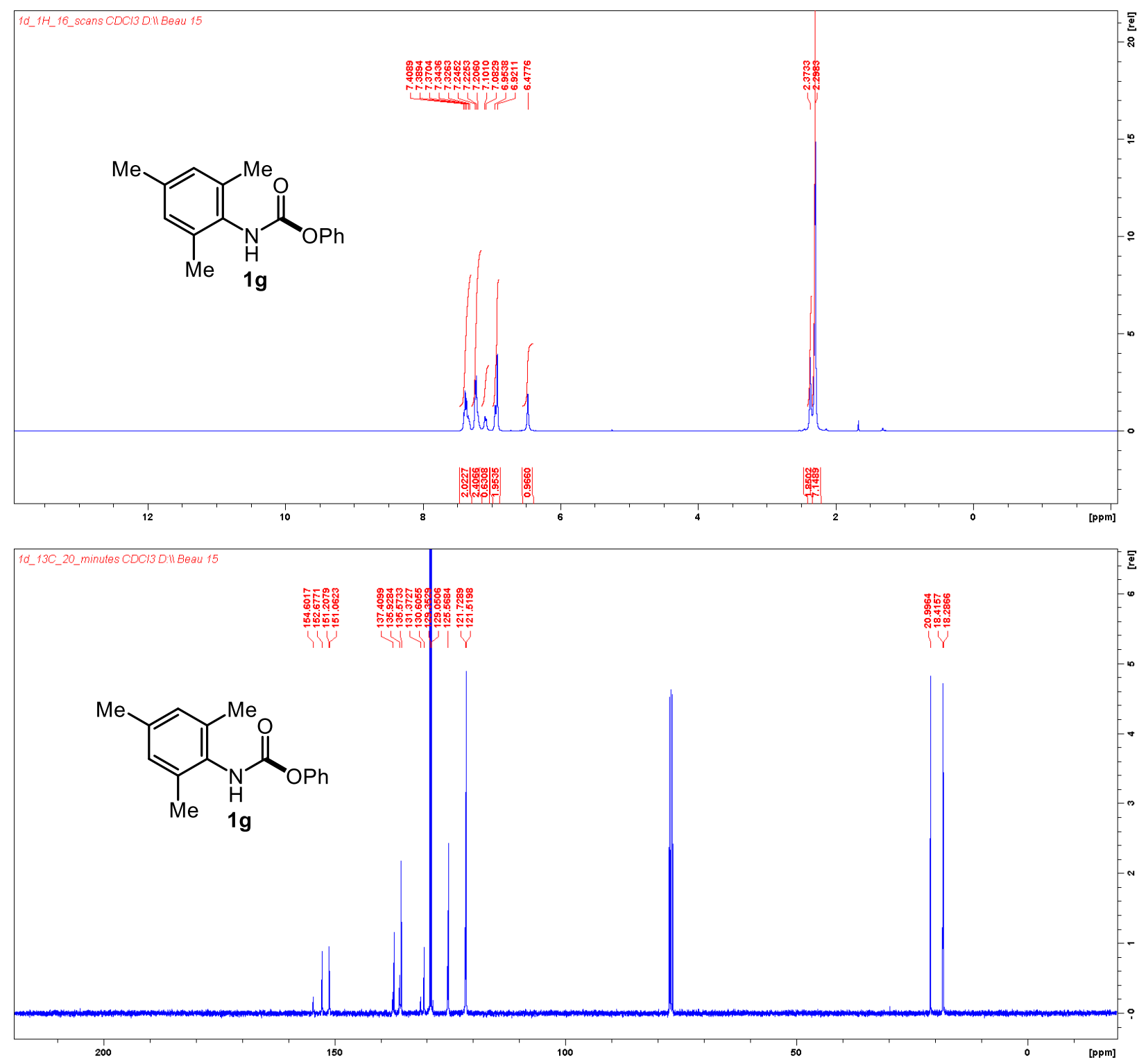

Proton: JD-10-17-6_2 Carbon: JD-10-17-7 

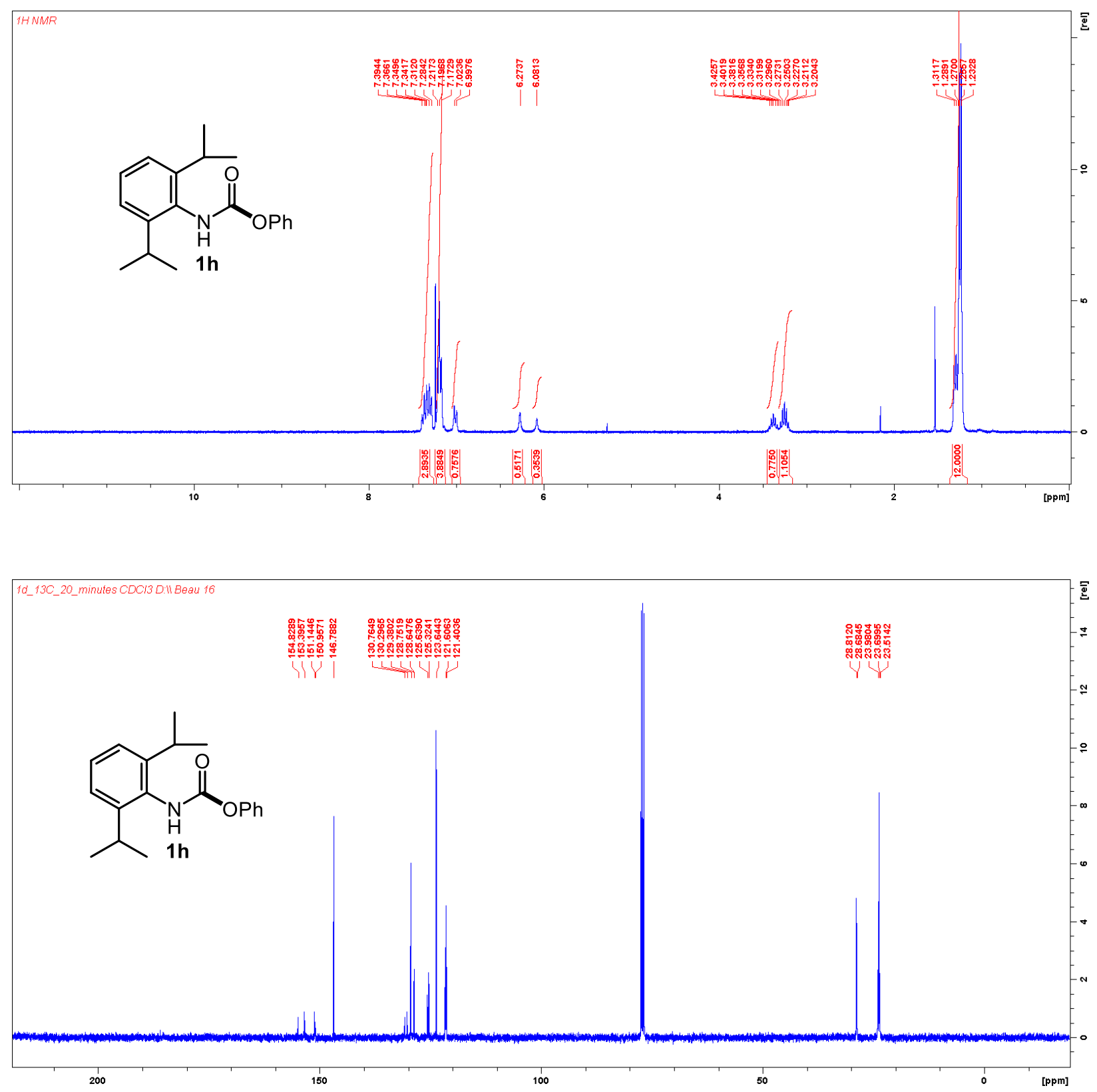

Proton: JD-10-23-6A Carbon: JD-10-23-4 

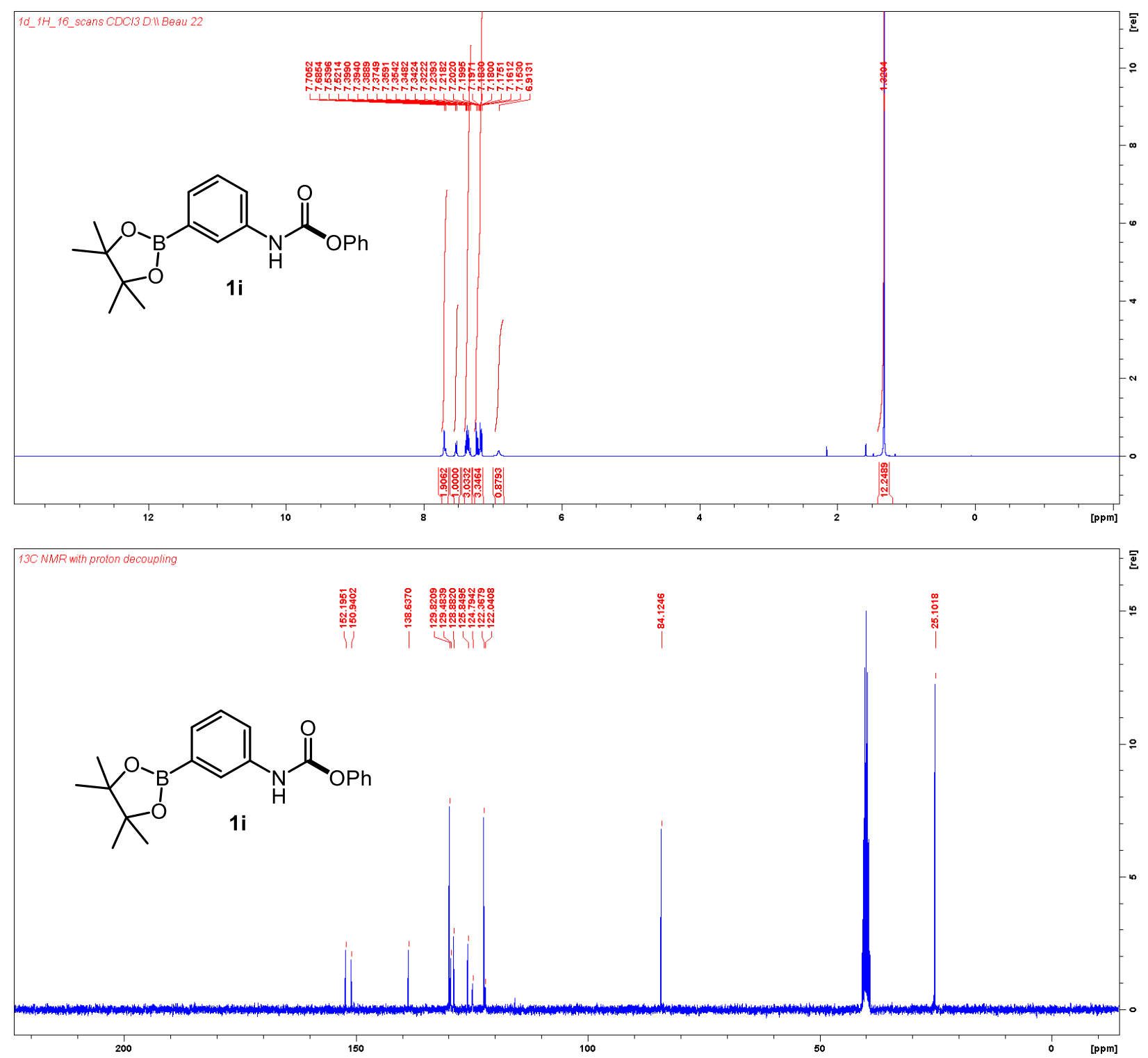

Proton: JD-10-92-3 Carbon: JD-13-2-2B 

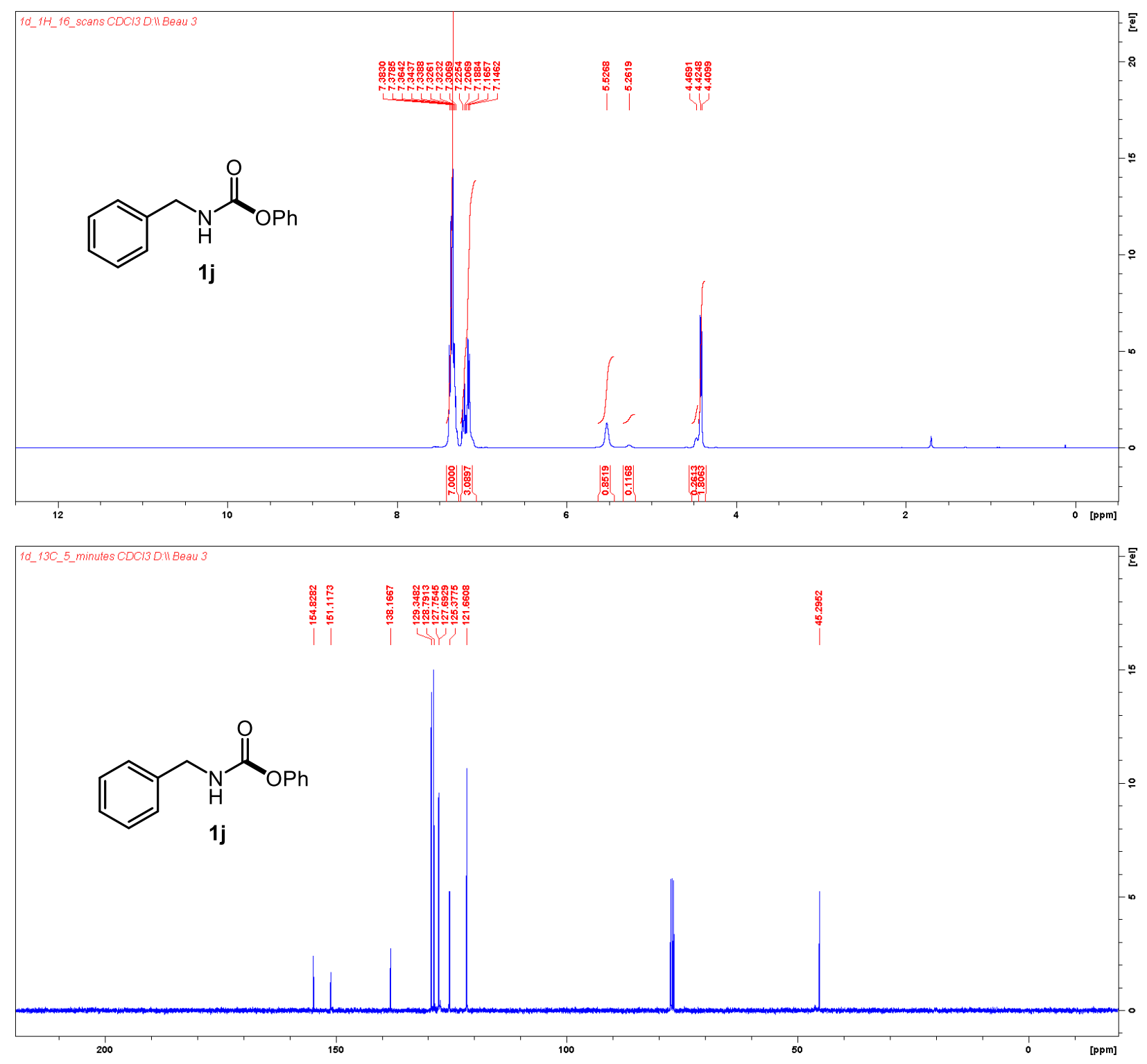

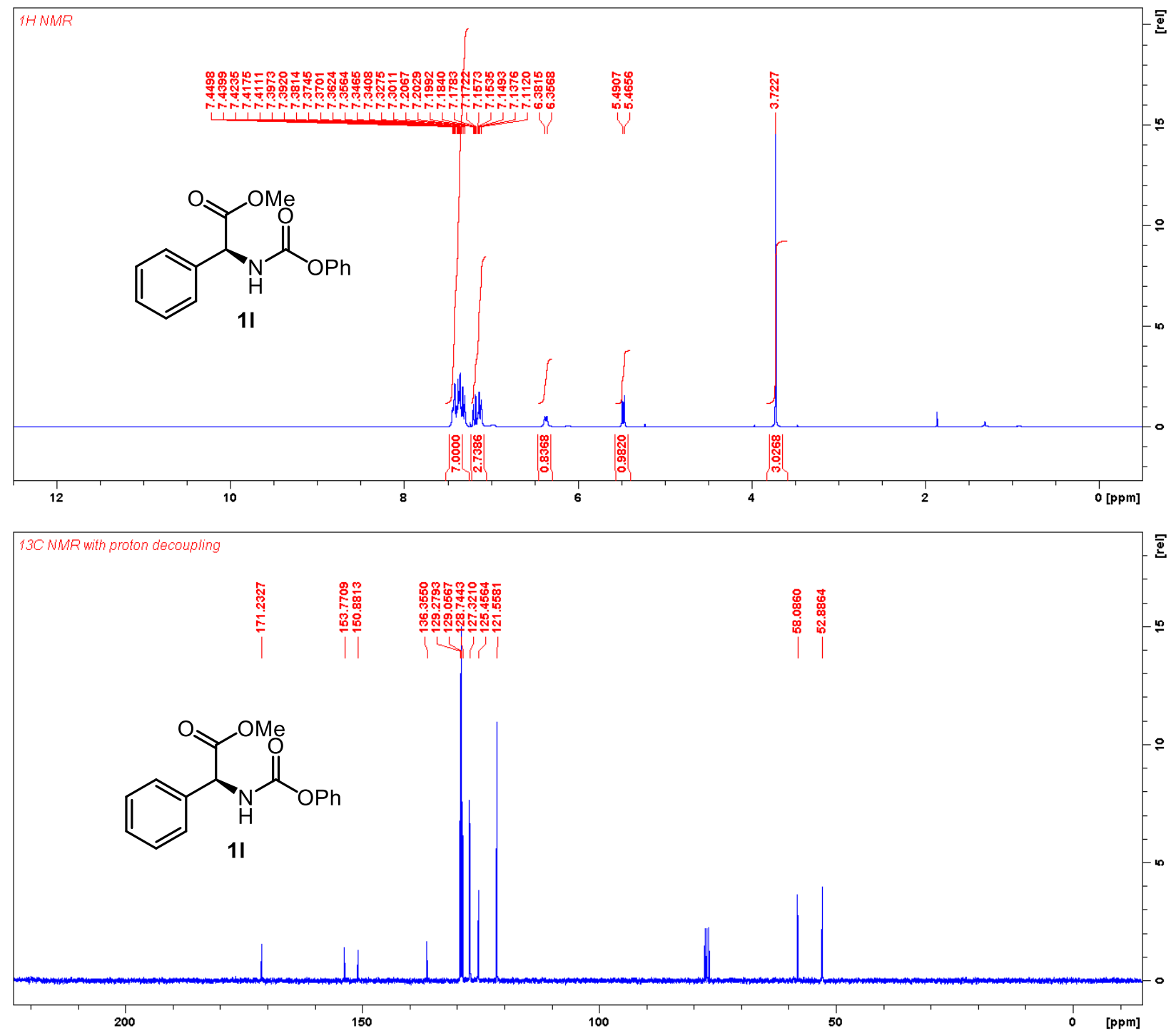

Proton: JD-13-6-2B Carbon: JD-13-6-2B 


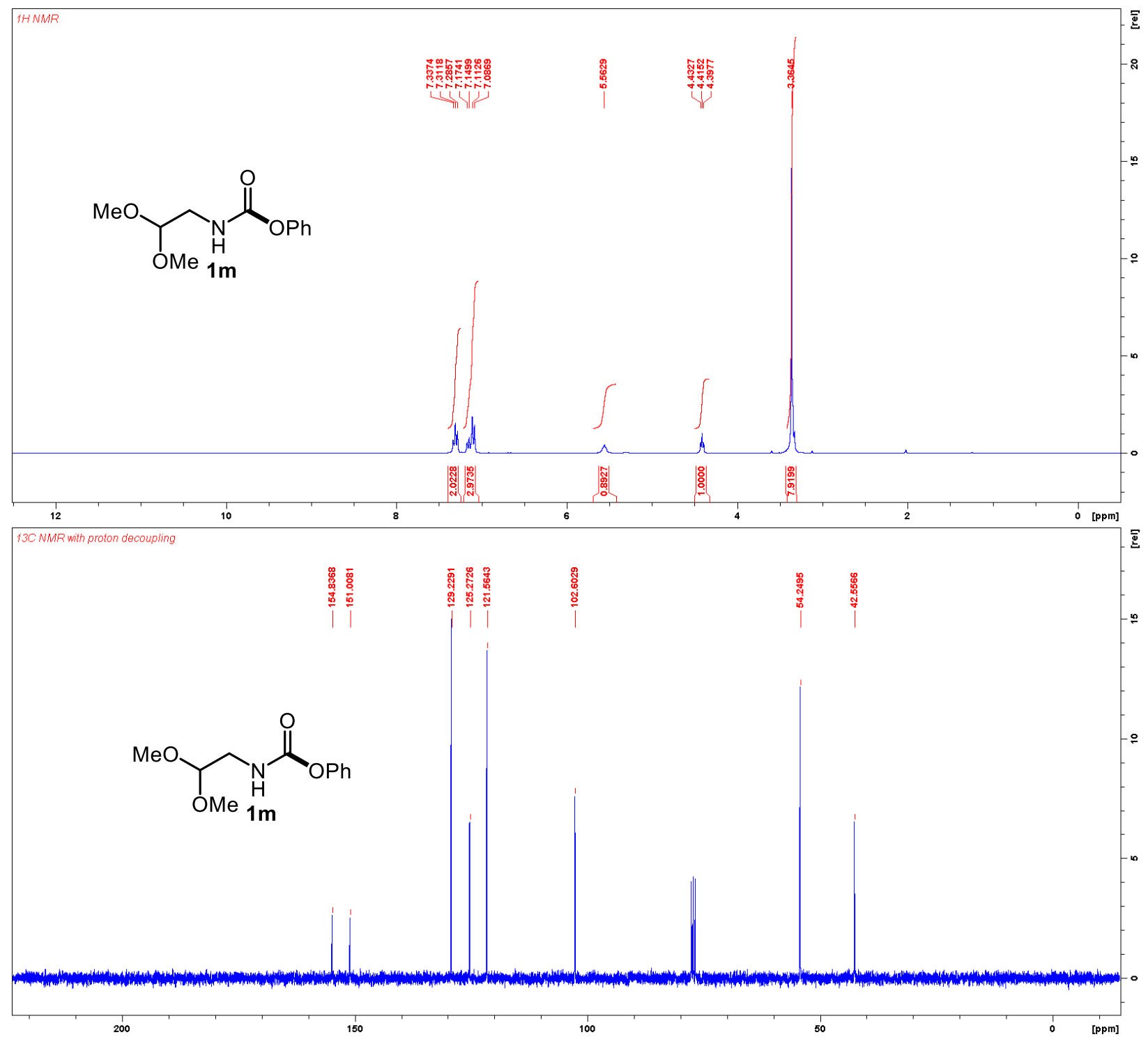

Proton: JD-10-81-4 Carbon: JD-10-81-4 

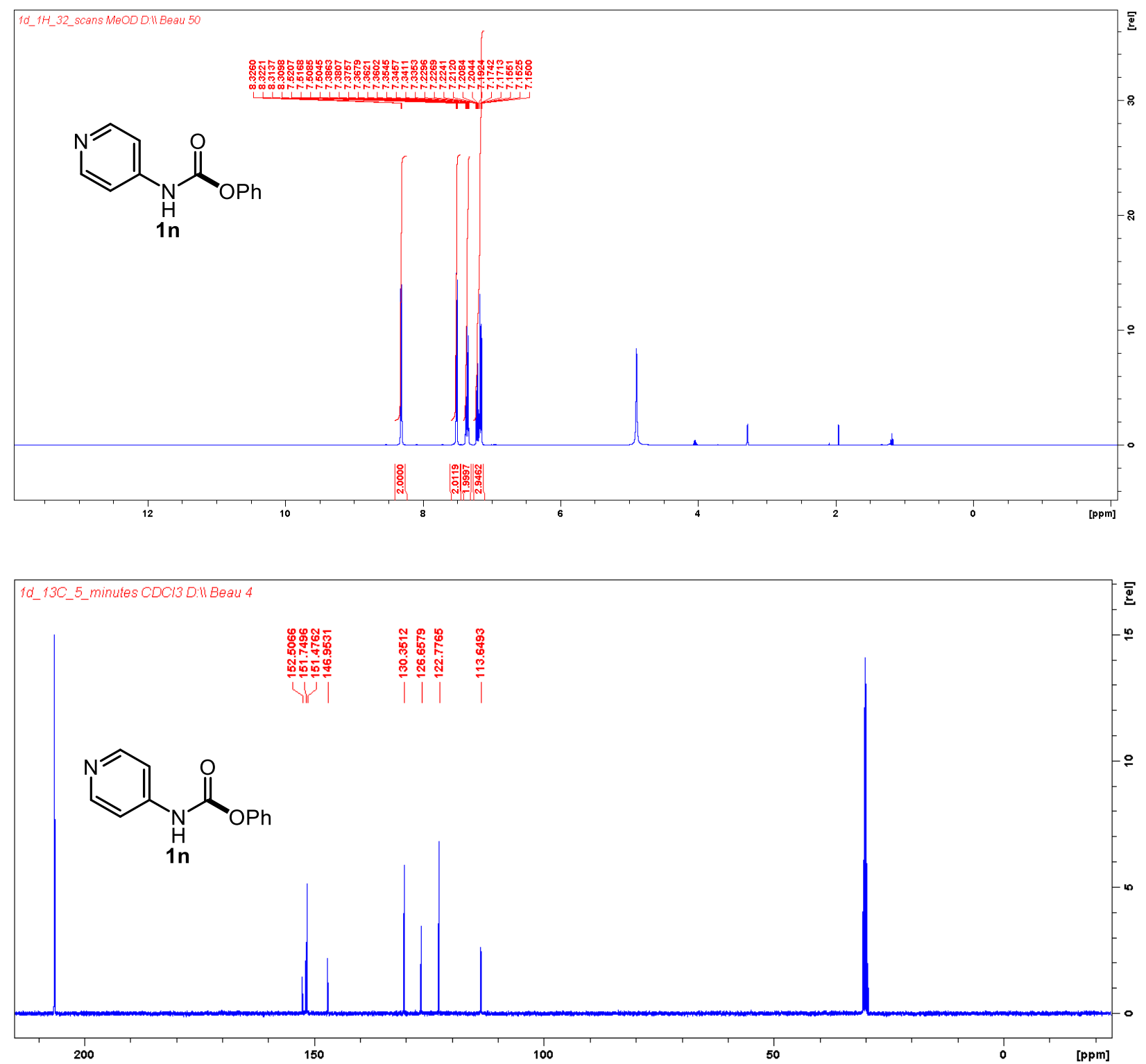

Proton: Unsure Carbon: JD-13-1-1I 

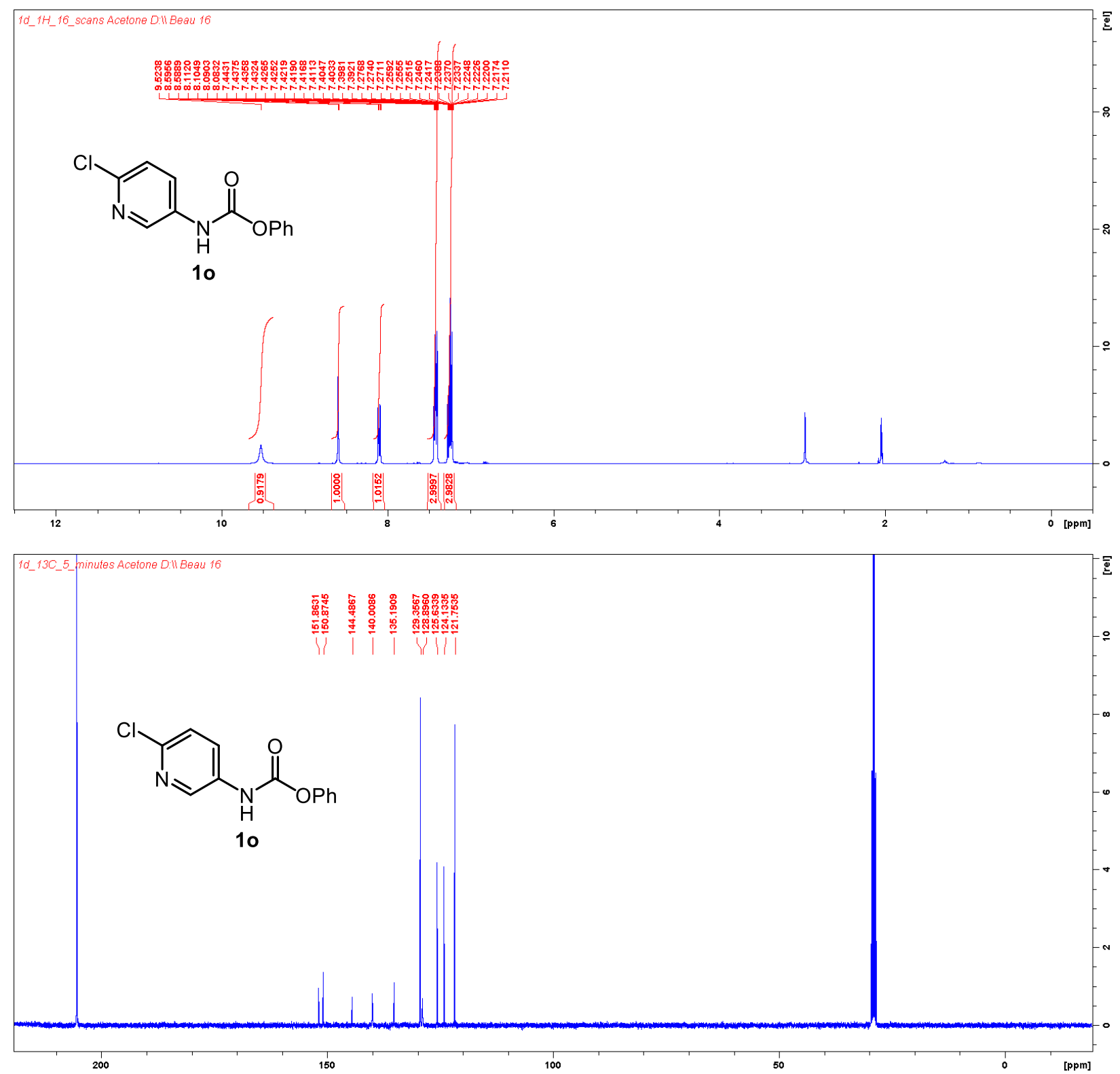

Proton: JD-13-3-3B Carbon: JD-13-3-3B 

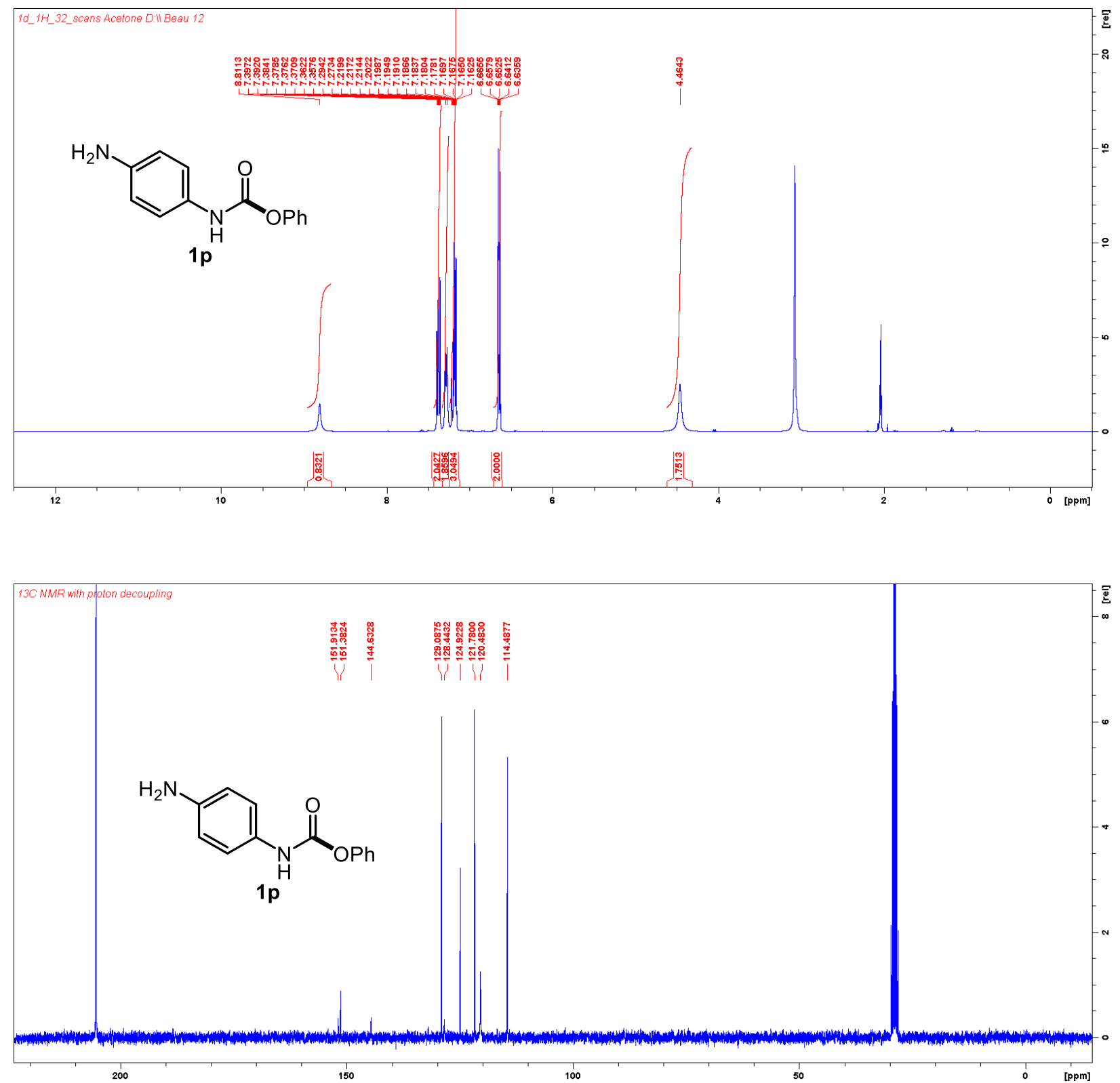

Proton: JD-12-62-2B Carbon: JD-12-62-2BBB 

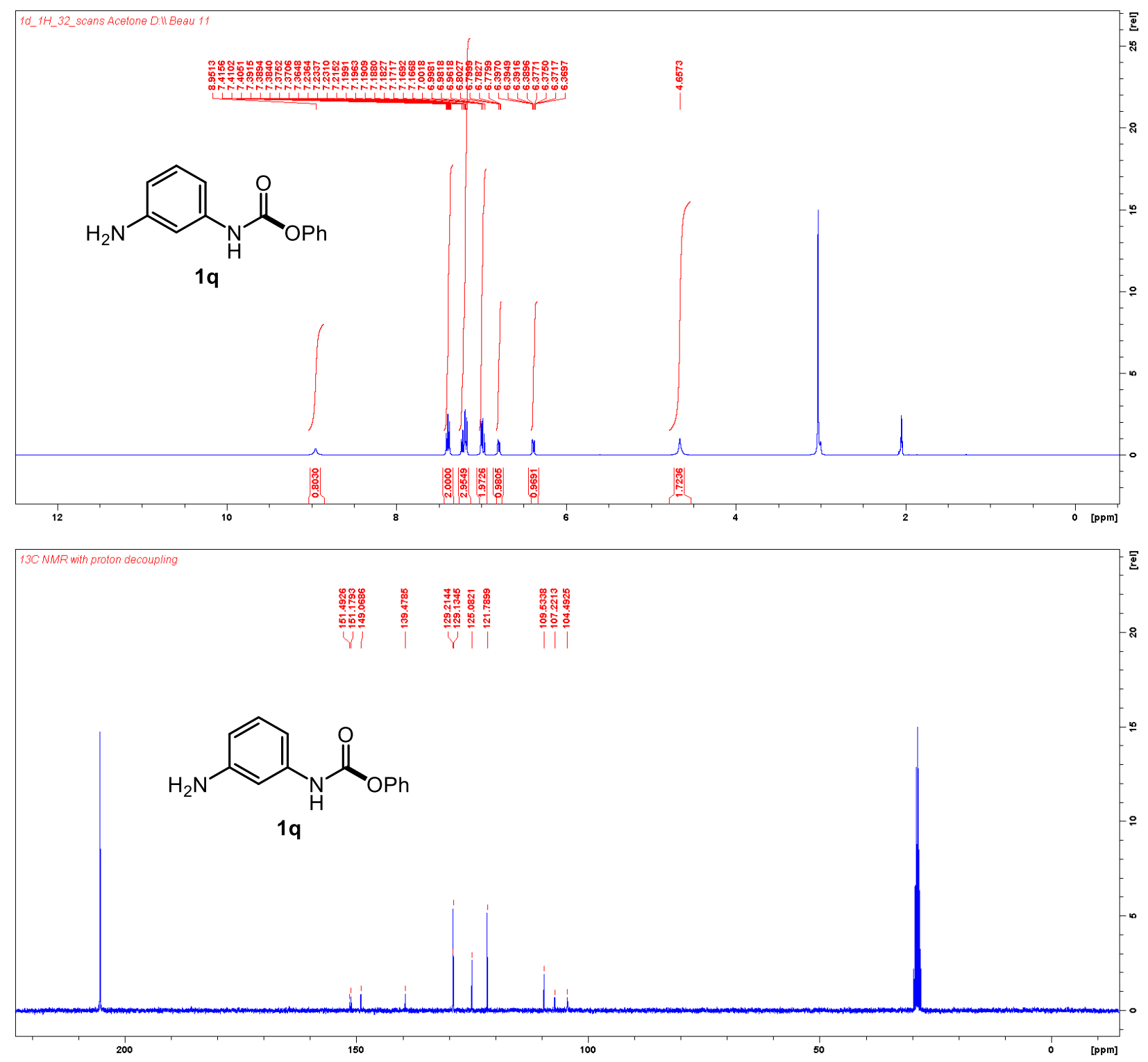

Proton: JD-10-62-3B Carbon: JD-10-62-3BBB 

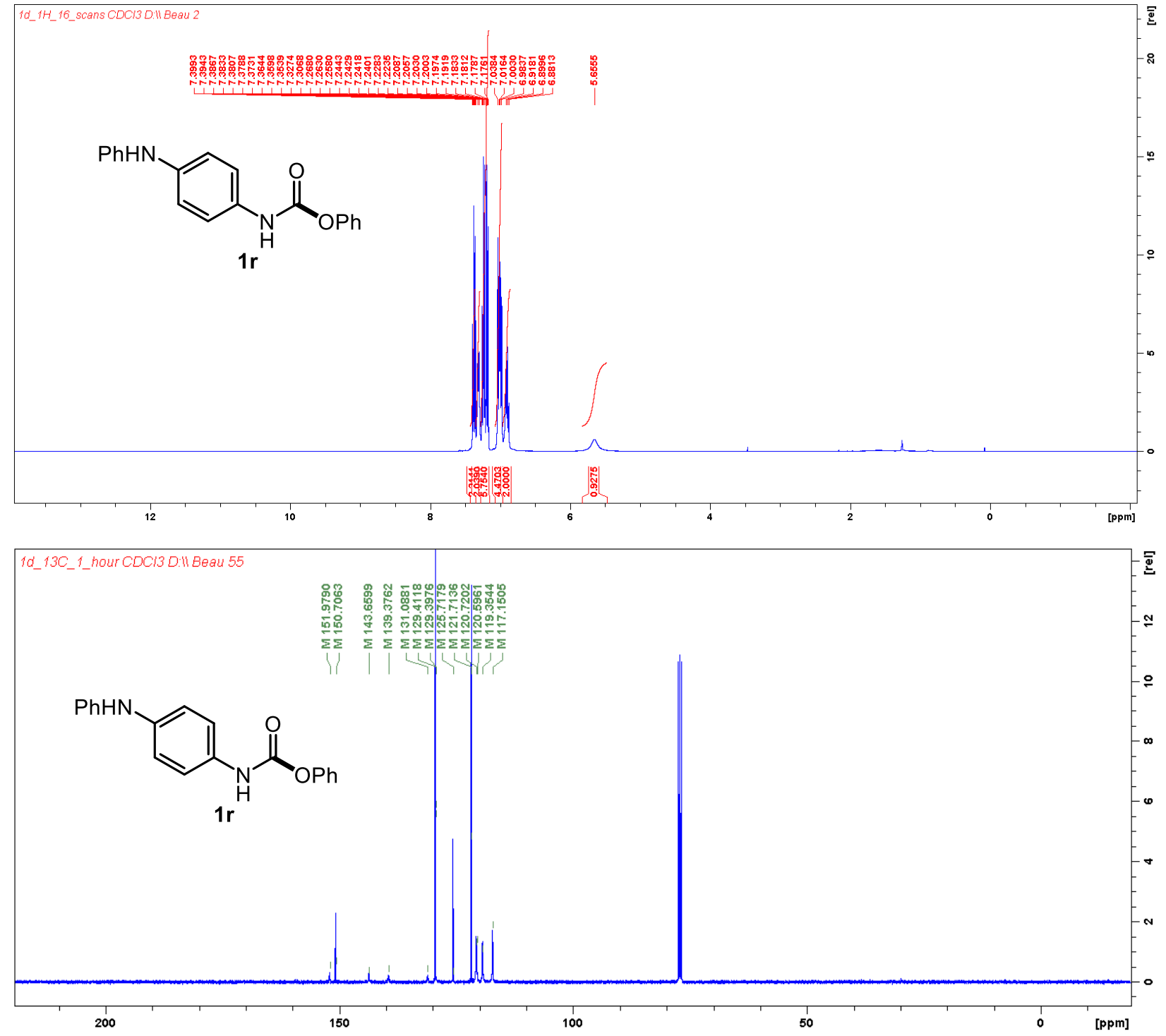

Proton: JD-13-1-1A Carbon: JD-13-1-2A 


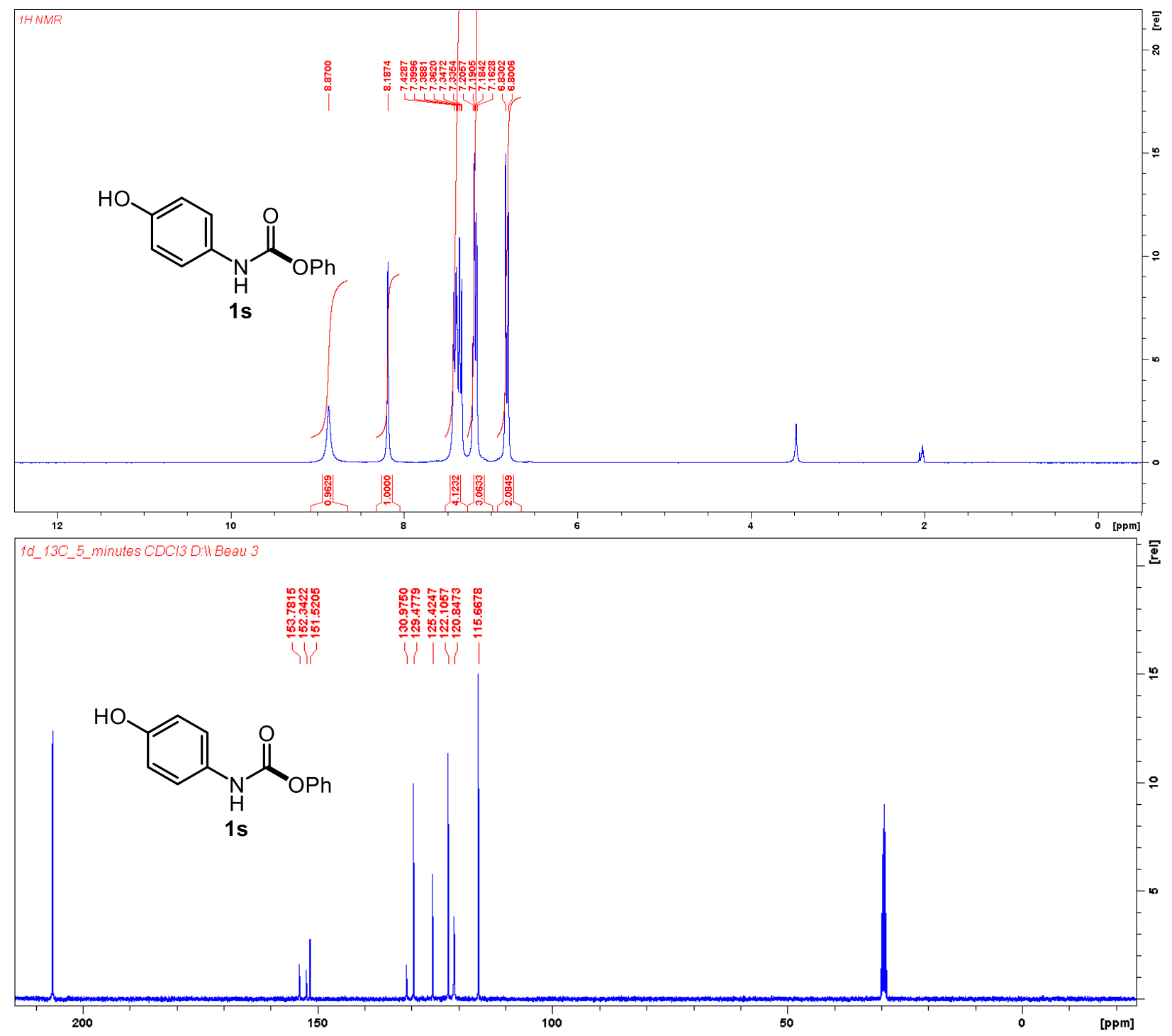

Proton: JD-13-2F Carbon: JD-13-1-1F 

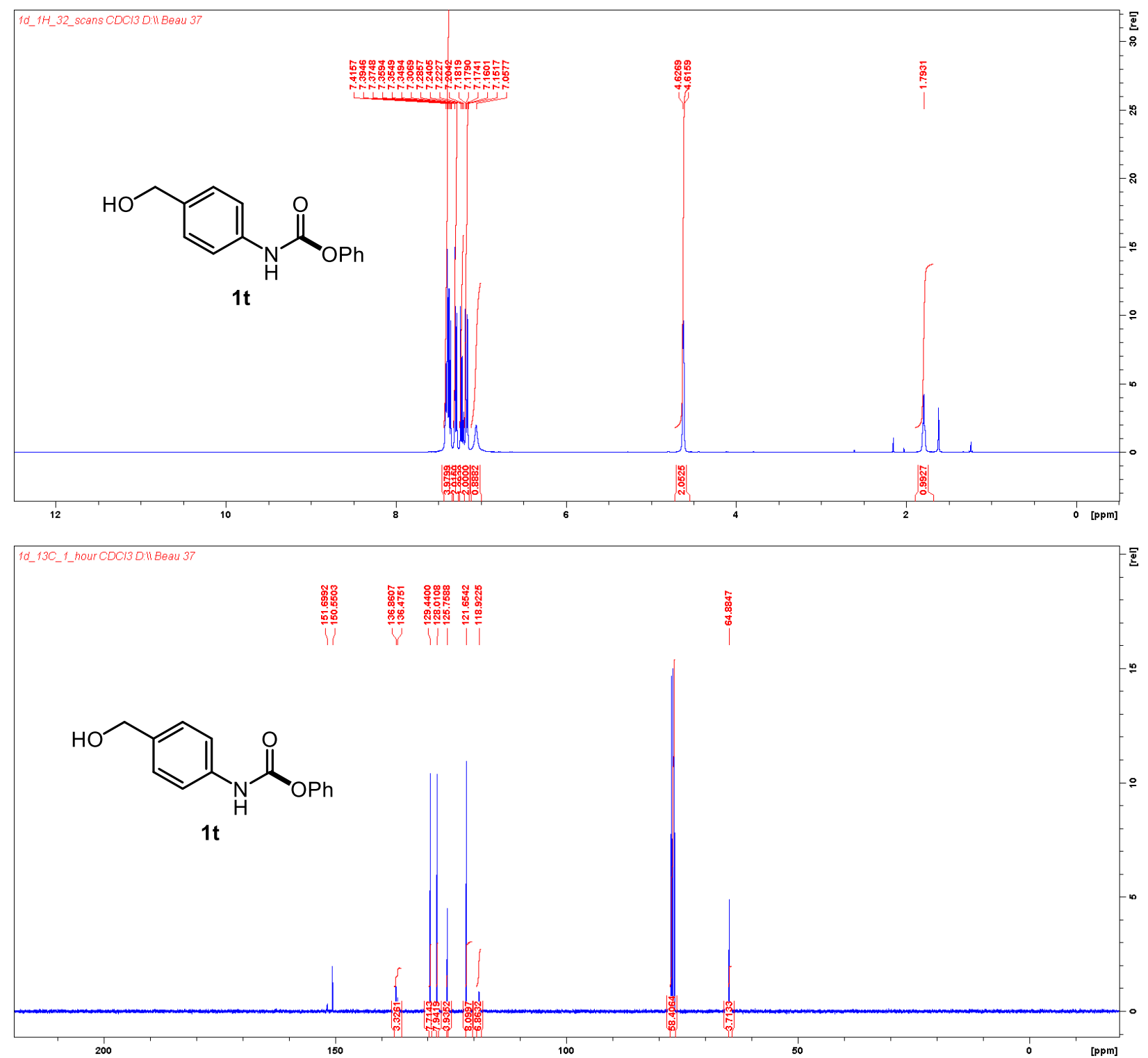

Proton: JD-10-48-2 Carbon: JD-10-48-2 

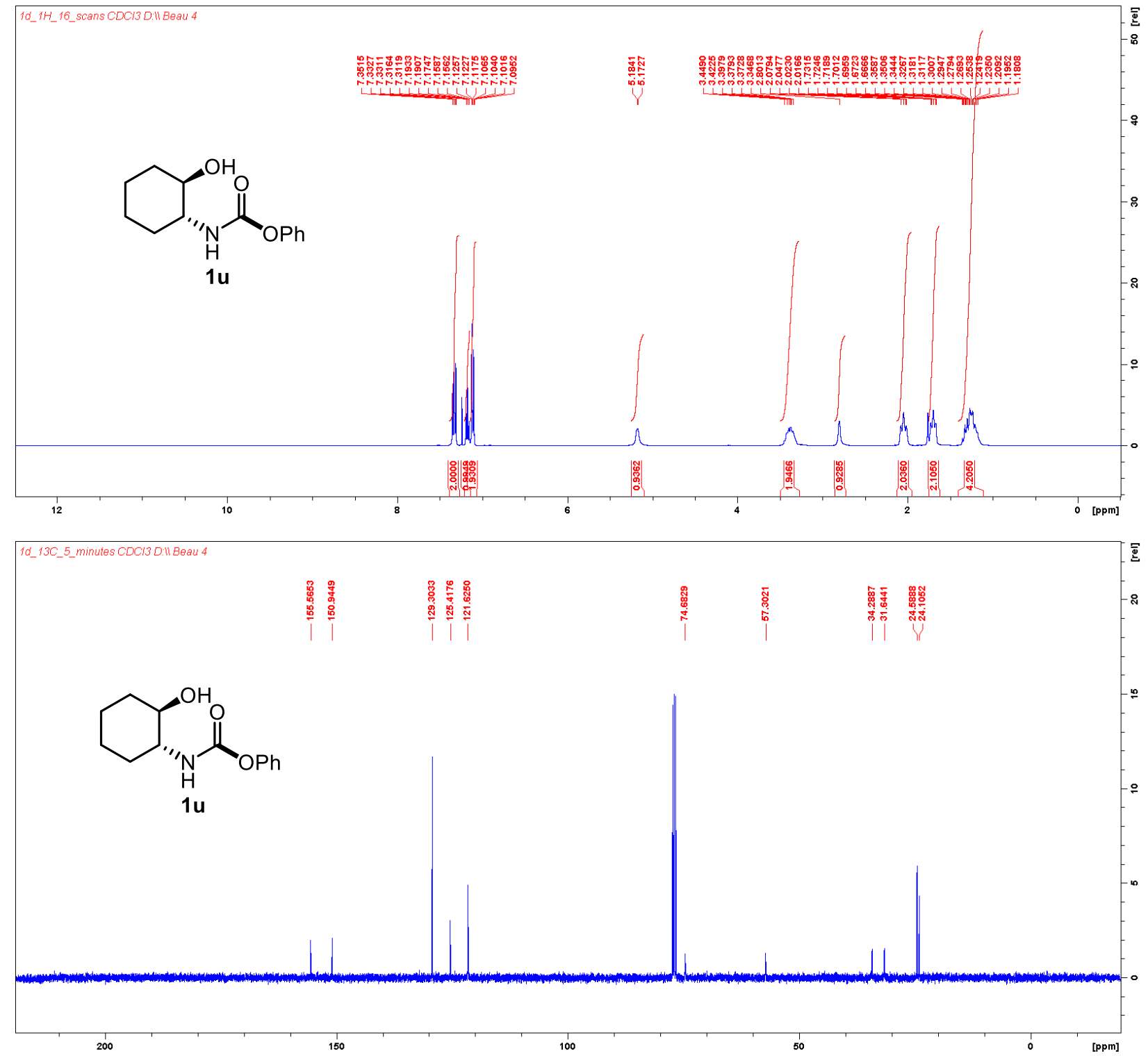

Proton: JD-13-1-1C Carbon: JD-13-1-1C 

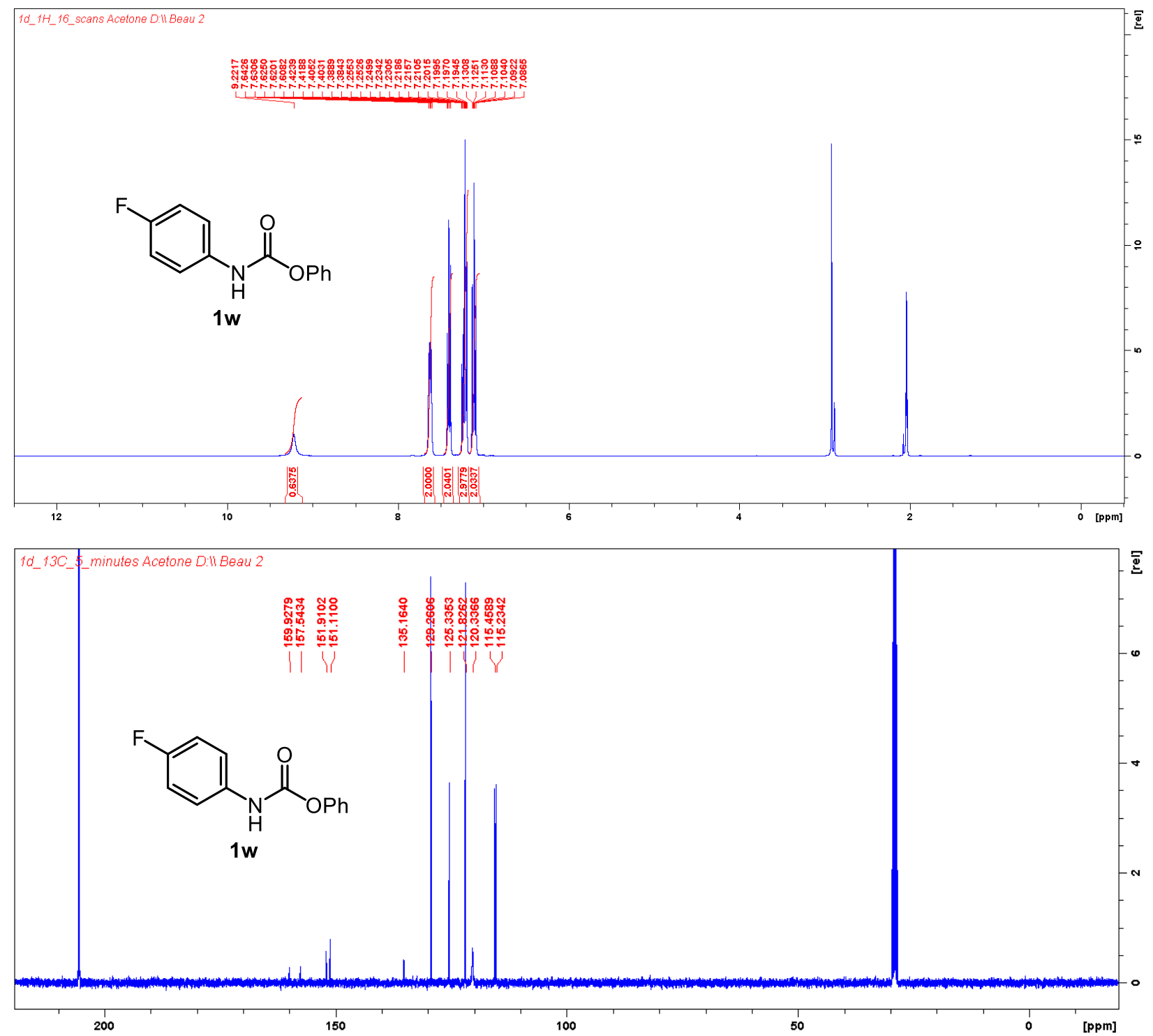

Proton: JD-12-58-4 Carbon: JD-12-58-4 

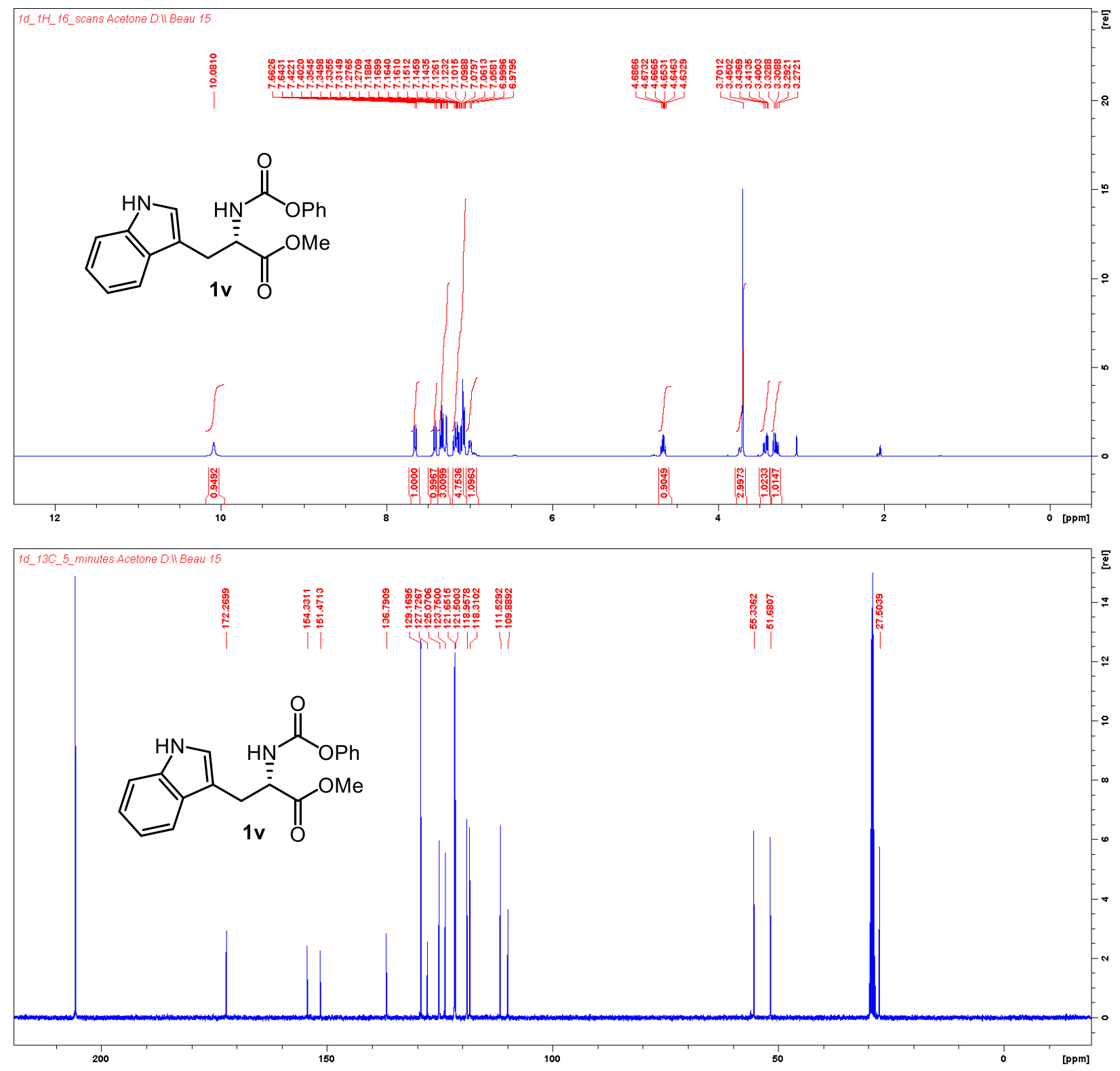

Proton:JD-13-3-3A Carbon: JD-13-3-3A 


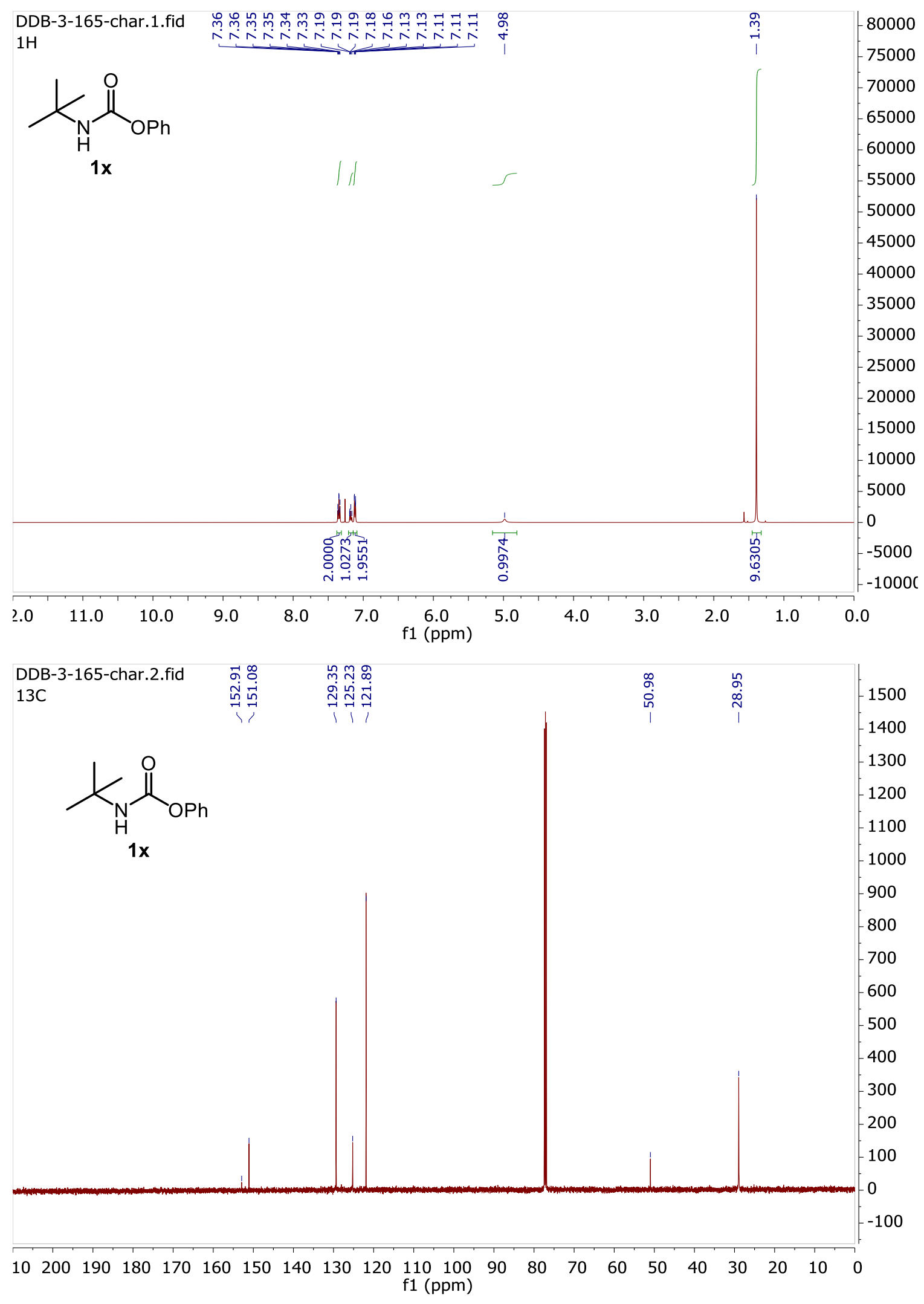



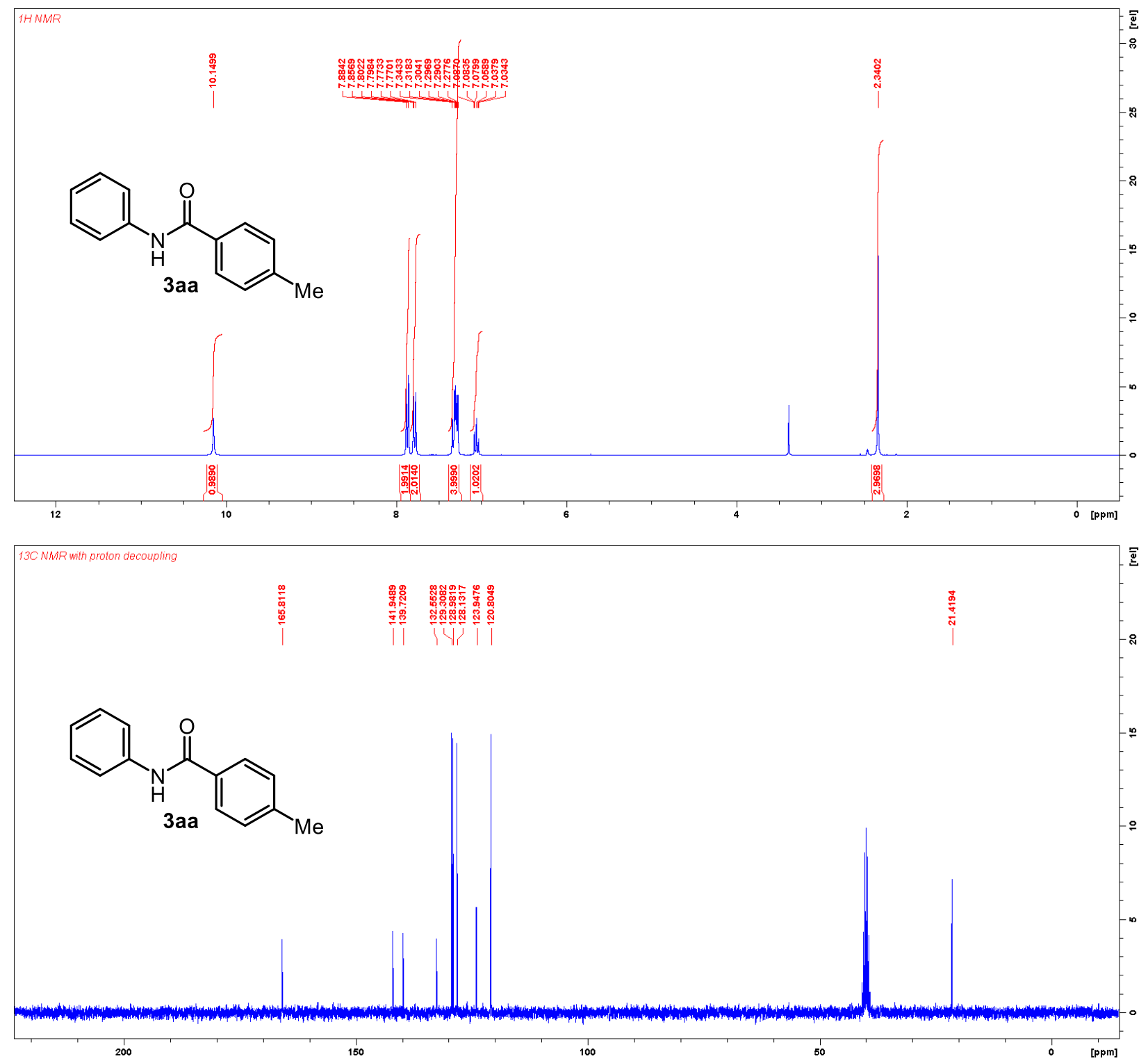

Proton: JD-9-10-2A Carbon: JD-9-10-2A 

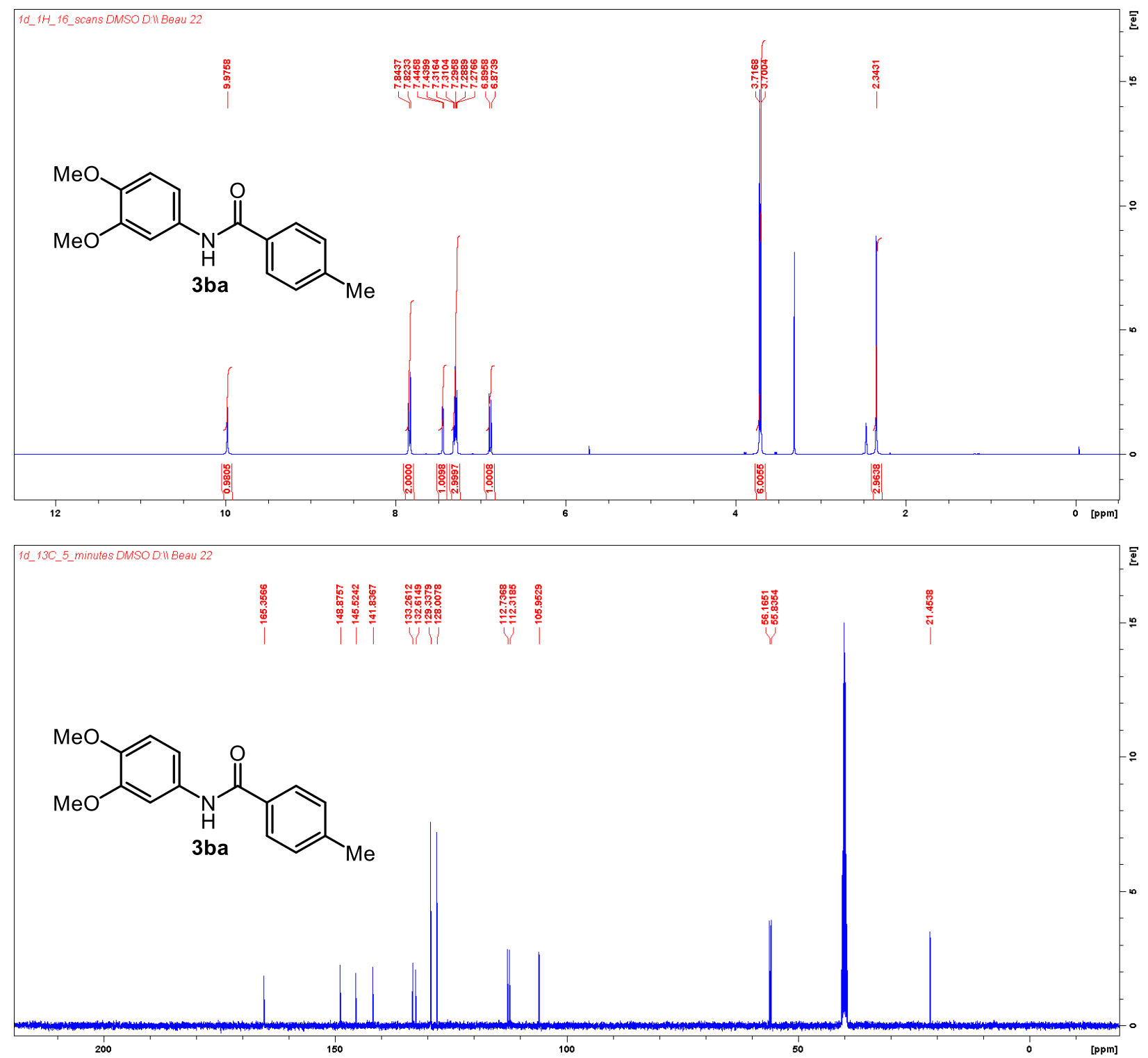

Proton: JD-9-12-10 Carbon: JD-9-12-11 

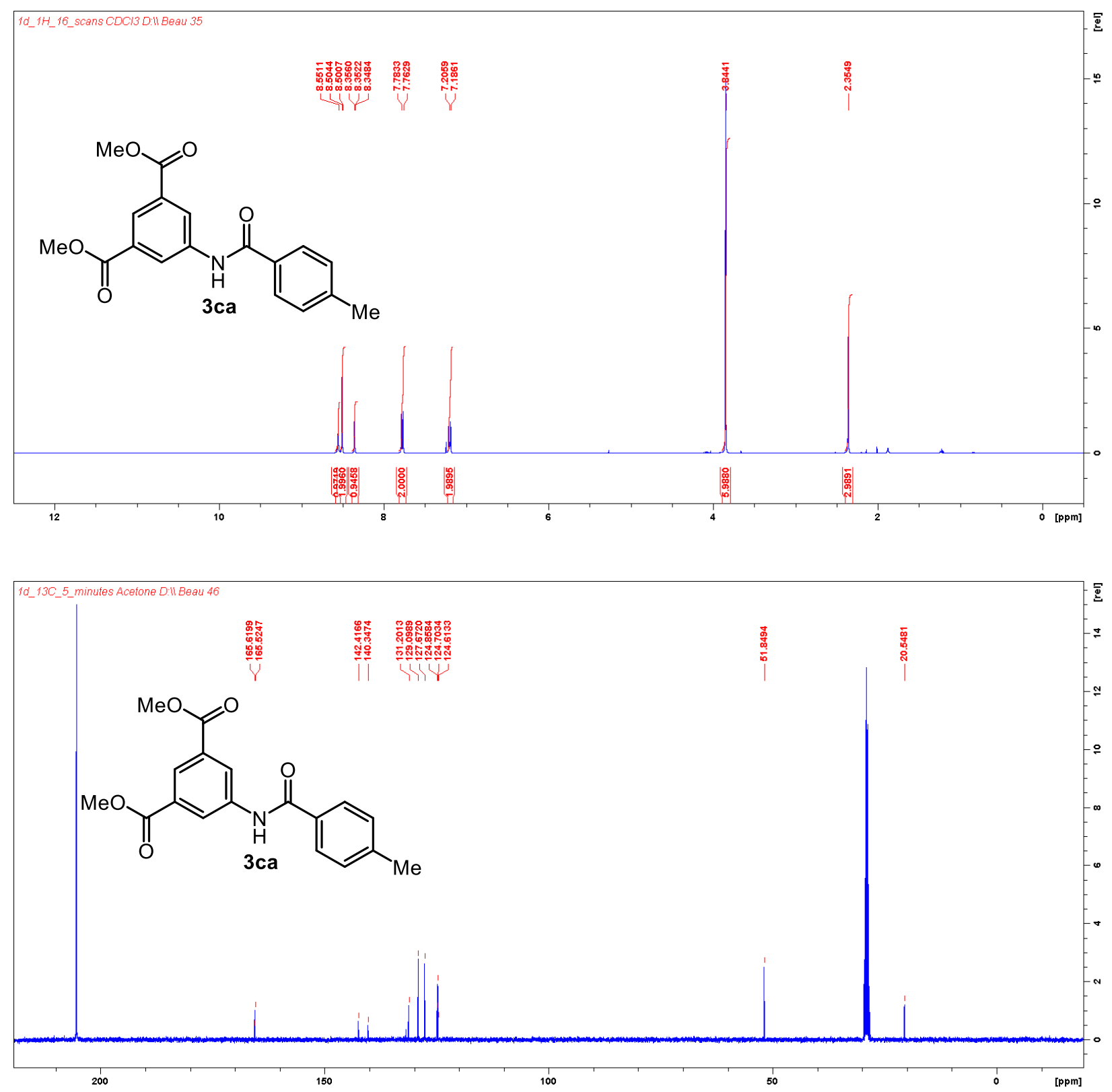

Proton: JD-9-22-1 Carbon: JD-9-22-2 


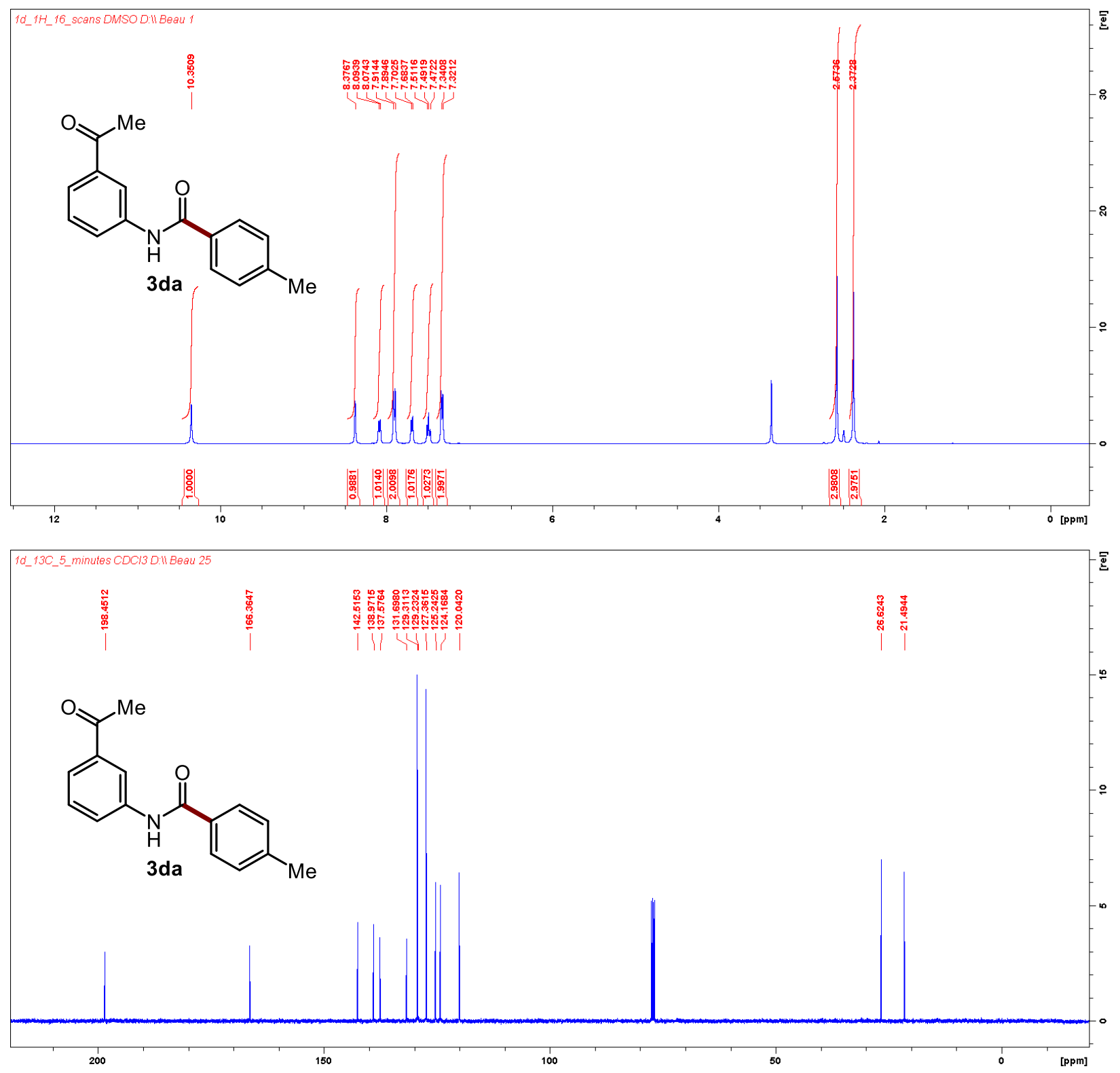

Proton: JD-10-2-3_2 Carbon: JD-10-21-3

S87 

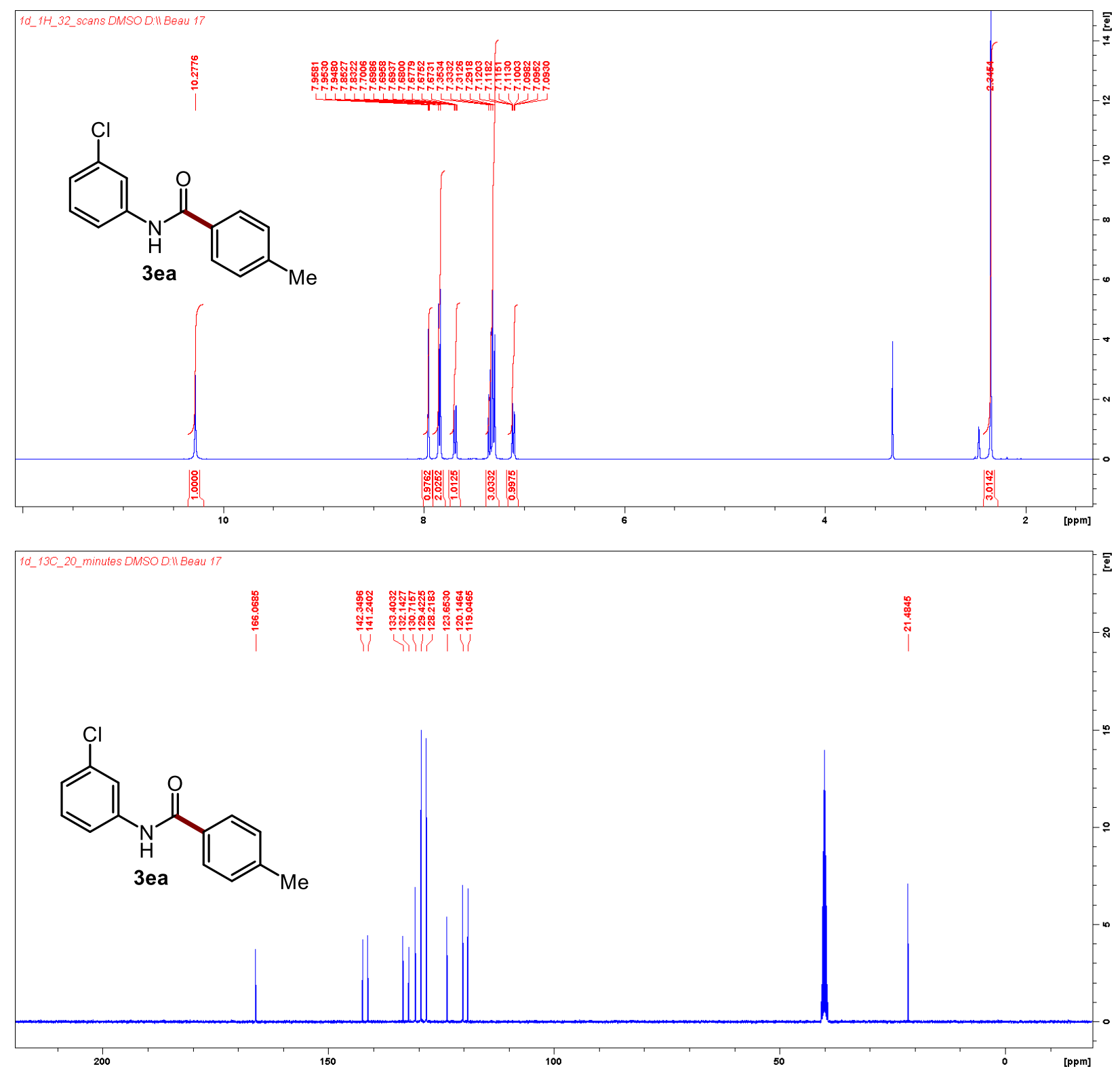

Proton: JD-10-10-2 Carbon: JD-10-10-2 

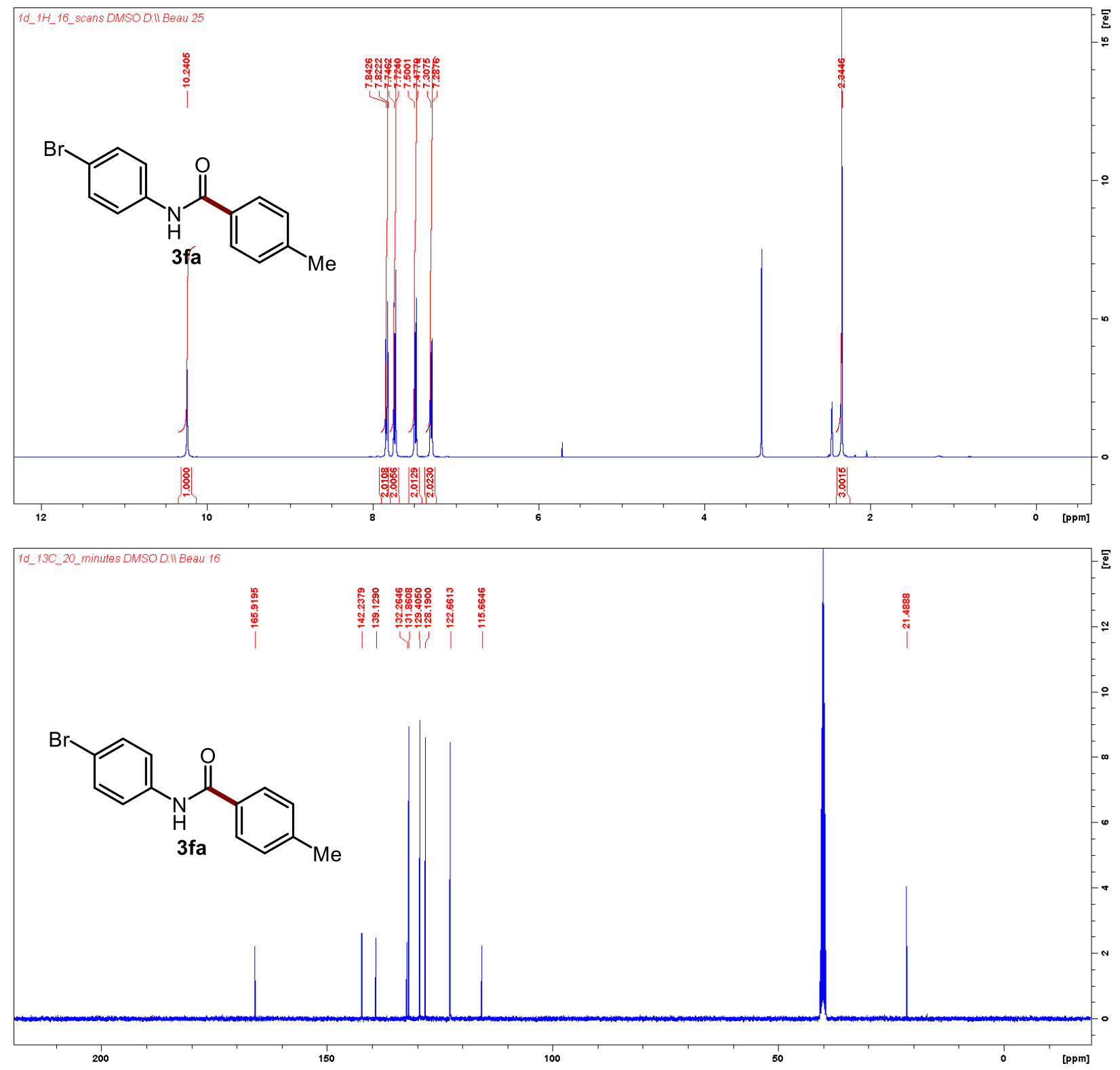

Proton: JD-10-5-2 Carbon: JD-10-5-2 

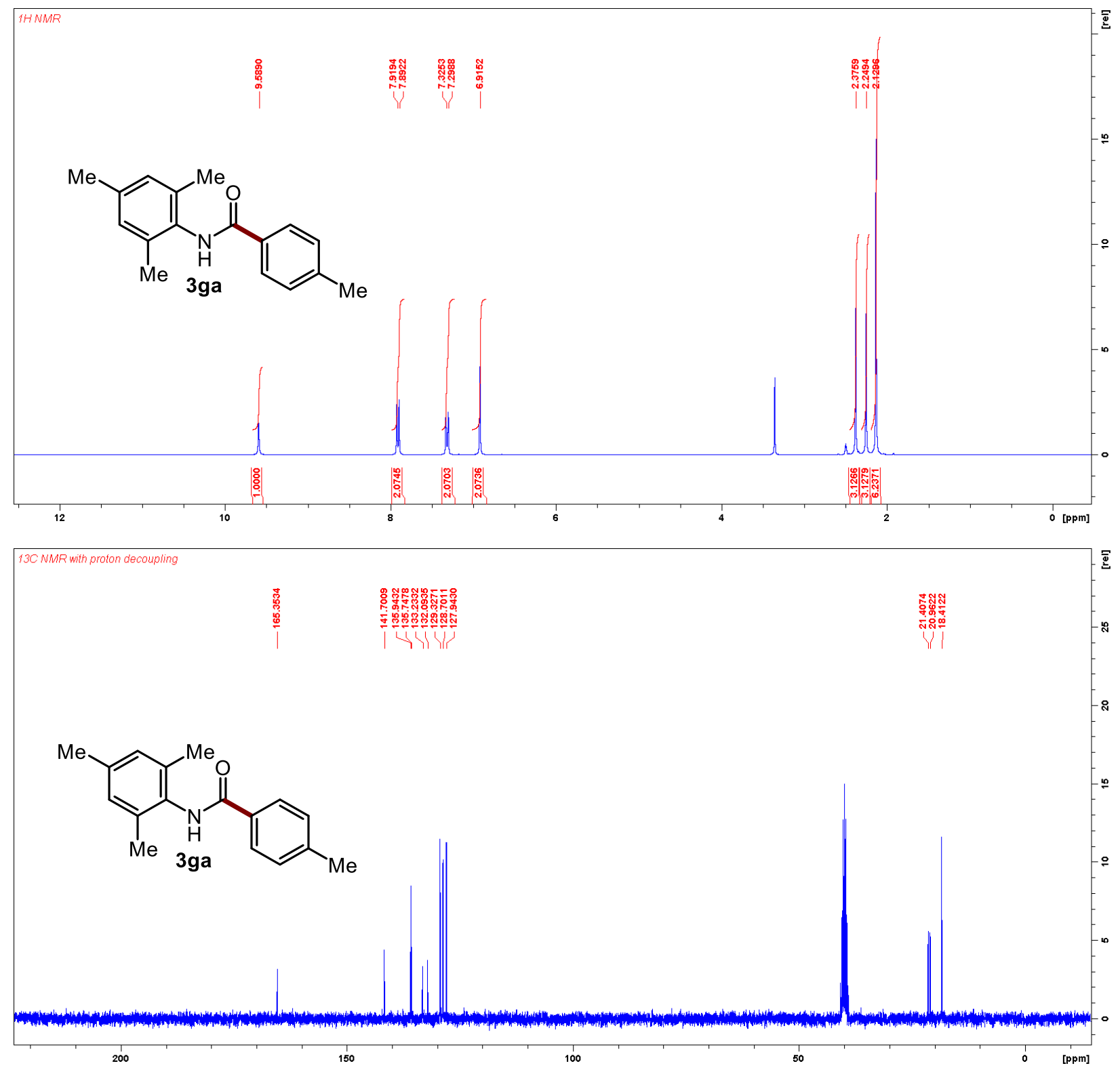

Proton: JD-10-21-2 Carbon: JD-10-21-2 

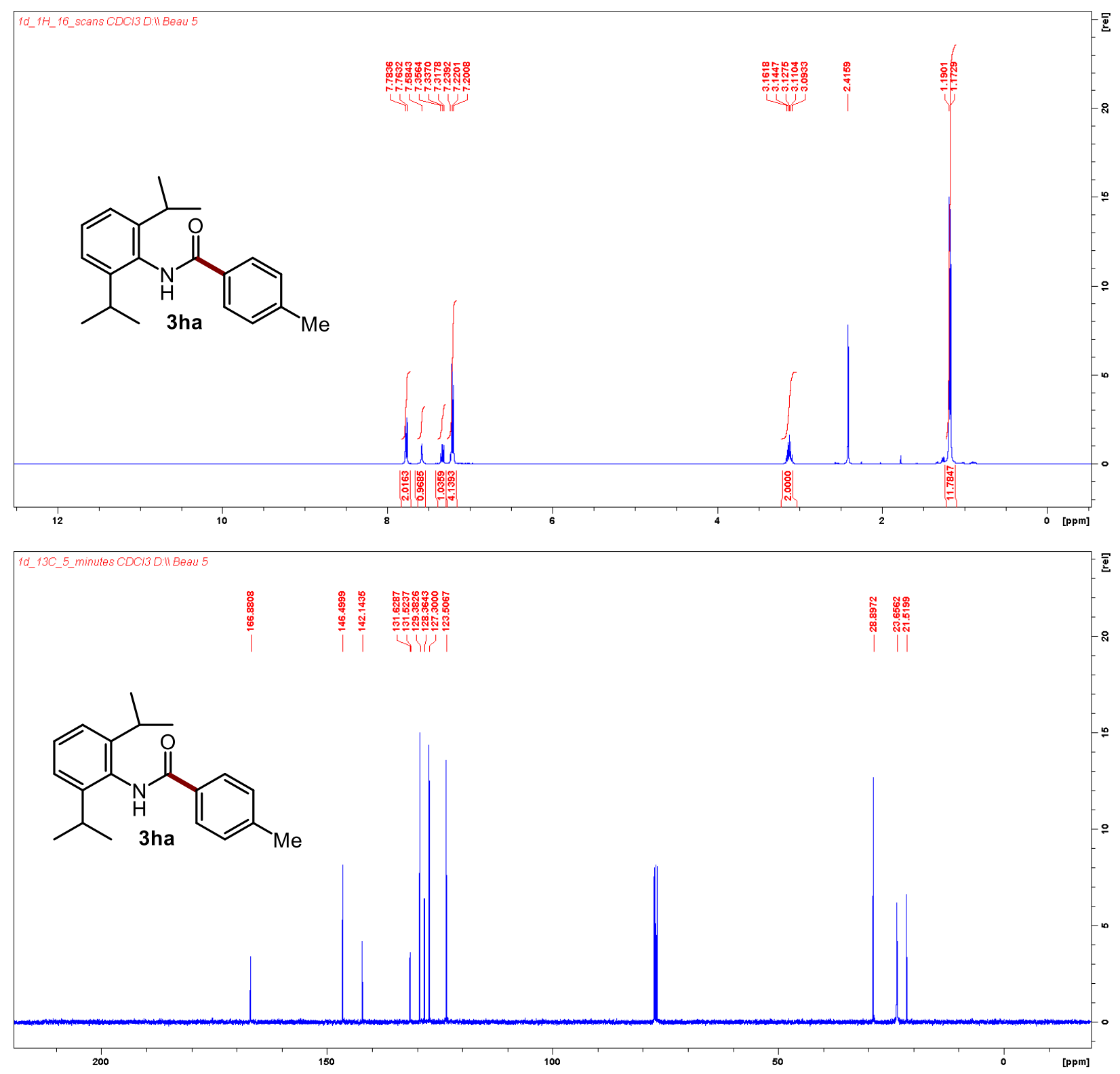

Proton: JD-11-4-1A Carbon: JD-11-4-1A 

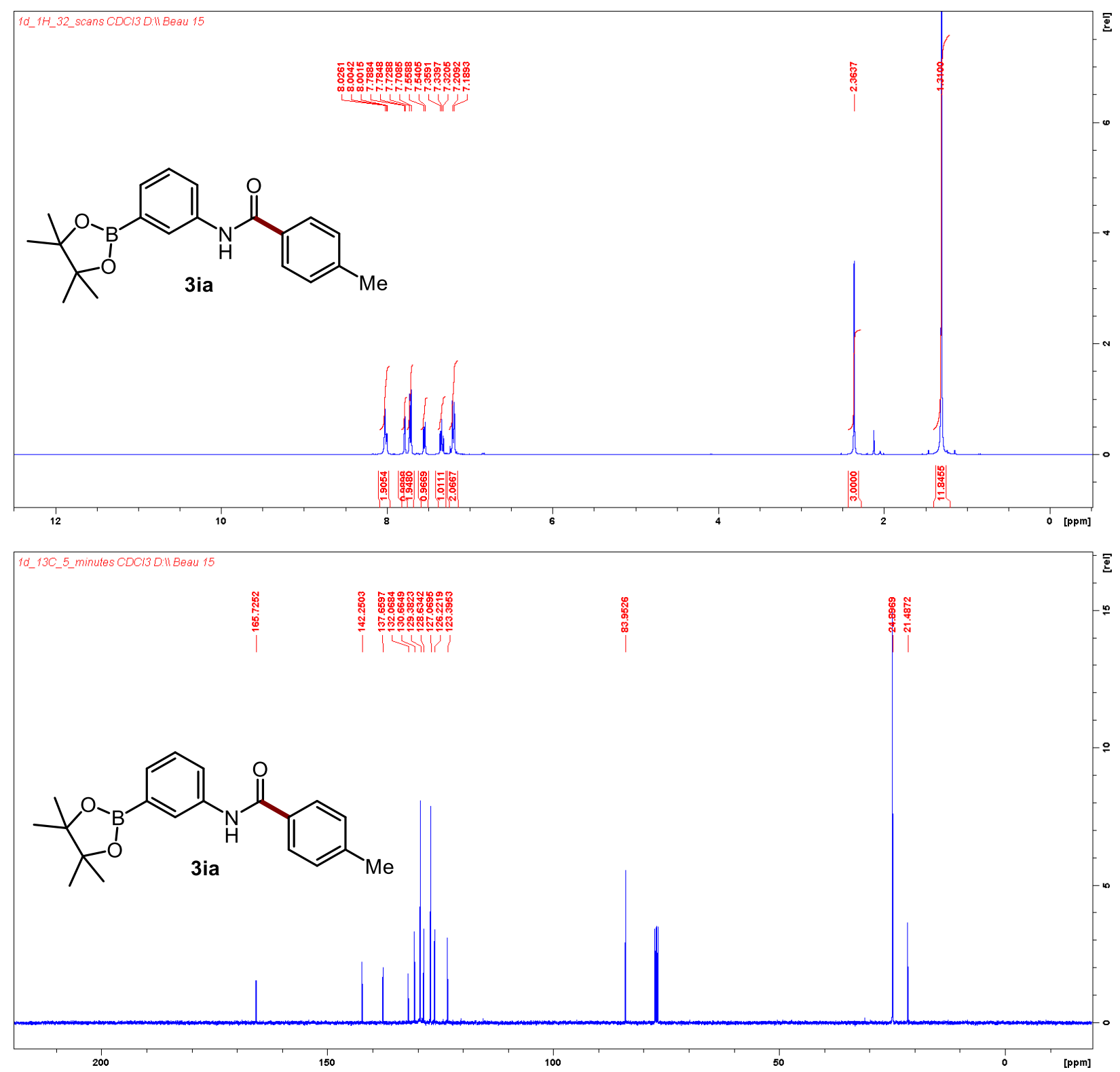

Proton: JD-10-96-5 Carbon: JD-10-96-5 

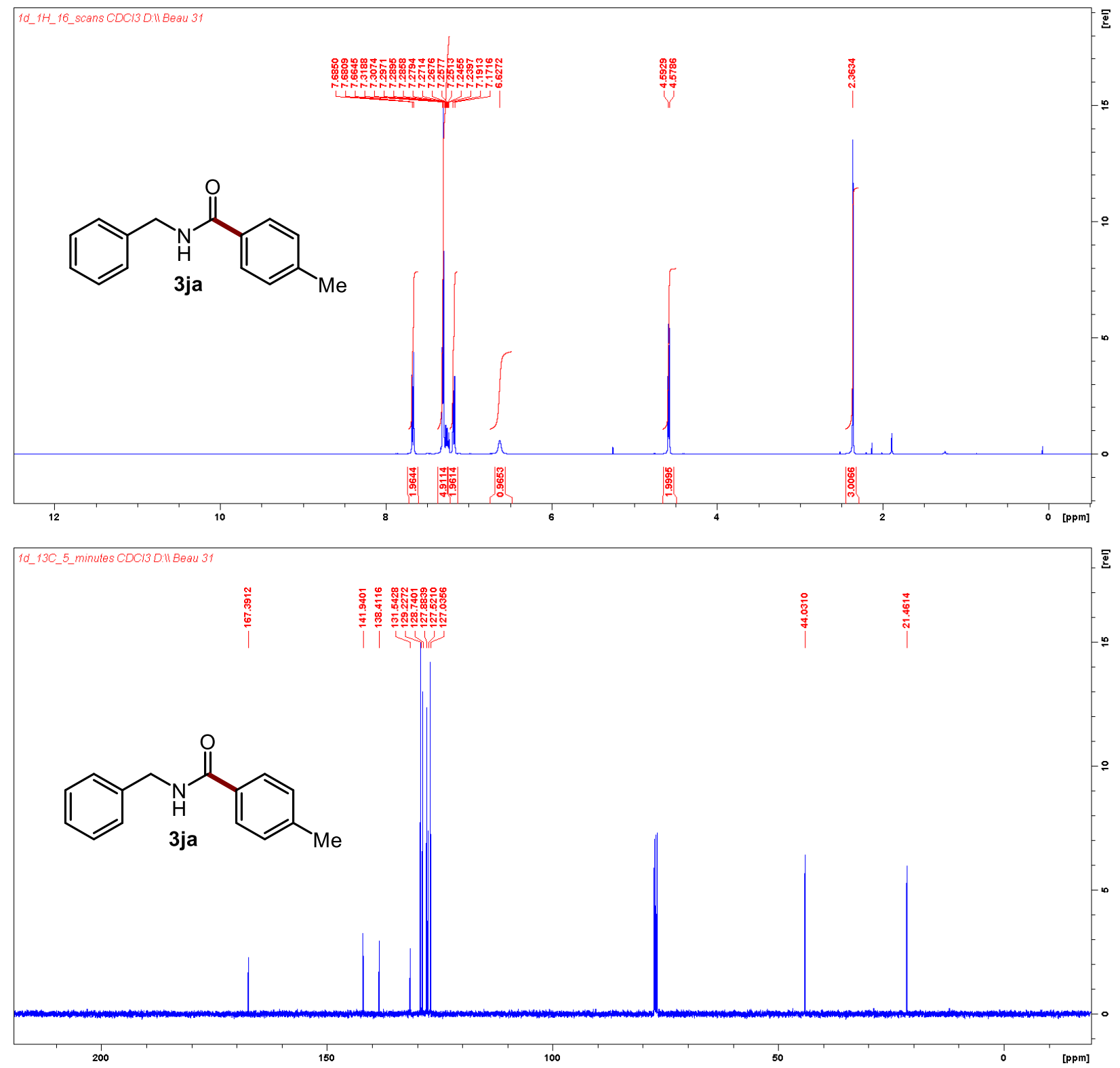

Proton: JD-9-4-4B Carbon: JD-9-4-4B 

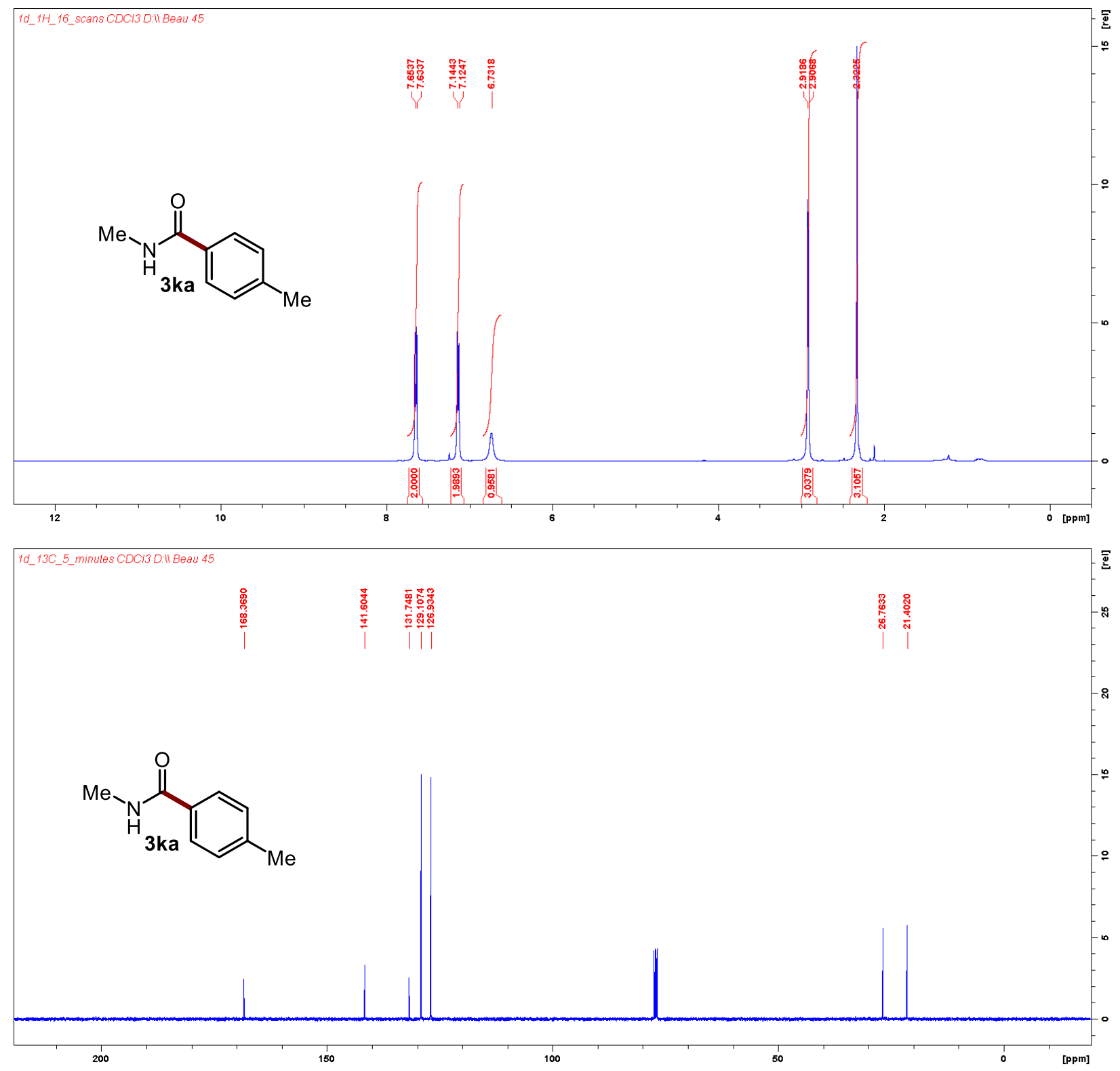

Proton: JD-10-65-2A Carbon: JD-10-65-2A 

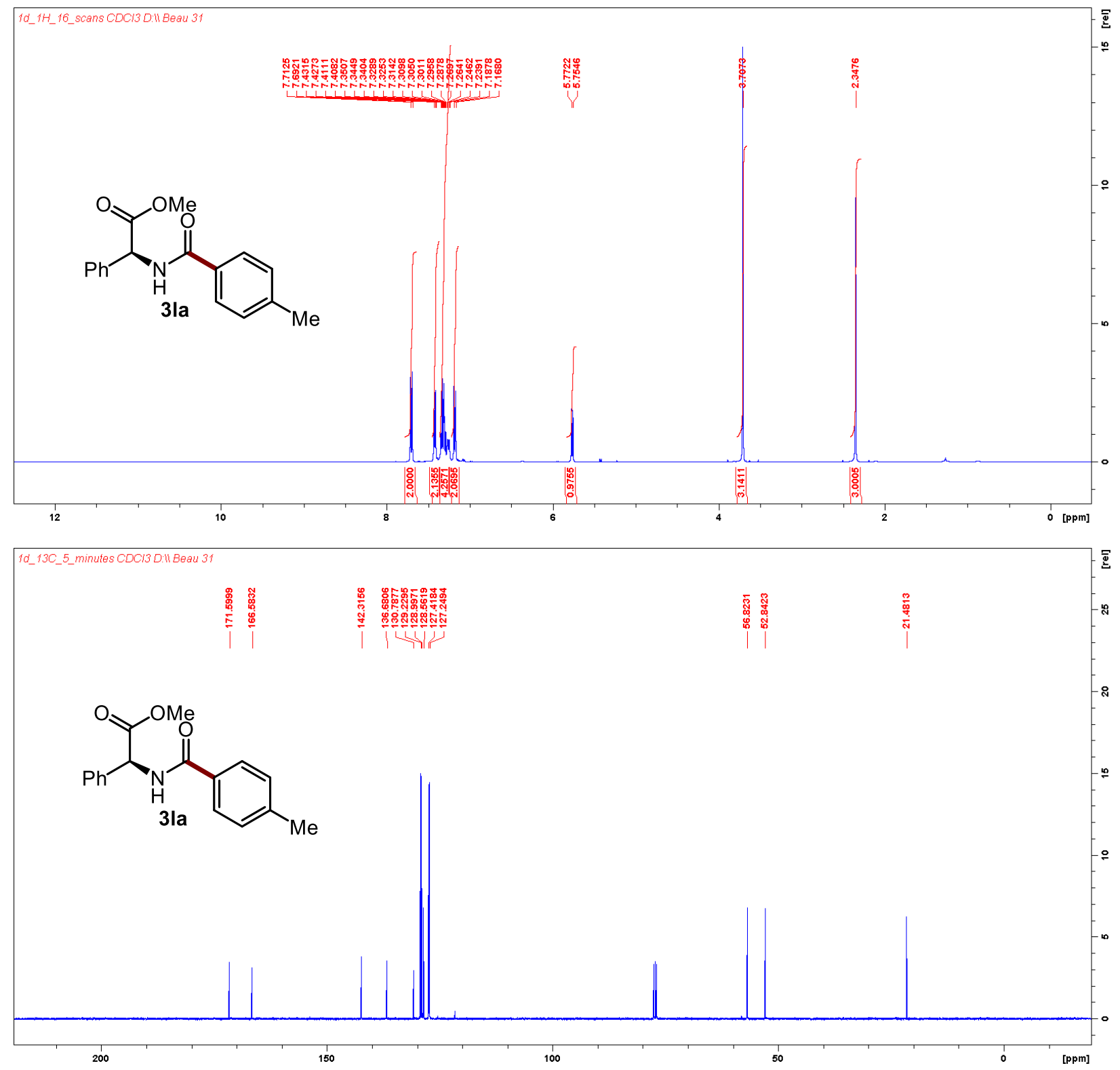

Proton: JD-9-23-3 Carbon: JD-9-23-3 

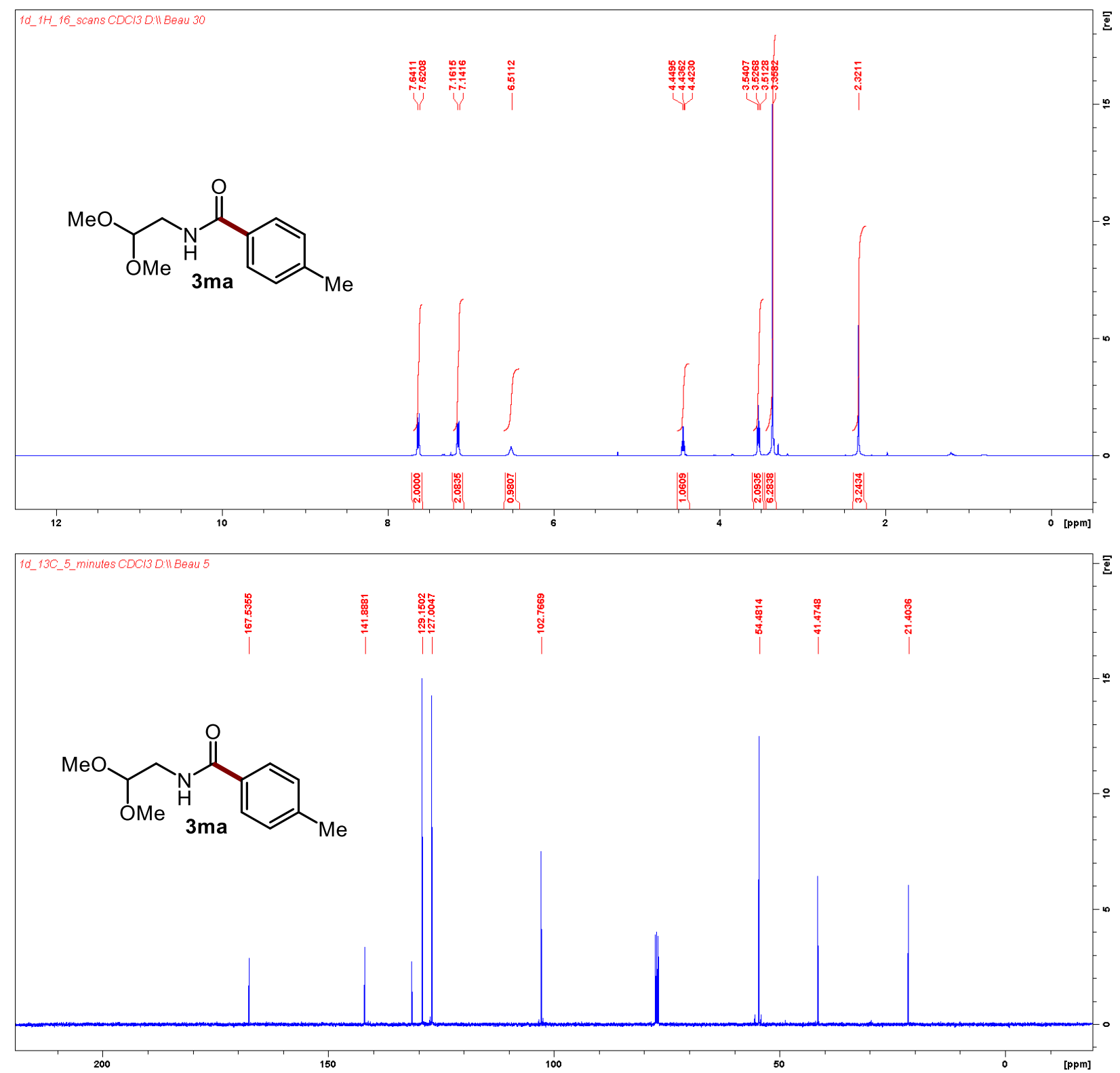

Proton: JD-10-84-1A Carbon: JD-10-84-2A 

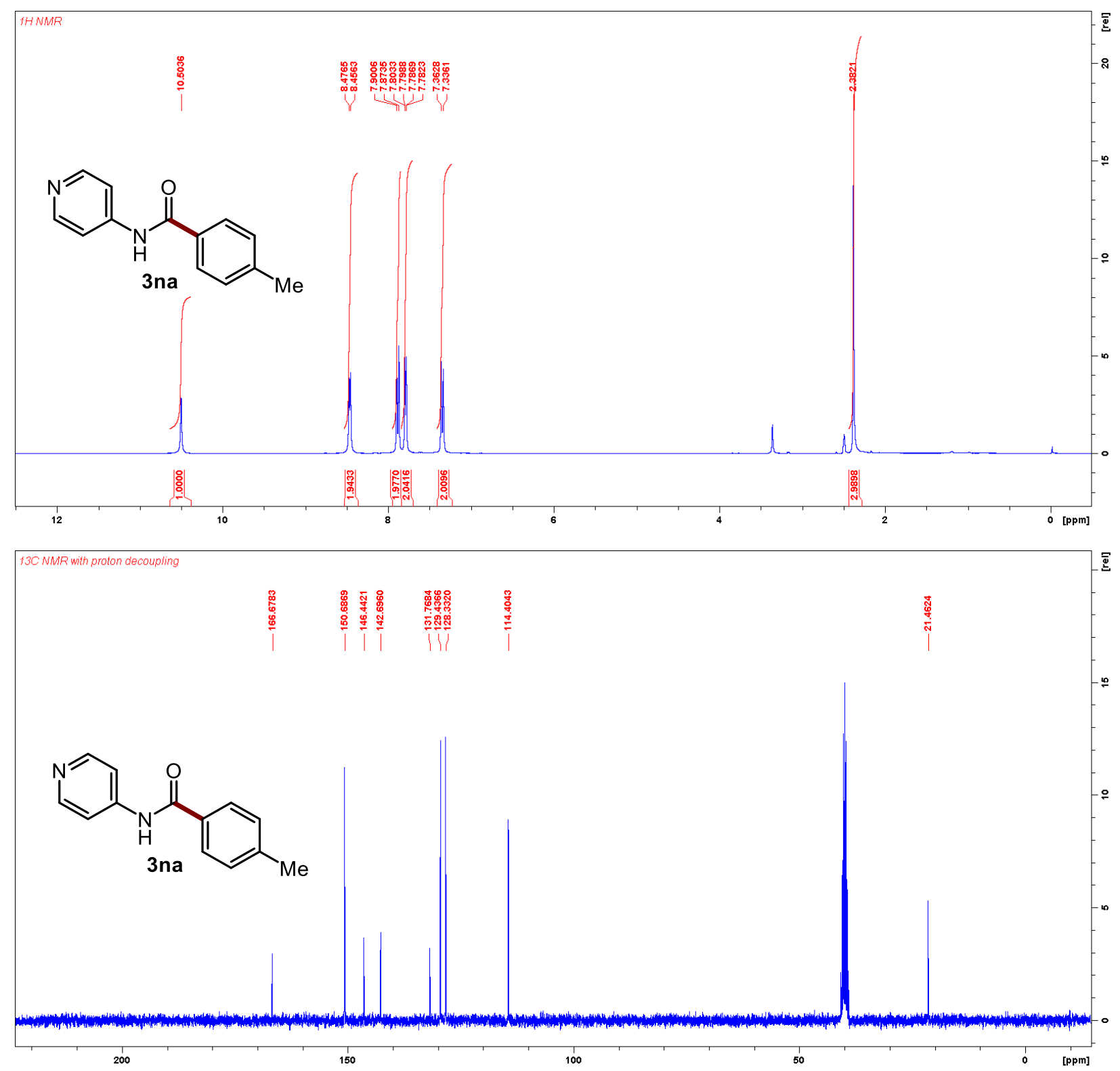

Proton: JD-11-27-2C Carbon: JD-11-27-2C 

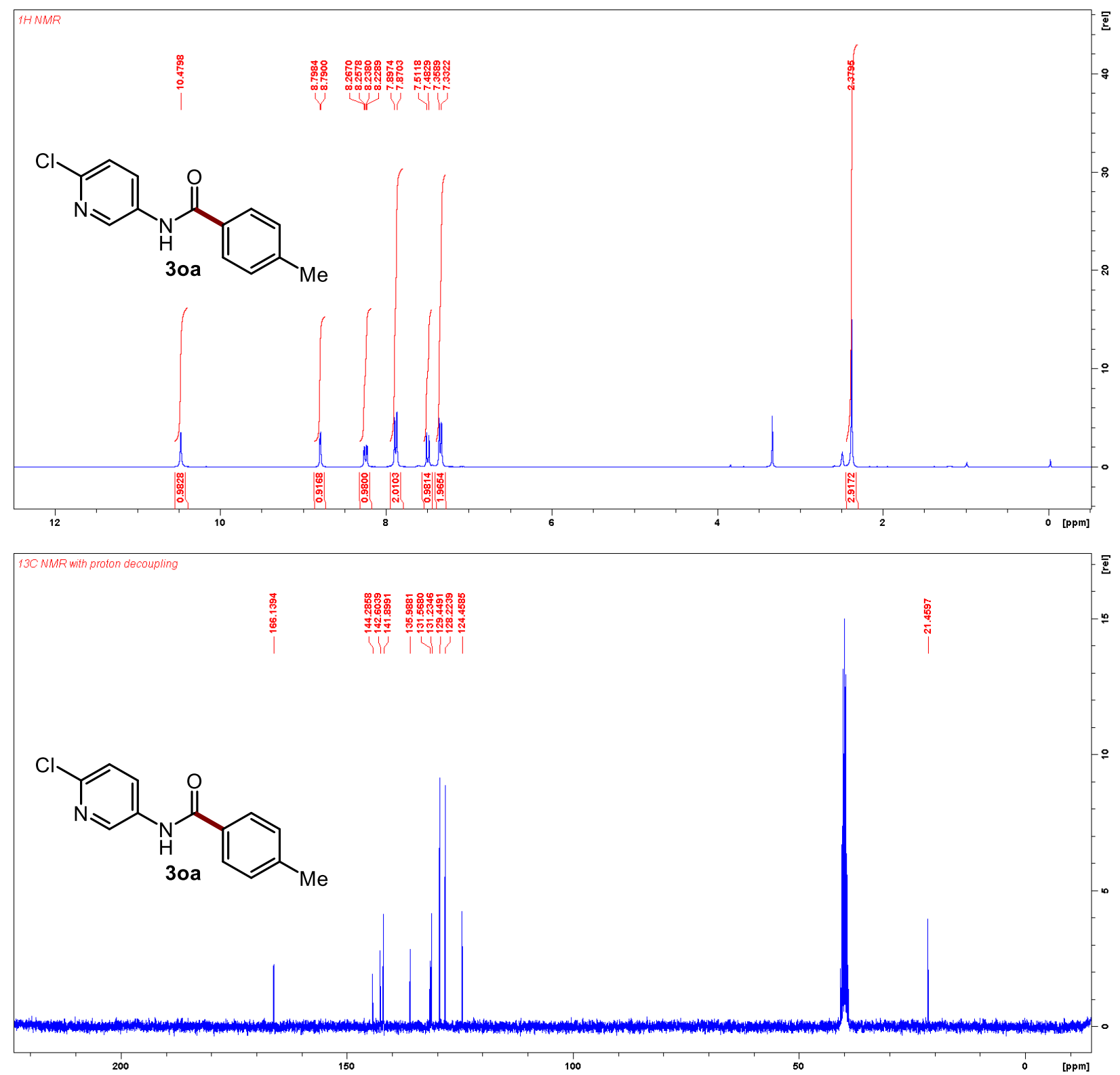

Proton: JD-11-28-2C Carbon: JD-11-28-2C 

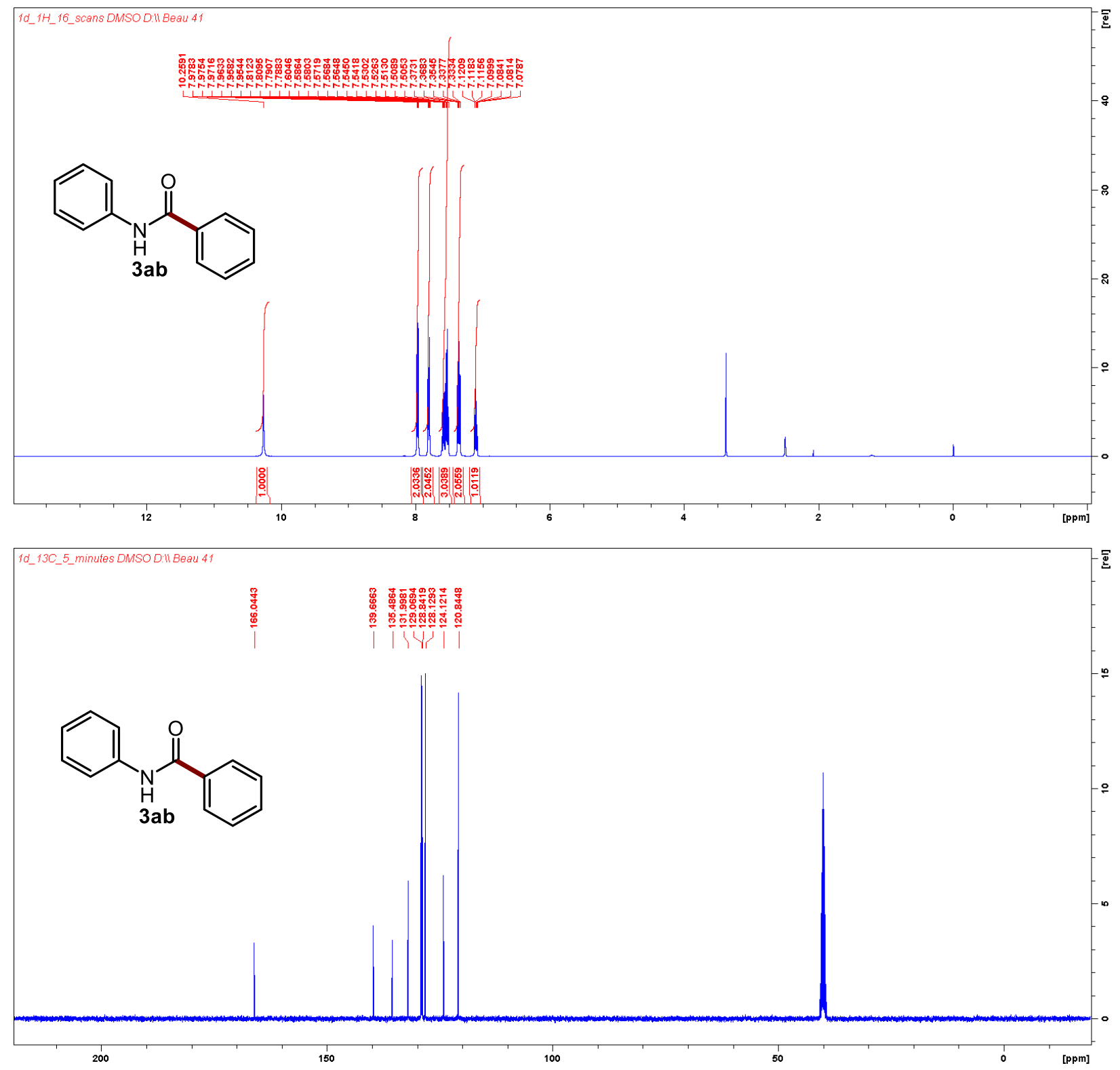

Proton: JD-9-27-2 Carbon: JD-9-27-2 

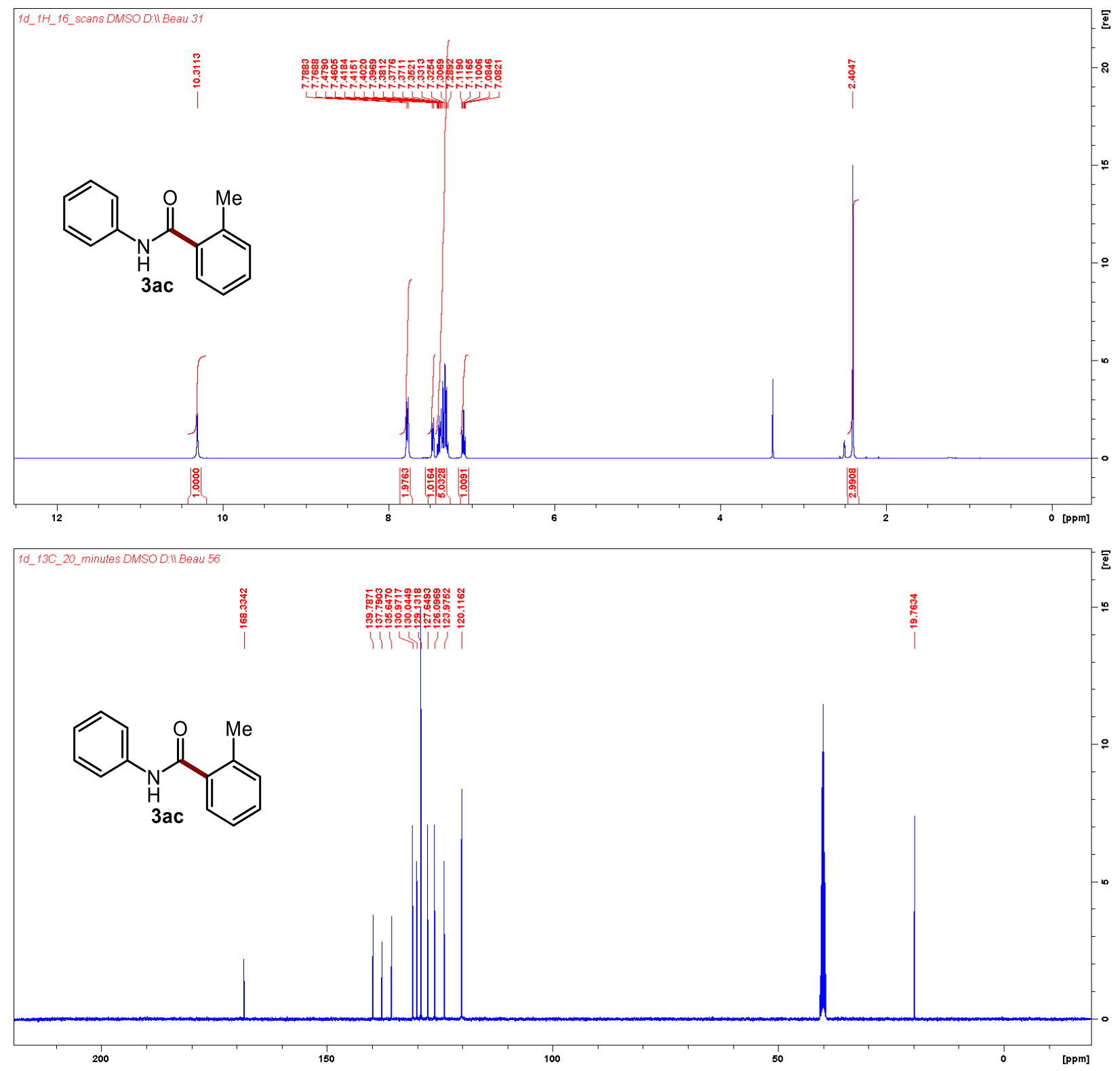

Proton: JD-10-15-2 Carbon: JD-10-15-3 

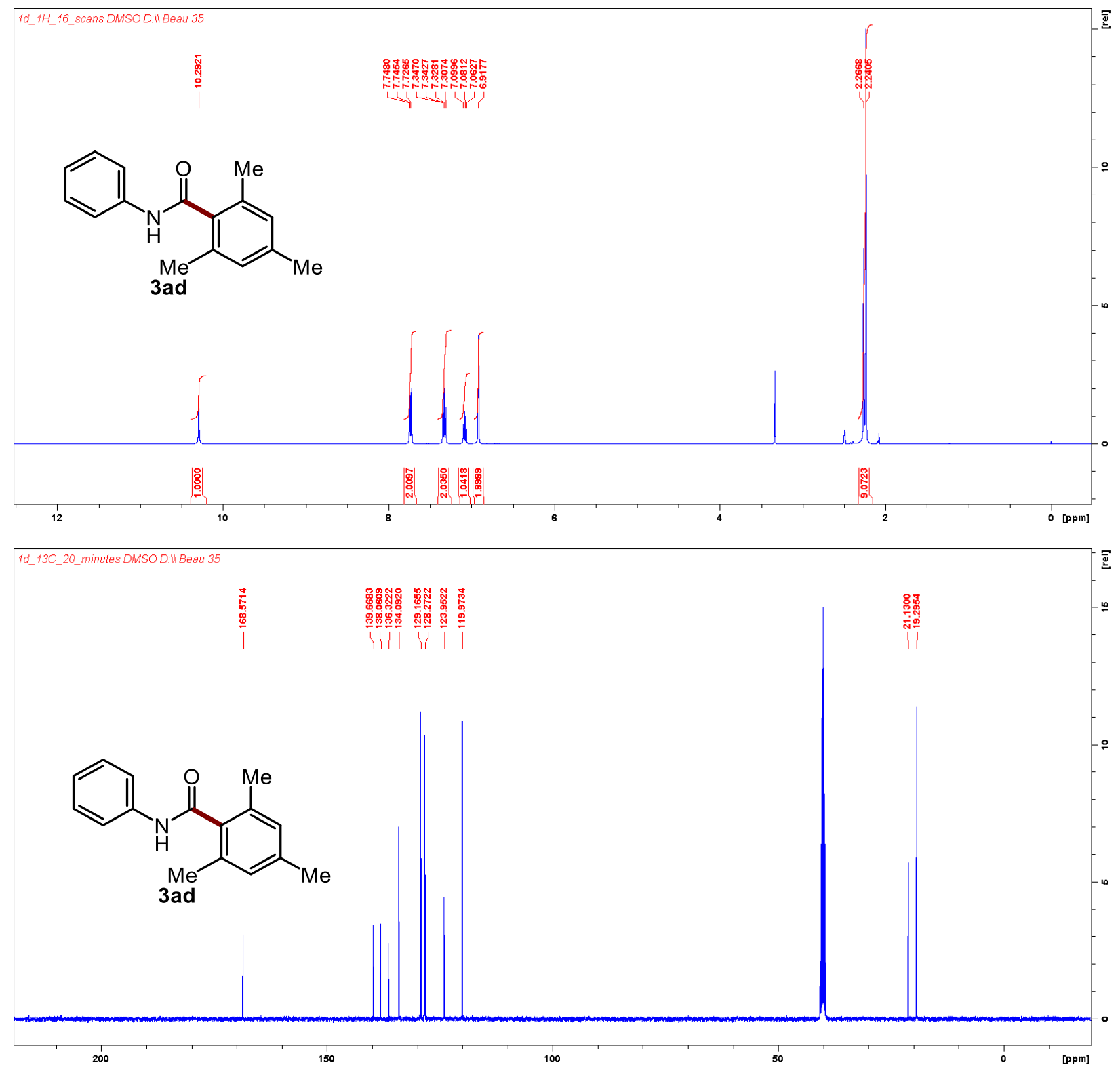

Proton: JD-10-42-2 Carbon: JD-10-42-2 

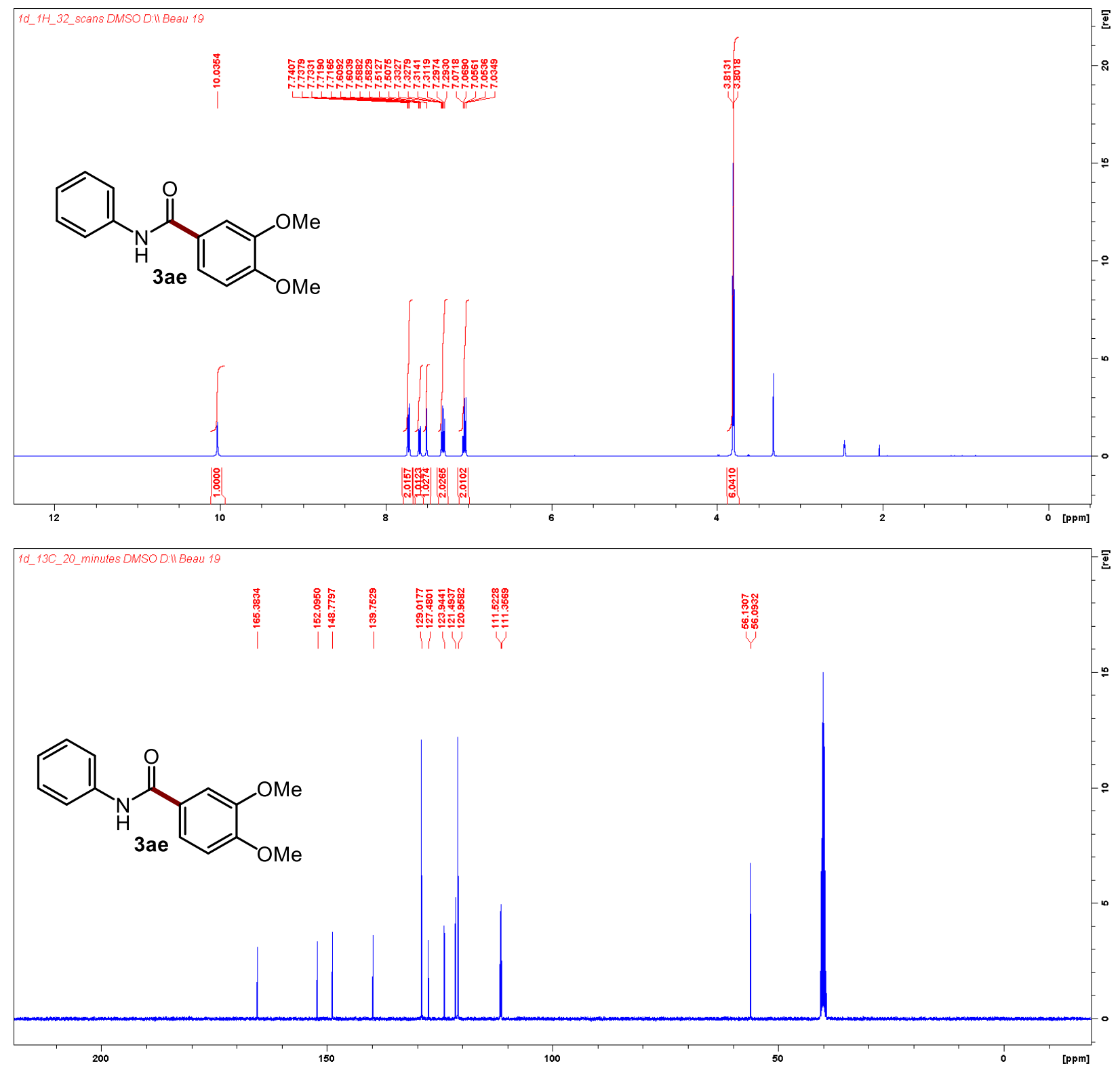

Proton: JD-10-12-1_2 Carbon: JD-10-12-1 

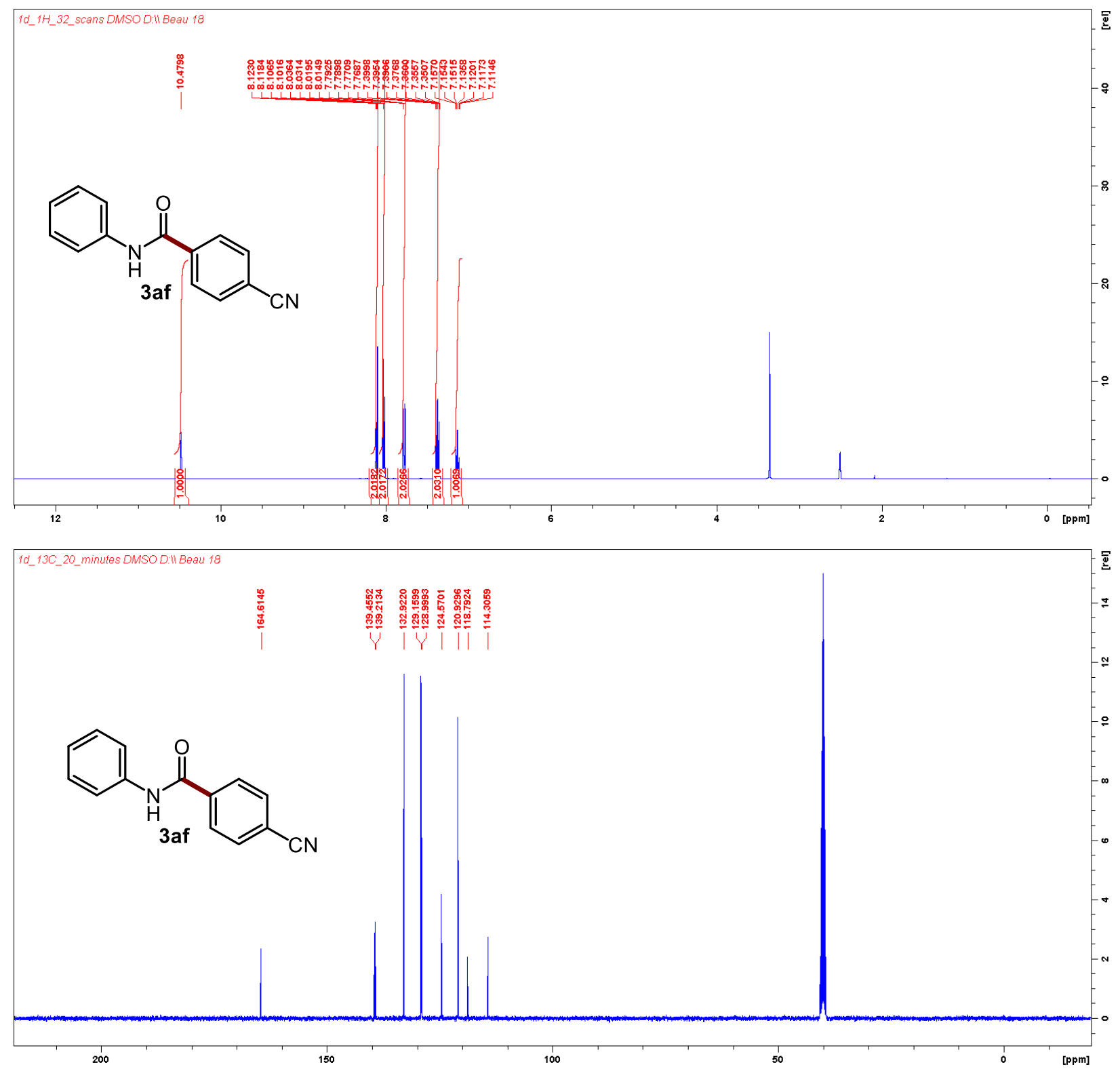

Proton: JD-10-11-2 Carbon: JD-10-11-2 

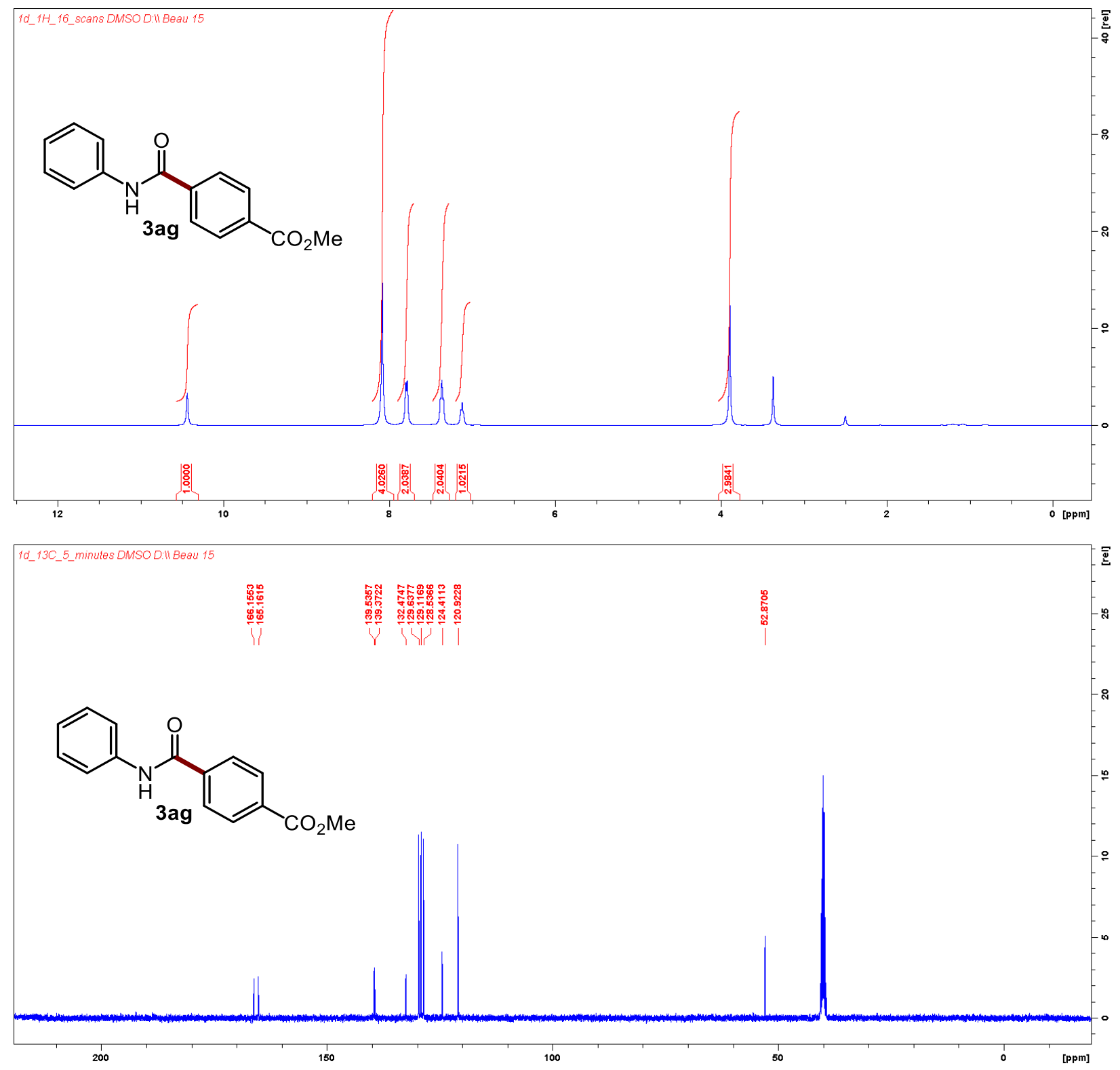

Proton: JD-10-70-2B Carbon: JD-10-70-2B 

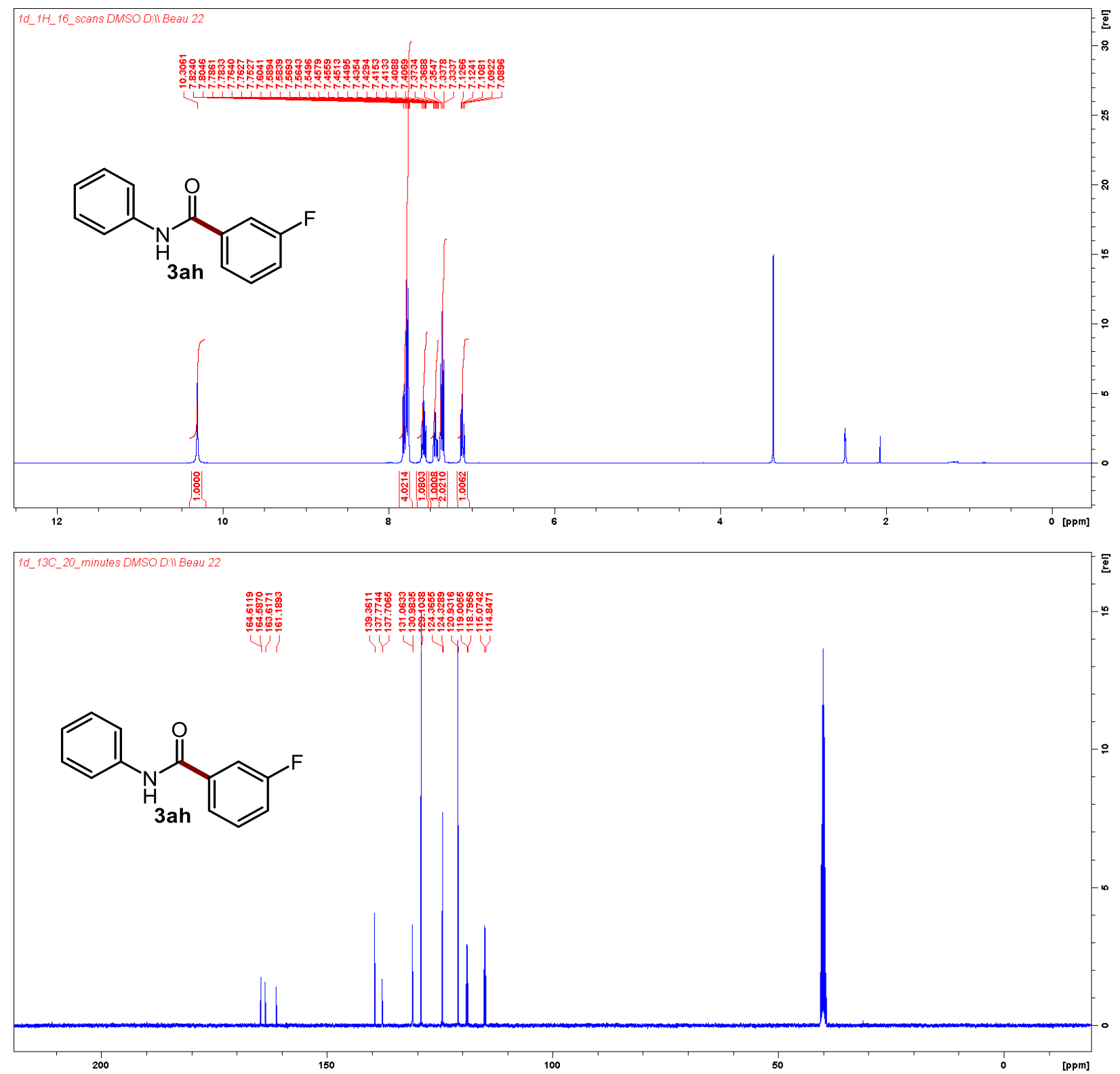

Proton: JD-10-13-1 Carbon: JD-10-13-1 

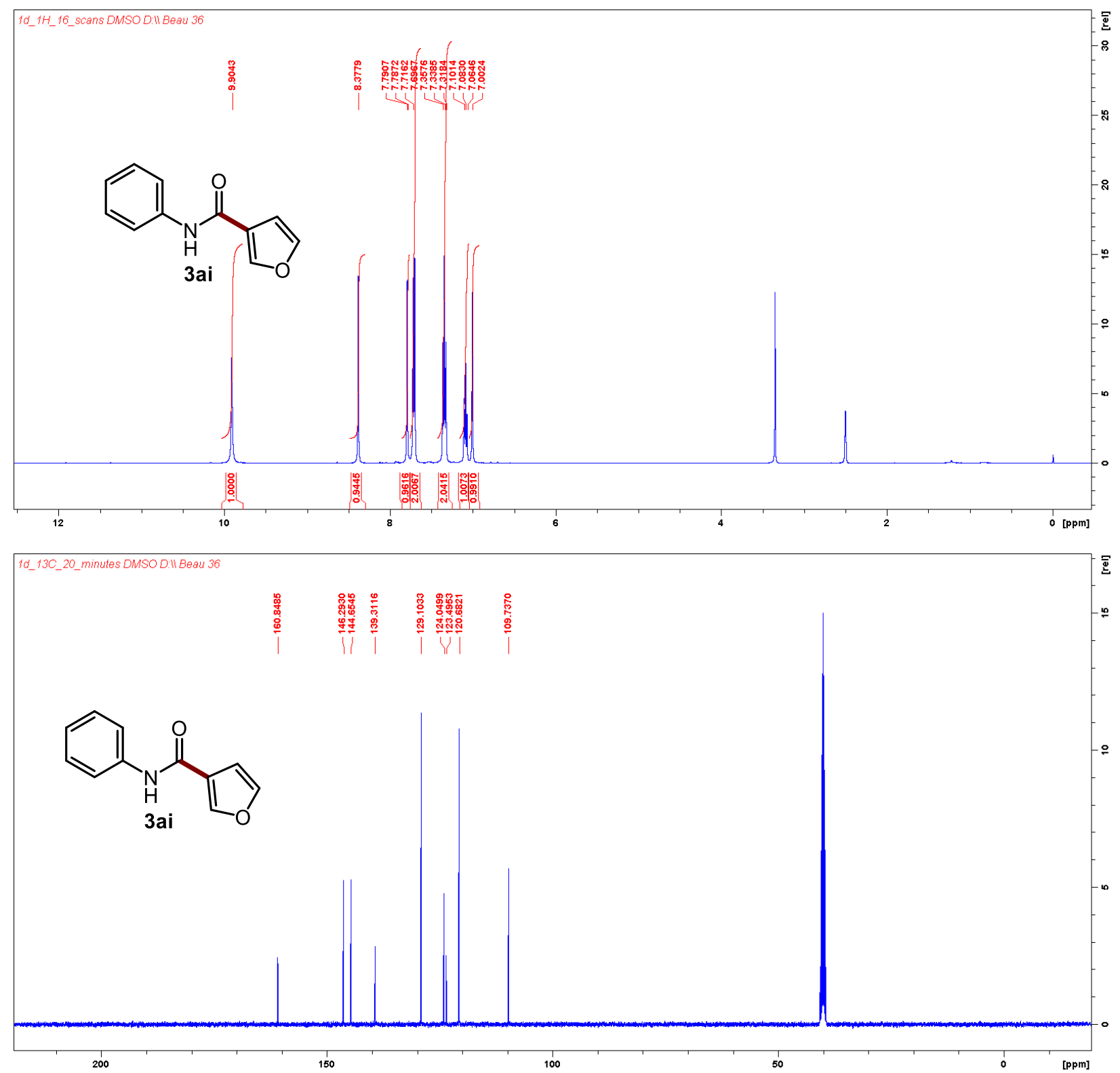

Proton: JD-10-43-5 Carbon: JD-10-45-5 

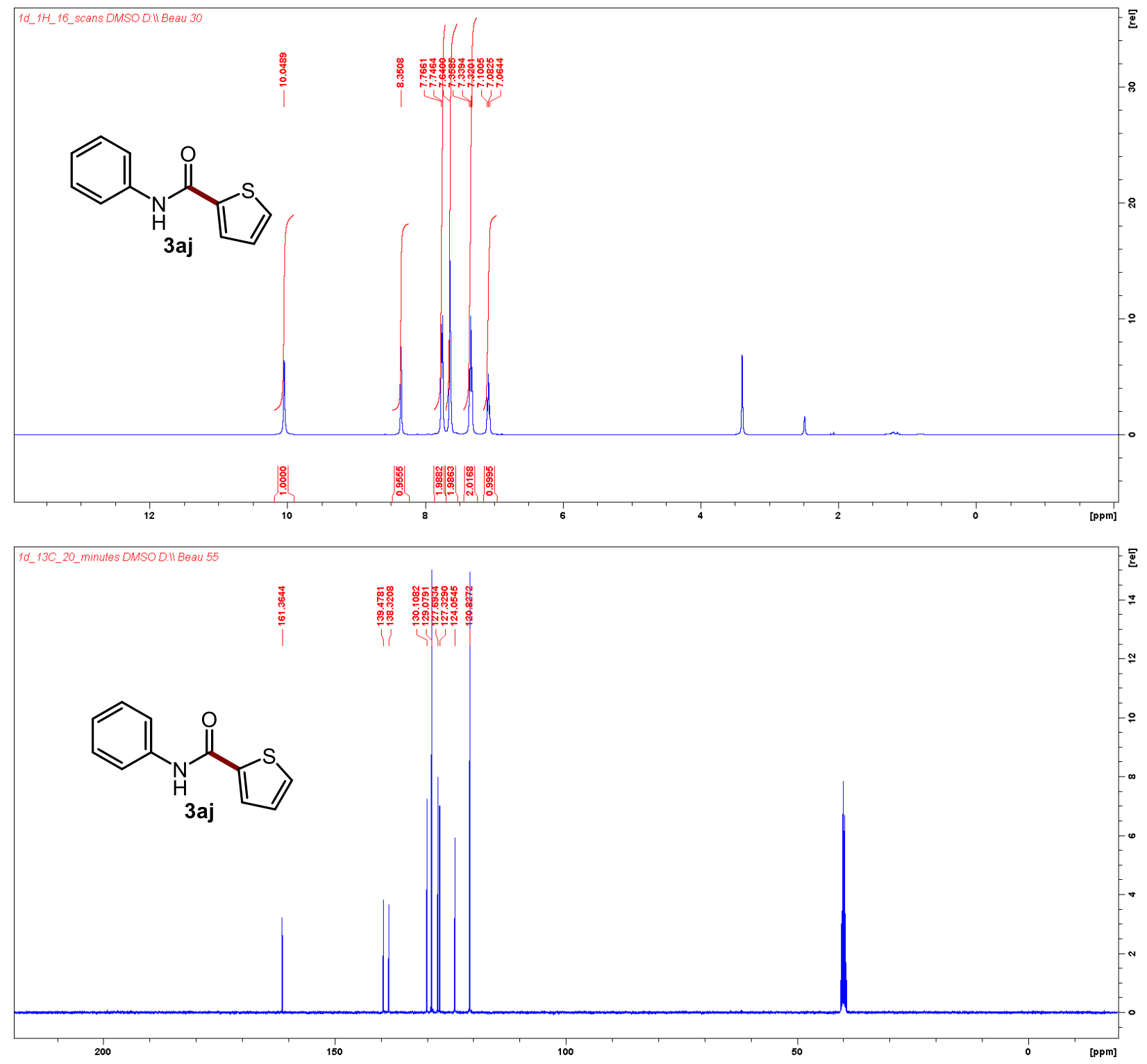

Proton: JD-10-14-2 Carbon: JD-10-14-3 

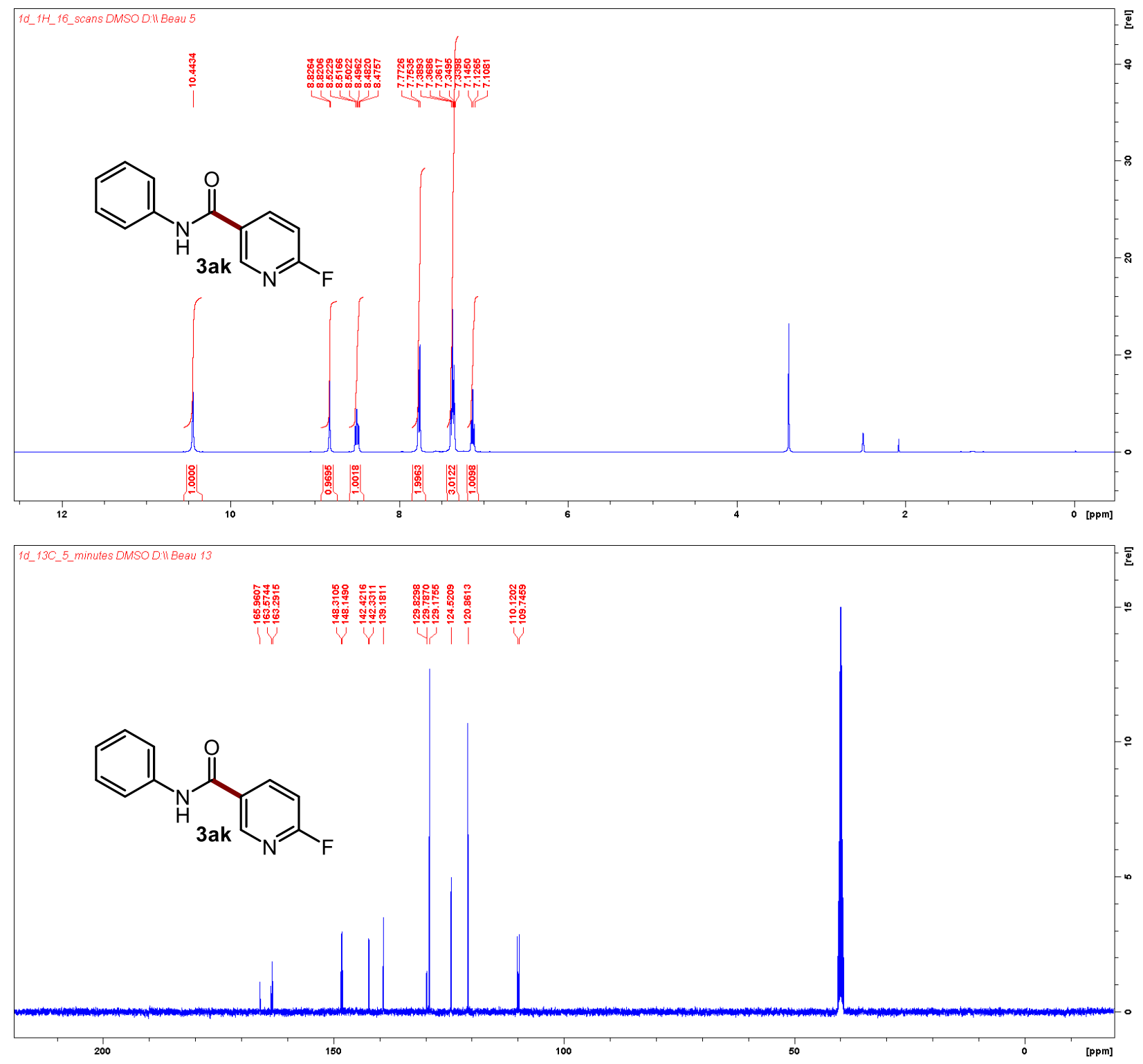

Proton: JD-11-43-1B Carbon: JD-11-43-1B 


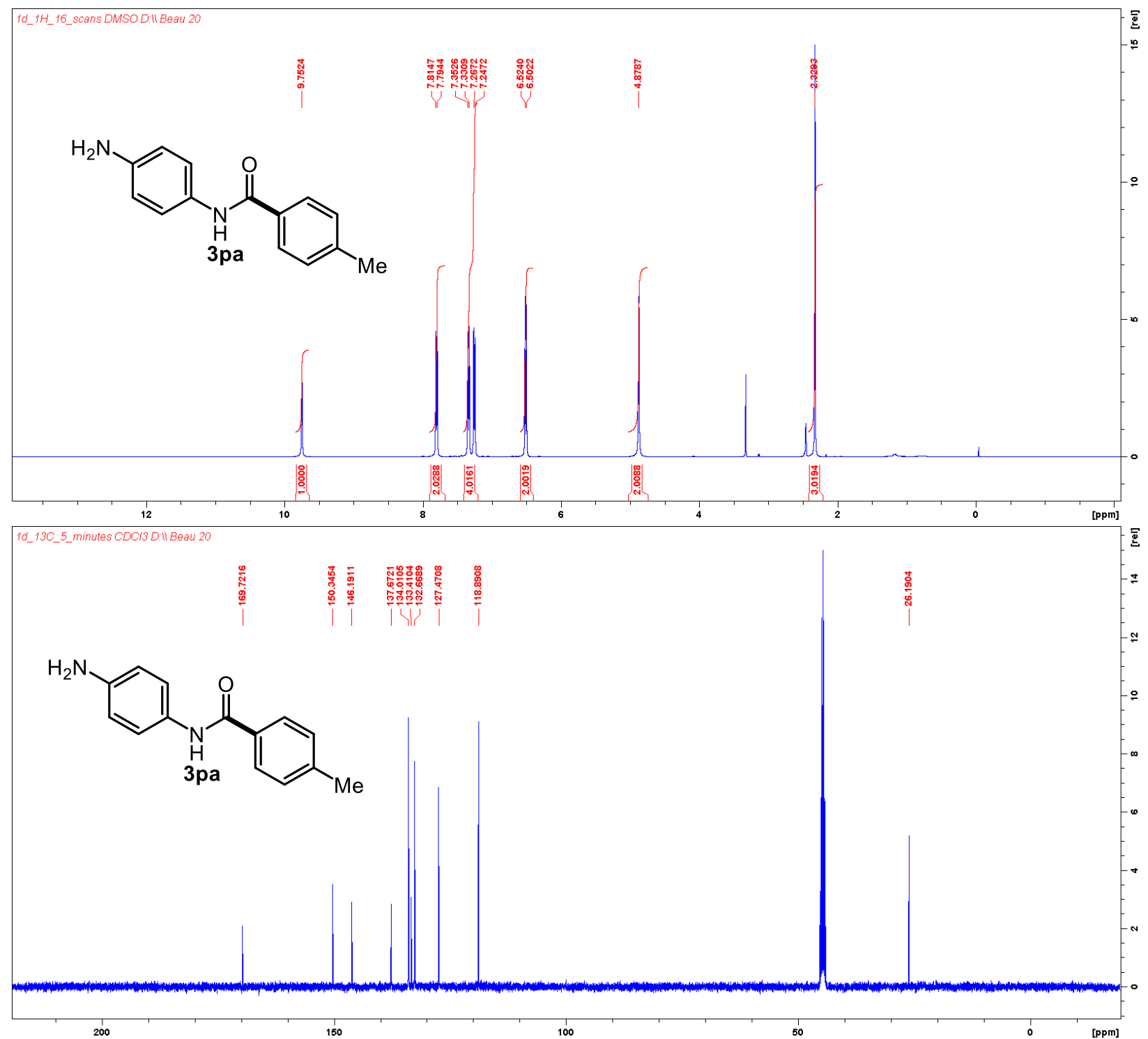

Proton: JD-10-24-3 

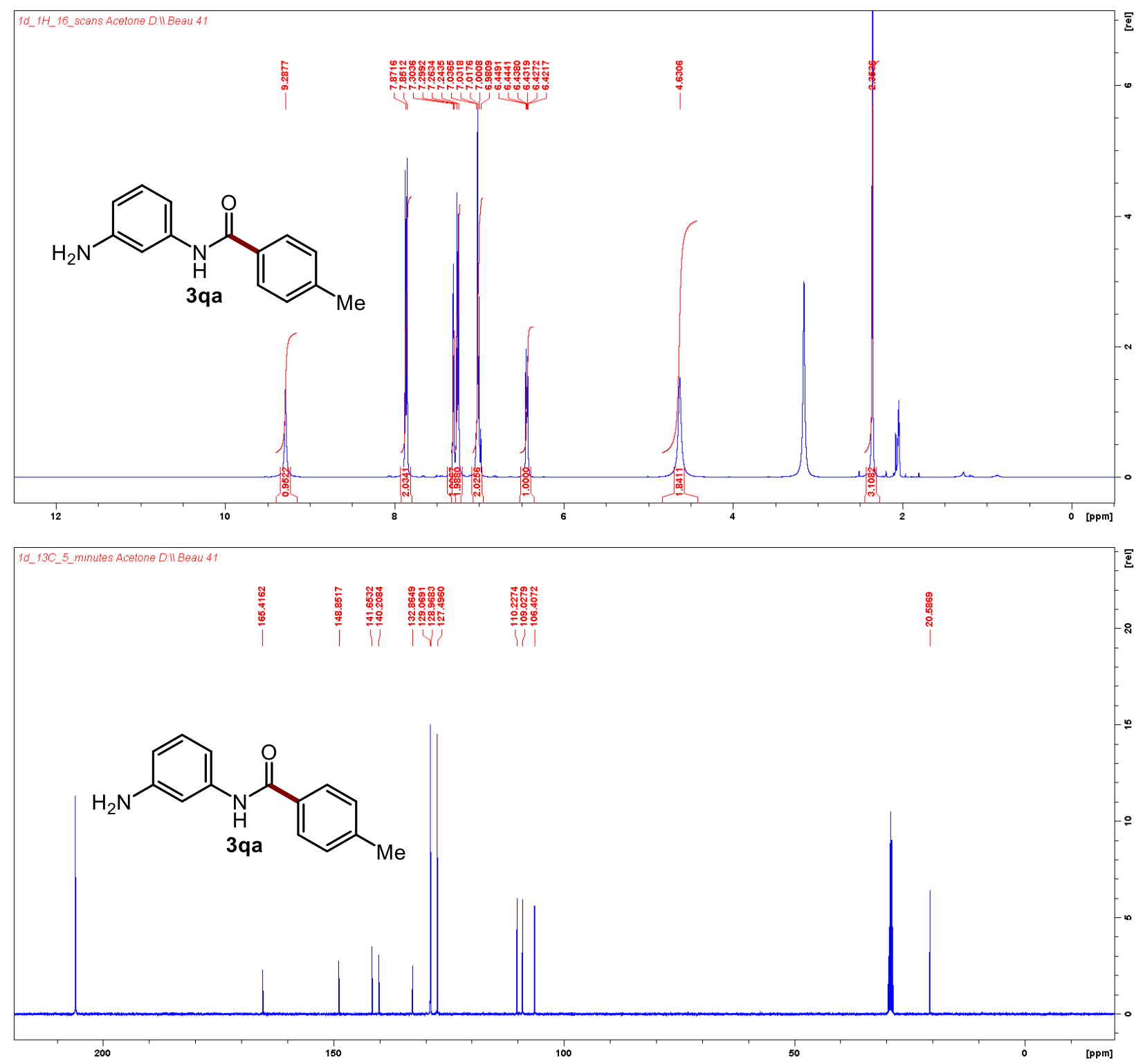

Proton: JD-10-66-2B Carbon: JD-10-66-2B 

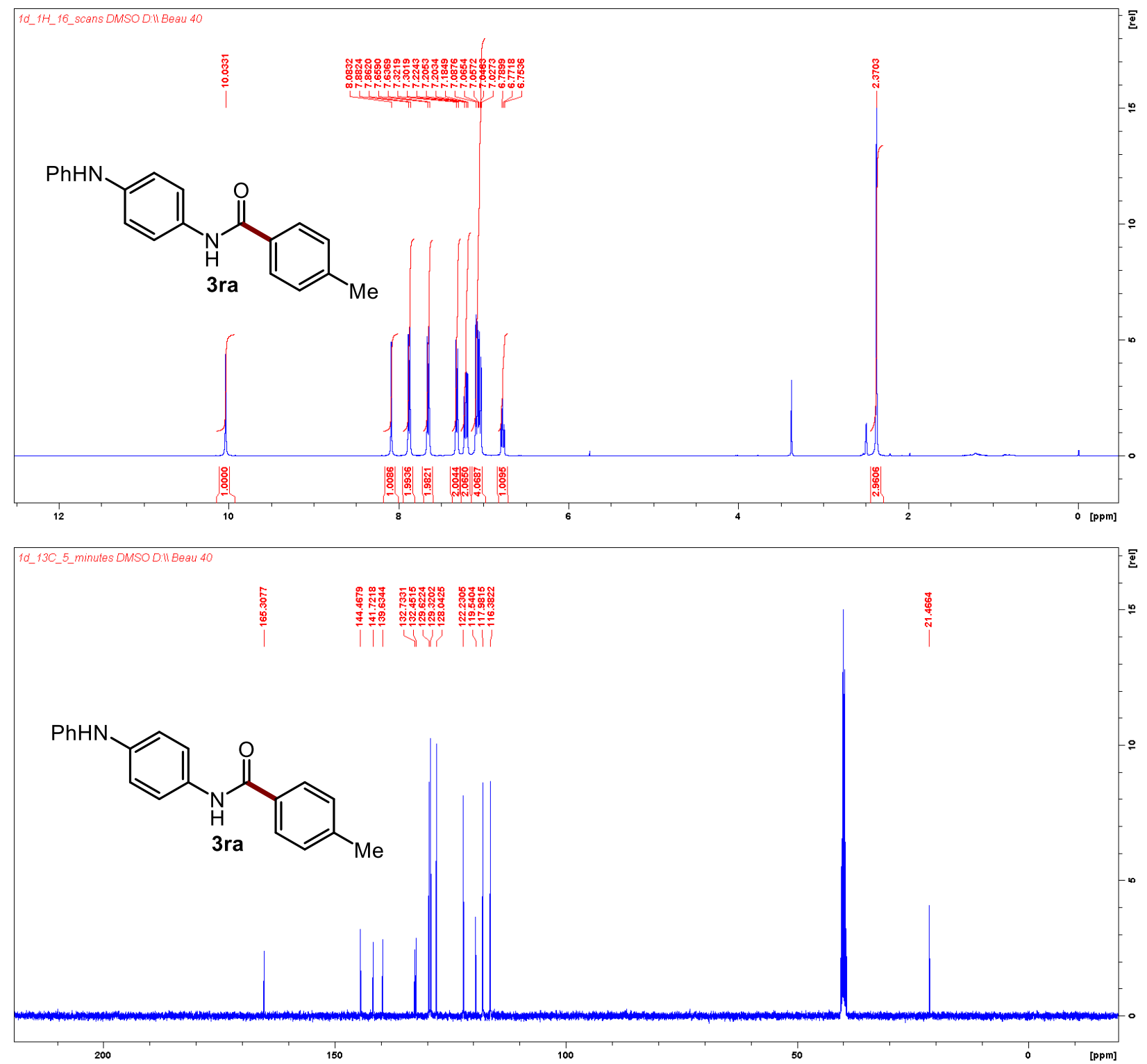

Proton: JD-11-14-2A Carbon: JD-11-14-2A 

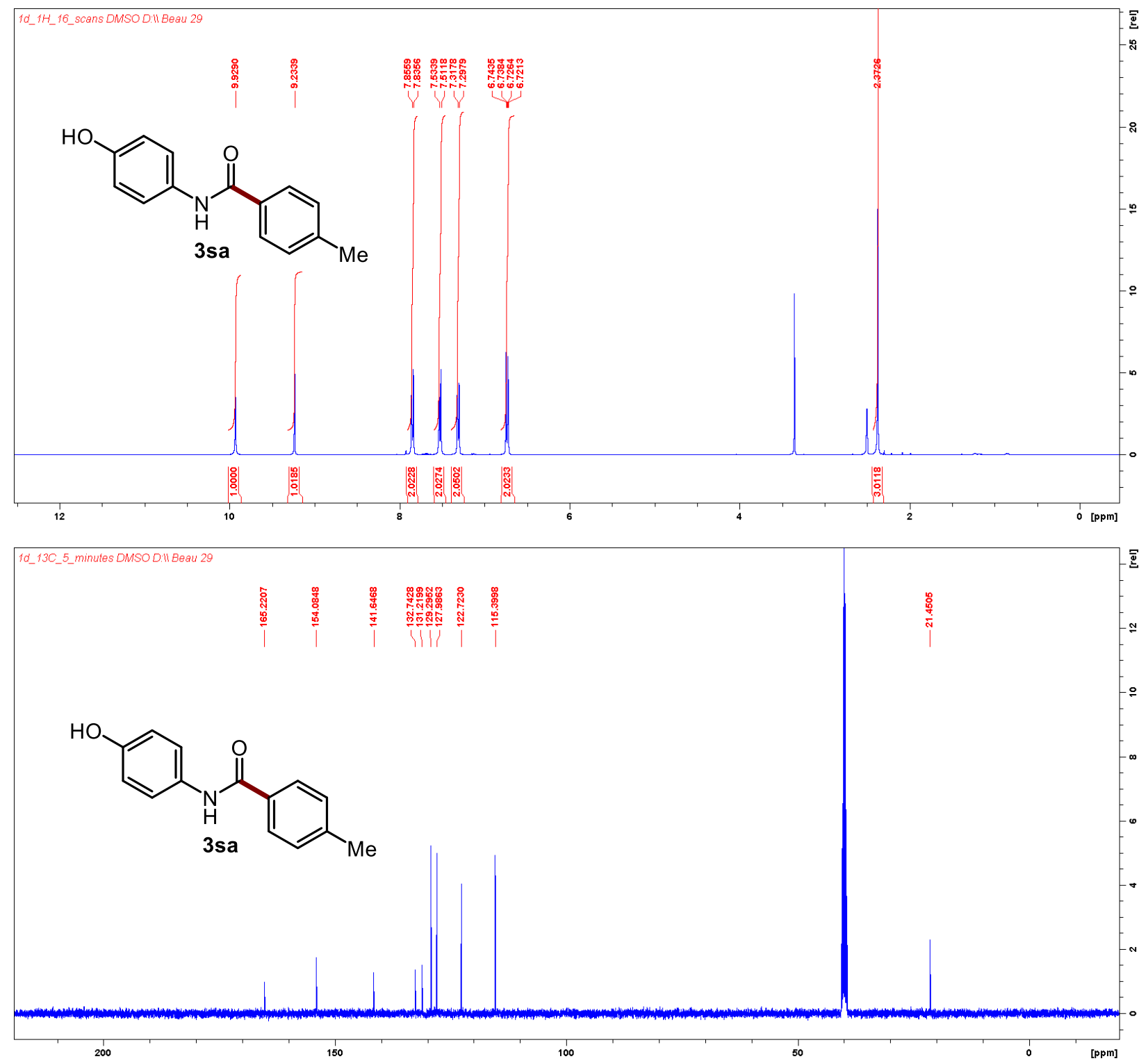

Proton: JD-12-Phenol-characterization 

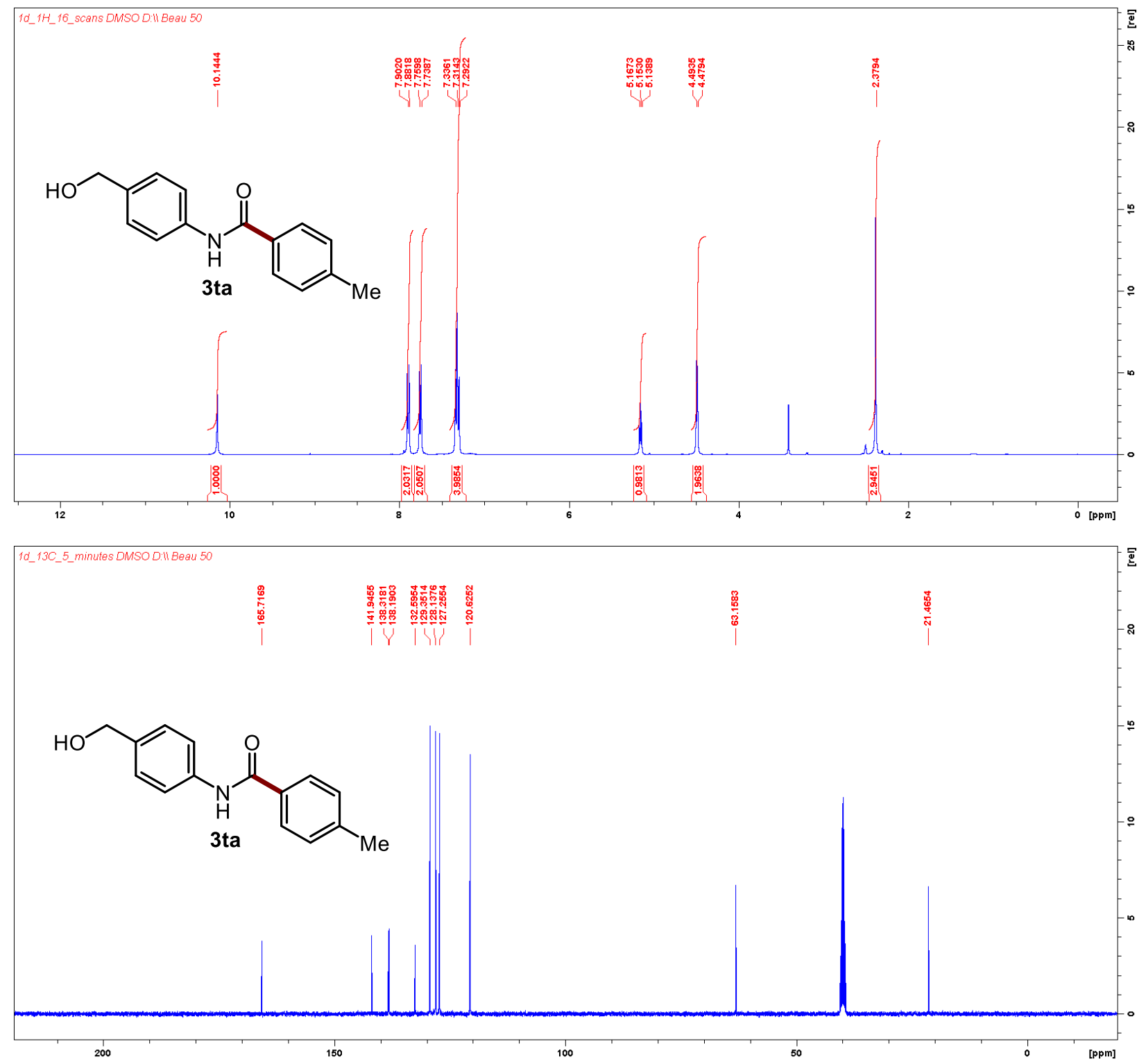

Proton: JD-10-58-1 Carbon: JD-10-58-1 

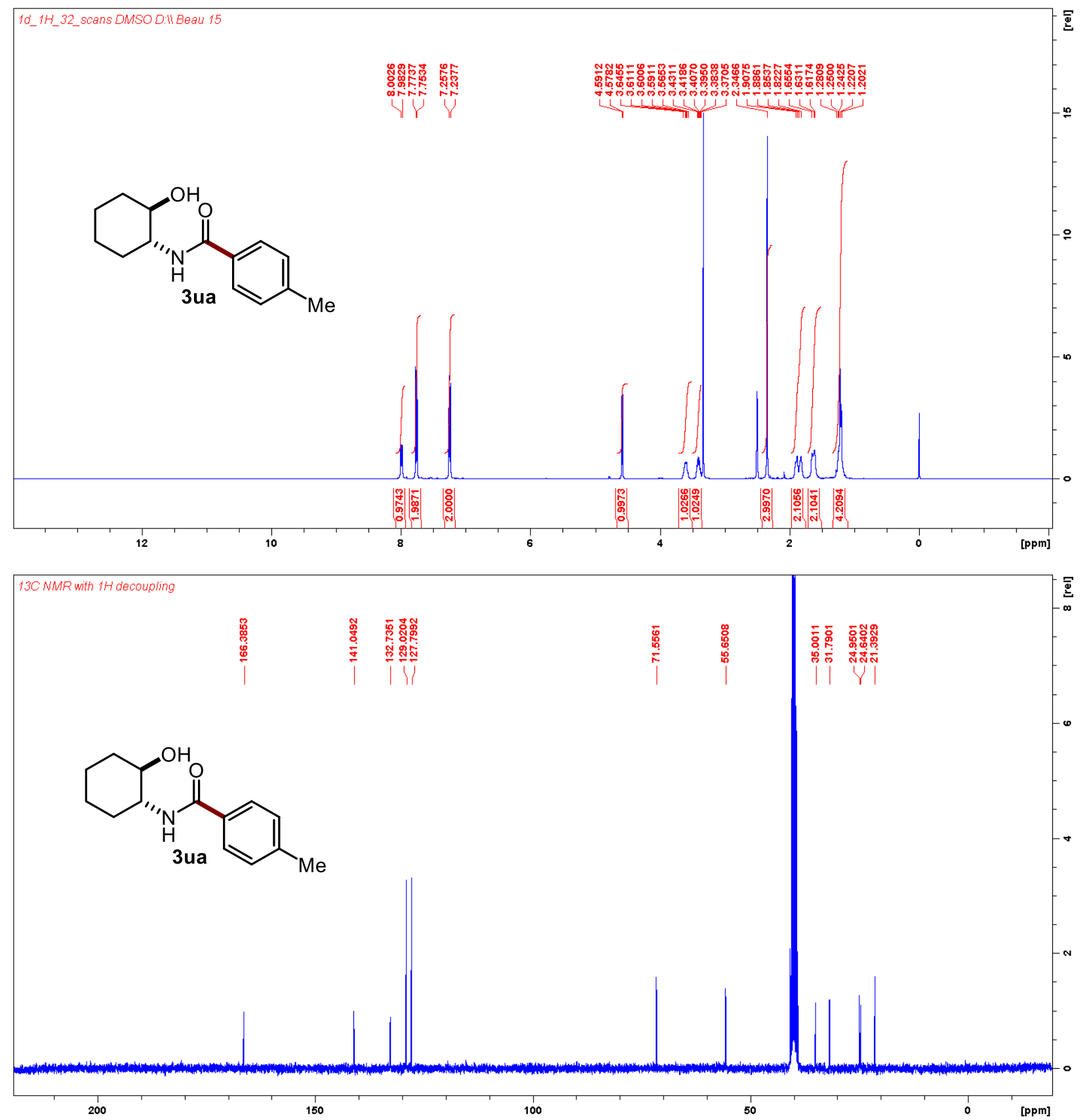

Proton: JD-10-32-2 Carbon: JD-10-32-3 

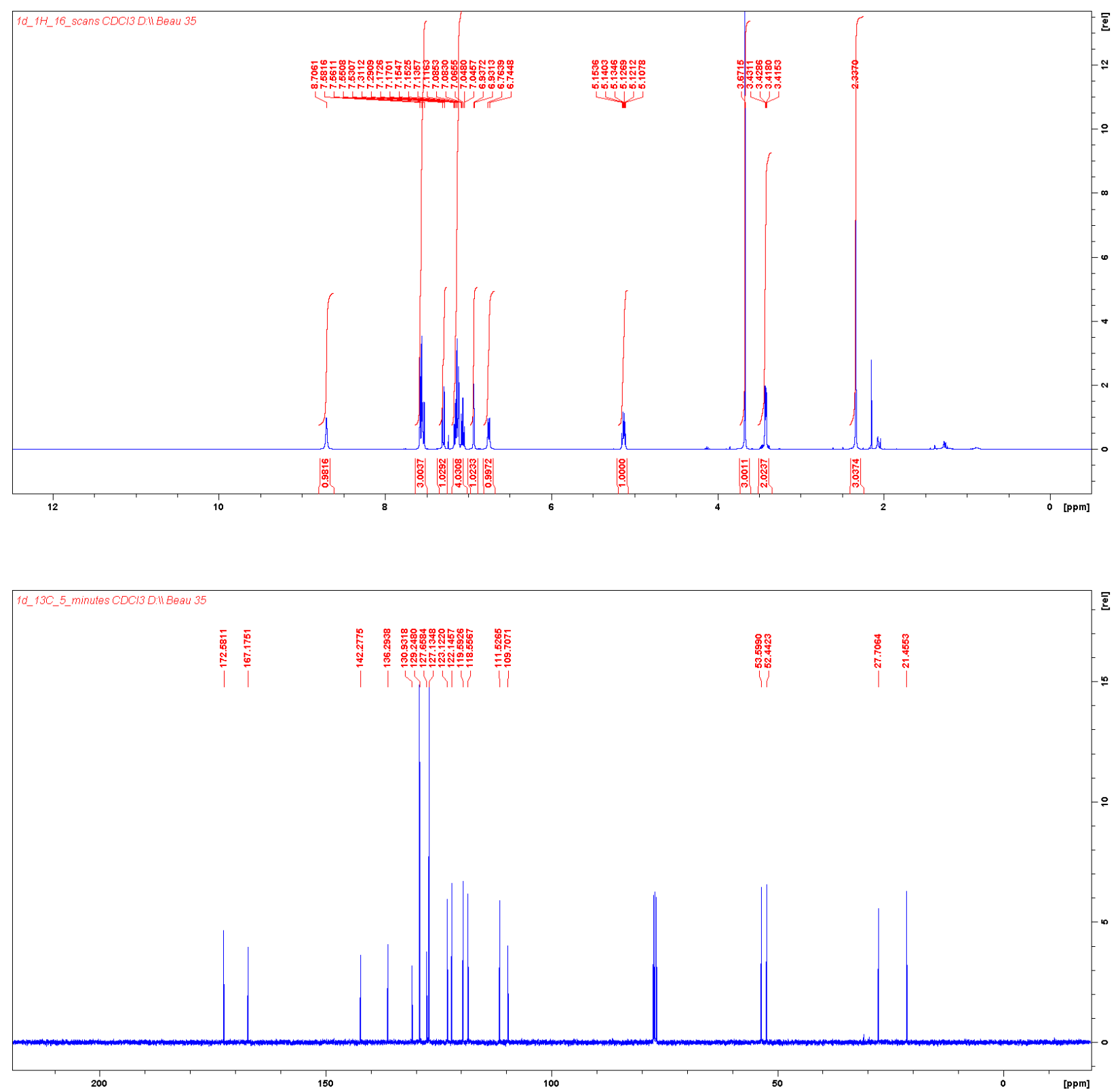

Proton: JD-13-2-5A Carbon: JD-13-2-5A 

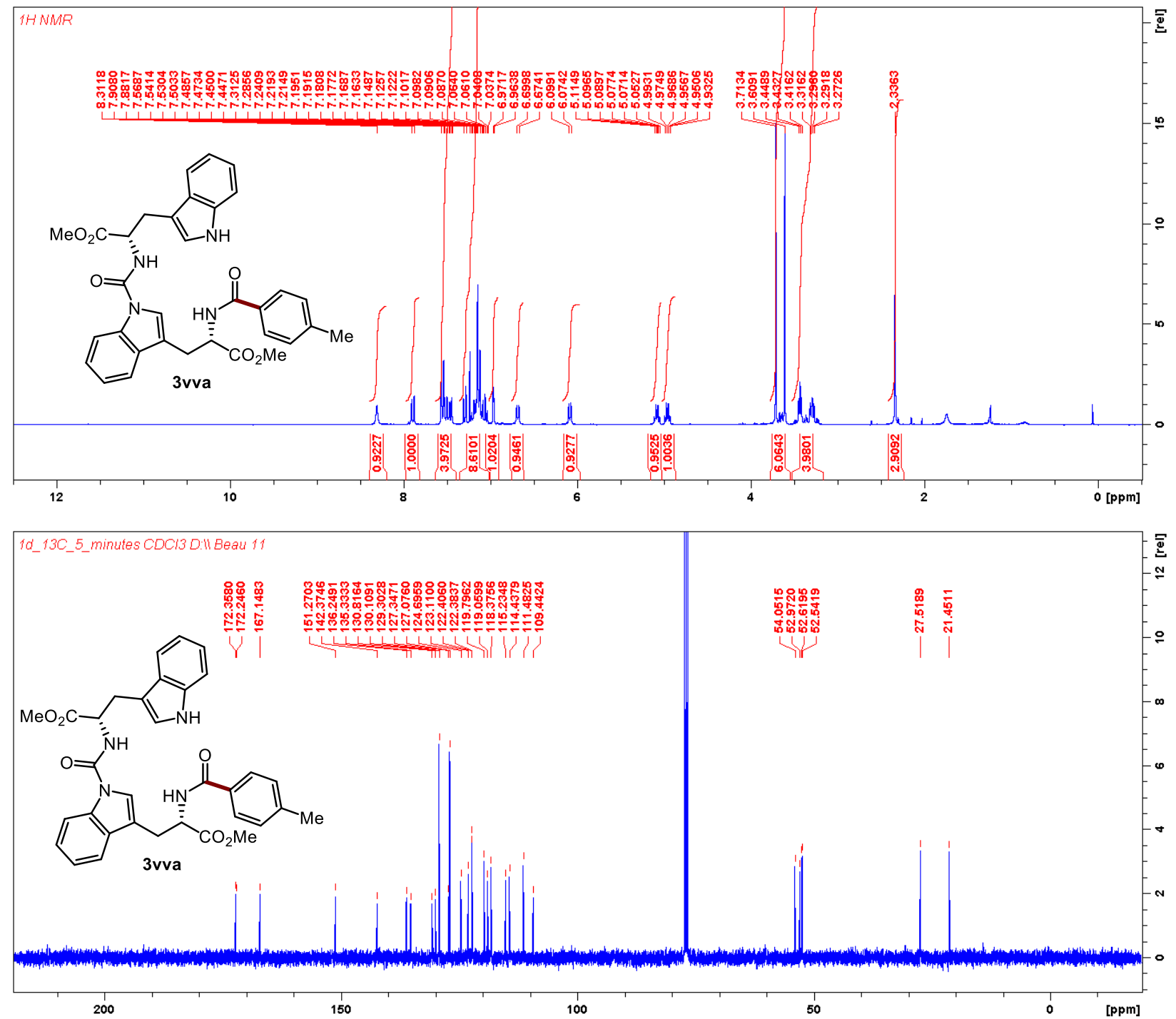

Proton: JD-13-6-2A Carbon: JD-13-5-2B 

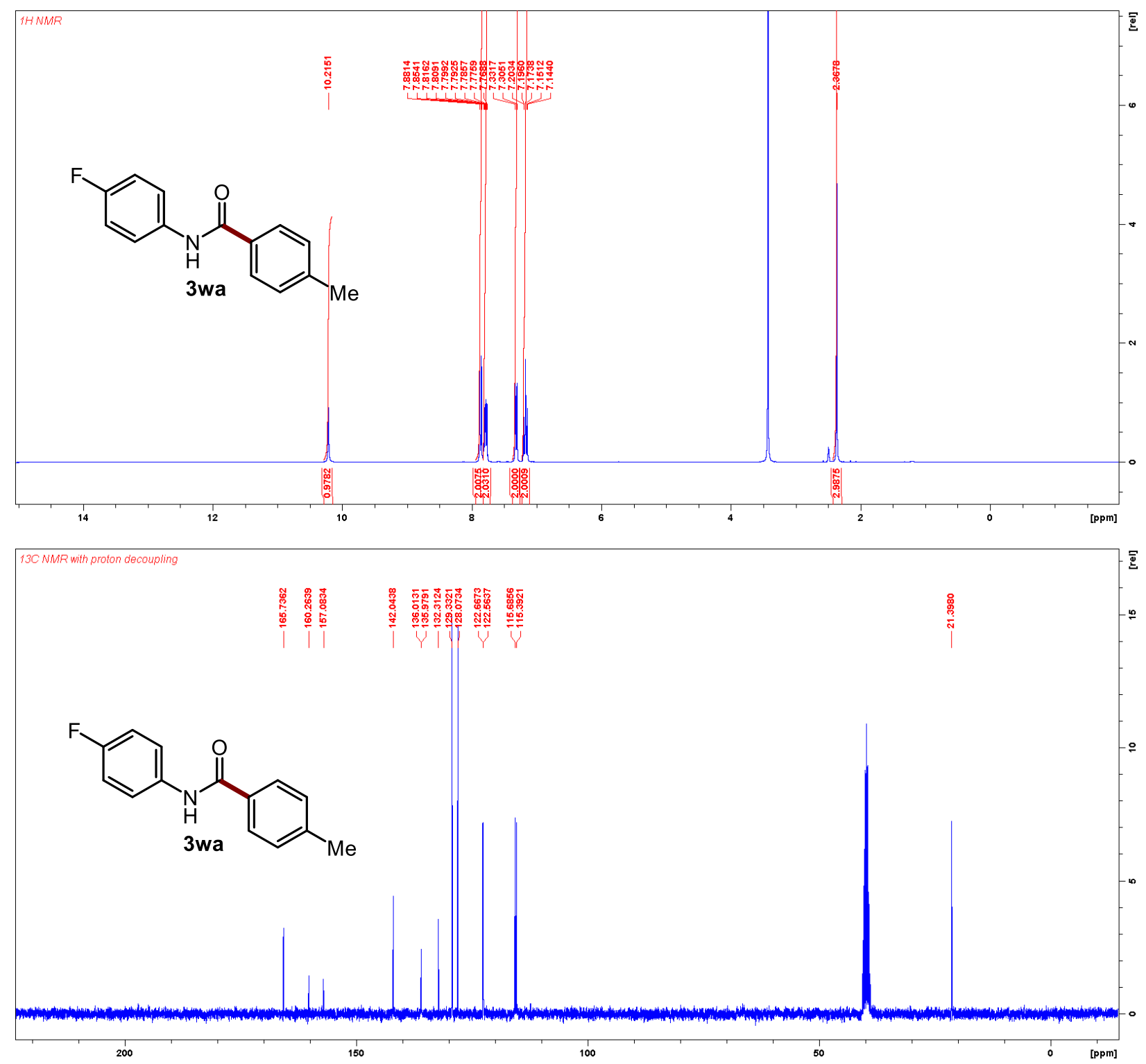

Proton: JD-13-6-2 Carbon: JD-13-6-2 


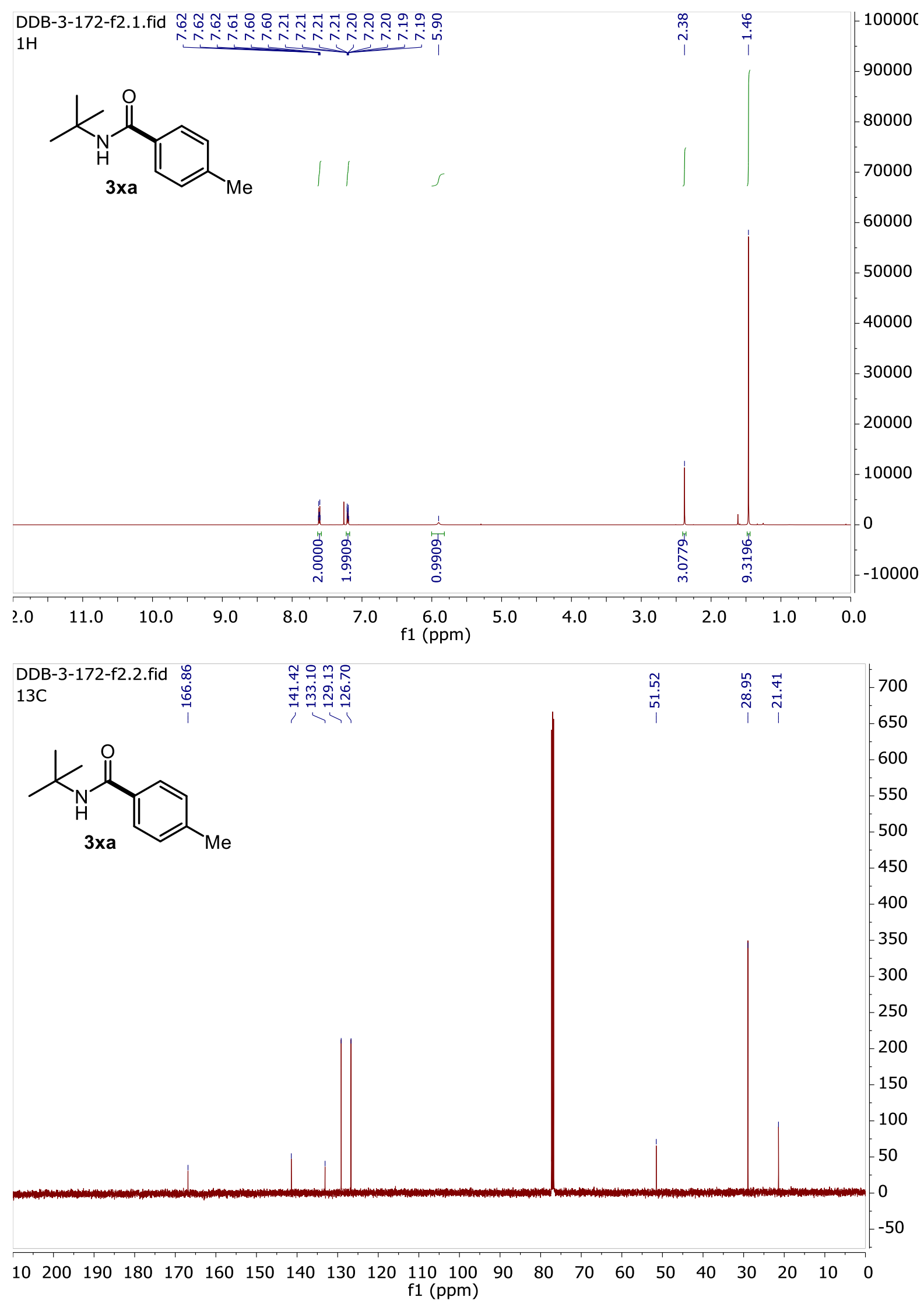

